\title{
Computation and Modelling of Convection Heat Transfer of Supercritical Fluids
}

\author{
by
}

\section{Chukwudi Azih}

\begin{abstract}
A thesis submitted to the Faculty of Graduate and Postdoctoral Affairs in partial fulfillment of the requirements for the degree of

Doctor of Philosophy

in

Mechanical Engineering
\end{abstract}

Carleton University

Ottawa, Ontario

(C) 2018

Chukwudi Azih 
Dedicated to my family 


\section{Abstract}

Current literature suggests that large spatial gradients of thermophysical properties, which occur in the vicinity of the pseudo-critical thermodynamic state, may result in significant variations in forced-convection heat transfer rates. Specifically, these property gradients induce inertia- and buoyancy-driven flow phenomena that may enhance or deteriorate the turbulence-dominated heat convection process. Understanding of these inertia/buoyancy-driven mechanisms has not been sufficiently established to date. Consequently, the full set of dynamic similarity parameters remains to be identified. Through direct numerical simulations of turbulent boundary layers and channel flows, the present study investigates the characteristics of the flow structures of turbulence in heated flows of supercritical water under buoyant and non-buoyant conditions.

In the absence of buoyancy forces, notable reductions in the density and viscosity in close proximity of the heated wall are observed to promote an increase in the wall shear stress, with resultant loss of coherence of the new near-wall flow structures. This leads to the dominance of larger-scale structures in the wall-normal thermal mixing process that comes at the expense of the smaller-scale thermal mixing, and yields a net reduction in the overall thermal mixing.

Under the influence of wall-normal gravitational acceleration, the wall-normal density gradients are noted to enhance ejection motions due to baroclinic vorticity generation on the lower wall of the channel, thus providing additional wall-normal thermal mixing. 
Along the upper wall, the same mechanism generates streamwise vorticity of the opposing sense, causing a net reduction in thermal mixing. In the case of downstreamoriented gravitational acceleration, baroclinic vorticity generation driven by spanwise density gradients causes additional wall-normal thermal mixing by promoting largerscale ejection and sweep motions.

Based on the results of the foregoing direct numerical simulations and complemented by relevant information in the published literature, dynamic similarity criteria for heated flows of supercritical fluids are proposed. Particular consideration is given to the influence of spatial gradients in density in the form of baroclinic forces on such flows. The proposed similarity criteria are successfully validated using data from multiple working fluids, and are used as a basis for a new criterion for the onset of deteriorated heat transfer. 


\section{Preface}

This thesis is produced in an integrated, article-based, format as defined in Section 12.4 of the 2017-2018 General Regulations of the Carleton University Department of Graduate Studies. It consists of the following journal articles that are published.

1. Azih, C. Brinkerhoff, J.R., \& Yaras, M.I., 2012, 'Direct numerical simulation of convective heat transfer in a zero-pressure-gradient boundary-layer with supercritical water', Journal of Thermal Science, 21, pp.49-59.

This article received a best paper designation at the 10th International Symposium on Experimental Computational Aerothermodynamics of Internal Flows in Brussels, Belgium.

2. Azih, C. \& Yaras, M.I., 2018, 'Effects of spatial gradients in thermophysical properties on the topology of turbulence in heated channel flow of supercritical fluids', Physics of Fluids, 30(1), p.015108, 23 pages.

This article was declared by the journal editors to be one of the top articles in Physics of Fluids, and was accordingly designated as a featured article.

3. Azih, C. \& Yaras, M.I., 2017, 'Similarity criteria for modelling mixed-convection heat transfer in ducted flows of supercritical fluids', Journal of Heat Transfer, 139(12), p.122501, 13 pages.

The first chapter of the thesis provides a brief technical background targeted to nonexpert readers in the fields of turbulent flows and related heat transfer mechanisms. The 
second chapter provides an introduction to the thesis manuscript. Chapter 3 outlines the thesis objectives. Chapters 4,5 , and 6 are respective reprints of the articles enumerated above and are followed by Chapter 7, which summarizes the contributions of the individual papers to addressing the objectives of the thesis.

The first and third papers that comprise this thesis are copyrighted material and permissions to reprint these documents are provided in Appendix D. The second article is an open access article for which broad publishing and distribution rights remain with the American Institute of Physics (AIP). Readers who wish to cite sections of this thesis are encouraged to cite the papers.

For consistency of format and terminology throughout the thesis manuscript, several modifications have been made to the journal articles integrated into the manuscript. The papers have been re-set from their respective journal-specific formats to the thesis format. The conclusion and reference sections from each of the journal articles have been amalgamated into unified conclusion and reference sections of the thesis manuscript, and citations have been reformatted to match the Harvard citation style. A unified nomenclature section is provided and symbols in individual articles are modified to suit. The labels of the test cases in the first journal article have been changed to be consistent with that of the second article. The Nusselt number correlation in the first article has also been changed to be consistent with the more relevant version used in a later work of the author's research group (Dave et al., 2013). The last section in the first article, which 
provides hypotheses on boundary layer instabilities in the flow of supercritical fluids, is not included as it has been more thoroughly discussed in the second article.

The author of this thesis, Chukwudi Azih, was principally involved in conceptualization, conducting the analyses, interpreting the data, and preparing material for the listed journal articles. The co-authors in the above-enumerated articles served in supervisory capacities for the research performed in the respective articles. Professor Metin I. Yaras was involved in conceptualization of the methodology for the research. He also provided technical guidance and supervision of the research, and reviewed the manuscripts. In the first article, Joshua R. Brinkerhoff was involved in supervising the numerical set-up and execution of the simulations as well as reviewing the manuscript. 


\section{Acknowledgements}

The work in this thesis was facilitated by financial support through the Canada Gen-IV National Program funded by Natural Resources Canada, Atomic Energy of Canada Limited, and Natural Sciences and Engineering Research Council of Canada.

Professor Yaras' uncompromising diligence has helped me reach a technical potential that I did not envision I could attain as pertains to undertaking fundamental and applied research in the field of science. I gratefully acknowledge the support, advice, and constructive feedback that he has provided to me throughout the course of the present endeavour. I acknowledge the major role professor Junjie Gu played in convincing me to pursue post-graduate studies. I am grateful to Carleton University for providing leadership, teaching, human resources, and project management opportunities all of which are essential to be an effective researcher in the field of science.

I gratefully acknowledge the fellow members of Professor Yaras' research group: Joshua R. Brinkerhoff, Masih Balouch, Shawn Reinink, Nikunj Dave, Aneesh John, Majed Piedra Abu Sharrar, Hristo Valtchanov, Mustafa Mogri, Mustafa Siddiqui, Andrew Copping, and many others. We shared knowledge, insights, tips, jokes, and frustrations as we studied and researched together. I would like to thank Neil McFadyen and Bruce Johnston for their technical assistance in maintaining the resources required to perform the computations to execute this thesis. The administrative help of Nancy Powell, Christie Egbert, Irene Helder, and the late Marlene Groves are greatly appreciated. 
There are many other elements that enabled me to endure this research endeavour. My faith in God, encouragement from my church community, support and encouragement from my colleagues at Canadian Nuclear Laboratories, and occasional but much needed distractions from my friends. Most of all, I am indebted to my family, My wife Kayla, my children Mira and Zeke, my siblings Ekene and Anuli, my parents Godwin and Chioma, and my in-laws Gary, Faye, and Kevin. I am gratefully for their patience, love, understanding, motivation, support, and encouragement as I went through this Journey. 


\section{Table of Contents}

Abstract.............................................................................................................................

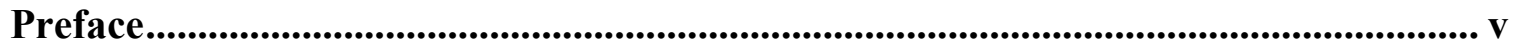

Acknowledgements ....................................................................................................... viii

Table of Contents ........................................................................................................................... $\mathrm{x}$

List of Tables ............................................................................................................................. Xv

List of Figures............................................................................................................................. xvi

Nomenclature .................................................................................................................... xxvi

1 Chapter: Outline and Background .............................................................................. 1

$1.1 \quad$ The Supercritical Thermodynamic State ….............................................................. 2

1.2 Background on Fluid Mechanics and Heat Transfer ................................................. 3

1.2.1 Fluid Flows within Wall-Bounded Geometries ..................................................... 4

1.2.2 Forced Convection Heat Transfer in Wall-Bounded Flows ........................................5

1.3 Physical Mechanisms that Drive Turbulent Flows and Heat Transfer ......................... 7

1.3.1 Laminar-to-Turbulent Flow Transition in Unheated Flows .................................... 8

1.3.2 Effect of Heat Transfer on Laminar-to-Turbulent Flow Transition .......................... 10

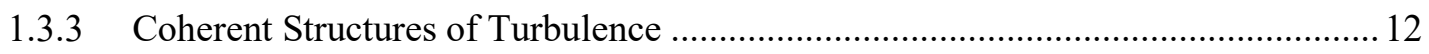

1.4 Computational Methods for Fluid Mechanics and Heat Transfer ..............................20

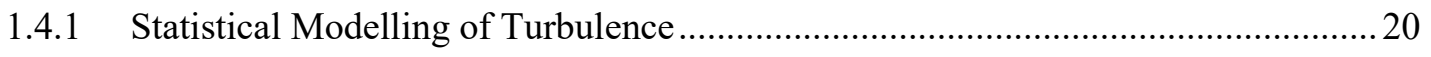

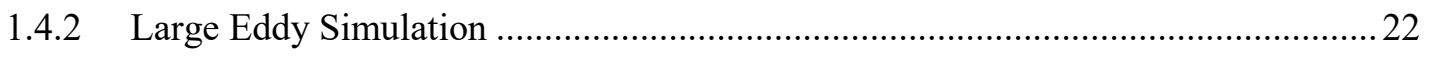

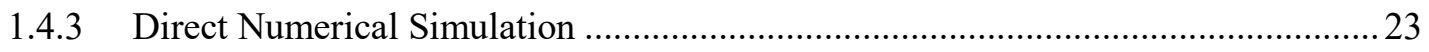

2 Chapter: Introduction and Literature Review …….................................................. 25

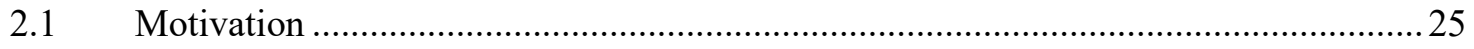




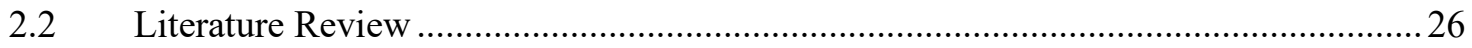

2.2.1 Forced Convection Heat Transfer to Flows of Supercritical Fluids........................26

2.2.2 Statistical Turbulence Modelling of Flows of Supercritical Fluids ......................... 31

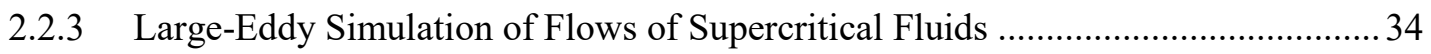

2.2.4 Direct Numerical Simulation of Flows of Supercritical Fluids.................................36

2.2.5 Summary of Literature Review on Heat Transfer in Supercritical Fluids................. 38

3 Chapter: Thesis Objectives ............................................................................................... 44

4 Chapter: Direct Numerical Simulation of Convective Heat Transfer in a ZeroPressure-Gradient Boundary Layer with Supercritical Water ............................... 45

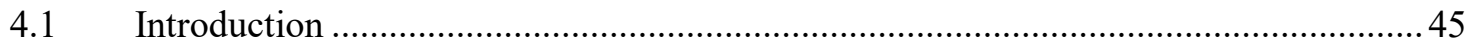

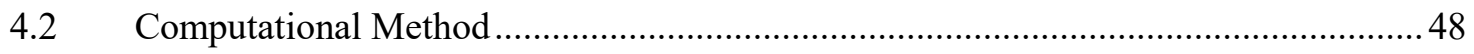

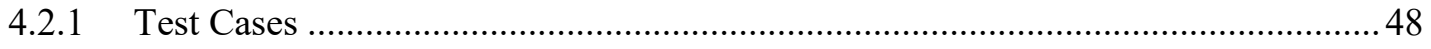

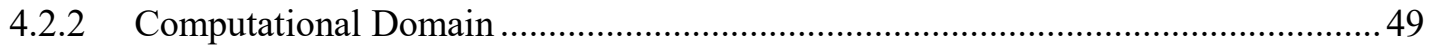

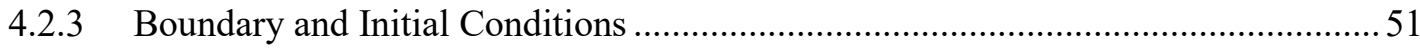

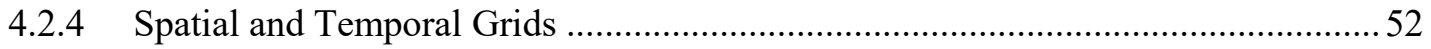

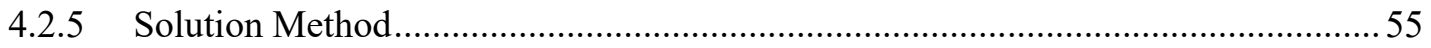

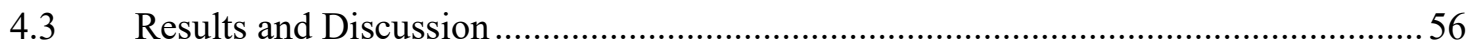

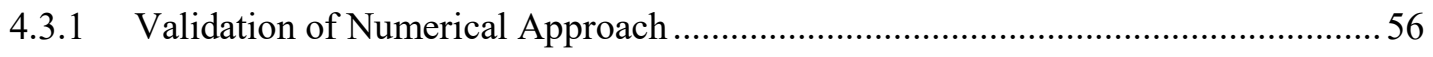

4.3.2 Effect of Supercritical Thermodynamic State on Heat Transfer .............................60

5 Chapter: Effects of spatial gradients in thermophysical properties on the topology of turbulence in heated channel flow of supercritical fluids ........................ 65

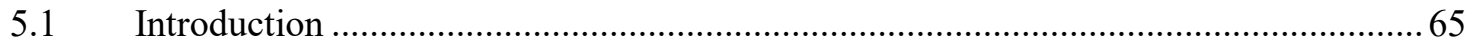

5.1.1 Turbulence in Single-Phase Subcritical Wall-Bounded Flows ................................ 67

5.1.2 Turbulence in Heated Flows of Supercritical Fluids ............................................. 70

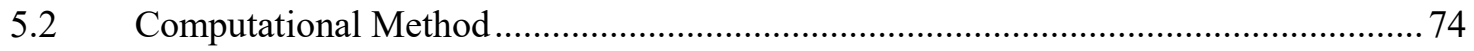




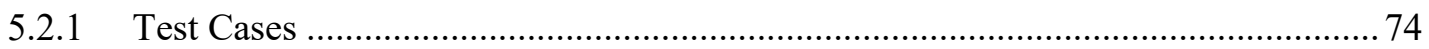

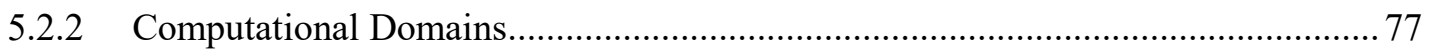

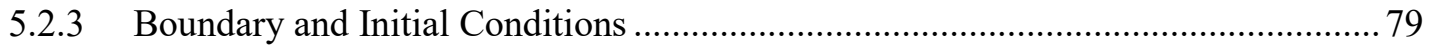

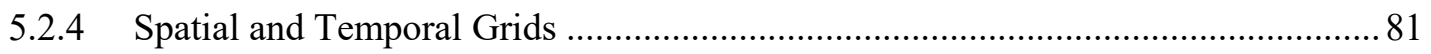

5.2.5 Governing Equation and Solution Method........................................................ 83

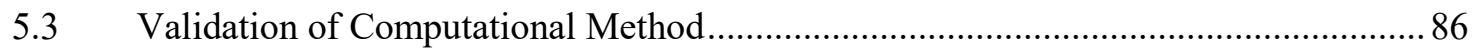

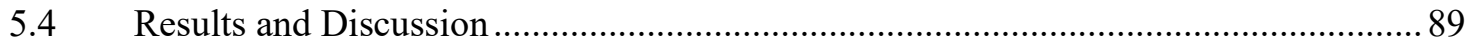

5.4.1 Coherent Flow Structures in the Absence of Spatial Variations in Thermophysical

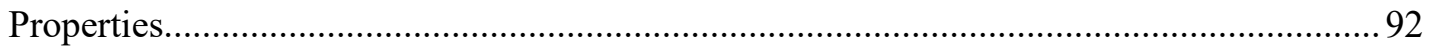

5.4.2 Effects of Spatial Gradients in Thermophysical Properties on the Coherent Flow Structures of Turbulence in the Absence of Buoyancy Forces .......................................... 96

5.4.2.1 Flow Development near the Inflow Plane _..................................................... 98

5.4.2.2 Interaction between Near-Wall Wavepackets ............................................... 104

5.4.2.3 Regeneration of Organized Near-Wall Coherent Structures of Turbulence.. 106

5.4.2.4 Deterioration and Enhancement of Heat Transfer ........................................ 110

5.4.3 Effect of Spatial Gradients in Thermophysical Properties on the Coherent Structures of Turbulence in the Presence of Buoyancy Forces ....................................................... 112

5.4.3.1 Flow Development along the Lower Wall of the Case with Wall-Normal

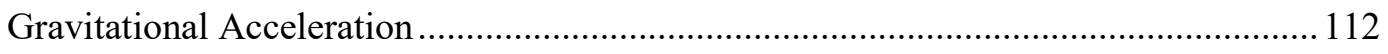

5.4.3.2 Flow Development along the Upper Wall of the Case with Wall-Normal Gravitational Acceleration 119

5.4.3.3 Flow Development in the Case with Downstream-Oriented Gravitational Acceleration 124

\section{Chapter: Similarity Criteria for Modelling Mixed-Convection Heat transfer in} Ducted Flows of Supercritical Fluids ............................................................................ 129 
6.1 Introduction 129

6.1.1 Dynamic Similarity Criteria for Heat Transfer in Supercritical Fluids 130

6.1.2 State-Space Similarity Criteria for Supercritical Fluids. 134

6.1.3 Validation of Similarity Criteria for Heat Transfer in Supercritical Fluids ..... 135

6.2 Dynamic Similarity Parameters for Heat Transfer in Supercritical Fluids. 138

6.3 Similarity Criteria for the State-Space Distribution of Thermophysical Properties at the Supercritical State. 143

6.4 Similarity Criteria for Mixed-Convection Heat Transfer in Ducted Flows of Supercritical Fluids 150

6.5 Validation of the Similarity Criteria for Heat Transfer in Supercritical Fluids 153

7 Chapter: Conclusion and Recommendations. 165

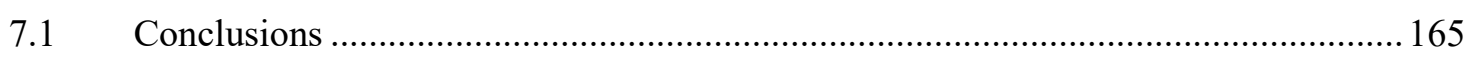

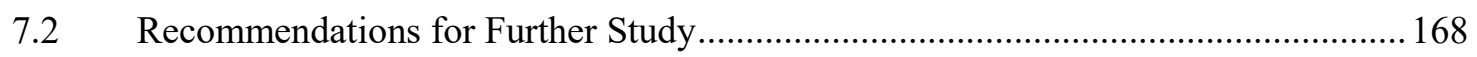

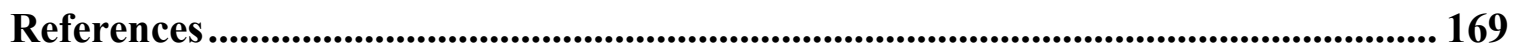

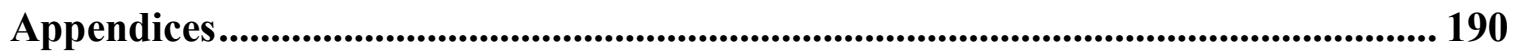

Appendix A Direct Numerical Simulations of Laminar and Turbulent Forced-Convection Heat

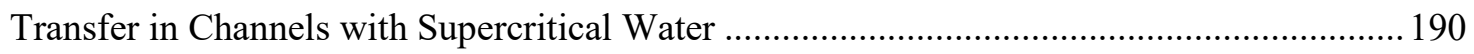

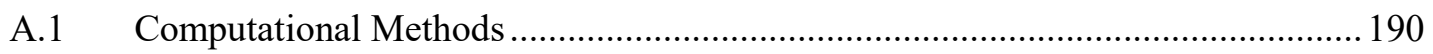

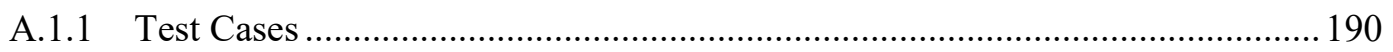

A.1.2 Computational Domains, Boundary Conditions and Initial Conditions ............ 193

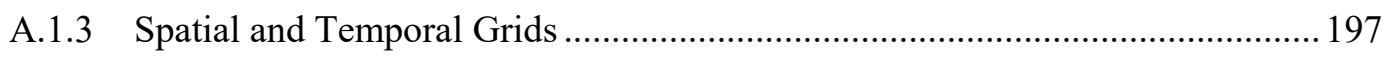

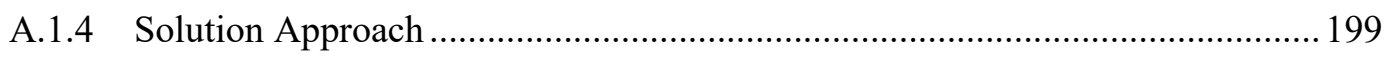

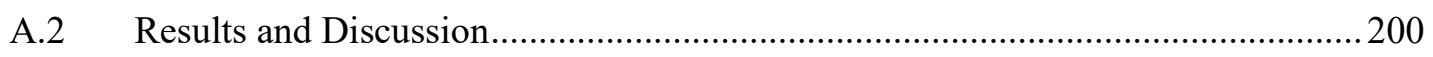

A.2.1 Validation of Computational Method ......................................................... 200

A.2.2 Effect of Wall-Normal Property Variation on Heat Transfer in Laminar Flows202 
Appendix B Numerical and Computational Method ........................................................ 208

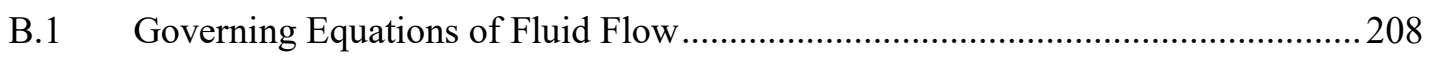

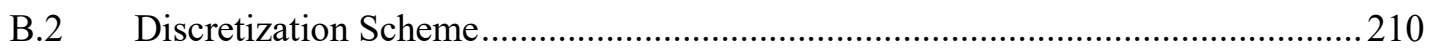

B.2.1 Finite Volume Discretization of Governing Equations....................................211

B.2.2 Order of Accuracy of Discretization Schemes.................................................213

B.2.3 Solution Method for the Linearized System of Equations ................................214

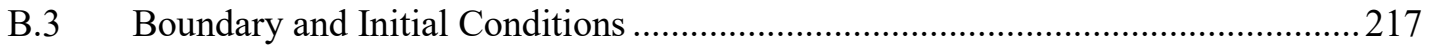

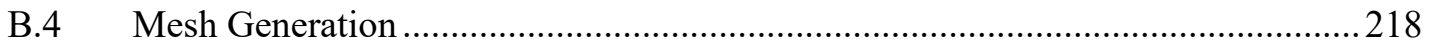

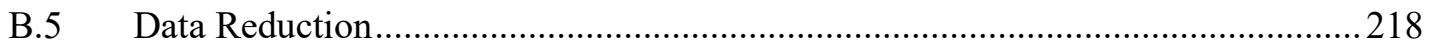

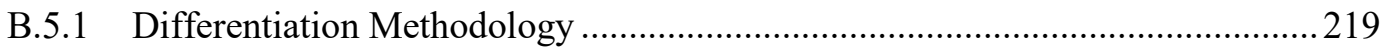

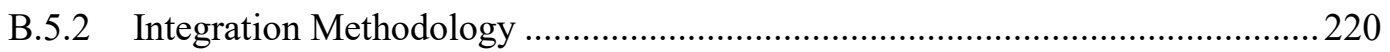

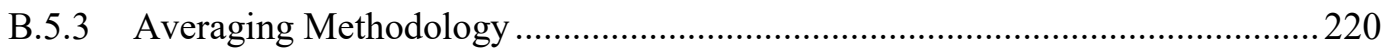

B.5.4 Spectral Analyses Methodology ………....................................................... 221

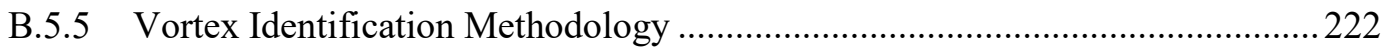

Appendix C Derivation of Non-Dimensional Forms of the Governing Equations and Boundary

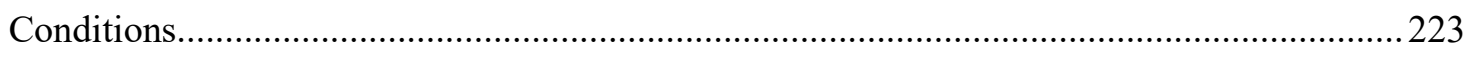

C.1 Derivation of Non-Dimensional Continuity Equation .........................................223

C.2 Derivation of Non-Dimensional Momentum Equation .......................................223

C.3 Derivation of Non-Dimensional Energy Equation ............................................22

C.4 Derivation of Non-Dimensional Heat Transfer Boundary Condition .....................226

Appendix D Permissions to Reproduce Copyrighted Material ..........................................2229 


\section{List of Tables}

Table 2-1: Selected experimental heat transfer studies with supercritical water flowing in tubes. 40

Table 2-2: Selected experimental heat transfer studies with supercritical carbon dioxide flowing in tubes. 41

Table 2-3: Selected experimental heat transfer studies with supercritical helium flowing in tubes. 42

Table 2-4: Selected experimental heat transfer studies with supercritical R134a flowing in tubes. 42

Table 2-5: Selected numerical studies on heat transfer in ducted flows of supercritical fluids. 43

Table 4-1: Test cases for turbulent flow over a flat surface. 48

Table 4-2: Nusselt numbers for the cases with supercritical water as the working fluid. 61 Table 5-1: Simulation parameters for turbulent channel flow cases. 74

Table 5-2: Node spacings corresponding to the maximum value in vicinity of the wall. 82 Table 5-3: Range of wall-normal node spacings for computational domains. 82

Table 5-4: Spanwise wavelength of the dominant coherent flow structures at $y^{+}=15$, $x_{\delta}=9.1-$ most dominant wavelength; $2-2^{\text {nd }}$ most dominant wavelength. 92 Table 6-1: Non-dimensional expressions for wall heat transfer. 134

Table 6-2: The critical pressure and temperature of fluids commonly used in heat transfer studies involving supercritical thermophysical states. 
Table 6-3: Values of the similarity criteria for ducted flows with upstream-oriented gravitational acceleration. 154

Table 6-4: Values of the similarity criteria for ducted flows with downstream-oriented gravitational acceleration. 156

Table 6-5: Values of the similarity criteria for ducted flows with wall-normal-oriented gravitational acceleration. 157

\section{List of Figures}

Figure 1-1: (a) Plot of specific heat for water, $T_{c}=373.95 \mathrm{C}, p_{c}=22.064 \mathrm{MPa}$ (reproduced from Wagner \& Kruse (1997)); (b) Fluid properties of water at $24 \mathrm{MPa}$ in the vicinity of the pseudo-critical state (density - $\rho$, viscosity - $\mu$, thermal conductivity - $k$, and specific heat $-C_{p}$, of water. 3

Figure 1-2: Schematic of laminar-to turbulent transition from small disturbances over a flat surface. Adapted from Schlichting \& Gersten (2000).

Figure 1-3: Schematic of a hairpin vortex showing regeneration mechanisms (adapted from Brinkerhoff (2014)) 13

Figure 1-4: Visualization of a wavepacket of hairpin vortices adapted from the DNS study of Zhou et al. (1999); PHV - primary hairpin vortex; SHV - secondary hairpin vortex; THV - tertiary hairpin vortex; and DHV - downstream hairpin vortex, typically absent in fully-turbulent flows. 14

Figure 1-5: Schematic of nested hairpin vortex packets growing up from the wall (Adrian

et al., 2000). 17 
Figure 2-1: Convection heat transfer modes for ducted flows of single-phase subcritical fluid (Metaias \& Eckert, 1968); proposed SCWR operating conditions highlighted (Hofmeister et al., 2007).

Figure 2-2: Ratio of measured heat transfer coefficient to heat transfer coefficient calculated based the Dittus-Boelter correlation with water as the working fluid (Cheng \& Schulenberg, 2001). 28

Figure 2-3: Wall-normal distribution of streamwise velocity profile from experimental data of Kurganov \& Kaptilnyi’s (1993). 31

Figure 2-4: Comparison of LES results (Niceno \& Sharabi, 2013) with experimental data (Pis'menny et al. 2006). 35

Figure 4-1: Schematic of the computational domain for the turbulent flat-surface cases; $\mathrm{x}$-scale $/ \mathrm{y}$-scale $=2.5, \mathrm{x}$-scale $/ \mathrm{z}$-scale $=1$. 50

Figure 4-2: Node spacing in the wall-normal direction for the flat-surface cases. 54

Figure 4-3: (a) Mean velocity profiles; (b) Root-mean-square of the streamwise velocity fluctuation (c) Mean rate of turbulence kinetic energy production and dissipation. FP_QO $(\operatorname{Re} \delta 2=900)$, Spalart $(\operatorname{Re} \delta 2=1410), \mathrm{Wu} \& \operatorname{Moin}(\operatorname{Re} \delta 2=900)$ 58

Figure 4-4: Iso-surface of the second invariant of the velocity-gradient tensor, $q^{+}=0.01$, for $F P \_Q 0$, coloured by wall-normal height; $\operatorname{Re} \delta 2=930$. 59 Figure 4-5: Hairpin-like structures viewed via contours of instantaneous z-vorticity at plane $z / \delta=1.5 ; \operatorname{Re} \delta 2=930$. 60 Figure 4-6: Contours of z-vorticity for the heated turbulent flat-surface cases $\operatorname{Re}_{\tau}=250.63$ Figure 4-7: Streaks viewed through iso-contours of the instantaneous streamwise perturbation velocity component; turbulent flat-surface cases. $R e_{\tau}=250$. 63 
Figure 4-8: Iso-surfaces of the second invariant of the velocity-gradient tensor, $q^{+}=0.01$, coloured by wall-normal height; a) Case $F P \_Q 0.4$, b) Case $F P \_Q 0.6$. 64

Figure 5-1: Temperature dependence of density, $\rho$, dynamic viscosity, $\mu$, thermal conductivity, $k$, and isobaric specific heat, $C_{p}$, of water in the vicinity of the pseudocritical state. 66

Figure 5-2: Schematic of a hairpin vortex in wall-bounded shear layers and its induced motions (adapted from Brinkerhoff (2014)). 68

Figure 5-3: Computational domains: a) domain for generating initial conditions for heated-wall simulations; b) domain for channel flow with heated walls. 77

Figure 5-4: Two-point correlations of the streamwise component of fluctuation velocity for the non-buoyant variable-property case. 79

Figure 5-5: Mean flow properties evaluated at $x_{\delta}=9$ for the turbulent channel cases: a) streamwise velocity component; b) root-mean-square of perturbation velocity components; c) specific rates of turbulence kinetic energy production $\left(P^{+}\right)$and dissipation $\left(\varepsilon^{+}\right) ;$d) temperature. 87

Figure 5-6: Wall-normal profile of density and viscosity at $x_{\delta}=9$ : a) mean density; b) mean viscosity; c) rms perturbation density; d) rms perturbation viscosity.

Figure 5-7: Flood-plots of the thermophysical properties in the non-buoyant variableproperty case at $\mathrm{x}_{\delta}=9$. 91

Figure 5-8: Streaks in the heated-wall, fixed-property case: a) low-speed streaks visualized through iso-surfaces of the instantaneous streamwise component of perturbation velocity $\left(u^{\prime \prime}=-0.3\right)$ coloured by wall-normal height; b) low- and high-speed streaks visualized through iso-surfaces of $\left(v^{\prime \prime} T\right)^{+}=1 \times 10^{-4}$. 94 
Figure 5-9: Velocity streaks in the heated-wall, fixed-property case identified by: a) flood-plots of the instantaneous streamwise component of perturbation velocity, $u^{\prime{ }^{+}} ; \mathrm{b}-\mathrm{c}$ ) wall-normal distribution of $\bar{\lambda}_{P S D_{-} v^{\prime \prime}{ }_{-} 1}$, normalized by $\delta_{v}(\mathrm{~b})$ and $\delta(\mathrm{c})$. 94

Figure 5-10: Comparison of streamwise distribution of the viscous length scale for flows with fixed and variable thermophysical properties in the absence of buoyancy forces.... 97 Figure 5-11: Temperature dependence of density, dynamic viscosity, and kinematic viscosity in the vicinity of the pseudo-critical state. 97

Figure 5-12: Iso-contours of the second invariant of the velocity gradient tensor $\left(q^{+}=0.01\right.$ based on inflow wall variables) coloured by wall-normal height for: a) heated-wall, fixed property case; b) non-buoyant, variable-property case. 98 Figure 5-13: Flow development in the streamwise region where $\bar{T}_{w}$ is in the vicinity of the pseudo-critical temperature, observed through flood-plots of spanwise vorticity $\left(\omega_{z}\right)$ and velocity vectors in the: a) heated-wall, fixed-property case; b) non-buoyant, variableproperty case. 100

Figure 5-14: Flow development in the streamwise region where $\bar{T}_{w}$ is in the vicinity of the pseudo-critical temperature, observed through flood-plots of density and dynamic viscosity. 101

Figure 5-15: Ratio of the rms budgets of local rates of change of streamwise component of vorticity: $\left(P_{\omega, \text { baroclinic,rms }} / P_{\omega, \text { stretch,rms }}\right)_{x}$ (solid line); $\left(P_{\omega, \text { expand,rms }} / P_{\omega, \text { stretch,rms }}\right)_{x}$ (dashed line). 103

Figure 5-16. Flow development visualized through flood-plots of the streamwise component of perturbation velocity for: a) the heated-wall, fixed-property case; b) the non-buoyant, variable-property case. 105 
Figure 5-17: Streaky structures in the non-buoyant, variable-property case identified by: a) flood-plots of the instantaneous streamwise component of perturbation velocity, $u^{\prime \prime}$; bc) wall-normal distribution of $\bar{\lambda}_{P S D_{-} v_{-}{ }_{-} 1}$, normalized by $\delta_{v}(\mathrm{~b})$ and $\delta(\mathrm{c})$. 105

Figure 5-18. Illustration of the generation of high-speed fluid ejection motions along the wall of the non-buoyant, variable-property case: a-c) formation of a single hairpin vortex enveloping a high-speed streak; $\mathrm{d}-\mathrm{f}$ ) formation of a pair of hairpin vortices enveloping the downstream end of a high-speed streak. 107

Figure 5-19. Instantaneous velocity and vorticity flood-plots for the lower wall of the case with wall-normal gravitational acceleration: a) low- and high-speed streaks visualized through flood-plots of instantaneous streamwise perturbation velocity component; b) flood-plots of instantaneous wall-normal component of vorticity. 109 Figure 5-20. Wall-normal profile of the wall-normal temperature gradient for the nonbuoyant cases at $x_{\delta}=9$ plotted against: a) $y^{+}$; b) $y_{\delta}$.

Figure 5-21. Iso-surfaces of $\left(v^{\prime \prime} T\right)^{+}=1 \times 10^{-4}$ for: a) the heated-wall, fixed property case; b) non-buoyant, variable-property case.

Figure 5-22: Streamwise distribution of the normalized viscous length scale for the heated-wall, fixed-property case, non-buoyant, variable-property case, and the lower wall of the case with wall-normal gravitational acceleration. 113 Figure 5-23: Velocity streaks along the lower wall of the channel in the presence of wallnormal gravitational acceleration identified by: a) flood-plots of the instantaneous streamwise component of perturbation velocity, $\left.u^{\prime \prime} ; \mathrm{b}-\mathrm{c}\right)$ wall-normal distribution of $\bar{\lambda}_{P S D_{-} v_{-} 1}$, normalized by $\delta_{v}(\mathrm{~b})$ and $\delta(\mathrm{c})$. 114 
Figure 5-24: Illustration of the generation of vorticity through the baroclinic instability mechanism along the lower channel wall. 115

Figure 5-25: Flood-plots of density near the lower wall where the encircled regions are areas occupied by hairpin vortices. 115

Figure 5-26. Ratio of the rms budgets of local rates of change of streamwise component of vorticity at $x_{\delta}=9$ : a) Lower wall of the case with wall-normal gravitational acceleration; b) the non-buoyant, variable-property case.

Figure 5-27. Flood plots of the production of vorticity via the baroclinic mechanism at $x_{\delta}=9$ for the lower wall of the case with wall-normal gravitational acceleration and for the non-buoyant variable-property case.

Figure 5-28: Iso-surfaces of $\left(v^{\prime \prime} T\right)^{+}=1 \times 10^{-4}$ for: a) the non-buoyant, variable-property case; b) the lower wall of the case with wall-normal gravitational acceleration. 118 Figure 5-29: Wall-normal profile of the wall-normal temperature gradient for the nonbuoyant cases, and the lower wall of the case with wall-normal gravitational acceleration at $x_{\delta}=9$ plotted against: a) $y^{+}$; b) $y_{\delta}$ 118 Figure 5-30: Streamwise distribution of the normalized viscous length scale for the heated-wall, fixed-property case, the non-buoyant, variable-property case, and the upper wall of the case with wall-normal gravitational acceleration. 119 Figure 5-31: Velocity streaks on the upper wall of the channel in the presence of wallnormal gravitational acceleration identified by: a) flood-plots of the instantaneous streamwise component of perturbation velocity, $\left.u^{\prime \prime} ; \mathrm{b}-\mathrm{c}\right)$ wall-normal distribution of $\bar{\lambda}_{P S D_{-} v_{-} 1}$, normalized by $\delta_{v}(\mathrm{~b})$ and $\delta(\mathrm{c})$ 120 
Figure 5-32: Distribution of velocity streaks along the upper wall of the case with wallnormal gravitational acceleration visualized through flood-plots of the streamwise component of perturbation velocity.

Figure 5-33: Illustration of the generation of vorticity through the baroclinic instability mechanism along the upper wall of the case with wall-normal oriented gravitational acceleration. 122

Figure 5-34: Iso-surfaces of $\left(v^{\prime \prime} T\right)^{+}=1 \times 10^{-4}$ for: a) the non-buoyant, variable-property case; b) the upper wall of the case with wall-normal gravitational acceleration. 123 Figure 5-35: Wall-normal profile of the wall-normal temperature gradient for the nonbuoyant cases, and the upper wall of the case with wall-normal gravitational acceleration at $x_{\delta}=9$ plotted against: a) $y^{+}$; b) $y_{0}$ 123 Figure 5-36: Streamwise distribution of the normalized viscous length scale for the heated-wall, fixed-property case, the lower wall of the case with wall-normal gravitational acceleration, and the case with downstream-oriented gravitational acceleration. 125

Figure 5-37: Velocity streaks in the case with downstream-oriented gravitational acceleration identified by: a) flood-plots of the instantaneous streamwise perturbation velocity component, $u^{\prime \prime}$; b-c) wall-normal distribution of $\bar{\lambda}_{P S D_{-} v_{-}{ }_{1}}$, normalized by $\delta_{v}(\mathrm{~b})$ and $\delta(\mathrm{c})$. 125

Figure 5-38: Illustration of the generation of vorticity through the baroclinic mechanism in a channel with downstream-oriented gravitational acceleration 127

Figure 5-39: Iso-surfaces of $\left(v^{\prime \prime} T\right)^{+}=1 \times 10^{-4}$ for: a) the non-buoyant, variable-property case; b) the case with downstream-oriented gravitational acceleration. 127 
Figure 5-40: Wall-normal profile of the wall-normal temperature gradient for the nonbuoyant cases, and the case with downstream-oriented gravitational acceleration at $x_{\delta}=9$

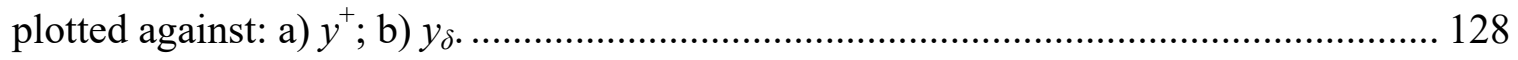

Figure 6-1: Variation of $\rho^{*}$ with $h^{*}$ for water, carbon dioxide, helium, and R134a in the vicinity of the pseudo-critical state. Data obtained from the NIST Standard Reference

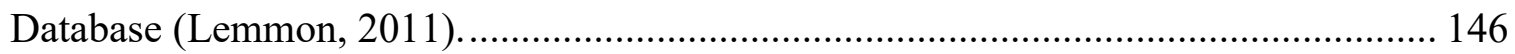
Figure 6-2: Variation of $\mu^{*}$ with $h^{*}$ for water, carbon dioxide, helium, and R134a in the vicinity of the pseudo-critical state. Data obtained from the NIST Standard Reference Database (Lemmon, 2011). 146

Figure 6-3: Variation of $k^{*}$ with $h^{*}$ for water, carbon dioxide, helium, and R134a in the vicinity of the pseudo-critical state. Data obtained from the NIST Standard Reference Database (Lemmon, 2011). 147

Figure 6-4: Variation of $C_{p}{ }^{*}$ with $h^{*}$ for water, carbon dioxide, helium, and R134a in the vicinity of the pseudo-critical state. Data obtained from the NIST Standard Reference Database (Lemmon, 2011). 147

Figure 6-5: State-space distribution of thermophysical properties based on three nondimensional independent state-space parameters: (a) $h^{*}=\left(\beta_{p c} / C_{p, p c}\right)\left(h-h_{p c}\right)$; (b) $T / T_{p c}$; (c) $\left(T-T_{p c}\right) /\left(T_{p c}-T_{c}\right)$. Data obtained from the NIST Standard Reference Database (Lemmon, 2011) 148

Figure 6-6: Variation of $\operatorname{Pr}$ with $h^{*}$ for water, carbon dioxide, helium, and R134a. Data obtained from the NIST Standard Reference Database (Lemmon, 2011). 149 
Figure 6-7: Values of similarity criteria for ducted flows with upstream-oriented gravitational acceleration, as per the studies listed in Table 6-3, showing ranges for which IHT and DHT are observed. 158

Figure 6-8: Published data assessed against pairs of similarity criteria for ducted flows with upstream-oriented gravitational acceleration. 160

Figure 6-9: Published data assessed against pairs of $Q^{*} \& p^{*}$ and $Q^{*} \& R i_{\text {in }}^{\prime}$ for ducted flows with downstream-oriented gravitational acceleration. 161

Figure 6-10: Published data assessed against pairs of $Q^{*} \& p^{*}$ and $Q^{*} \& R i_{\text {in }}$ for ducted flows with horizontal-oriented gravitational acceleration. 161

Figure 6-11: Streamwise variation of Nusselt number for pairs of working fluids at specific values of $Q^{*}$ and $p^{*}$ to yield: $(\mathrm{a}, \mathrm{b}) \mathrm{IHT} ;(\mathrm{c}, \mathrm{d})$ DHT. 163

Figure A-1: Schematic of the computational domain for the laminar cases. 193 Figure A-2: Computational domains for the turbulent channel cases: (a) computational domain for generating initial conditions for heated-wall channel flow simulations; (b) computational domain for channel flow with heated walls. 194

Figure A-3: Streamwise distribution on Nusselt number for fixed-fluid-property channel flows. 201

Figure A-4: Nusselt number distributions for a range of various heat-flux to mass-flux ratios in the laminar channel cases; Redh $=2000$. 202 Figure A-5: Effects of thermophysical property variations on the Nusselt number at two heat-flux to mass-flux ratios; $\operatorname{Redh}=2000$. 204 
Figure A-6: Wall-normal gradients of thermophysical properties for various cases at a

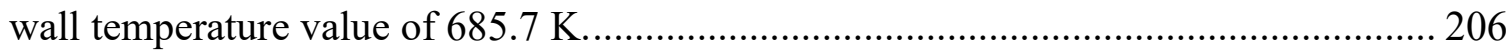

Figure B-1: Schematic of a two-dimensional mesh showing discretization nomenclature

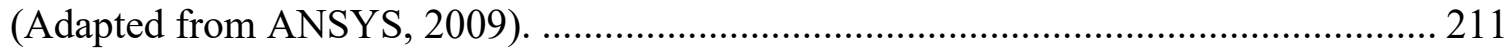




\section{Nomenclature}

\begin{tabular}{|c|c|}
\hline$A$ & Area $\left(\mathrm{m}^{2}\right)$ \\
\hline$A c$ & Acceleration parameter [Equation 5-1] \\
\hline$b$ & Coefficient vector [Equation B-14] \\
\hline Bo & Buoyancy parameter [Equation 5-2] \\
\hline$C_{p}$ & Isobaric specific heat $(\mathrm{J} / \mathrm{kg} \mathrm{K})$ \\
\hline $\mathrm{c}_{\#}$ & Empirical multiplier \\
\hline$C$ & Coefficient matrix [Equation B-14] \\
\hline$d_{h}$ & Hydraulic diameter (m) \\
\hline$G$ & Mass flux $\left(\mathrm{kg} / \mathrm{s} \mathrm{m}^{2}\right)$ \\
\hline$G r$ & Grashof number [Equation 6-10] \\
\hline$g$ & Gravitational acceleration $\left(\mathrm{m} / \mathrm{s}^{2}\right)$ \\
\hline$F$ & Empirically derived function \\
\hline$f$ & Force per unit volume $\left(\mathrm{N} / \mathrm{m}^{3}\right)$ \\
\hline$H$ & Convection heat transfer coefficient $\left(\mathrm{W} / \mathrm{m}^{2} \mathrm{~K}\right)$ \\
\hline$h$ & Static enthalpy $(\mathrm{J} / \mathrm{kg})$ \\
\hline$h_{0}$ & Stagnation enthalpy $(\mathrm{J} / \mathrm{kg})$ \\
\hline$K$ & Turbulence kinetic energy $\left(\mathrm{J} / \mathrm{m}^{3}\right)$ \\
\hline$k$ & Thermal conductivity (W/m K) \\
\hline$L$ & Length $(\mathrm{m})$ \\
\hline$M$ & Momentum source $\left(\mathrm{kg} / \mathrm{m}^{2} / \mathrm{s}^{2}\right)$ [Equation B-2] \\
\hline$m$ & Mass (kg) \\
\hline$N$ & Shape function [Equation A-1] \\
\hline
\end{tabular}




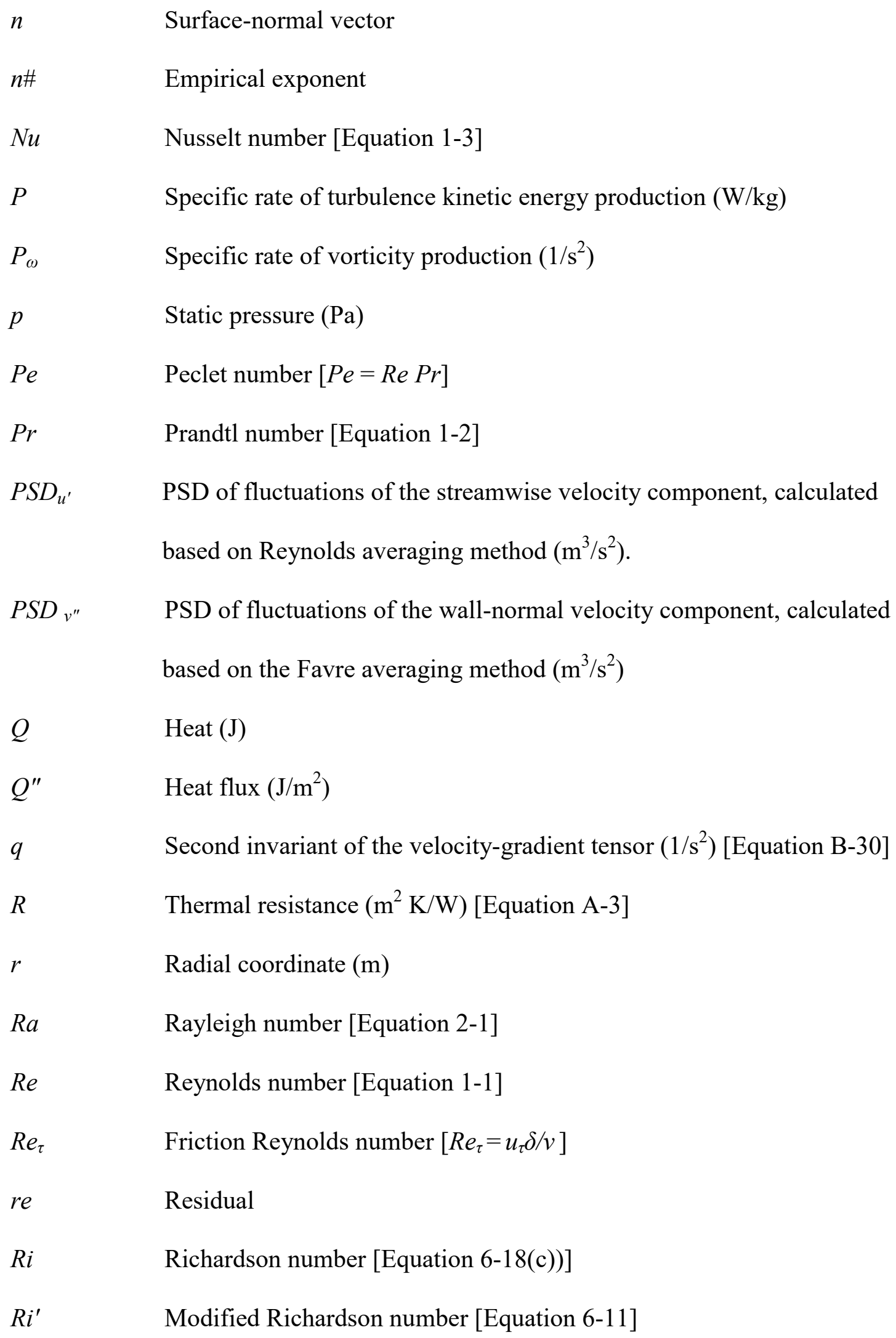




\begin{tabular}{|c|c|}
\hline$T$ & Temperature $(\mathrm{K})$ \\
\hline$T_{\tau}$ & Friction temperature $(\mathrm{K})\left[T_{\tau}=\dot{Q}_{w}^{\prime \prime} /\left(\rho_{w} C_{p, w} u_{\tau}\right)\right]$ \\
\hline$t$ & Time (s) \\
\hline$t_{0}$ & Reference time (s) \\
\hline$t_{\eta}$ & Kolmogorov time scale (s) [Equation 4-3] \\
\hline$u$ & Streamwise velocity $(\mathrm{m} / \mathrm{s})$ \\
\hline$u_{i}$ & $i$ Cartesian component of velocity $(\mathrm{m} / \mathrm{s})$ \\
\hline$u_{\tau}$ & Friction velocity $(\mathrm{m} / \mathrm{s})\left[u_{\tau}=\left(\tau_{w} / \rho_{w}\right)^{0.5}\right]$ \\
\hline$u_{\eta}$ & Kolmogorov velocity scale $(\mathrm{m} / \mathrm{s})$ [Equation 1-8] \\
\hline$u^{0}$ & Velocity at time $t_{0}(\mathrm{~m} / \mathrm{s})$ \\
\hline$V$ & Volume $\left(\mathrm{m}^{3}\right)$ \\
\hline$v$ & Wall-normal velocity $(\mathrm{m} / \mathrm{s})$ \\
\hline$w$ & Spanwise velocity $(\mathrm{m} / \mathrm{s})$ \\
\hline$x$ & Streamwise coordinate $(\mathrm{m})$ \\
\hline$x_{i}$ & $i$ Cartesian spatial coordinate $(\mathrm{m})$ \\
\hline$y$ & Wall-normal coordinate $(\mathrm{m})$ \\
\hline$z$ & Spanwise/transverse coordinate $(\mathrm{m})$ \\
\hline
\end{tabular}

\section{Greek Symbols}

$\alpha \quad$ Thermal diffusivity $\left(\mathrm{m}^{2} / \mathrm{s}\right)$

$\beta \quad$ Volumetric expansion coefficient (1/K)

$\delta \quad$ Boundary layer thickness (m)

$\delta_{2} \quad$ Momentum thickness (m) 


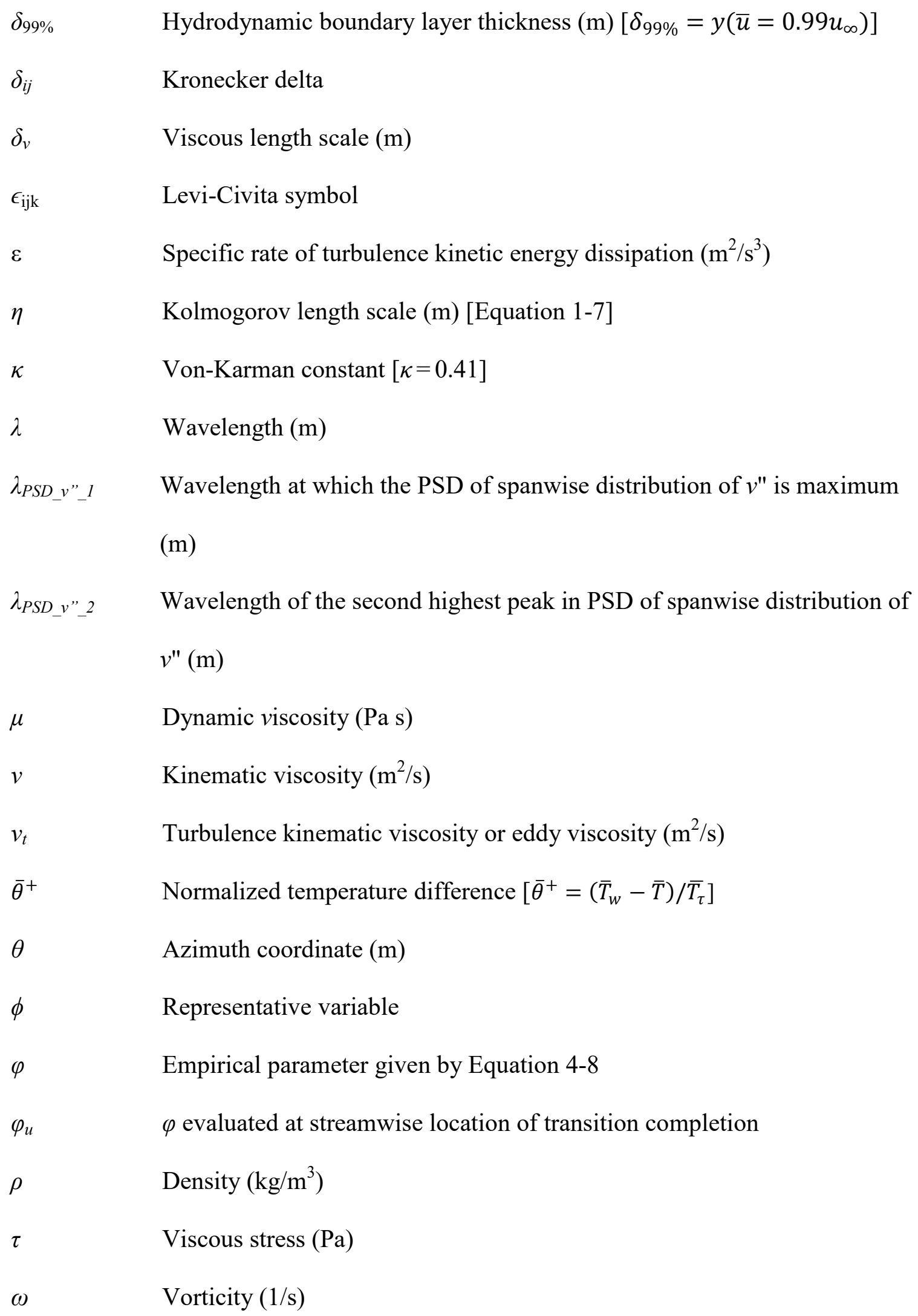




\section{Subscripts}

\begin{tabular}{|c|c|}
\hline$\infty$ & Free-stream conditions \\
\hline 0 & Stagnation value \\
\hline$B$ & Related to buoyancy forces \\
\hline$b$ & Bulk fluid \\
\hline baroclinic & Related to the baroclinic force \\
\hline$c$ & Thermodynamic critical state \\
\hline $\operatorname{conv}$ & Related to convection heat transfer \\
\hline crit & Critical value for stability \\
\hline$c v$ & Control volume \\
\hline$d_{h}$ & Based on the hydraulic diameter \\
\hline expand & Related to fluid expansion/compression \\
\hline$f$ & At film conditions \\
\hline flow & Normal to the flow direction \\
\hline$H T$ & Heat transfer \\
\hline$i$ & Tensor index \\
\hline$I$ & Related to inertia force \\
\hline ip & Integration point \\
\hline in & Inflow value \\
\hline$j$ & Tensor index \\
\hline$k$ & Tensor index \\
\hline$k$ & Related to the thermal conductivity \\
\hline
\end{tabular}




\begin{tabular}{|c|c|}
\hline$M$ & Modelling fluid \\
\hline out & Outflow value \\
\hline$P$ & Prototypical fluid \\
\hline$p c$ & Thermodynamic pseudo-critical state \\
\hline ref & Reference or characteristic value \\
\hline$r m s$ & Root mean square \\
\hline$s$ & Surfaces of the control volume \\
\hline stretch & Related to stretching/tilting of vorticity \\
\hline$t$ & Turbulence \\
\hline thermal & Related to the thermal boundary layer \\
\hline up & Upstream integration point \\
\hline velocity & Related to the hydrodynamic boundary layer \\
\hline viscous_ & Related to viscous diffusion \\
\hline diffusion & \\
\hline$w$ & At the surface/wall \\
\hline$x$ & Streamwise direction \\
\hline$y$ & wall-normal direction \\
\hline$z$ & Spanwise direction \\
\hline$\delta$ & Normalization by $\delta$ \\
\hline$\delta_{2}$ & Normalization by momentum thickness \\
\hline$\eta$ & Kolmogorov scale \\
\hline$\mu$ & Related to viscous force \\
\hline$\omega$ & Related to the vorticity field \\
\hline
\end{tabular}




\section{Superscript}

$*$

"l

$+$

$n$

$m$

$t$

\section{Accents and other symbols}

index

index

$\wedge$

\langle\rangle

\section{Acronyms}

2D

$3 \mathrm{D}$

AIP

CFD

DHV

DHT

Non-dimensional form of a parameter

Reynolds perturbation/fluctuation component

Favre perturbation/fluctuation component (Area flux when used with $Q$ )

Normalization with $u_{\tau}$ and $\delta_{v}$

Timestep index

Mean (values averaged over coordinates with statistically homogeneous

turbulence)

Density-weighted mean value

Time rate of change

Maximum value

Value that is time- and area-averaged over the spanwise/wall-normal plane

Two-dimensional

Three-dimensional

American Institute of Physics

Computational Fluid Dynamics

Downstream Hairpin Vortex

Deteriorated Heat Transfer 


\begin{tabular}{ll} 
DNS & Direct Numerical Simulations \\
FANS & Favre Averaged Navier-Stokes \\
GIF & Generation IV International Forum \\
IAPWS & International Association for the Properties of Water and Steam \\
IHT & Improved Heat Transfer \\
ILU & Incomplete Upper Lower \\
LES & Large Eddy Simulation \\
NHT & Normal Heat Transfer \\
NIST & National Institute of Standards and Technology \\
PHV & Primary Hairpin Vortex \\
PIV & Particle Image Velocimetry \\
PSD & Power Spectral Density \\
RANS & Reynolds Averaged Navier-Stokes \\
RSM & Reynolds Stress Model \\
SCWR & SuperCritical Water-cooled Reactor \\
SGS & Sub-Grid Scale \\
SHV & Secondary Hairpin Vortex \\
SST & Shear Stress Transport \\
THV & Tertiary Hairpin Vortex \\
T-S & Tollimen-Schlichting \\
WALE & Wall-Adapting Local Eddy-viscosity \\
ZPGB & Zero-streamwise-Pressure-Gradient turbulent Boundary \\
\hline
\end{tabular}




\section{Chapter: Outline and Background}

It has long been established that the heat transfer characteristics for flows of fluids at the supercritical thermodynamic state may significantly deviate from established patterns for flows of single-phase subcritical fluids. The present work strives to reconcile the fluid dynamics that yields these heat transfer characteristics. Readers who are subject-matter experts on the fluid mechanics and forced-convection heat transfer characteristics of turbulent flows may proceed to the general introduction presented in Chapter 2, as the literature reviews presented in Chapters 4, 5, and 6 would provide sufficient background. The remainder of the present chapter provides introductory information on the supercritical thermodynamic state, reviews the present understanding of fluid flow and convection heat transfer, and provides a framework for the rest of the thesis manuscript in the context of these topics. Chapter 2 provides a general introduction to the research topic of the present study supported by a brief review of the relevant literature. The objective of the thesis is outlined in Chapter 3. Chapters 4, 5, and 6 detail the approaches, analyses, and results that address the objectives of the thesis. In keeping with the integrated thesis format, these chapters are respectively based on the following articles:

1. Azih, C. Brinkerhoff, J.R. \& Yaras, M.I., 2012, 'Direct numerical simulation of convective heat transfer in a zero-pressure-gradient boundary-layer with supercritical water', Journal of Thermal Science, 21, pp.49-59.

This article received a best paper designation at the $10^{\text {th }}$ International Symposium on Experimental Computational Aerothermodynamics of Internal Flows held in Brussels, Belgium. 
2. Azih, C. \& Yaras, M.I., 2018, 'Effects of spatial gradients in thermophysical properties on the topology of turbulence in heated channel flow of supercritical fluids', Physics of Fluids, 30(1), p.015108, 23 pages.

This article was declared by the journal editors to be one of the top articles in Physics of Fluids, and was accordingly designated as a featured article.

3. Azih, C. \& Yaras, M.I., 2017, 'Similarity criteria for modelling mixed-convection heat transfer in ducted flows of supercritical fluids', Journal of Heat Transfer, 139(12), p.122501, 13 pages.

Chapter 7 provides a summary of the key conclusions drawn from the present work and how the objectives set out in Chapter 3 have been addressed. Recommendations of potential areas for further research effort are also presented in this chapter.

\subsection{The Supercritical Thermodynamic State}

Conventionally, the supercritical thermodynamic state is constrained by temperatures and pressures of $T>T_{c}$ and $p>p_{c}$, respectively. Here, the subscript $c$ indicates that properties are evaluated at the thermodynamic critical state. However, the temperature criterion that defines the supercritical state is often dismissed for heat transfer studies (Pioro \& Duffy, 2005); this convention is adopted in the present study. A key feature of the supercritical state is the presence of a pseudo-critical state, defined by the temperature at which the specific heat, $C_{p}$, is at its maximum value for a given pressure; this state is hereafter denoted by the subscript $p c$. Figure 1-1(a) illustrates the distribution of $C_{p}$ in state space for water. In this figure, the global maximum in $C_{p}$ represents the critical state. For a 
given isobar, the local maxima in $C_{p}$ observed for supercritical $\left(p>p_{c}\right)$ and subcritical $\left(p<p_{c}\right)$ states represent the pseudo-critical and two-phase states of water, respectively. Two notable characteristics particular to supercritical fluids are that: there is no equilibrium condition where liquid and gaseous fluid phases simultaneously exist; and, in the vicinity of the pseudo-critical state, the thermophysical properties of the fluid vary significantly with small variations in temperature, as illustrated in Figure 1-1(b). It is in the vicinity of the pseudo-critical state that unique flow and heat transfer characteristics are observed.

(a)

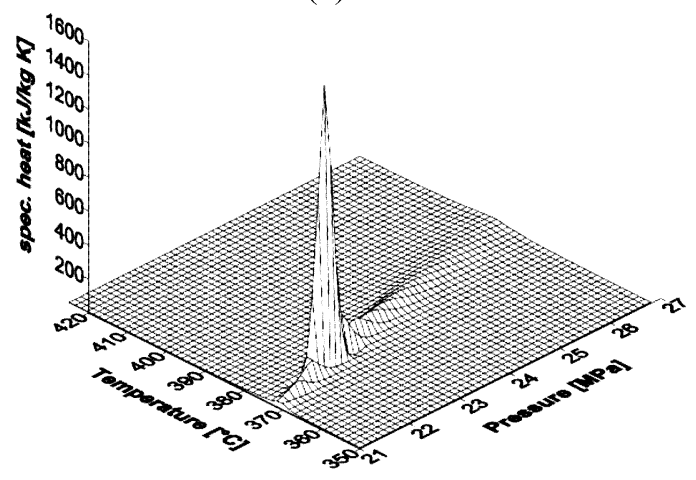

(b)

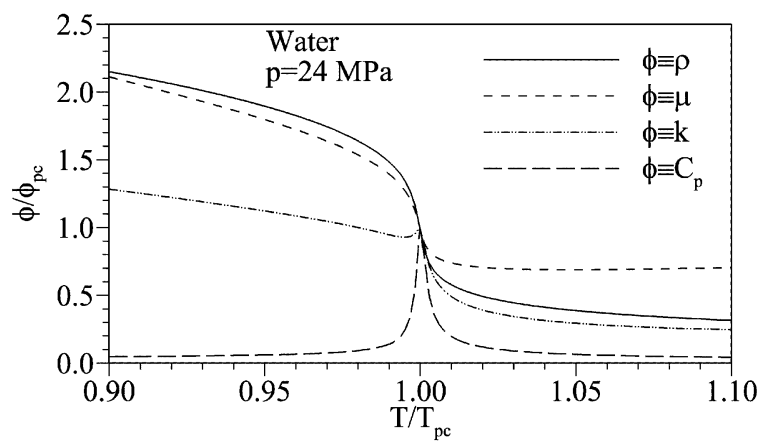

Figure 1-1: (a) Plot of specific heat for water, $T_{c}=373.95 \mathrm{C}, p_{c}=22.064 \mathrm{MPa}$ (reproduced from Wagner \& Kruse (1997)); (b) Fluid properties of water at $24 \mathrm{MPa}$ in the vicinity of the pseudo-critical state (density $-\rho$, viscosity - $\mu$, thermal conductivity - $k$, and specific heat $-C_{p}$, of water.

\subsection{Background on Fluid Mechanics and Heat Transfer}

The fluid mechanics and heat transfer of incompressible unheated wall-bounded flows of Newtonian working fluids through simple geometries is well established, and is covered with sufficient detail in many texts (e.g. Schlichting \& Gersten (2000) and White (1991)). In the interest of preserving its focus, this document provides only a brief background on 
fundamental fluid mechanics and heat transfer topics that are of high relevance to the present study. Readers are encouraged to consult one of the referenced texts should they require a more thorough treatment of these topics.

\subsubsection{Fluid Flows within Wall-Bounded Geometries}

Generally, flow systems feature transport mechanisms that temporally drive them towards a state of spatial uniformity. For example, a system exhibiting spatial gradients in density, temperature, and velocity, may respectively feature transports of mass, thermal energy, and momentum, such that the system tends towards a more spatiallyuniform distribution of the relevant parameters. In flows that have velocity gradients, momentum transport, via diffusion, occurs as a result of shear forces; thus, such flows are denoted as shear flows. Wall-bounded shear flows, which have one or more solid surfaces that define the flow geometry, can be categorized into internal and external flows. External flows are semi-bounded by walls; for example, flow over a flat surface or an airfoil. In contrast, internal flows are fully bounded by walls, such as flows within ducts. At the wall, a no-slip condition exists such that the fluid is stationary relative to the wall. With increasing distance from the wall, the local fluid velocity is less affected by the presence of the wall and may be more strongly influenced by other forces acting within the flow. This may create flow topology where appreciable velocity gradients, and hence shear forces, exist in a near-wall region of the flow. This region is ascribed as a wall-bounded shear layer or hydrodynamic boundary layer, which is characterized by length-scales representing distances from the wall. These length scales are denoted as boundary layer thicknesses, $\delta$. A shear flow may become unstable and permit spatial and temporal growth of prevailing disturbances, causing the flow to transition from a laminar 
state with orderly and well organized streamlines to a turbulent state featuring more complex and transient flow patterns. The stability of a shear flow is often primarily characterized by the ratio of inertial to shear (or viscous) forces, which is expressed through the Reynolds number, $R e$ :

$$
R e=\frac{\rho u_{r e f} L_{r e f}}{\mu}
$$

where $\rho$ is the fluid density, $u$ is the fluid velocity, $L$ is a relevant length scale, $\mu$ is the fluid dynamic viscosity, and the subscript ref indicates a characteristic/reference value for the flow. Linear stability analyses of wall-bounded shear flows reveal a critical Reynolds

number above which disturbances in the shear flow are amplified, yielding transition of the flow from a laminar state to a turbulent state (Kachanov, 1994). As opposed to laminar flows, where wall-normal transport of mass, momentum, and energy are realized primarily through molecular diffusion, transport of these parameters in turbulent flows occurs primarily through advection mechanisms. As a consequence, heat transfer rates are greatly affected by whether the flow is laminar or turbulent.

\subsubsection{Forced Convection Heat Transfer in Wall-Bounded Flows}

In this subsection the forced convection heat transfer characteristics for flows of singlephase subcritical fluids is reviewed to establish a baseline for the discussions presented in subsequent chapters.

At the wall, the fluid temperature matches that of the wall. As wall proximity decreases temperature approaches the bulk-fluid/free-stream temperature. In heated flows, this topology imposes a thermal boundary layer region in like manner to the hydrodynamic 
boundary layer. The ratio of the scales of these boundary layers may be characterized by the Prandtl number, $P r$, which represents a ratio of momentum- and thermal-diffusion rates, and is expressed as:

$$
\operatorname{Pr}=\frac{v}{\alpha}=\frac{\mu / \rho}{k /\left(\rho C_{p}\right)}=\frac{\mu C_{p}}{k}
$$

Here, $v$ is the kinematic viscosity (momentum diffusivity), $\alpha$ is the thermal diffusivity, and $k$ is the thermal conductivity. A convection heat transfer coefficient $(H)$, defined as the ratio of the surface heat flux to the temperature difference between the wall and the bulk fluid, may be used to characterize the rate of heat transfer. The $H$ value may be nondimensionalized in the form of the Nusselt number, $N u$, expressed as:

$$
N u=\frac{H L_{r e f}}{k}
$$

Turbulent wall-bounded flows typically have $N u$ values that are several orders of magnitude higher than those of equivalent laminar flows (Rohsenow et al., 1998), which reflects the dominant contribution of advection mechanisms to wall-normal heat transfer. The $N u$ value for single-phase turbulent ducted flows of subcritical fluids may be computed using the Dittus-Boelter correlation, expressed by Equation 1-4, with $c_{0}=0.023, n 1=0.8, n 2=0.4$ and $F=1$ (Winterton, 1998).

$$
N u=c_{0} \operatorname{Re}^{n 1} \operatorname{Pr}^{n 2} F
$$

Here, $c_{0}, n 1$, and $n 2$ are empirically derived constants, and $F$ is an empirically-derived function. In Equation 1-4, $\operatorname{Re}$ and $\operatorname{Pr}$ are evaluated at bulk-fluid conditions, and the length scale used to evaluate $R e$ is the hydraulic diameter, $d_{h}$. 
If the heat transfer rate is sufficiently high to impose significant spatial gradients of temperature, variations in the thermophysical properties may affect the fluid dynamics such that the Dittus-Boelter equation no longer adequately captures the heat transfer rate.

For example, Sieder $\&$ Tate propose a correction function, $F=\left(\mu_{b} / \mu_{w}\right)^{0.14}$, to account for significant changes in dynamic viscosity with temperature, where subscripts $w$ and $b$ indicate wall and bulk-fluid conditions, respectively (Sleicher \& Rouse, 1975). Flows of supercritical fluids may feature significant changes in thermophysical properties with relatively small changes in temperature. This could lead to spatial gradients of the thermophysical properties that are orders of magnitude higher than those observed in subcritical fluid flows such that correlations in the form of Equation 1-4 fail to adequately capture the heat transfer characteristics. This inadequacy is observed to be due to changes in the physical mechanisms that drive the local turbulence (Bae et al., 2005).

\subsection{Physical Mechanisms that Drive Turbulent Flows and Heat Transfer}

It was mentioned that advection due to turbulence motions causes increased rates of heat transfer in turbulent flows when compared to a laminar scenario. In this section, the topology of turbulence is discussed from the perspective of transition from laminar to turbulent flow regimes. This will provide a platform for a discussion of the present understanding of physical mechanisms that drive fully-turbulent flows through wallbounded geometries as per the introductory subsections in Chapters 4 and 5 . 
Transition to turbulence is a complex process that continues to be actively investigated as it has an impact on numerous engineering processes. For the purposes of this review, investigations for flows over zero-pressure-gradient boundary layers (ZPGBL) and ducted flows of uniform cross-section are considered. Also, only aspects of this subject that are deemed to be of direct significance to the statistically-steady fully-turbulent wallbounded shear layer are focused on in this review. Under these conditions, the flow developments observed in these two geometries are sufficiently similar that they can be addressed simultaneously (Schlichting \& Gersten, 2000). Any notable differences due to geometrical effects will be highlighted where necessary.

\subsubsection{Laminar-to-Turbulent Flow Transition in Unheated Flows}

Flow at a laminar state may permit spatial and temporal growth of disturbances if the local Reynolds number exceeds the critical Reynolds number $\left(R e_{c r i t}\right)$ of the flow. The growth and development of these disturbances provide a framework for transition to turbulence. In flow environments with low disturbance levels, transition occurs through linear amplification of two-dimensional (2D) Tollmien-Schlichting waves (T-S waves) that develop via a viscous-instability mode as illustrated in Figure 1-2. As the T-S waves amplify and convect downstream, a secondary instability yields a non-linear growth phase which manifests in the form of spanwise-waviness of the streamwise-grouped spanwise vorticity (Kachanov, 1994). The self-induced effects of this vorticity topology result in lift-up away from the wall to form three-dimensional (3D) $\Lambda$-shaped vortices as schematically depicted in Figure 1-2 (Klebanoff \& Tidstrom, 1959). These vortices eventually develop into turbulent spots through self and mutual induction, replication, and 3D non-linear interactions with the base flow. Amalgamation of several instances of 
the turbulent spots results in a fully-turbulent flow containing self-sustaining vortical flow structures.

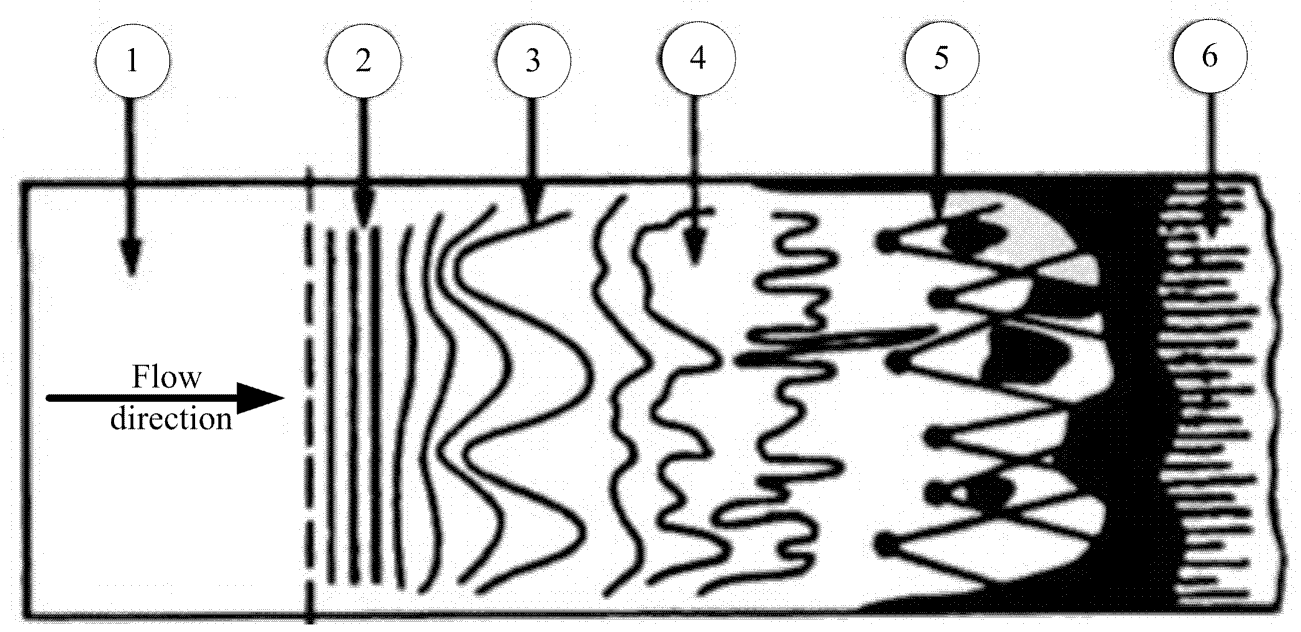

(1) Stable laminar flow; (2) Unstable T-S waves; (3) $\Lambda$-shaped vortices

(4) Breakdown of $\Lambda$-shaped vortices (5) Formation of turbulent spot (6) Fully turbulent flow

Figure 1-2: Schematic of laminar-to turbulent transition from small disturbances over a flat surface. Adapted from Schlichting \& Gersten (2000).

Besides the sequences of event described in the foregoing, alternative mechanisms for transition to turbulence may occur. For example, Rayleigh (1879) showed the presence of an inflectional velocity profile in a boundary layer to be a sufficient condition for flow instability in a 2D inviscid mode. The 2D inviscid instability causes the shear layer to roll up into discrete vortices oriented normal to the 2D plane (Drazin, 2002). Accordingly, should an inflectional profile spawn in the streamwise/wall-normal plane of a wallbounded flow, streamwise grouping of spanwise vorticity may occur due to this inviscidinstability mode, which then roll up into $\Lambda$-shaped vortices. A different route to transition may also occur in flows with high disturbance levels, for example, flows with elevated 
free-stream turbulence. In such flows, 3D instability modes develop nearly instantaneously, bypassing the 2D phases of transition; thus, this mode is denoted bypass transition (Wu \& Moin, 2009, Brinkerhoff \& Yaras, 2015).

The disturbances that initiate the laminar-to-turbulent transition process may originate from several sources, e.g. free-stream turbulence, surface roughness, surface vibration, or acoustic sources. Regardless of the nature and strength of the initial disturbance that triggers transition or the route that the flow takes to transition, given sufficient development, the flow reaches a fully-turbulent state that is independent of the triggering disturbance(s) (Fathali et al., 2007). In the ZPGBL, the resulting fully-turbulent flow is statistically homogeneous in the temporal and spanwise coordinates, whereas, for ducted flows statistical homogeneity occurs in the temporal, streamwise, and spanwise coordinates. Notwithstanding these differences, the flow structures of turbulence are observed to develop in a similar fashion in ZPGBL and ducted flows (Robinson, 1991).

\subsubsection{Effect of Heat Transfer on Laminar-to-Turbulent Flow Transition}

Spatial gradients of temperature may affect the process of transition to turbulence through associated spatial gradients of the fluid properties. Particular focus has been directed towards studying the effects of such gradients on the linear-growth phase of transition, which is governed by the fourth-order differential Orr-Sommerfeld equation for flows without such spatial gradients (Schlichting \& Gersten, 2000). Wazzan et al. (1972) numerically investigated the effect of wall-normal gradients of dynamic viscosity, $\mu$, on the stability of a ZPGBL, using water as the working fluid and in the absence of buoyancy forces. Their study was conducted at atmospheric pressure over a temperature 
range of $1^{\circ} \mathrm{C}$ to $90^{\circ} \mathrm{C}$. They modified the Orr-Sommerfeld equation to account for spatial gradients in $\mu$. Wazzan et al.'s (1972) analysis imposed two-dimensional perturbations on the base flow, which can be more effective in initiating transition than three-dimensional disturbances (Schafer et al., 1995). They observed that when $\mu$ decreases with wall proximity, the net effect was to reduce $R e_{c r i t}$. This wall-normal viscosity profile has been demonstrated analytically to enforce an inflection point in the wall-normal profile of the streamwise velocity component (Schlichting \& Gersten (2000)), which is inviscidunstable as previously discussed. Schafer et al. (1995) studied the effects of small temperature-induced variations in $\rho, \mu, k$, and $C_{p}$ on the boundary layer stability in the absence of buoyancy forces. The methodology involved supplementing modified OrrSommerfeld equations with a linearized thermal energy equation to capture the development of the disturbance energy. These authors observed that the contributions of variations of the hydrodynamic parameters $(\rho$ and $\mu$ ) on the stability of the hydrodynamic boundary layer significantly outweigh those of the thermal parameters $\left(k\right.$ and $\left.C_{p}\right)$ by several orders of magnitude. Their observation on the effect of dynamic viscosity compared favourably with those of Wazzan et al. (1972). Additionally, Schafer et al. (1995) observed that when $\rho$ decreased as the wall was approached the flow was stabilized. Their study was extended by Ozgen (2004) to include flows with large gradients of the thermophysical properties. His computations involved solving the OrrSommerfeld equations modified to allow for large gradients in $\mu$ and $\rho$. Based on his results, Ozgen (2004) argued that in the absence of gravitational acceleration, the kinematic viscosity, $v=\mu / \rho$, would have a dominant influence on the stability of the flow. The wall-normal distribution of $v$ was observed to have a three-fold effect on the stability 
of the boundary layer. First, it may impose an inflection point in the velocity profile of the boundary layer, as per the observations of Wazzan et al. (1972) and Schafer et al. (1995), which is inviscid-unstable. Also, it acts to diffuse vorticity created in high-shear regions near the wall, and thus contributes to destabilization of the flow. Finally, the kinematic viscosity also promotes the dissipation of kinetic energy of disturbances, which has a stabilizing influence on the flow. The net stabilizing/destabilizing effect is determined by the dominant of these three modes which may be unique to each profile of velocity and wall-normal kinematic viscosity gradient. When the spatial gradients in the thermophysical properties are small, their effect on the topology of the fully-turbulent state is observed to also be small. On the other hand, larger spatial gradients of the thermophysical properties have been observed to significantly influence the topology of turbulence (Bae et al., 2005).

\subsubsection{Coherent Structures of Turbulence}

A primary hairpin vortex may form in close proximity to the wall through streamwise grouping and subsequent roll-up of spanwise vorticity driven by inviscid and/or viscous flow instabilities (Kachanov, 1994). From experiments and simulations of turbulent spots, multiple mechanisms have been observed by which a mature hairpin vortex may create secondary hairpin vortices. Zhou et al. (1999) and Singer \& Joslin (1994) noted that mutual induction by the streamwise legs of the hairpin vortex causes motion away from the wall, and yields stretching of the vortex in the streamwise direction under action of the background shear. These authors proposed that a secondary vortex is formed between the legs of a parent vortex through a combination of self-induced motions due to the presence of the wall, the local curvature of the vortex-core centreline, and the inviscid 
instability of the separated shear layer that forms behind the primary/parent vortex as indicated in Figure 1-3. Likewise, the secondary hairpin vortex may create a tertiary hairpin vortex through the same mechanism, and so on. This sequence of hairpin-vortex generation produces a group of streamwise-aligned vortices denoted as a wavepacket (Adrian et al. 2000). A sample wavepacket observed in the DNS results of Zhou et al. (1999) is illustrated in Figure 1-4, where a disturbance is allowed to develop in a background flow representative of the mean streamwise velocity of a fully-turbulent channel flow. Schröder et al. $(2008,2004)$ and Bernard et al. (1993) suggest an alternative mechanism for the formation of secondary hairpin vortices which results in them straddling the legs of the parent hairpin vortex. As indicated in Figure 1-3, the topology and orientation of vorticity of the hairpin vortex is such that fluid is transported

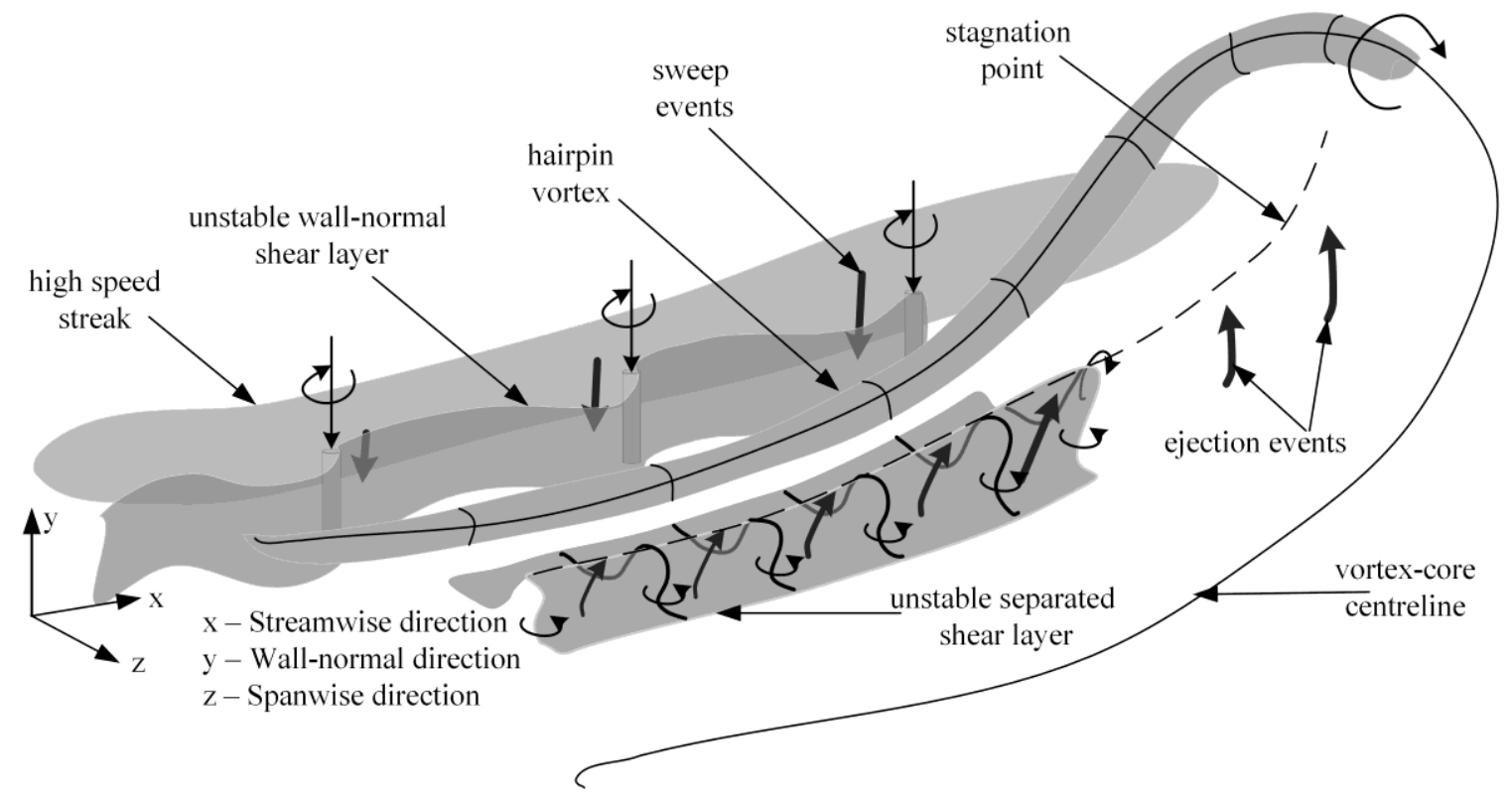

Figure 1-3: Schematic of a hairpin vortex showing regeneration mechanisms (adapted from Brinkerhoff (2014)) 


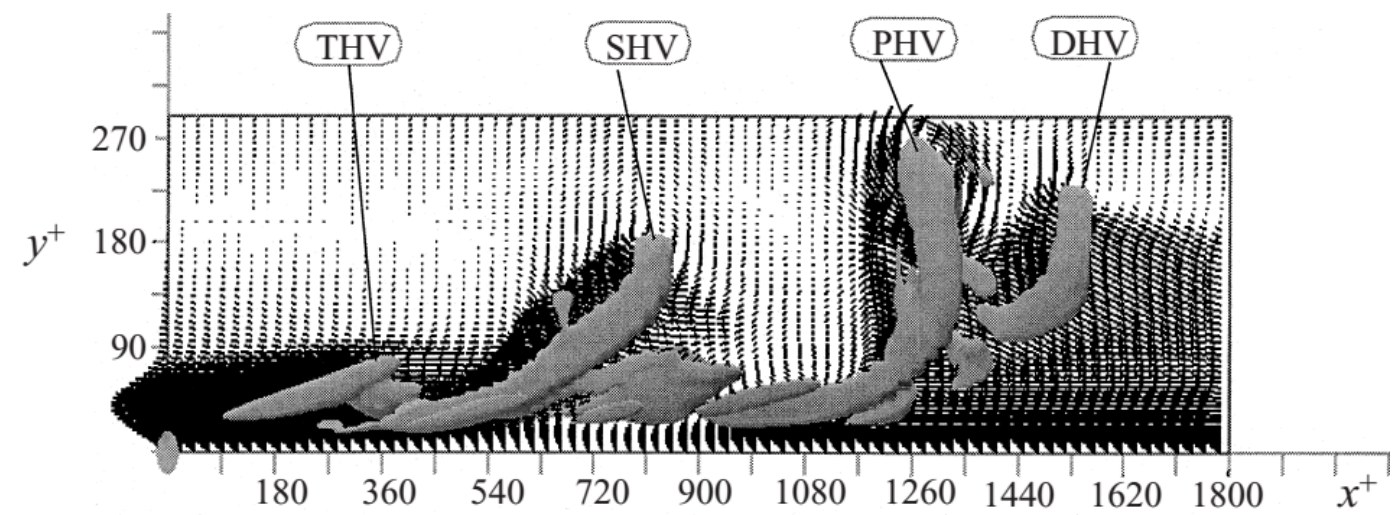

Figure 1-4: Visualization of a wavepacket of hairpin vortices adapted from the DNS study of Zhou et al. (1999); PHV - primary hairpin vortex; SHV - secondary hairpin vortex; THV - tertiary hairpin vortex; and DHV - downstream hairpin vortex, typically absent in fully-turbulent flows.

away from the wall (ejection) between the legs of the vortex, and transferred towards the wall (sweep) on the outboard sides of the legs. Due to the mean shear imposed on the flow by the presence of the wall, these ejection and sweep motions give rise to localized streamwise-aligned regions of streamwise momentum excess and deficit that are termed high- and low-speed streaks, respectively. Secondary instability of these streaks provides a route for generation of secondary hairpin vortices. The streamwise velocity profile in close vicinity of the high- and low-speed streaks is inflectional in the spanwise and wallnormal directions, and may result in localized inviscid instabilities of the streak, which respectively manifest in two modes: an asymmetric sinuous mode occurs in the form of streamwise-periodic oscillations of the streaks in the streamwise/spanwise plane, while a symmetric varicose instability occurs in the form of streamwise-periodic widening and narrowing of the streak (Andersson et al., 2001; Skote et al., 2002). In particular, the varicose instability mode is noted to yield streamwise-periodic accumulation of wall 
normal vorticity, as per Figure 1-3, which roll up into discrete wall-normal vortices. (Skote et al. 2002, Brinkerhoff \& Yaras, 2015).

The rolled-up vortices deform around the neighbouring leg of the parent vortex and form a wavepacket of streamwise-aligned vortices that straddle the leg of the parent vortex. (Schröder et al., 2008; Bernard et al., 1993). The hairpin vortices in such wavepackets are created almost simultaneously, unlike those in the alternate mechanism where new hairpin vortices appear to be created sequentially. Notably, the foregoing studies on the regeneration mechanisms of hairpin vortices have been mostly limited to young turbulent spots with only a few generations of hairpin vortices. In fully-turbulent flows, secondary hairpin vortices have to compete with existing vortices, thus one regeneration mechanism may be favoured over the other. Brinkerhoff \& Yaras (2014) studied the regeneration mechanisms of hairpin vortices in a mature turbulent spot that contained more than four generations of hairpin vortices. The authors observed that when the legs of the parent hairpin vortices are more closely spaced in the spanwise direction, the secondary hairpin vortices form between the legs of the primary hairpin vortices, whereas parent hairpin vortices with larger spanwise spacing between their legs spawn secondary vortices straddling the legs of the parent vortices. The dense population of vortices in fullyturbulent flows may preferentially promote the development of hairpin vortices having narrower spacing between their respective legs and thus preferentially promote the creation of secondary hairpin vortices in sequence behind the primary hairpin vortex (Adrian et al., 2000; Tomkins \& Adrian, 2003). 
Within a wavepacket, the older, and hence taller, vortices extend to regions of higher velocity in the boundary layer, and therefore tend to convect downstream at higher speeds than the younger vortices. As such, streamwise interaction of neighbouring hairpin vortices within a wavepacket is rare (Adrian et al., 2000; Tomkins \& Adrian, 2003). On the other hand, complex interactions are observed between wavepackets and their hairpin vortices, which are primarily promoted by the spanwise growth and spanwise meandering of the wavepackets, and by the streamwise interaction of wavepackets due to their varying convective speeds. For example, the adjacent legs of spanwise-interacting hairpin vortices may break down into smaller vortical structures owing to their opposite directions of rotation while the heads may merge to form a hairpin vortex of greater spanwise extent (Adrian et al., 2000; Tomkins \& Adrian, 2003). Tomkins \& Adrian (2003) propose that as the heads of these vortices merge, the low speed streaks associated with the wavepackets of the merging vortices also merge and form a single low-speed streak with a larger spanwise width. These authors noted that most of the near-wall lowspeed streaks, observed through their Particle Image Velocimetry (PIV) measurements in a fully-turbulent boundary layer, had begun to undergo this merging process by a wallnormal height of $y^{+}=100$. Here, the "+" superscript denotes wall units, i.e. normalization by wall variables, $v_{w}$ and $u_{\tau}=\left(\tau_{w} / \rho_{w}\right)^{0.5}$, where, $u_{\tau}$ are is denoted as the friction velocity and $\tau$ is the shear stress. The resultant flow topology is one of multiple generations of wavepackets whereby smaller wavepackets near the wall exist within larger wavepackets residing farther away from the wall, which in turn exist within even larger wavepackets as illustrated in Figure 1-5 (Adrian et al., 2000). The number of generations of wavepackets is determined by the friction Reynolds number of the flow, $\operatorname{Re}_{\tau} \approx \delta / \delta_{v}$, which 
compares the scale of the larger coherent structures, $\delta$, to that of the smaller near-wall structures, $\delta_{v}=v_{w} / u_{\tau}$. Interacting wavepackets are typically neither in perfect spanwise alignment nor contain the same number of hairpin vortices. Thus, merged wavepackets tend to contain fewer hairpin vortices than the younger generation wavepackets (Tomkins \& Adrian, 2003; Adrian, 2007). As the number of incidences of vortex interactions increases, the population of fragmented vortices in the boundary layer also increases, thus making visualization of coherent motions more difficult with increasing Reynolds number (Adrian et al., 2007; Jimenez et al., 2010). However, in close vicinity of the wall where the population of fragmented vortices is relatively smaller, a readily and consistently observed feature in low-, moderate-, and high-Reynolds-number flows is the spanwise-alternating regions of streamwise-elongated low- and high-speed streaks induced by first generation of wavepackets (Marusic et al., 2010). The mean spanwise spacing of the hairpin vortices and their associated low-speed streaks of $\Delta z^{+} \approx 100 \pm 20$

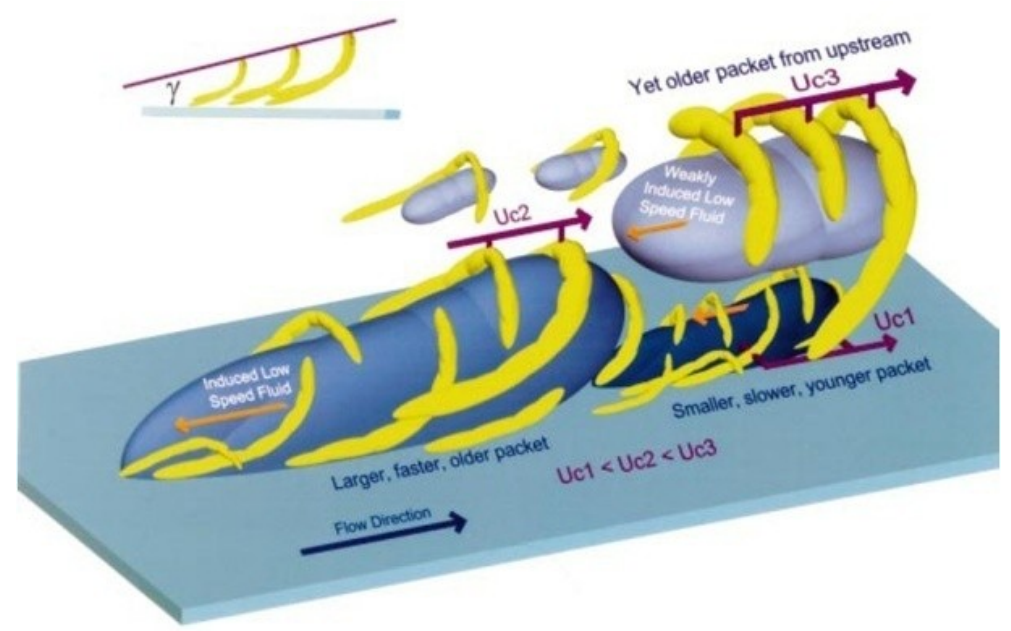

Figure 1-5: Schematic of nested hairpin vortex packets growing up from the wall (Adrian et al., 2000). 
corresponds to the wavelength of perturbations that yields the highest growth rate in the kinetic energy of the perturbations over a period not greater than the local eddy-turnover time, and represents a balance of viscous dissipation and the kinetic energy of the transient wall-normal motions (Baig \& Chernyshenko, 2004; Buttler \& Farrell, 1993). The cumulative ejection of low-speed fluid as a wavepacket convects in the streamwise direction creates the low-speed streaks having lengths ranging from 400 to 1500 wall units depending on the number of vortices in the wavepacket (Brinkerhoff \& Yaras, 2015; Panton, 2001; Smits \& Delo, 2001; Adrian et al 2000; Zhao et al., 1999). The propensity of a hairpin vortex to spawn new vortices, and the frequency at which it does so, is determined by the local shear conditions and disturbance amplitudes that gave rise to its formation (Smits \& Delo, 2001; Adrian et al 2000; Zhao et al., 1999; Tomkins \& Adrian, 2003, Schlatter et al., 2009). As previously stated, the vortices of oldergeneration wavepackets residing farther away from the wall convect faster in the streamwise direction and may overtake their near-wall younger counterparts, yielding another avenue for vortex interaction that may involve cut, connect, and pairing processes. Due to the numerous modes of vortex interactions in fully-turbulent shear layers, hairpin vortices with one leg having significantly stronger coherence than the other are predominant (Panton, 2001; Adrian et al., 2000; Smits \& Delo, 2000; Zhou et al., 1999). For brevity, all configurations of the hairpin-like vortical structure (symmetric, asymmetric, horseshoe, lambda, etc.) will be identified herein as a hairpin vortex, and where necessary distinctions will be made. It is worth mentioning that for all configurations of the hairpin vortex, the fundamental vortex dynamics does not appear to 
significantly deviate from that described above (Panton, 2001; Smits \& Delo, 2000; Adrian et al., 2000; Zhou et al., 1999).

The foregoing description of the development of hairpin vortices has not distinguished between turbulent ZPGBL and channel flows. Near the wall, the topology of the hairpin vortices develops similarly in ZPGBL and channel flows, but as the hairpin vortices move farther away from the wall their development begins to differ between these flows (Hoyas \& Jimenez, 2006; Tompkins \& Adrian, 2003). Unlike turbulent channel flows, the flow in ZPGBL is notably intermittent at wall-normal heights above $0.5 \delta$, containing a mix of large-scale regions of rotational and potential flow (Jimenez et al., 2010). The lack of Reynolds stresses in the potential regions leaves pressure fluctuations as the primary means to homogenize the differences in the streamwise velocities between the slower rotational and faster potential regions (Jimenez et al., 2010). The instantaneous strain rates due to these pressure fluctuations are effective in redistributing kinetic energy amongst the various components of turbulence motions, thus inducing comparatively stronger transverse velocity fluctuations while reducing the streamwise velocity fluctuations for the $y>0.5 \delta$ region (Jimenez et al., 2010). Thus, in this outer region, the hairpin vortices are observed to meander in the spanwise direction to a larger extent in ZPGBL flows than in channel flows (Monty et al., 2009). As a consequence, the wavepackets of hairpin vortices are typically observed to break down in ZPGBL flows once they extend beyond $\mathrm{y}^{+} \approx 200$, and for sufficiently high values of Reynolds number, any apparent coherence of such vortices is lost for $y / \delta>0.3$. In contrast, coherence in wavepackets is typically observed at up to $70 \%$ of the channel half-height. As a result, 
the larger-scale vortices in channel flows have streamwise dimensions as large as $\sim 25 \delta$ compared to $\sim 6 \delta$ in boundary-layer flows (Jimenez et al., 2010, Monty et al., 2009), where for channel flow, $\delta$ represents the channel half-height.

\subsection{Computational Methods for Fluid Mechanics and Heat Transfer}

Computational analyses are an alternative to experiments for cases where detailed modelling of thermalhydraulics is sought. The main limitations of computational analyses are typically associated with assumptions inherent in the formulation and discretization of the governing equations, and the time penalty that must be paid to achieve increased levels of fidelity of the computational results. Furthermore, uncertainties may also be introduced into computational analyses through estimates of the flow state at the computational boundaries, and assumptions on the initial state of the flow. Additional uncertainties may be introduced via the finite spacings in the spatial grid mapped into the computational domain and the finite time duration (timestep) used. Notwithstanding, great success in modelling turbulent flows has been demonstrated through computational methods. Three prominent computational methods for modelling complex wall-bounded turbulent flows are: statistical turbulence modelling; Large Eddy Simulation (LES); and Direct Numerical Simulations (DNS) (Moin \& Mahesh, 1998).

\subsubsection{Statistical Modelling of Turbulence}

Statistical modelling of flow turbulence involves manipulation of the governing equations to solve for an ensemble-averaged flow field. Readers less familiar with the subject may refer to turbulence modelling texts (such as that of Wilcox, 2006) for detailed derivations 
and discussions of these models. The Reynolds Averaged Navier Stokes (RANS) equations, which are the most commonly applied statistical turbulence modelling equations for engineering applications, come about by decomposing the velocity field, $u_{i}\left(x_{j}, t\right)$, into a time-averaged component, $\bar{u}_{i}\left(x_{j}, t\right)$, and fluctuation component, $u_{i}^{\prime}\left(x_{j}, t\right)$, as shown in tensor notation in Equation 1-5.

$$
u_{i}\left(x_{j}, t\right)=\bar{u}_{i}\left(x_{j}, t\right)+u_{i}^{\prime}\left(x_{j}, t\right)
$$

Here, $u_{j}$ is the $j$ Cartesian component of velocity; $x_{j}$ is the $j$ component of Cartesian spatial coordinate; and $t$ is the time coordinate. By combining Equation 1-5 with the governing equations for the instantaneous flow field (i.e. Navier-Stokes equations), a set of equations for the components of the mean velocity can be obtained. This set of equations for the mean velocity field is the RANS equations, and recovers the same form as the Navier-Stokes equations, but with additional terms. These additional terms, identified as Reynolds stresses, originate from the fluctuating velocity field, and may be interpreted as stresses imposed on the mean fluid motion by the fluctuating motions. To close the set of equations, the RANS equations require an additional model that evaluates the Reynolds stresses. This additional model is denoted turbulence closure and may be categorized into zero-equation, one-equation, two-equation, and Reynolds stress models (RSM). The zero-, one- and two-equation models incorporate the Boussinesq hypothesis in their formulations, which presumes the turbulent mixing to be locally isotropic, and presumes dominance of the production and dissipation mechanisms in the turbulence energy budgets. Given these assumptions, the Reynolds stresses can be related to the mean strain rate using the eddy viscosity, $v_{t}$, as the proportionality constant as per Equation 1-6. 


$$
-\overline{u_{i} u_{j}}=v_{t}\left(\frac{\partial \overline{u_{i}}}{\partial x_{j}}+\frac{\partial \overline{u_{j}}}{\partial x_{i}}\right)-\frac{2}{3}(K / \rho) \delta_{i j}
$$

Where $K$ is the turbulence kinetic energy (TKE), $K=0.5 \overline{\rho u_{i}^{\prime} u_{i}^{\prime}}$ and $\delta_{i j}$ is Kronecker delta.

In contrast, RSM directly model the transport of each component of Reynolds through algebraic or differential transport equations. Each Reynolds stress transport equation consists of a production, dissipation, and spatial transport terms for which only the production term is closed and the remaining terms have to be modelled. Dissipation is usually modelled with the assumption that the dissipative eddies are small enough that they can be assumed to be isotropic. The diffusion of Reynolds stresses due to interactions between the pressure and strain fields is modelled to transfer energy from the Reynolds shear stress components to the Reynolds normal stress components such that these normal stresses become more isotropic. These models require the empirical calibration of several coefficients.

\subsubsection{Large Eddy Simulation}

The intent of the Large Eddy Simulation (LES) methods is to model (explicitly or implicitly) the smaller-scale motions while directly computing the larger-scale motions that are more affected by the geometry of the wall boundaries that defines the flow path (Tanehill et al., 1997). This is implemented by applying a low-pass spatial and temporal filter to the Navier-Stokes equations. The model applied to the filtered scales is referred to as the sub-grid scale (SGS) model. The LES method is founded on hypothesis that turbulent flows contain a spectrum of eddies of varying length and velocity scales, and that below certain threshold length and velocity scales the larger-scale geometrical 
features that define the flow path have negligible effect on the development of the eddies. Eddies with scales below these threshold scales are expected to be primarily dissipative, thus, the primary function of SGS model is to provide the correct rate of dissipation. It then follows that the development of eddies confined by these threshold scales may be characterized via a universal model (Stanislas, 2008). This hypothesis is in-line with Kolmogorov's work published in Russian in 1941 and later translated to English (Kolmogorov, 1991), where he demonstrated that the smallest eddies are primarily dissipative, and scale on the kinematic viscosity and rate of dissipation of TKE as per Equation 1-7.

$$
\begin{gathered}
\eta=\left(v^{3} \varepsilon^{-1}\right)^{0.25} \\
u_{\eta}=(v \varepsilon)^{0.25}
\end{gathered}
$$

Where $\eta$ is the Kolmogorov length scale, $\varepsilon$ is the specific rate of dissipation of turbulence kinetic energy, and $u_{\eta}$ is the Kolmogorov velocity scale. In wall-bounded turbulent flows, the minimum value of $\eta$ typically occurs at the wall and is estimated by Equation 1-9 (Stanislas et al 2008).

$$
\begin{gathered}
\eta_{w}=2 v / u_{\tau}=2 \delta_{v} \\
u_{\tau}=\left(\tau_{w} / \rho\right)^{0.5}
\end{gathered}
$$

\subsubsection{Direct Numerical Simulation}

Direct Numerical Simulations (DNS) involve numerically solving the governing equations without the use of turbulence closure. For this technique to be accurate, the spatial and temporal computational grids must be sufficiently fine to capture the smallest spatial and temporal scales relevant to the flow dynamics. These scales are in the order of 
$\eta$ and Kolmogorov time scale, $t_{\eta}=\eta / u_{\eta}=(v / \varepsilon)^{0.5}$ (Moin \& Mahesh, 1998). The spatial and temporal extents of the computation must also be sufficiently large to capture the largest energy containing scales. For example, for ducted flows, the largest scales are in the order of $x \sim 25 \delta, y \sim 2 \delta, \mathrm{z} \sim \delta$, and $t \sim 15 \rho \delta /\langle u\rangle$, in the streamwise, wall-normal, spanwise, and temporal coordinates, respectively (Chin et al. 2010; Tompkins \& Adrian 2003; Elsinga $\&$ Marusic, 2010). Here $\delta$ is taken as one half of the hydraulic diameter and the angled brackets indicate a value that is time and area-averaged over the spanwise/wall-normal plane. The ratio of the largest to smallest scales increases with $\operatorname{Re}^{2.9}$ (Pope, 2000), thus DNS is computationally expensive for simulations of flows of moderate to high Reynolds number. 


\section{Chapter: Introduction and Literature Review}

\subsection{Motivation}

The Generation-IV International Forum (GIF) is an international endeavour that identified six nuclear energy systems with the potential for enhanced safety, efficiency, reliability, and proliferation resistance in comparison to current generation-III nuclear energy systems (GIF, 2009). The potential for attaining improved thermal efficiency in a Rankine cycle though notable increase in the temperature of the heat source provides rationale for further investigation of the SuperCritical Water-cooled Reactor (SCWR) concept, which is one of the nuclear energy systems identified by the GIF. As with other water-cooled nuclear reactors, thermal energy is transferred from the nuclear fuel to the primary coolant as it flows through the fuel channels, where the primary coolant for the SCWR is water at the supercritical thermodynamic state. For pure substances, this thermodynamic state is attained with sufficient increase in fluid temperature and pressure. Canada is invested in the development of the SCWR concept for which thermalhydraulics and safety, materials and chemistry, and design and integration were identified as key areas for fundamental research (Khartabil \& Leung, 2009). The work reported in this thesis is driven by the research requirements for thermalhydraulics (hydrodynamics and heat transfer) of the primary coolant which is at the supercritical thermodynamic state.

Extensive literature surveys on thermalhydraulics experiments for flows of fluids at the supercritical thermodynamic state were presented by Duffey \& Pioro (2005), Pioro \& Duffey (2005), and Pioro ${ }^{\mathrm{a}}$ et al., (2004). Such fluids are hereafter referred to as 
supercritical fluids. These experiments revealed that under certain conditions the heat transfer characteristics of heated flows of supercritical fluids deviated significantly from that of subcritical fluids (e.g. Swenson et al., 1965; Yamagata et al., 1972; Fewster, 1976). Understanding and categorizing the mechanisms that yield such deviation in heat transfer characteristics is the motivation for the present study.

\subsection{Literature Review}

\subsubsection{Forced Convection Heat Transfer to Flows of Supercritical Fluids}

Heat transfer to the coolant flowing through the fuel channels of the SCWR is expected to fall under the turbulent forced convection mode, based on correlations calibrated using subcritical single-phase fluids as per Figure 2-1 (Hofmeister et al., 2007, Metaias \& Eckert, 1968). In this figure, the Rayleigh number, $R a$, quantifies the degree to which buoyancy affects the flow, and is defined as:

$$
R a=\frac{g \beta\left(T_{w}-T_{b}\right) L_{r e f}^{3}}{v \alpha}
$$

where $g$ is the rate of gravitational acceleration, $\beta$ is the volumetric expansion coefficient, $T$ is the static temperature, $L$ is a length scale, $v$ is the momentum diffusivity (kinematic viscosity), $\alpha$ is the thermal diffusivity, and the subscripts $w$ and $b$ refer to wall and bulkfluid conditions, respectively. Excellent surveys of heat transfer experiments performed with ducted flows of various supercritical working fluids were performed by Pioro and co-workers (Duffy \& Pioro, 2005, Pioro \& Duffy, 2005; Pioro $^{\mathrm{b}}$ et al. 2004). The experimental studies in these reviews and additional studies occurring later than or considered outside the scope of the reviews, are listed in Section 2.2.5 for tubular flows 
with upstream-, downstream-, and wall-normal-oriented gravitational acceleration. Such studies have indicated that near the pseudo-critical state, denoted by the subscript $p c$, the heat transfer characteristics for flows of supercritical fluids significantly deviate from established patterns for heated flows of single-phase subcritical fluids as per Figure 2-1. Figure 2-2 depicts the ratio of measured heat transfer coefficients $(H)$ to those calculated via the Dittus-Boelter correlation as per Equation 1-4, at a static pressure of $p=25 \mathrm{MPa}$,

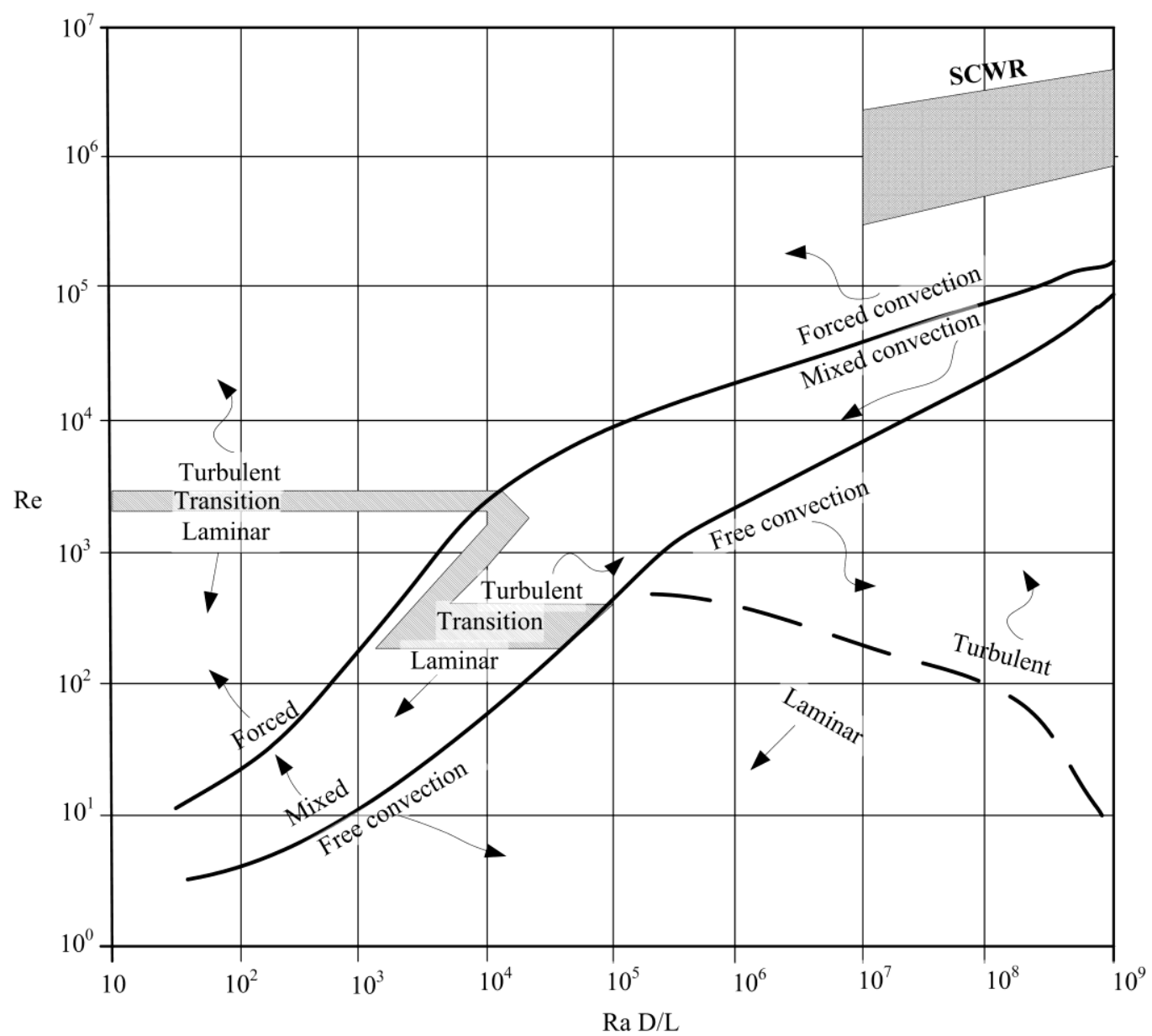

Figure 2-1: Convection heat transfer modes for ducted flows of single-phase subcritical fluid (Metaias \& Eckert, 1968); proposed SCWR operating conditions highlighted (Hofmeister et al., 2007). 


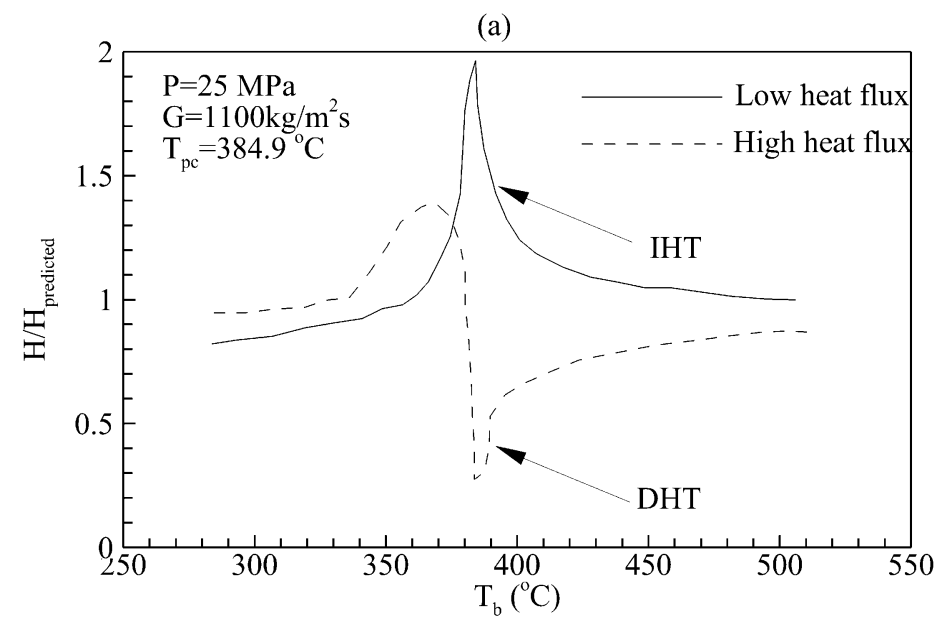

Figure 2-2: Ratio of measured heat transfer coefficient to heat transfer coefficient calculated based the Dittus-Boelter correlation with water as the working fluid (Cheng \& Schulenberg, 2001).

and mass flux of $G=1100 \mathrm{~kg} / \mathrm{m}^{2}$ s with water as the working fluid. Regions where $H$ is either significantly over- or under-predicted by the Dittus-Boelter correlation are evident, and are respectively denoted deteriorated heat transfer (DHT) or improved heat transfer (IHT). Over the past two decades, heat-transfer research with supercritical fluids has focused on predicting the conditions that are conducive to phenomena that lead to heat transfer variations (e.g. Jackson et al., 2011; McEligot \& Jackson, 2004; Hall \& Jackson, 1969), and the magnitude of such variations (Jackson, 2011; Mokry et al., 2011). However, the onset of DHT and IHT is not accurately predicted over ranges of conditions with different fluids, and the heat-transfer models do not accurately predict the $H$ values. Deviations in heat transfer characteristics near the pseudo-critical state may stem from the modified state of turbulence driven by buoyancy and inertia phenomena that are imposed by spatial gradients of thermophysical properties (McEligot \& Jackson, 2004). 
The limited direct numerical simulations (DNS) preformed on supercritical flows support this hypothesis (Bae et al., 2008, 2005).

Correlations to predict the Nusselt number $(\mathrm{Nu})$, which is a non-dimensional heat transfer coefficient, have been proposed for heat transfer in ducted flows of supercritical fluids based on semi-empirical methods. Majority of these correlations modify the empirically derived function " $F$ " in Equation 1-4 (Pioro ${ }^{\mathrm{b}}$ et al., 2004). Other common modifications involve calculating the Reynolds number (Re) and Prandtl number (Pr) using thermophysical fluid properties evaluated at the heated-wall temperature or an average temperature between the wall and the bulk-fluid. However, these correlations remain inadequate under DHT conditions (Mokry et al., 2011; Pioro et al., 2004,).

Despite the large body of experimental data available, the semi-empirical methods discussed in the foregoing have not demonstrated a wide range of success. Among these bodies of work, there is a paucity of experimental research with sufficiently refined spatial or temporal resolution to capture the turbulence characteristics of heated ducted flows of supercritical fluids. Such data could facilitate a more in-depth analysis of the fundamental mechanisms driving DHT and IHT. The paucity of such data is largely due to limitations of measurement techniques under the elevated pressures, temperatures, and corrosive environments associated with the supercritical state. Detailed flow measurements in a vertically-oriented circular duct with carbon dioxide, $\mathrm{CO}_{2}$, as the working fluid were obtained by Kurganov \& Kaptilnyi (1993) at comparable Reynolds numbers to that of the SCWR (see Figure 2-1). The inflow pressure was $p / p_{c}=1.22$, 
which is somewhat higher than that expected for the $\operatorname{SCWR}\left(p / p_{c}=1.13\right)$, where the subscript $c$ indicates the thermodynamic critical state. This higher non-dimensional pressure yields a flow environment with comparatively milder spatial-gradients of the fluid properties. In their experiment setup, total and static pressure measurements were obtained with a pitot tube and axially distributed static pressure taps, respectively. Temperature measurements were obtained with Type-K thermocouples mounted near the $0.1 \mathrm{~mm}$ opening of the pitot tube. Flow velocities were derived from the total and static pressure measurements. These authors noted particular difficulties in obtaining reliable pressure and temperature measurements in close vicinity to the wall, especially in regions experiencing DHT. Accordingly, they reported up to $40 \%$ uncertainty for their temperature and velocity data. In flows with upstream-oriented gravitational acceleration, they observed an "M"-shaped wall-normal profile of the time-averaged streamwise component of velocity, $\bar{u}$, at locations just downstream of DHT as shown in Figure 2-3. Here, the overbar refers to averages taken along statistically-homogeneous coordinates and $y$ is the wall-normal coordinate. This "M"-shaped velocity profile was attributed to inertial/buoyancy-induced near-wall acceleration effects from the local decrease in density and dynamic viscosity as the near-wall temperature increases due to wall heating. In the flow with downstream-oriented gravitational acceleration, the authors noted higher shear stresses near the heated surface compared to subcritical-fluid flows. Another feature of note in this flow was low frequency fluctuations detected in the instantaneous streamwise velocity field near the core of the flow. The authors postulated that these fluctuations indicate the presence of very large scale turbulent flow structures in the 
measured flow region. These flow structures could not be further explored due to limitations on the spatial and temporal resolution of the measured data.

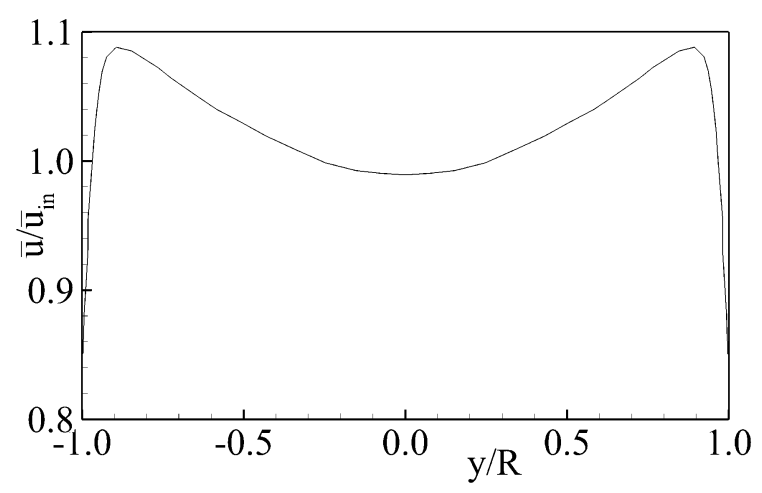

Figure 2-3: Wall-normal distribution of streamwise velocity profile from experimental data of Kurganov \& Kaptilnyi’s (1993).

Computational analyses are an alternative to experiments for cases where detailed modelling of thermalhydraulics is sought. Three prominent computational methods for modelling complex wall-bounded turbulent flows are: Reynolds Averaged Navier-Stokes (RANS) Simulations; Large Eddy Simulation (LES); and Direct Numerical Simulations (DNS) (Moin \& Mahesh, 1998). Application of these methods to heated flows of supercritical fluids is discussed in the subsequent sections with the assumption that the reader has sufficient background knowledge on these methods and on the governing equations of fluid flow, which are respectively discussed in detail in Section 1.4 and Appendix B.1.

\subsubsection{Statistical Turbulence Modelling of Flows of Supercritical Fluids}

Several researchers have studied heat transfer in ducted flows of supercritical fluids using RANS turbulence closures, which include one- and two-equation turbulence closures as 
well as Reynolds stress models (RSM). Limited success has been achieved in modelling heat transfer involving supercritical fluids using zero-, one-, and two-equation turbulence closure (Bazargan \& Mohseni, 2012; Zhang et al. 2011, and $\mathrm{He}^{\mathrm{a}}$ et al. 2008). The works of Bazargan \& Mohseni (2012), Lei et al. (2012), $\mathrm{He}^{\mathrm{b}}$ et al. (2008,) and van der Kraan et al. (2005), are recent RANS efforts in modelling heat transfer in supercritical fluids using the two-equation low-Re $k-\varepsilon$ turbulence closure model. The $k-\varepsilon$ model captures the Reynolds stress by semi-empirically modelling eddies as having a velocity scale proportional to the local turbulence kinetic energy $(\mathrm{K})$ and having a length scale proportional to the local rate of dissipation of $\mathrm{K}(\varepsilon)$. When modelling heat transfer in flows of supercritical fluids, some success has been obtained by re-calibrating the coefficients in the $k-\varepsilon$ formulation of the turbulence closure. However, this success was limited to datasets that were very similar to those used for the semi-empirical recalibration process. Other two-equation closure models, such as the $k-\omega$ model, where $\omega$ is the specific dissipation rate of turbulence, have proven equally unsuccessful as general models for heated flows of supercritical fluid (Jaromin \& Anglart, 2013). The observed limitations of zero-, one-, and two-equation turbulence closures are expected since the Boussinesq hypothesis is inherent in their formulation. This hypothesis presumes the turbulent mixing to be locally isotropic and dominance of the production and dissipation mechanisms in the turbulence budget, which are not supported by the direct numerical simulations of Dave et al. (2013) and Bae et al. (2008) as will be highlighted in Section 2.2.4. 
Turbulence in regions of DHT and IHT is known to be significantly altered through observations of notably reduced turbulent motions under conditions of DHT and vice versa for IHT (Bae et al., 2008). The sharp changes in the topology of the flow turbulence under such conditions indicate that the assumption of dominance of the rates of production and dissipation of turbulence in the zero-, one-, and two-equation models, is violated. These assumptions are abandoned in the formulation of the Reynolds Stress Models (RSM), therefore, more favourable outcomes are expected when RSM models are used for heated flows of supercritical fluids. Indeed, Zhang et al. (2011) confirm this to be the case through comparison of results obtained from a variety of two-equation models and a RSM against published experimental data. Although both the RSM and twoequation turbulence closure were deemed less than adequate by the authors, the RSM was observed to notably outperform the two-equation turbulence closure in predicting the location of DHT and magnitude of the wall temperature excursions under DHT conditions. For example, the RSM of Zhang et al. (2011) featured the "M" shaped velocity profile under similar conditions to the experiments of Kurganov \& Kaptilnyi (1993). Further improvement to RSM may be achieved through optimization of the values of the empirical coefficients in these models. However, this is contingent on obtaining datasets with sufficient detail to facilitate extraction of the relevant turbulence quantities in flows of supercritical fluids. Such datasets are currently not available.

Heated flows of supercritical fluids feature notable regions of DHT and IHT where local buoyancy and inertial forces become non-negligible due to gradients in the thermophysical properties. The RANS equations are derived with the assumption that 
temporal and spatial variations in the density and dynamic viscosity remain insignificant, which is therefore not representative of heated flows of supercritical fluids (Favre, 1965). Versions of the turbulence closure that include a buoyancy production term have been derived, for example by Rodi (1980). In contrast, the contributions of fluctuating viscosity to the turbulence transport equations have been largely neglected. To incorporate the effects of variations of these thermophysical properties, compressible RANS or the Favre-Averaged Navier Stokes (FANS) equations may be solved as an alternative. The FANS equations are similar in derivation to the RANS equations (Section 1.4.1) with the exception that the density-weighted time-averaged velocity $\widetilde{u_{i}}$ replaces the time-averaged velocity as indicated in Einstein notation in the following equation.

$$
u_{i}\left(x_{j}, t\right)=\widetilde{u_{i}}\left(x_{j}, t\right)+u_{i}^{\prime \prime}\left(x_{j}, t\right)
$$

Here, $x_{\mathrm{j}}$ is the $j$ component of Cartesian spatial coordinate, $u_{\mathrm{i}}$ is the $i$ Cartesian component of velocity, $t$ is the time coordinate, the $\sim$ superscript indicates the density weighted timeaveraged value, and the " superscript is used to indicate fluctuation components based on this averaged value. An exception to this denotation is applied to the heat transfer parameter, $Q$, where the " superscript indicates an area flux. To the author's knowledge, FANS simulations have not been performed for flows of supercritical fluids, which is likely due to the scarcity of well-established FANS-based turbulence closures.

\subsubsection{Large-Eddy Simulation of Flows of Supercritical Fluids}

Very few LES studies have been conducted for heated flows of supercritical fluids. Niceno \& Sharabi (2013) performed LES on flows of a supercritical fluid with upstream and downstream-oriented gravitational acceleration using a methodology that applied the 
Wall-Adapting Local Eddy (WALE) viscosity sub-grid model for flow length scales less than $\sim 12 \eta$, where $\eta$ represents the Kolmogorov length scale. Their simulation conditions were selected to coincide with the conditions of the experimental data of Pis'menny et al. (2006). They observed better performance of the LES in predicting DHT trends in comparison to RANS simulations of comparable flow conditions. Figure 2-4 shows their results for conditions conducive to DHT. The time-mean streamwise temperature distribution at the wall agreed fairly well with experimental data. The streamwise location $(x)$ of the onset of DHT was predicted to occur $x / d_{h} \approx 10$ downstream of the measured location, and the streamwise range of locations undergoing DHT was slightly under-predicted. However, the magnitude of temperature excursion at the wall in regions

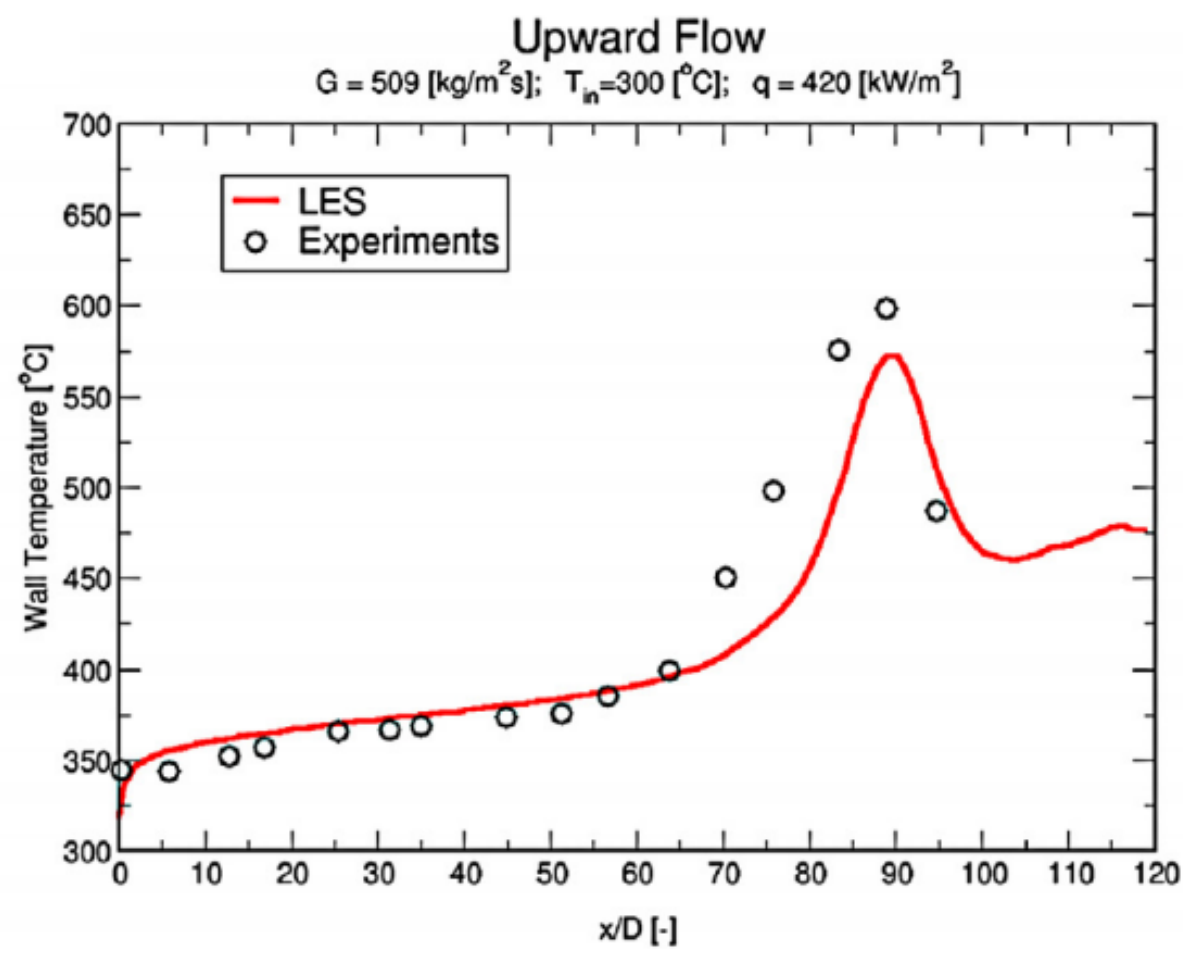

Figure 2-4: Comparison of LES results (Niceno \& Sharabi, 2013) with experimental data (Pis'menny et al. 2006). 
undergoing DHT agreed favourably with experimental data. The notably good performance of this particular LES may be in part due to its well refined spatial grid resolution. It is noted that majority of the most intense dissipative eddies have a spatial scale that ranges from $6 \eta$ to $15 \eta$ (Tanahshi et al., 2004; Moin \& Mahseh, 1998). Accordingly, the spatial grid resolution of $\sim 12 \eta$ employed by Niceno \& Sharabi (2013) is comparable to those of DNS studies. Niceno \& Sharabi (2013) observed an "M"-shape profile of $\bar{u}$ in like manner to the experiments of Kurganov \& Kaptilnyi (1993) in regions where DHT was observed. They noted localized regions where the rate of turbulence production due to the buoyancy force exceeded that due to the shear force and suggested that this is linked to the resultant "M"-shape profile of $\bar{u}$. However, the physical mechanisms leading to such flow physics was unaddressed.

\subsubsection{Direct Numerical Simulation of Flows of Supercritical Fluids}

DNS of heated pipe flows of supercritical $\mathrm{CO}_{2}$ in flow configurations of upstream- and downstream-oriented gravitational acceleration was performed by Bae et al. (2005), and likewise for an annular pipe geometry by Bae et al. (2008) using identical numerical schemes. These simulations were reviewed by Yoo (2013). The findings in these studies were sufficiently similar that the following discussion is apt to both studies. To ease the computational requirements of their DNS, Bae et al. (2005) elected to omit certain terms from the energy-conservation equation. These omissions effectively neglect the conversion of mechanical energy to internal energy by viscous stresses, and neglect the contributions of changes in the local static pressure on the local internal energy of the fluid. These assumptions are commonly applied to low-Mach-number subcritical-fluid flows (Lele, 1994), but their validity for supercritical-fluid flows remains 
unsubstantiated. The simulations were performed for $\operatorname{Re}_{\delta}=5,900$, which is two orders of magnitude lower than that of the experiments of Kurganov \& Kaptilnyi (1993). Where $R e_{\delta}$ is the Reynolds number computed with $\delta$ as the characteristic length scale. It is noted that this reduced $R e$ value causes their simulations to correspond to mixed convection regimes as per Figure 2-1 (Metaias \& Eckert, 1964). Bae et al.'s (2005) computations with upstream-oriented gravitational acceleration featured the "M"-shaped profile of $\bar{u}$ that was also observed in the experiments of Kurganov \& Kaptilnyi (1993). Bae et al. (2005) focused their efforts on analyzing the statistical flow fields, particularly the turbulence budgets. Expectedly, they observed that their results were more effectively represented by Favre-averaged parameters. Bae et al. (2005) noticed similar trends to that of the LES study of Niceno \& Sharabi (2013), whereby the dominant turbulence production in upward flow was buoyancy induced production. However, unlike that of the LES of Niceno \& Sharabi (2013), Bae et al.'s (2008) downward-flow simulation also featured significant buoyancy-induced production of turbulence. Since these two authors perform their simulations at different $R a$ and $R e$ values, reasons for the observed discrepancy cannot be conclusively stated. In Bae et al.'s (2005) simulation, deteriorated heat transfer was suggested to be driven by the relative contributions of the rate of buoyancy-induced production of turbulence $\left(-g \overline{\rho^{\prime} u^{\prime}}\right)$ and that of the mean-shear induced production of turbulence $\left(-\rho u^{\prime \prime} v^{\prime \prime} \partial \tilde{u} / \partial x\right)$ to the overall turbulence production rate. Here, $v$ represents the wall-normal component of velocity. Of note, the so-called buoyancy production term $\left(-g \overline{\rho^{\prime} u^{\prime}}\right)$ may be positive or negative and therefore may enhance or dissipate turbulence. Bae et al. (2005) note that the buoyancy-induced production of turbulence supplemented shear production of turbulence in flows with 
downstream-oriented gravitational acceleration. Conversely, in flows with upstreamoriented gravitational acceleration, the buoyancy-induced production counteracted production of K due to shear in the regions where DHT was observed. Downstream of the DHT region, they observed that the region where buoyancy force begins to supplement the shear forces in the production of turbulence was coincident with the appearance of the "M"-shaped wall-normal profile of $\bar{u}$. Therefore, suggesting that the appearance of the "M"-shaped velocity profile results in the recovery of heat transfer from a deteriorated state by means of increased production of turbulent motions.

From a physics standpoint, Bae et al. $(2005,2008)$ attributed DHT to a reduction in the strength and number of turbulence flow structures in the near-wall region of the flow. They viewed these flow structures by plotting iso-surfaces of vorticity. However, direct links between the statistical flow field and the instantaneous flow field were not provided. Such a linkage could be used to conjure a physical explanation for the mitigation of the near-wall turbulent flow structures as the heat transfer from the wall assumes a deteriorated state. This would be especially relevant since such flow structures are known to significantly contribute to turbulent hydrodynamic and thermal mixing (Wu \& Moin, 2010; Adrian et al. 2000; Kasagi \& Shikazono, 1995). More recent direct numerical simulations, such as those performed by Nemati et al. $(2015,2016)$ and Peeters et al. (2016), are discussed in detail in Section 5.1.2.

\subsubsection{Summary of Literature Review on Heat Transfer in Supercritical Fluids}

There are knowledge gaps in present understanding of the phenomena that drive DHT and IHT in heated flows of supercritical fluids. One such gap pertains to the physical 
mechanisms that lead to flow and heat transfer phenomena that drive DHT and IHT. Once developed, this understanding could facilitate the development of more successful models for heat transfer in such flows. Direct numerical simulations are noted to be well suited for developing an understanding of the detailed turbulence structure of such flows in scenarios where detailed flow measurements are difficult to obtain.

Table 2-1 to Table 2-4 summarize the experiments done on heated flows of supercritical fluid, with $\mathrm{H}_{2} \mathrm{O}, \mathrm{CO}_{2}, \mathrm{He}$, and R134a working fluids. Table 2-5 lists computational efforts geared towards modelling heated-supercritical fluid flows. 
Table 2-1: Selected experimental heat transfer studies with supercritical water flowing in tubes.

\begin{tabular}{|c|c|c|c|c|}
\hline $\begin{array}{l}\text { Author } \\
\text { (Source, if different) }\end{array}$ & $\begin{array}{l}\text { Gravitational } \\
\text { acceleration }\end{array}$ & $\begin{array}{l}d \\
(\mathrm{~mm})\end{array}$ & $\begin{array}{l}\mathrm{G} \\
\left(\mathrm{kg} / \mathrm{m}^{2} \mathrm{~s}\right)\end{array}$ & $\begin{array}{l}\dot{Q}_{W}^{\prime \prime} \\
\left(\mathrm{kW} / \mathrm{m}^{2}\right)\end{array}$ \\
\hline Ackermann (1970) & Upstream & $\begin{array}{l}9.5 \text { to } \\
24.4\end{array}$ & 406 to 1220 & 157 to 1260 \\
\hline $\begin{array}{l}\text { Alekseev et al. (1976) } \\
\text { (Pioro \& Duffey, 2005) }\end{array}$ & Upstream & 10.4 & 380 & 350 to 470 \\
\hline $\begin{array}{l}\text { Domin (1963) } \\
\text { (Hall et al. 1967) }\end{array}$ & Wall-normal & 2 to 5 & 723 to 1010 & 725 to 910 \\
\hline $\begin{array}{l}\text { Herkerath }(1970) \\
\text { Krau et al. }(2008)\end{array}$ & Upstream & 10 & 1500 & 600 to 1000 \\
\hline $\begin{array}{l}\text { Kirillov et al. (1986) } \\
\text { (Pioro \& Duffey, 2005) }\end{array}$ & Upstream & 10.0 & 203 to 1503 & 141 to 726 \\
\hline $\begin{array}{l}\text { Krasyakova et al. (1977) } \\
\text { (Pioro \& Duffey, 2005) }\end{array}$ & Downstream & 20.0 & 1000 & 460 to 1160 \\
\hline $\begin{array}{l}\text { Lee \& Haller (1974) } \\
\text { (Pioro \& Duffey, 2005) }\end{array}$ & Upstream & 38.1 & 1672 & 252 to 1101 \\
\hline Shitsman (1963) & Upstream & 8 & 430 & 280 to 340 \\
\hline $\begin{array}{l}\text { Schmidt (1959) } \\
\text { (Hall et al. 1967) }\end{array}$ & Wall-normal & 5 & 610 & 820 to 910 \\
\hline $\begin{array}{l}\text { Vikhrev et al. (1967) } \\
\text { (Pioro \& Duffey, 2005) }\end{array}$ & Upstream & 20.4 & 495to 1400 & 570 to 1160 \\
\hline $\begin{array}{l}\text { Vikrev \& Lokshin (1964) } \\
\text { (Hall et al. 1967) }\end{array}$ & Wall-normal & 8 & $\begin{array}{l}400 \text { to } \\
1000\end{array}$ & 699 \\
\hline Watts (2009) & $\begin{array}{l}\text { Upstream, } \\
\text { downstream }\end{array}$ & $\begin{array}{l}32.2 \text { to } \\
25.4\end{array}$ & 127 to 617 & 175 to 250 \\
\hline Yamagata et al. (1972) & $\begin{array}{l}\text { Upward, } \\
\text { wall-normal }\end{array}$ & $\begin{array}{l}10.0 \\
7.5\end{array}$ & 1183 to 1830 & 233 to 930 \\
\hline Yu et al. (2013) & $\begin{array}{l}\text { Upward, } \\
\text { wall-normal }\end{array}$ & 26.0 & 600 & 200 to 300 \\
\hline Zhang et al. (2012) & Upward & 10 & 597 to 2021 & 773 \\
\hline
\end{tabular}


Table 2-2: Selected experimental heat transfer studies with supercritical carbon dioxide flowing in tubes.

\begin{tabular}{|c|c|c|c|c|}
\hline $\begin{array}{l}\text { Author } \\
\text { (Source, if different) }\end{array}$ & $\begin{array}{l}\text { Gravitational } \\
\text { acceleration }\end{array}$ & $\begin{array}{l}d \\
(\mathrm{~mm})\end{array}$ & $\begin{array}{l}\mathrm{G} \\
\left(\mathrm{kg} / \mathrm{m}^{2} \mathrm{~s}\right)\end{array}$ & $\begin{array}{l}\dot{Q}_{w}^{\prime \prime} \\
\left(\mathrm{kW} / \mathrm{m}^{2}\right)\end{array}$ \\
\hline Adebiyi \& Hall (1969) & Wall-normal & 22.1 & 200 to 393 & 5 to 26 \\
\hline Bourke et al. (1969) & $\begin{array}{l}\text { Upstream, } \\
\text { Downstream }\end{array}$ & 22.8 & 311 to 1234 & 17 to 270 \\
\hline Bae et al. (2010) & Upstream & $\begin{array}{l}4.4 \\
6.3,9.0\end{array}$ & 400 to 1200 & 29 to 70 \\
\hline $\begin{array}{l}\text { Fewster (1976) } \\
\text { (Duffey \& Pioro, 2005) }\end{array}$ & Upward & $\begin{array}{l}5.0 \\
19.0\end{array}$ & 289 to 636 & 50 to 68 \\
\hline Gupta et al. (2013) & Upward & 8 & 784 to 1000 & 18 to 161 \\
\hline Jiang et al. (2008) & Upward & 0.03 & 432 & 12 to 311 \\
\hline Kim \& Kim (2011) & $\begin{array}{l}\text { Upstream, } \\
\text { downstream }\end{array}$ & 4.5 & 233 to 874 & 53 to 216 \\
\hline Li et al. (2010) & Upward & 2.0 & 315 & 39 to 52 \\
\hline Petukhov et al. (1983) & $\begin{array}{l}\text { Upstream, } \\
\text { downstream, } \\
\text { wall-normal }\end{array}$ & 8 & 3250 & 384 to 1011 \\
\hline Shiralkar \& Griffith (1968) & $\begin{array}{l}\text { Upward, } \\
\text { downward }\end{array}$ & 6.4 & 1012 to 2713 & 138 to 210 \\
\hline Song et al. (2008) & Upward & $4.4,9.0$ & 400 to 1200 & 30 to 50 \\
\hline $\begin{array}{l}\text { Weinberg (1972) } \\
\text { (Duffey \& Pioro, 2005) }\end{array}$ & $\begin{array}{l}\text { Upstream, } \\
\text { downstream }\end{array}$ & 19 & 437 & 30 to 50 \\
\hline Zahlan et al. (2015) & Upstream & 8,22 & 197 to 2027 & 5.1 to 436 \\
\hline
\end{tabular}


Table 2-3: Selected experimental heat transfer studies with supercritical helium flowing in tubes.

\begin{tabular}{|c|c|c|c|c|}
\hline $\begin{array}{l}\text { Author } \\
\text { (Source, if different) }\end{array}$ & Orientation & $\begin{array}{l}d \\
(\mathrm{~mm})\end{array}$ & $\begin{array}{l}\mathrm{G} \\
\left(\mathrm{kg} / \mathrm{m}^{2} \mathrm{~s}\right)\end{array}$ & $\begin{array}{l}\dot{Q}_{w}^{\prime \prime} \\
\left(\mathrm{kW} / \mathrm{m}^{2}\right)\end{array}$ \\
\hline Bogachev et al. (1984) & $\begin{array}{l}\text { Upward, } \\
\text { downward }\end{array}$ & 1.8 & 47 & 0.6 to 1.3 \\
\hline $\begin{array}{l}\text { Brassington \& Carins } \\
\text { (1977) }\end{array}$ & Upward & 18.0 & 48 to 98 & 0.4 to 1.5 \\
\hline Giarratano \& Jones (1975) & Downward & 2.1 & 72 & 0.2 to 4,0 \\
\hline Giarratano et al. (1971) & Upward & 2.1 & 62 to 524 & 1.2 to 8.4 \\
\hline
\end{tabular}

Table 2-4: Selected experimental heat transfer studies with supercritical R134a flowing in tubes.

\begin{tabular}{lllll}
\hline $\begin{array}{l}\text { Author } \\
\text { (Source, if different) }\end{array}$ & Orientation & $\begin{array}{l}d \\
(\mathrm{~mm})\end{array}$ & $\begin{array}{l}\mathrm{G} \\
\left(\mathrm{kg} / \mathrm{m}^{2} \mathrm{~s}\right)\end{array}$ & $\begin{array}{l}\dot{Q}_{w}^{\prime \prime} \\
\left(\mathrm{kW} / \mathrm{m}^{2}\right)\end{array}$ \\
\hline Kang \& Chang (2009) & Upward & 9.4 & 600 & 10 to 60 \\
Zhang (2014) & Upstream & 7.6 & 600 to 2000 & 20 to 100 \\
\hline
\end{tabular}


Table 2-5: Selected numerical studies on heat transfer in ducted flows of supercritical fluids.

\begin{tabular}{|c|c|c|c|c|}
\hline \multirow[t]{2}{*}{ Author } & \multirow{2}{*}{$\begin{array}{l}\text { Numerical scheme } \\
\text { (turbulence models) }\end{array}$} & \multicolumn{3}{|c|}{ Grid resolution (inflow conditions) } \\
\hline & & $\begin{array}{l}\text { Streamwise } \\
\Delta x^{+}\end{array}$ & $\begin{array}{l}\text { Wall-normal } \\
\Delta y^{+} \text {or } \Delta r^{+} \\
\text {(min, max) }\end{array}$ & $\begin{array}{l}\text { Spanwise } \\
\Delta z^{+} \text {or } \\
r \Delta \theta^{+}\end{array}$ \\
\hline Bae et al. (2005) & DNS & 14.5 & $(0.18,5.34)$ & 9.14 \\
\hline Bae et al. (2008) & DNS & 18.9 & $(0.22,-)$ & 7.18 \\
\hline Cheng et al. (2007) & $\begin{array}{l}\text { RANS } \\
(k-\varepsilon \text { and } k-\omega \text { model })\end{array}$ & - & $(1,-)$ & - \\
\hline He et al. (2005) & $\begin{array}{l}\text { RANS } \\
(k-\varepsilon \text { model })\end{array}$ & - & $(0.5,-)$ & - \\
\hline $\mathrm{He}^{\mathrm{b}}$ et al. (2008) & $\begin{array}{l}\text { RANS } \\
(k-\varepsilon \text { model })\end{array}$ & - & $(0.5,-)$ & - \\
\hline $\begin{array}{l}\text { Jaromin \& Anglart } \\
\text { (2013) }\end{array}$ & $\begin{array}{l}\text { RANS } \\
\text { (SST } k-\omega \text { model) }\end{array}$ & $>100$ & $(0.5,-)$ & - \\
\hline Kao et al., (2010) & $\begin{array}{l}\text { RANS } \\
(k-\varepsilon \text { and RSM model) }\end{array}$ & - & $(1.25,-)$ & - \\
\hline $\begin{array}{l}\text { Koshizuka et al. } \\
\text { (1995) }\end{array}$ & $\begin{array}{l}\text { RANS } \\
(k-\varepsilon \text { model })\end{array}$ & - & $(0.1,-)$ & - \\
\hline Li et al (2011) & $\begin{array}{l}\text { RANS } \\
\text { (SST } k-\omega \text { model) }\end{array}$ & - & $(<1,-)$ & - \\
\hline Lei et al (2012) & $\begin{array}{l}\text { RANS } \\
(k-\varepsilon \text { model })\end{array}$ & - & $(0.1,-)$ & - \\
\hline $\begin{array}{l}\text { Mohseni \& } \\
\text { Bazargan, (2012) }\end{array}$ & $\begin{array}{l}\text { RANS } \\
(k-\varepsilon \text { model })\end{array}$ & - & $(-,-)$ & - \\
\hline $\begin{array}{l}\text { Niceno ad Sharabi } \\
\text { (2013) }\end{array}$ & $\begin{array}{l}\text { LES } \\
\text { (WALE model) }\end{array}$ & 70 & $(0.09,20)$ & 9 \\
\hline
\end{tabular}

RSM: Reynolds Stress Model; SST: Shear-Stress Transport; WALE: Wall-Adapting Local Eddy-viscosity 


\section{Chapter: Thesis Objectives}

Although the present research was initiated to support design efforts for the SCWR, its application spans the numerous engineering processes that use heated flows of supercritical fluids. The currently operational supercritical fossil-fuel power plants and the supercritical carbon dioxide power cycle are good examples of such engineering processes. The studies discussed in the preceding chapter indicate that although heat transfer to flows of supercritical fluids is actively studied, the physical mechanisms that drive the unique heat transfer characteristics of such flows are still not well understood. Accordingly, the objectives of the present study are:

1. To develop an understanding of the physical mechanisms through which heat transfer in flows of supercritical fluids deviate from the established physical mechanisms for single-phase flows of subcritical fluids.

2. To develop a set of dynamic similarity criteria that is based on the physical understanding of the flow.

To date, it cannot be conclusively stated whether the phenomena that drive the unique heat transfer characteristics observed in flows of supercritical fluids affect only a limited range of spatial scales of turbulence. Therefore, direct numerical simulations are employed in the present study, where the unfiltered governing equations are solved on a highly refined temporal and spatial grid. 


\section{Chapter: Direct Numerical Simulation of Convective Heat Transfer in a Zero-Pressure-Gradient Boundary Layer with Supercritical Water}

In this Chapter, the development of coherent flow structures in a zero-pressure-gradient boundary layer is investigated in the absence of buoyancy forces, where the working fluid is water in the supercritical thermodynamic state. The aim of the work discussed in this chapter is to confirm the adequacy of the computational method in capturing the unique characteristics of heat transfer in flows of supercritical fluids and highlight deviations observed between these characteristics and those of subcritical single-phase fluids.

Citation: Azih, C., Brinkerhoff, J.R., \& Yaras, M.I., 2012, 'Direct numerical simulation of convective heat transfer in a zero-pressure-gradient boundary-layer with supercritical water', Journal of Thermal Science, 21, pp.49-59.

\subsection{Introduction}

Research attention has recently shifted to understanding the thermal hydraulic behaviour of fluids in the supercritical thermodynamic state, as the lack of phase change and other unique thermodynamic characteristics offer the possibility of improved heat transfer. This new-found interest is fuelled by research and development of the SuperCritical Watercooled Reactor (SCWR) and represents a second wave of interest in the use of supercritical fluids for thermodynamic power cycles. Several heat transfer experiments of turbulent duct flow with supercritical fluids were conducted between the 1950s and 1980s 
for fossil-fuel-based power plants (Pioro \& Duffy, 2004). These early experiments showed that Nusselt number correlations developed for subcritical working fluids can be both under- and over-predictive when the working fluid is in the supercritical thermodynamic state. When the Nusselt number is over-predicted, the heat transfer regime is referred to as "deteriorated heat transfer" (DHT). Conversely, instances where the Nusselt number is under-predicted is referred to as "improved heat transfer" (IHT), and cases in which the Nusselt number is within the accuracy of the subcritical-fluid based predictions is referred to as "normal heat transfer" (NHT) (Pioro ${ }^{b}$ et al., 2004) Although these variations in heat transfer rates were observed decades ago, the underlying physical mechanisms remain poorly understood, casting doubt on the generality of empirical correlations that have been proposed to capture the DHT and IHT cases.

To date, most experimental research has focused on upward-flow in vertical tubes. Correlations for downward-flow in vertical tubes and flow in horizontal tubes are scarce, and to the author's knowledge, no published data exists for boundary-layer flows. The most common empirical methods for characterizing the onset of DHT for flows within vertical tubing give a wide range of predictions in terms of heat-flux to mass flux ratios. From experiments that used water with upward flow, DHT (most likely due to buoyancy phenomena) is observed to occur when $\dot{Q}_{w}^{\prime \prime} / G>1.1 \mathrm{~kJ} / \mathrm{kg}$, where $\dot{Q}_{w}^{\prime \prime}$ is the wall heat flux and $G$ is the mass flux, and IHT occurs at lower values of $\dot{Q}_{w}^{\prime \prime} / G$ (Pioro be al., 2004). In contrast, a fundamental analysis by McEligot \& Jackson (2004) on heat transfer in both 
vertical and horizontal orientations suggests that DHT may occur through a combination of near-wall fluid acceleration, buoyancy effects, and wall-normal fluid-property variation, with the dominant mechanism being determined by the specific flow environment.

In turbulent shear flows, coherent flow structures play a critical role in mass and energy transfer through mixing and hence have a dominant effect on the convective heat transfer coefficient (Buschmann et al. 2009). A study of these coherent flow structures of turbulence via experimental methods has proven difficult due to the elevated pressures and temperatures typically associated with the supercritical thermodynamic state of substances (e.g., for water $T>373.9^{\circ} \mathrm{C}$ and $p>22.06 \mathrm{MPa}$, respectively). Direct numerical simulations (DNS) provide an effective alternative to experimental techniques for the investigation of the role of coherent flow structures in the convective heat-transfer trends observed in supercritical fluids. DNS involves solving the governing equations for fluid flows with a computational grid sufficiently refined to capture all of the spatial and temporal scales of the flow phenomena, such that semi-empirical models are not needed to aid in closure of the governing equations that are filtered in some sense. The present study uses DNS to study the development and interaction of coherent flow structures of turbulence in a zero-pressure-gradient boundary layer with supercritical water as the working fluid under the IHT and NHT conditions. The computational method used to undertake the numerical study and the validation of this computational method are discussed in detail followed by a discussion of the results obtained from the simulations corresponding to the IHT and NHT conditions. 


\subsection{Computational Method}

\subsubsection{Test Cases}

Three direct numerical simulations of the flow development of a zero-pressure-gradient boundary layer on a flat surface are performed. The working fluid in one simulation is air at standard sea-level conditions $\left(F P \_Q\right)$. The results of this simulation are used to validate the numerical approach and to serve as a baseline case for comparing the results of the remaining two simulations that use water above the thermodynamic critical pressure as the working fluid. In the simulations involving supercritical water, the inflow pressure and temperature are $p / p_{c}=1.085$ and $T / T_{p c}=0.968$, respectively, and a wallnormal temperature gradient is imposed on the flow field by means of a spatially-uniform heat flux applied to the test surface. The heat flux is selected to provide an IHT environment (FP_Q0.4) and an NHT environment (FP_Q0.6). Relevant parameters for these test cases are shown in Table 4-1, where $G$ is the spatial average of the mass flux within the boundary layer, and the boundary layer thickness $(\delta)$ is defined by

Table 4-1: Test cases for turbulent flow over a flat surface.

\begin{tabular}{rllrrr}
\hline Case & Working & Thermodynamic & $\dot{Q}_{w}^{\prime \prime} / G$ & $R e_{\tau}$ & $p$ \\
& fluid & state & $(\mathrm{kJ} / \mathrm{kg})$ & $=u_{\tau} \delta_{v} / v$ & $(\mathrm{MPa})$ \\
\hline$F P \_Q 0$ & Air & Subcritical & 0 & 250 & 0 \\
$F P \_Q 0.4$ & Water & Supercritical & 0.4 & 250 & 24 \\
$F P \_Q 0.6$ & Water & Supercritical & 0.6 & 250 & 24 \\
\hline
\end{tabular}


$\bar{u}(\delta)=0.99 u_{\infty}$, where $\bar{u}$ is the time-mean streamwise velocity and $u_{\infty}$ is the free-stream velocity.

All three test cases are simulated to a friction Reynolds number of $R e_{\tau}=250$ which corresponds to a boundary-layer-thickness Reynolds number of $R e_{\delta}=7000$, where $u_{\tau}$ is the friction velocity, and $v$ is the kinematic viscosity at the wall. In the ongoing preliminary design of nuclear reactors utilizing supercritical water to cool the fuel rods, the inlet-flow Reynolds number of water is estimated to be $\operatorname{Re}_{\delta} \sim O\left(10^{5}\right.$ ) (Duffy \& Pioro, 2005). Smith \& Metzler (1983) have shown that the near-wall region of the boundary layer maintains a similar structure for $O\left(10^{3}\right)<R e_{\delta}<O\left(10^{6}\right)$. As forced- convective heat transfer is primarily affected by the flow development in the near-wall region, the similar structure of the near-wall flow over such a large Reynolds number range implies that similar physical mechanisms occur in SCWR as in the present simulations.

\subsubsection{Computational Domain}

The computational domain used to carry out the studies is shown in Figure 4-1 and contains a region of interest in which data is analyzed. To capture all the relevant flow structures in both the inner and outer regions of the boundary layer, the computational domain must be large enough to contain the largest wavelength of turbulence phenomena expected in the flow field. Flow structures with streamwise lengths of up to $20 \delta$ have been observed in the outer part of the boundary layer (Hutchins \& Marusic 2007). 


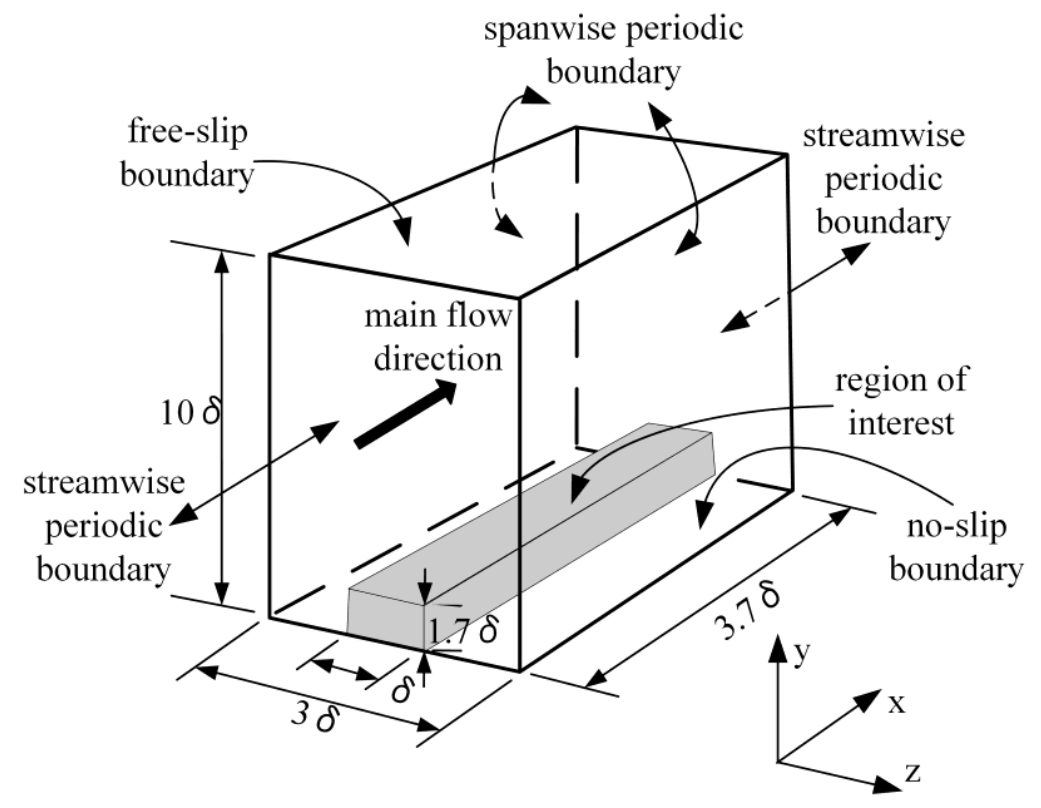

Figure 4-1: Schematic of the computational domain for the turbulent flat-surface cases; $\mathrm{x}$-scale $/ \mathrm{y}$-scale $=2.5, \mathrm{x}$-scale $/ \mathrm{z}$-scale $=1$.

Therefore the streamwise length of the computational domain of $3.7 \delta$ is not sufficient for detailed study of the outer part of the boundary layer. However this streamwise length is shown by Moin \& Mahesh (1998), and Jimenez \& Moin (1991) to be sufficient for analysis of turbulence energy budgets and other turbulence statistics in the inner region of the boundary layer $\left(y^{+}<40\right)$. Here, the + Superscript denotes normalization by the viscous length scale, $\delta_{v}=v / u_{\tau}$, and the friction velocity, $u_{\tau}=\left(\tau_{w} / \rho\right)^{0.5}$, where $\tau_{w}$ is the shear stress at the wall, and $\rho$ is the fluid density; the values of $v$ and $\rho$ in the evaluation of $u_{\tau}$ and $\delta_{v}$ are taken at the wall.

The spanwise extent of the largest coherent flow structures in the boundary layer are of $O(\delta)$, and take the form of streamwise-aligned wave-packets of hairpin-like vortical structures in the outer region of the boundary layer. These structures form through 
spanwise merging of near-wall vortex loops that have a spanwise spacing of $\Delta z^{+} \approx 100$ (Panton, 2001; Osterlund et al., 2003; Tompkins \& Adrian, 2003). To accurately capture the structures in the inner part of the boundary layer, a computational-domain span greater than 220 wall units must be used (Jimenez \& Moin, 1991). Accordingly, the span of the region of interest is sized to 310 wall units. The region of interest in the computational domain has streamwise, spanwise and wall-normal dimensions of $\Delta x=3.7 \delta, \Delta z=\delta$, and $\Delta y=1.7 \delta$, respectively. The wall-normal height is more than sufficient to resolve the instantaneous heights of the coherent flow structures expected in the turbulent boundary layer (White 1991, Pope, 2000). For the test cases involving heat transfer, the Prandtl number remains greater than unity; thus, the thermal boundary layer is contained within the velocity boundary layer, hence remains within the region of interest.

\subsubsection{Boundary and Initial Conditions}

A no-slip boundary condition is applied to the lower wall of the computational domain while zero wall-normal velocity and shear stress are applied to the upper wall. The heat flux values at the no-slip boundary are specified according to the values in Table $4-1$. The flow is developed from a spatially uniform initial condition using streamwise and spanwise periodic boundaries such that the solution variables (pressure, velocities and temperature) are mapped between corresponding nodes on the periodic faces. Since no inflow and outflow boundary conditions are specified, the mass flow rate through the domain is specified through the initial condition. The domain is initialized with a spatially-uniform velocity of $1.6 \mathrm{~m} / \mathrm{s}$ in the subcritical case and $0.013 \mathrm{~m} / \mathrm{s}$ in the supercritical cases. The temperature and pressure fields of the supercritical-fluid test 
cases are initialized with spatially uniform values of $633 \mathrm{~K}$ and $24 \mathrm{MPa}$, respectively. To isolate the heat-transfer variation mechanisms that result from local property gradients from those mechanisms related to buoyant interactions within the flow, no body forces are specified.

As the simulation progresses, the temporal boundary-layer growth matches the Blasius prediction of the laminar boundary layer until transition to turbulence takes place. To ensure that the flow is turbulent at the target Reynolds number, disturbances are introduced into the flow by way of a mass source/sink pair located in close proximity to the no-slip boundary. The mass source disturbance is introduced at a momentumthickness Reynolds number $\left(R e_{\delta_{2}}=u_{\infty} \delta_{2} / \nu_{w}\right)$ of 450 , where the momentum thickness, $\delta_{2}$, is defined as:

$$
\delta_{2}=\int_{0}^{\infty}\left(\frac{\rho \bar{u}(y)}{\rho_{\infty} u_{\infty}}\left(1-\frac{\bar{u}(y)}{u_{\infty}}\right)\right) d y
$$

The source strength is specified through the mass flow rate, and injects fluid into the domain at a wall-normal velocity equal to $10 \%$ of the free-stream velocity. The disturbance, introduced for a duration $\Delta t^{+}=\left(\Delta t \times u_{\tau}^{2}\right) / v=4.5$ for all test cases, is centred between the streamwise and spanwise boundaries.

\subsubsection{Spatial and Temporal Grids}

A structured grid consisting of hexahedral volumes is mapped onto the computational domain shown in Figure 4-1 and consists of a region with high spatial resolution surrounded by a region in which the spatial resolution is gradually coarsened with distance from the finely resolved region. The finely resolved region (labelled as the 
region of interest in Figure 4-1) is populated by 417, 135, and 96 nodes in the streamwise, wall-normal, and spanwise directions, respectively. Outside this region, the node spacing is increased at a constant rate of $12 \%$ until the boundaries of the computational domain are reached. To simulate the cases presented in Table 4-1, the spatial and temporal grids must be sufficiently refined to capture the smallest spatial and temporal scales in the flow, which are on the order of the Kolmogorov length and time scales given by Equation 4-2 and 4-3, respectively.

$$
\begin{gathered}
\eta=\left(v^{3} \varepsilon^{-1}\right)^{0.25} \\
t_{\eta}=\left(\frac{v}{\varepsilon}\right)^{0.5}
\end{gathered}
$$

Where $\varepsilon$ is the rate of turbulence kinetic energy dissipation. For wall-bounded turbulent shear layers, the Kolmogorov length scale is on the order of the viscous length scale, typically reaching a minimum value of $\eta^{+} \approx 2$ close to the no-slip boundary (Kim et al., 1987; Stanislas et al., 2008). Thus, the nodes are distributed such that the average spacing in the region of interest yields values of $\Delta x^{+}=3$ and $\Delta z^{+}=3$, corresponding to approximately 1.5 times the Kolmogorov length scale at the target Reynolds number. At this Reynolds number, the $y^{+}$value of the first node from the no-slip boundary is 0.85 . Close to the wall, $0<y^{+}<30$, the wall-normal node spacing is increased linearly from the value at the wall to a value that corresponds to the Kolmogorov length scale in the loglaw region, which is approximated by:

$$
\eta^{+}=\left(\kappa y^{+}\right)^{0.25}
$$

where $\kappa=0.41$ is the von-Karman constant boundary (Kim et al., 1987; Stanislas et al., 2008). Above the log-law region, the node spacing is kept constant until $1.7 \delta$ is reached, 
after which the node spacing is increased at a rate of $12 \%$ until the free-slip ceiling is reached. The wall-normal node distribution is shown in Figure 4-2.

In the cases with supercritical water as the working fluid, the node spacing is adjusted in the wall-normal direction to capture variations in the thermal field according to:

$$
\Delta y_{\text {thermal }}=\Delta y_{\text {velocity }}(\operatorname{Pr})^{-1 / 3}
$$

where $\Delta y_{\text {thermal }}$ is the node spacing requirement for the thermal boundary layer and $\Delta y_{\text {velocity }}$ is the node spacing requirement for the hydrodynamic boundary layer (RedjemSaad et al., 2007; Kong et al., 2000).

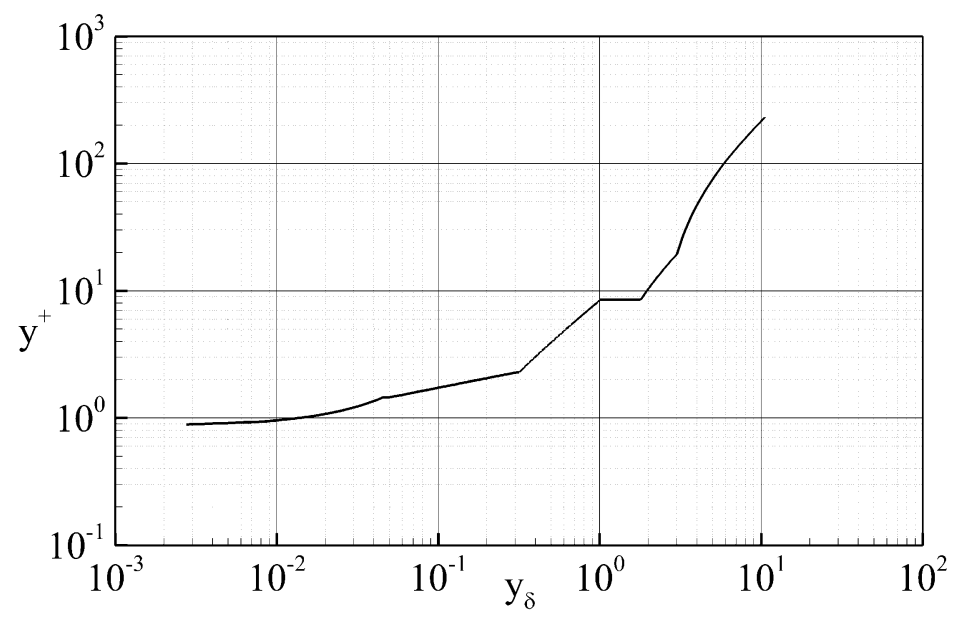

Figure 4-2: Node spacing in the wall-normal direction for the flat-surface cases.

For wall-bounded turbulence, a timestep of $\Delta t^{+}<0.2$, where a value of 0.2 corresponds to a tenth of the Kolmogorov time scale, is required to capture the temporal development of turbulence structures (Friedrich et al., 2001). Besides capturing the flow physics, the temporal resolution must also be selected to ensure numerical stability. In the present study, a constant timestep value of $\Delta t^{+}=0.12$ is used for the subcritical fluid case and a 
value of $\Delta t^{+}=0.013$ is used for the supercritical-fluid test cases to ensure numerical stability.

\subsubsection{Solution Method}

The commercial computational-fluid-dynamics software package ANSYS CFX® (Version 12) is used to perform the simulations in the present study. The solver discretizes the conservation form of the mass, momentum and energy equations (Appendix B.1) using an element-based finite-volume technique, which is documented in the ANSYS CFX-solver theory guide (ANSYS, 2009). The governing equations are discretized using central differencing for the spatial derivatives and second-order backwards Euler scheme for the temporal derivatives. Closure of the governing equations is achieved using the equation of state for the working fluid. The thermodynamic state of air is modelled by the ideal gas law with a constant specific heat value of $C_{p}=1.0044 \mathrm{~kJ} / \mathrm{kg} \mathrm{K}$. The International Association for the Properties of Water and Steam (IAPWS) equations of state are used to model the thermodynamic state of water above the thermodynamic critical pressure (IAPWS, 2008; IAPWS, 2009). The IAPWS formulations are utilized by the solver by means of look-up tables specified through pressure and temperature inputs. To prevent extrapolation of property data, the pressure and temperature ranges utilized in the current study correspond to over four times the expected variations in these properties estimated from similar numerical and experimental studies (Brinkerhoff \& Yaras, 2014; McAuliffe \& Yaras, 2007). The property table is generated with temperature and pressure increments of $\Delta T=0.2 \mathrm{~K}$ and $\Delta p=0.08 \mathrm{MPa}$, which was determined to be suitable to provide values for all relevant 
thermodynamic properties through linear interpolation with an accuracy that is equivalent to the uncertainty associated with the IAPWS equations of state.

Solution convergence of the discretized algebraic equations is obtained using up to eight outer loop iterations per timestep, and residuals are declared as converged when the normalized root-mean-square (rms) residuals (ANSYS, 2009) of the governing equations

are reduced to less than $10^{-5}$. The simulations are run in parallel using 20 Intel ${ }^{\circledR}$ L5410 Xeon ${ }^{\circledR}$ CPUs, and take approximately 11,000 CPU hours (42,000 timesteps) for $F P \_Q 0$, and 26,000 CPU hours $(120,000$ timesteps) for the FP_Q0.4 and FP_Q0.6. The simulation duration, for the $F P \_Q 0, F P \_Q 0.4$, and $F P \_Q 0.6$ is 4800,1625 , and 1300 wall units, respectively.

\subsection{Results and Discussion}

\subsubsection{Validation of Numerical Approach}

Validation of the computational approach for the turbulent flat-surface cases is accomplished by comparing the results of $F P \_Q 0$, which simulates unheated air flow over a flat surface at standard sea-level conditions, to statistical turbulence properties of corresponding turbulent boundary layers from literature. For the turbulent flat-surface cases, the streamwise-periodic boundaries cause the temporal development of the boundary-layer to occur in a spatially-uniform manner. Therefore, the temporal statistics for a given wall-normal location are obtained through a spatial average of 30,000 points in the corresponding wall-normal plane within the region of interest. 
The mean velocity profile, mean turbulence kinetic energy budgets and root-mean-square streamwise velocity fluctuation at the target Reynolds number are presented in Figure 4-3. Figure 4-3(a) shows excellent agreement in the predicted mean velocity profile for $y^{+}<50$ when compared to the DNS of Wu \& Moin (2009), who simulated the ZPGBL without streamwise-periodic boundaries, and used disturbances introduced into the free-stream (rather than near the wall) to trigger transition to turbulent flow. Adequate agreement is also achieved in the outer part of the boundary layer, with a deviation of less than $10 \%$ in the mean velocity profile. The limitation in the accuracy of the outer part of the boundary was mentioned in Section 4.2.2, and is due to the streamwise length of the computational domain being insufficient to fully capture turbulence in the outer region of the boundary layer, where turbulence structures of larger spatial and temporal scales dominate. Figure 4-3(c) shows that in the inner part of the boundary layer, the turbulence kinetic energy production rate $P^{+}$, and dissipation rate $\varepsilon^{+}$are similar to the results of Spalart (1988). In both simulations, the peak $P^{+}$value occurs at $y^{+} \approx 11$ with a value $P^{+}=0.27 \pm 0.01$. Similar agreement is also achieved for the root-mean-square streamwise velocity fluctuation, $u_{r m s}^{\prime+}$, (Shown in Figure 4-3(b)), as well as other statistical turbulence properties. The quantity $u_{r m s}^{+}$is maximum at $y^{+} \approx 13$ which is consistent with the results of Spalart (1988). 

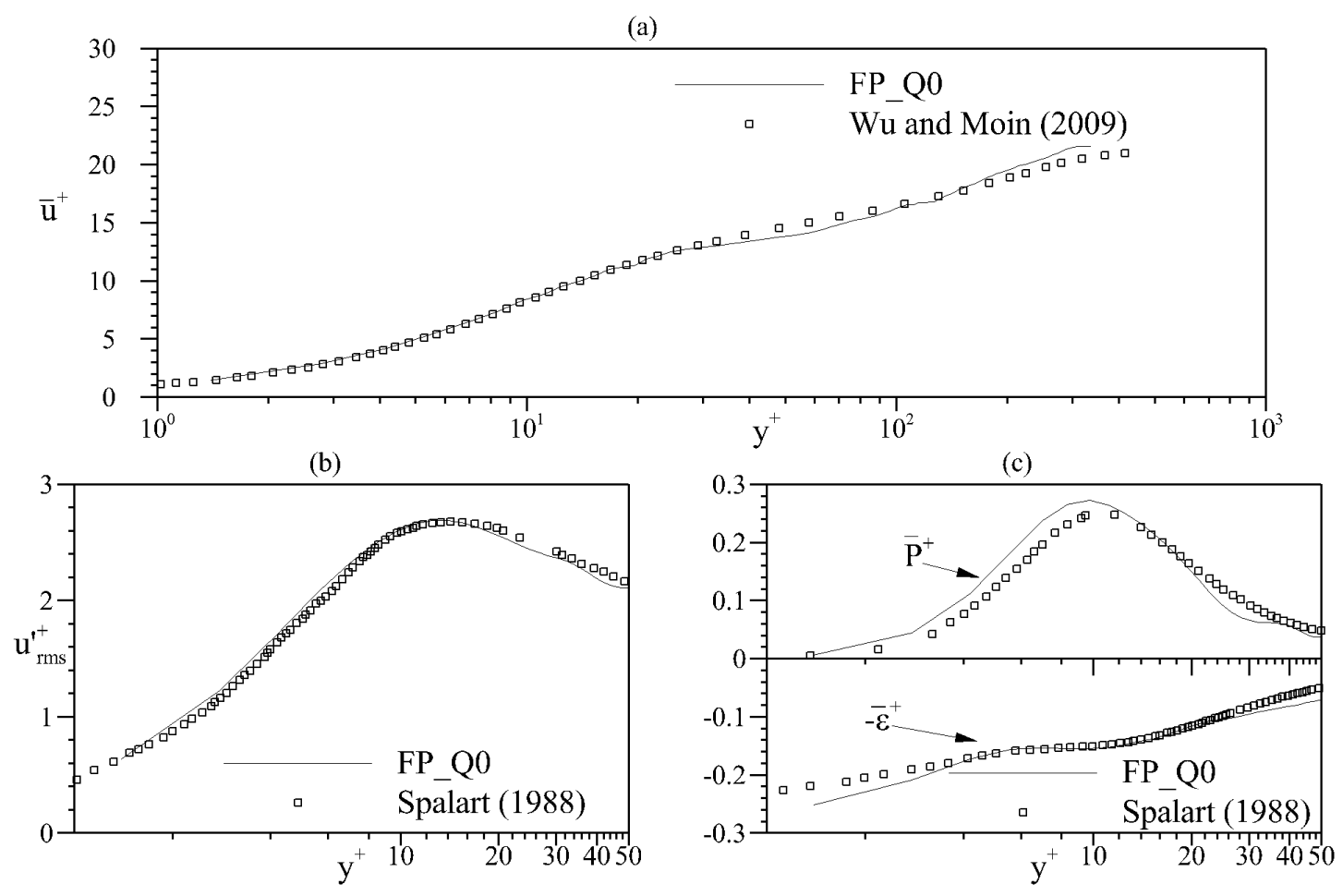

Figure 4-3: (a) Mean velocity profiles; (b) Root-mean-square of the streamwise velocity fluctuation (c) Mean rate of turbulence kinetic energy production and dissipation. FP_QO $\left(R e_{\delta_{2}}=900\right)$, Spalart $\left(R e_{\delta_{2}}=1410\right), \mathrm{Wu} \& \operatorname{Moin}\left(R e_{\delta_{2}}=900\right)$.

As heat transfer is dominated by near-wall flow turbulence activities, it is of critical importance that the near-wall flow structures be accurately resolved by the numerical method (Buschmann et al., 2009). The near-wall region of the boundary layer is dominated by hairpin-like vortical structures and high- and low-speed streaks. The streaks are created by streamwise-oriented vortices, such as the legs of the hairpin-like vortical structures, the induced velocities of which yield a wall-normal momentum exchange and thus regions of momentum excess or deficit (i.e. streaks) [25-27]. These coherent flow structures are visualized in Figure 4-4 through iso-surfaces of the second invariant of the velocity-gradient tensor, $q^{+}=0.01$. These iso-surfaces reveal hairpin-like 


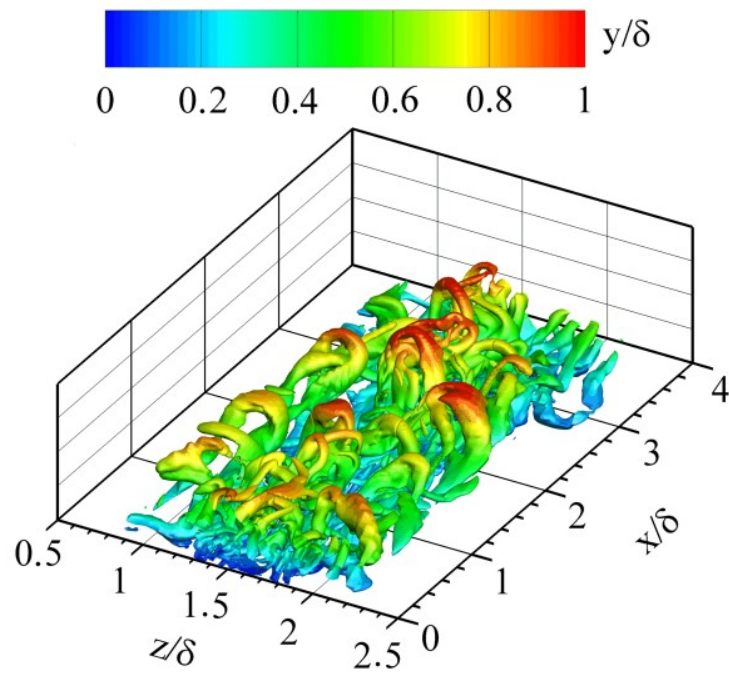

Figure 4-4: Iso-surface of the second invariant of the velocity-gradient tensor, $q^{+}=0.01$, for $F P \_Q 0$, coloured by wall-normal height; $R e_{\delta_{2}}=930$.

vortical structures as the dominant flow structure populating the outer part of the boundary layer, which agrees with the observations in published literature (Wu \& Moin, 2009; Adrian et al., 2000; Head \& Bandyopadhyay, 1981). Colouring the iso-surfaces by $y / \delta$ demonstrates that these coherent structures extend to the edge of the boundary layer. The streamwise spacing of these hairpin-like structures is shown at $R e_{\delta_{2}}=390$ in Figure 4-5 through contours of the spanwise component of vorticity $(\omega)$. The streamwise spacing between individual structures is observed to be 140-180 wall units and the structures reach wall-normal heights of up to 300 wall units, which is consistent with published literature at similar Reynolds numbers (Camussi \& Felice, 2006; Adrian et al., 2000; Zhou et al., 1999). In addition, the spanwise spacing of near-wall streaks has an average value of 103 wall units, obtained via analysis of the power spectral density of the fluctuation component of streamwise velocity $P S D_{u^{\prime}}$. This spacing is consistent with published results of $\Delta z^{+} \approx 100$ (Panton, 2001). The favourable agreement between the 
results of the FP_QO case with results from several published works validates the numerical approach used for the analysis of the ZPGBL, especially in the inner part of the boundary layer $\left(y^{+}<50\right)$.

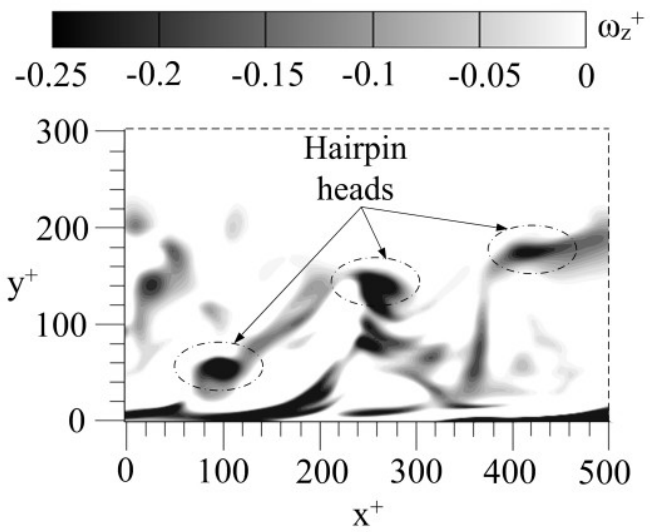

Figure 4-5: Hairpin-like structures viewed via contours of instantaneous z-vorticity at plane $z / \delta=1.5 ; R e_{\delta_{2}}=930$.

\subsubsection{Effect of Supercritical Thermodynamic State on Heat Transfer}

Cases FP_Q0.4 and FP_Q0.6 simulate the flow of water at a static pressure greater than the thermodynamic critical pressure of water with heat flux values of $\dot{Q}_{w}^{\prime \prime} / G=0.42$ and $\dot{Q}_{w}^{\prime \prime} / G=0.60$, respectively specified at the no-slip boundary. The wall heat fluxes and mass flow within the boundary layer in these cases correspond to environments where IHT is predicted at the target Reynolds number (Pioro \& Duffy, 2005). Since the baseline case $\left(F P \_Q\right)$ is not heated, it is of interest to establish the Nusselt number values $(\mathrm{Nu})$ for these conditions that are predicted by semi-empirical correlations "calibrated" for subcritical single-phase thermodynamic states. One such correlation uses the ReynoldsColburn analogy (Equation 4-6) to express $N u$ as a function of Prandtl number $(P r)$ and 
Reynolds number (Re) (Holman, 2002); Another correlation by Churchill (1976) takes into account the Nusselt number as a function of the transition location (Equation 4-7).

$$
\begin{gathered}
N u=0.0308 R e_{x, f}^{4 / 5} \operatorname{Pr}_{f}^{1 / 3} \\
N u=0.4637\left[\varphi+\frac{\varphi^{8 / 5}}{209\left(1+\left(\frac{\varphi_{u}}{\varphi}\right)^{7 / 2}\right)^{2 / 5}}\right]^{1 / 2}+0.45 \\
\varphi=\frac{R e_{x, f} P r_{f}^{2 / 3}}{\left[1+\left(\frac{0.02052}{P r_{f}}\right)^{2 / 3}\right]^{1 / 2}}
\end{gathered}
$$

Here, $\varphi_{u}$ is the value of $\varphi$ evaluated at the streamwise location where transition is completed (Churchill, 1976), and the subscript $f$ indicates that thermophysical property values are evaluated at the film temperature, $T_{f}=\left(T_{w}+T_{b}\right) / 2$. Equation 4-6, and 4-7 respectively predict a $N u$ value of 415 and 450 for the lower wall-heat-flux case and predict 386 and 431 for the higher wall-heat-flux case. These $N u$ values are indicated in Table 4-2.

Table 4-2: Nusselt numbers for the cases with supercritical water as the working fluid.

$$
N u \text { (Equation 4-6) Nu (Equation 4-7) Nu (DNS) }
$$

\begin{tabular}{llcc}
\hline$F P \_Q 0.4$ & 415 & 450 & 533 \\
$F P \_Q 0.6$ & 386 & 431 & 421 \\
\hline
\end{tabular}

In the lower wall-heat-flux case, the $N u$ value is $22 \%$ and $16 \%$ higher than the values predicted by Equation 4-6 and Equation 4-7, respectively. Conversely, in the higher wallheat-flux case, the $N u$ value is $9 \%$ lower and $2 \%$ higher than the values predicted by 
Equation 4-6 and Equation 4-7, respectively. The heat transfer in the former case clearly corresponds to a condition of IHT, and the $9 \%$ difference for the latter case is only slightly greater than the uncertainty of the value predicted by Equation 4-6, which indicates that the effects of mechanisms that drive IHT are being mitigated.

In heated flows of Reynolds numbers similar to the present study, in which the fluid remains in the subcritical thermodynamic state, temperature is observed to act as a passive scalar, i.e. the temperature field does not appear to affect the kinematics of the flow (Kong et al. 2000; Wu \& Moin, 2010). Inspection of a single wave-packet, observed through $z$-vorticity contours as shown in Figure 4-6, reveals strong similarity in the larger structures between the heated cases in the vicinity of $100<y^{+}<300$, both in streamwise and wall-normal spacing. However, closer to the wall $\left(y^{+}<60\right)$ while the contours of $z$ vorticity remain attached to the wall in the lower wall-heat-flux case, the case with the higher wall-heat-flux exhibits a different behaviour wherein discrete regions of $z$-vorticity are observed to break-off from the no-slip surface. As the only difference between these cases is the wall heat flux, this observation suggests that the temperature field has an influence on the kinematics of the flow when the working fluid is in the supercritical thermodynamic state.

As discussed in Section 4.3.1, the near-wall region of the boundary layer is populated by low- and high-speed streamwise streaks. Figure 4-7 plots contours of instantaneous streamwise velocity fluctuation in the near-wall region for the cases with heated walls, 


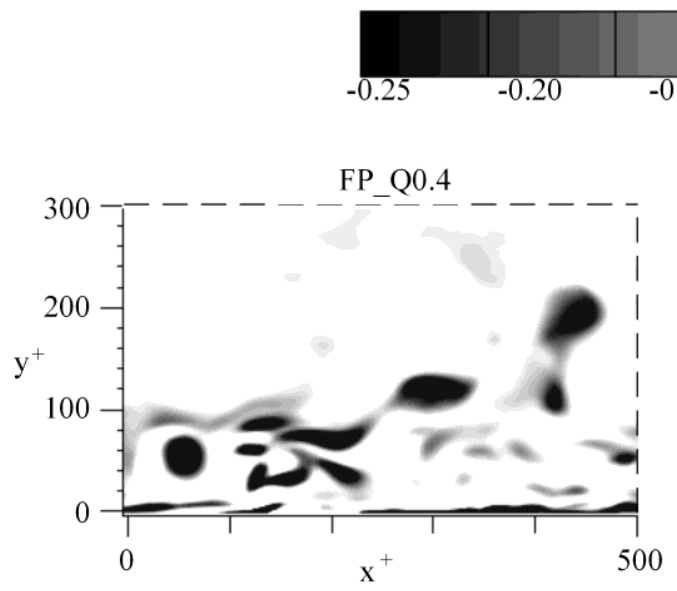

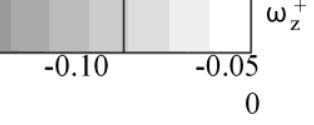

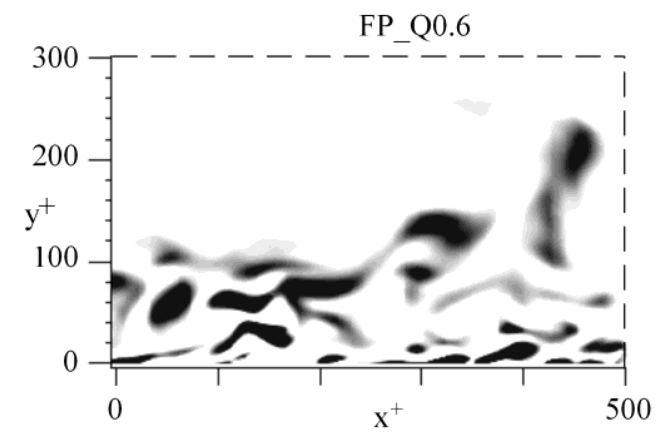

Figure 4-6: Contours of z-vorticity for the heated turbulent flat-surface cases $R e_{\tau}=250$.

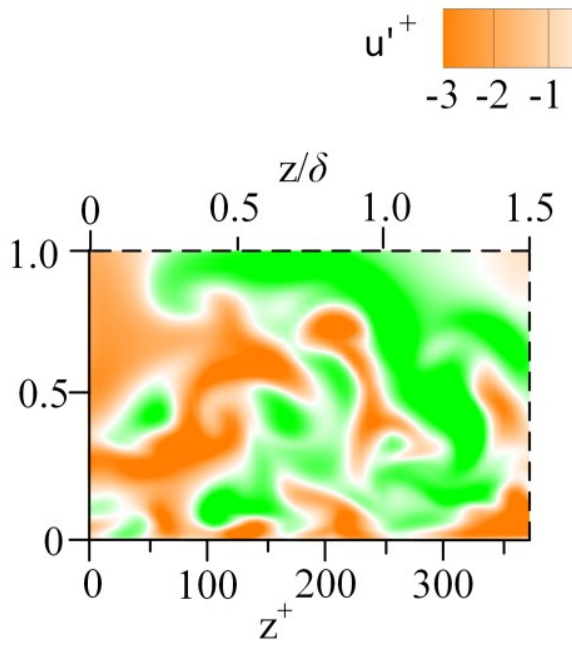

FP_Q0.4

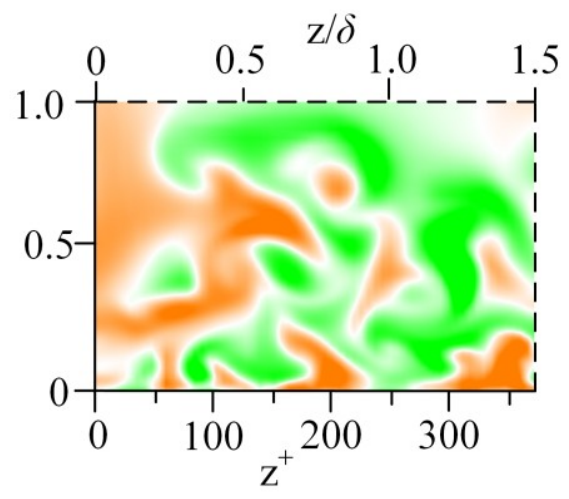

FP_Q0.6

Figure 4-7: Streaks viewed through iso-contours of the instantaneous streamwise perturbation velocity component; turbulent flat-surface cases. $R e_{\tau}=250$.

with a "traffic-light" colour scheme that indicates low-speed streaks with amber colouring and high-speed streaks with green colouring. The plots in Figure 4-7 are generated using data from the same instance in time and spanwise/wall-normal plane from the two heated-wall cases such that the development of the coherent structures of turbulence can be directly contrasted between the two plots. It is observed that in the 
higher-wall heat flux case the near-wall low-speed streaks appear weaker than those of the other case. These streaks are formed by the downwash motion on the outboard sides of hairpin vortices, and this downwash motion enhances thermal mixing by transporting colder fluid towards the heated wall. Thus, it is likely that the mechanisms cause the degradation of the low-speed streaks are also responsible for mitigating the effects of phenomena that lead to IHT. Such changes in the small-scale turbulent mixing may also affect the large-scale coherent flow structures in the outer region of the turbulent boundary layer. This is shown in Figure 4-8 through plots of iso-contours of the second invariant of the velocity-gradient tensor. Hairpin-like structures are observed in the outer boundary layer, although they do not retain much of their coherence in comparison to the baseline case illustrated in Figure 4-4.

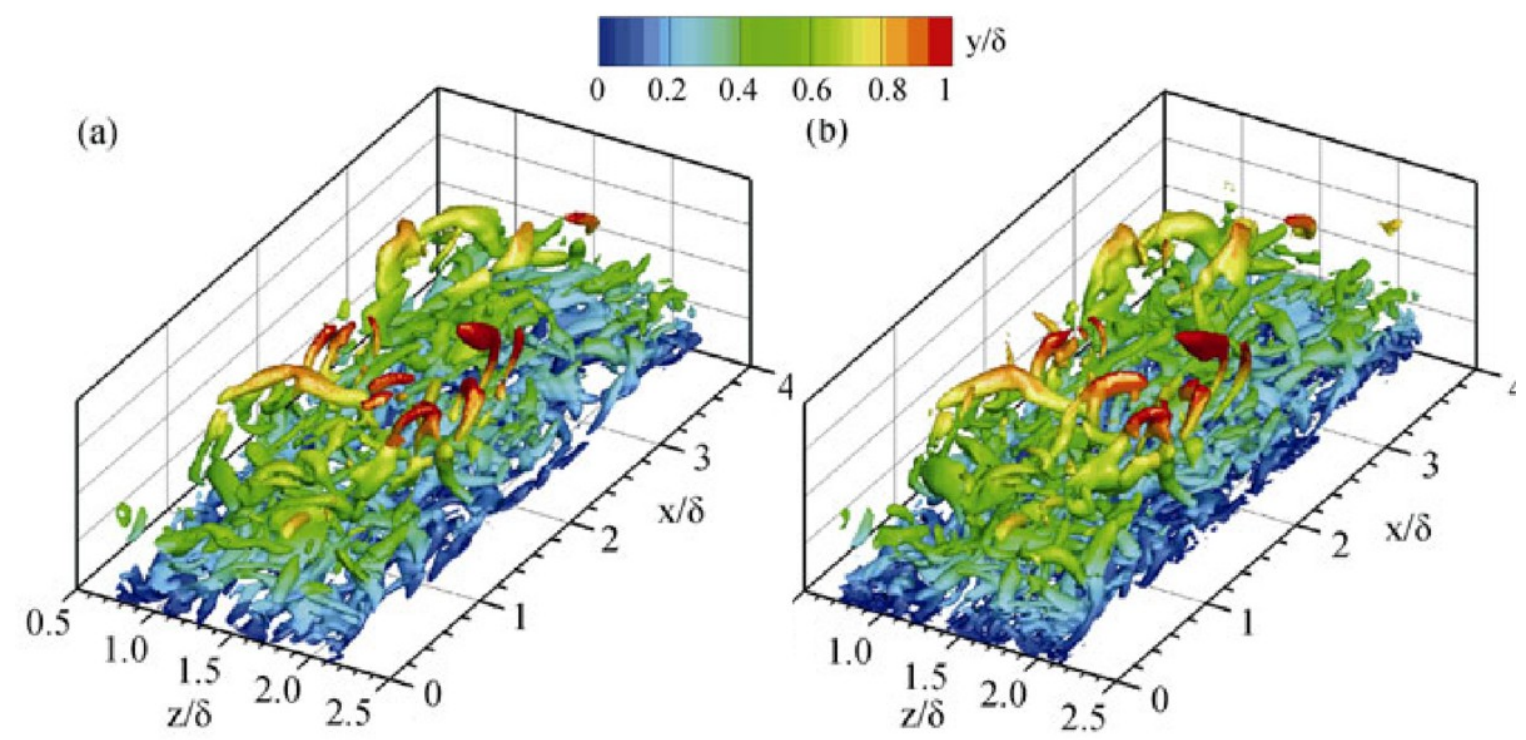

Figure 4-8: Iso-surfaces of the second invariant of the velocity-gradient tensor, $q^{+}=0.01$, coloured by wall-normal height; a) Case $F P \_Q 0.4$, b) Case $F P \_Q 0.6$. 


\section{Chapter: Effects of spatial gradients in thermophysical properties}

\section{on the topology of turbulence in heated channel flow of supercritical}

\section{fluids}

In the previous chapter, it is demonstrated that the development of coherent structures of turbulence may differ in heated flows of fluids at the supercritical thermodynamic state, when compared to single-phase subcritical fluids. These differences may have an effect on the heat transfer rate. A study was performed, documented in Appendix A, to compare the relative contributions of laminar (molecular-diffusion) driven phenomena and turbulence-driven phenomena on heat transfer in flows of supercritical fluid. The study shows the effects of changes in the flow turbulence on the heat transfer rate to be an order of magnitude higher than the laminar-driven effects. Building on the study in

Appendix A, this chapter details the topology of turbulence in heated channel flows of supercritical fluids.

Citation: Azih, C. \& Yaras, M.I., 2018, 'Effects of spatial gradients in thermophysical properties on the topology of turbulence in heated channel flow of supercritical fluids', Physics of Fluids, p.015108, 30(1).

\subsection{Introduction}

It is well established that heating fluids at a state above the thermodynamic critical pressure in environments where turbulent forced convection is expected to govern the flow development yields heat transfer rates that may deviate significantly from rates 
predicted with empirical correlations calibrated for subcritical fluids (Pioro \& Duffy, 2005; Duffy \& Pioro, 2005). Deteriorated heat transfer (DHT) is said to occur when the heat transfer rate is significantly lower than the predicted value for wall-temperaturecontrolled conditions, or the wall temperature is significantly higher than the predicted value for wall-heat-flux-controlled conditions. Conversely, improved or enhanced heat transfer (IHT) occurs when the heat transfer rate is higher than predicted, or the wall temperature is lower than predicted. These deviations in heat transfer rates often occur near the pseudo-critical state of the fluid, where the thermophysical properties have significant temperature dependence as illustrated in Figure 5-1. The pseudo-critical state is defined by the temperature at which the isobaric specific heat, $C_{p}$, is at its maximum value for a given pressure. The critical and pseudo-critical states are hereafter identified by the subscripts $c$ and $p c$, respectively.

Over the past few decades, heat-transfer research with supercritical fluids has focused on predicting the conditions that are conducive to phenomena that lead to heat transfer

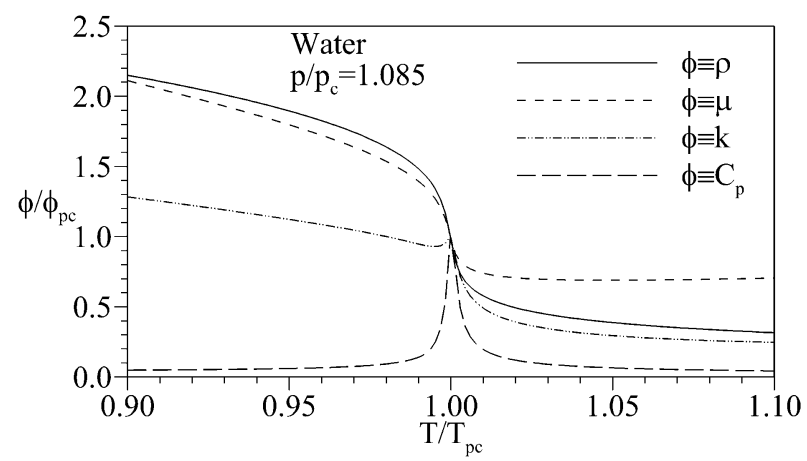

Figure 5-1: Temperature dependence of density, $\rho$, dynamic viscosity, $\mu$, thermal conductivity, $k$, and isobaric specific heat, $C_{p}$, of water in the vicinity of the pseudocritical state. 
variations (McEligot \& Jackson, 2004; Jackson, 2013; Pioro ${ }^{\mathrm{b}}$ et al., 2004) and the magnitude of such variations (Mokry et al., 2011; Shitsman, 1973). However, the onset of DHT and IHT is not predicted accurately over ranges of flow and thermal conditions with different fluids, and the heat-transfer models do not accurately predict the temperature of the heated wall under DHT. Experiments geared towards characterizing the underlying phenomena that drive DHT and IHT have been limited due to the complexity of such setups for working fluids at the supercritical state. The physical phenomena that drive the self-sustaining turbulent state of single-phase subcritical wall-bounded flows have become more evident in recent times through advancements in flow measurement techniques, and in particular through the availability of DNS data (Panton, 2001; Smits \& Delo, 2001; Adrian et al. 2000). These datasets reveal that self-replicating vortices resembling the shape of a hairpin, illustrated in Figure 5-2, are the dominant coherent structures in turbulent flows, and much of the characteristics of such flows can be explained in terms of the creation, growth, mutual interaction, and eventual degradation of these flow structures (Wu \& Moin, 2009; Adrian et al. 2000).

\subsubsection{Turbulence in Single-Phase Subcritical Wall-Bounded Flows}

A primary hairpin vortex may form in close proximity to the wall through streamwise grouping and subsequent roll-up of spanwise vorticity driven by inviscid and/or viscous flow instabilities (Kachanov, 1994). As indicated in Figure 5-2, the topology, orientation, and sense of rotation of vorticity of the hairpin vortex is such that fluid is transported away from the wall (ejection) between the legs of the vortex, and transferred towards the 


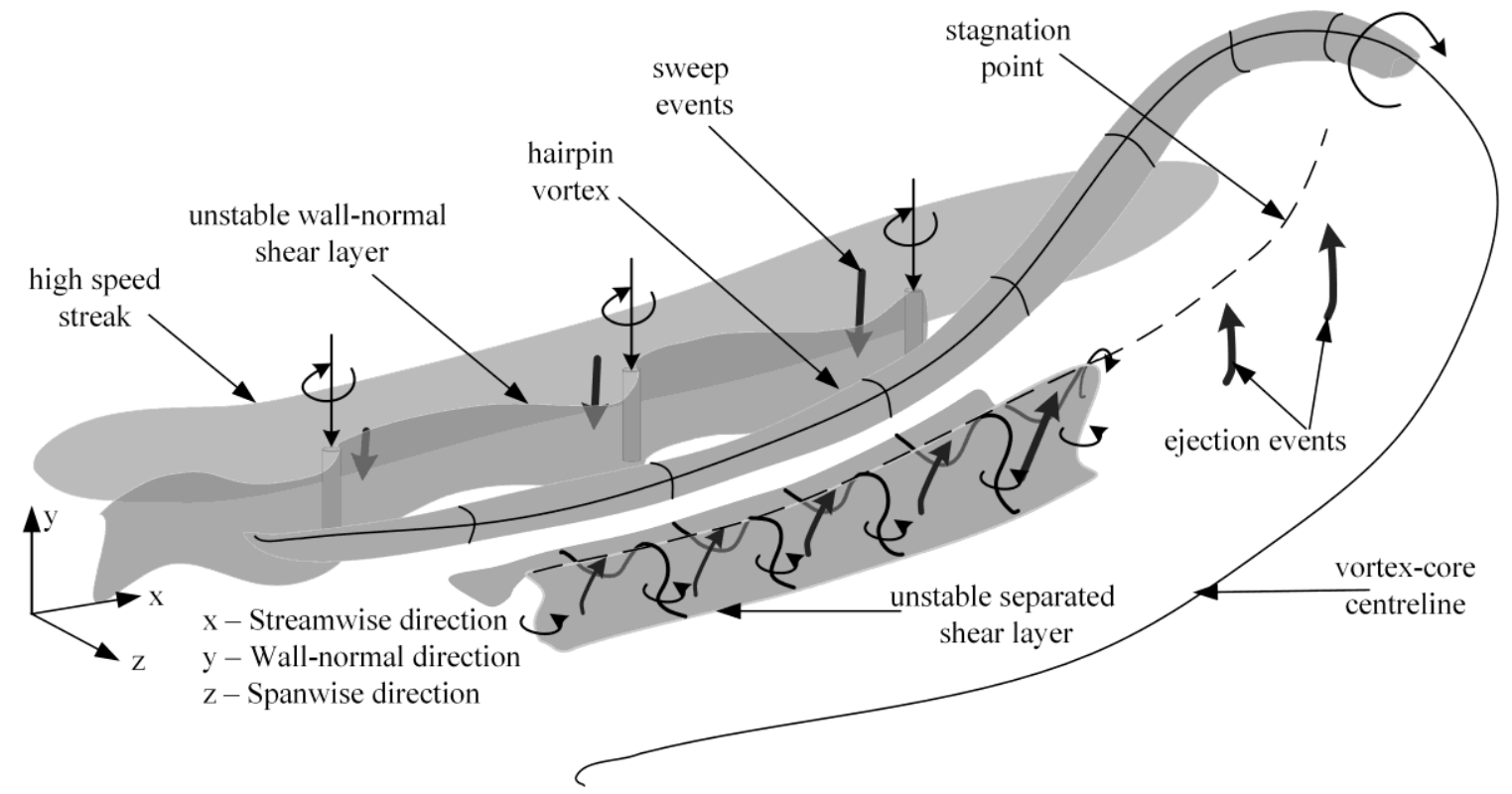

Figure 5-2: Schematic of a hairpin vortex in wall-bounded shear layers and its induced motions (adapted from Brinkerhoff (2014)).

wall (sweep) on the outboard sides of the legs. Due to the mean shear imposed on the flow by the presence of the wall, these ejection and sweep motions give rise to localized streamwise-aligned regions of streamwise momentum excess and deficit that are termed high- and low-speed streaks, respectively. Multiple mechanisms have been observed by which a mature hairpin vortex may create secondary hairpin vortices The mechanisms either involve inviscid instability of the separated shear layer that forms behind the primary/parent vortex (e.g. Singer and Joslin, 1994; Zhou et al. 1999) or instability of accumulated wall-normal vorticity on the outboard side of the hairpin vortices (e.g. Bernard et al. 1993; Schroder et al., 2004, 2008). Intricate details on the development of these instabilities to form hairpin vortices are discussed in Section 1.3.3. Through the same mechanism, the secondary hairpin vortex may create a tertiary hairpin vortex, and 
so on. This sequence of hairpin-vortex generation produces a group of approximatelystreamwise-aligned vortices denoted as a wavepacket (Adrian et al., 2000).

Within a wavepacket, the older, and hence taller, vortices extend to regions of higher velocity in the boundary layer, and therefore tend to convect downstream at higher speeds than the younger vortices. As such, streamwise interaction of neighbouring hairpin vortices within a wavepacket is rare (Adrian et al., 2000; Tomkins \& Adrian, 2003). On the other hand, complex interactions are observed between wavepackets and their respective hairpin vortices, which are promoted by the spanwise growth and spanwise meandering of the wavepackets, and by the streamwise interaction of wavepackets due to their varying convective speeds. For example, the adjacent legs of spanwise-interacting hairpin vortices may break down into smaller vortical structures owing to their opposite directions of rotation while the heads may merge to form a hairpin vortex of greater spanwise extent (Adrian et al., 2000; Tomkins \& Adrian, 2003). Tomkins \& Adrian (2003) observe that as the heads of these vortices merge, the low speed streaks associated with the wavepackets of the merging vortices also merge and form a single low-speed streak with a larger spanwise width. These authors noted that most of the near-wall lowspeed streaks, observed through their Particle Image Velocimetry (PIV) measurements in a fully-turbulent boundary layer, had begun to undergo this merging process by $y^{+}=100$. The resultant flow topology is one of multiple generations of wavepackets whereby smaller wavepackets near the wall exist within larger wavepackets residing farther away from the wall, which in turn exist within even larger wavepackets (Adrian et al., 2000). The number of generations of wavepackets is determined by the friction Reynolds 
number of the flow, $\operatorname{Re}_{\tau} \approx \delta / \delta_{v}$, which compares the scale of the larger coherent structures, $\delta$, to that of the smaller near-wall, $\delta_{v}=v_{w} / u_{\tau}$. As the number of incidences of vortex interaction increases, the population of fragmented vortices in the boundary layer also increases, thus making visualization of coherent motions more difficult with increasing Reynolds number. (Adrian et al., 2007; Jimenez et al., 2010). However, in close vicinity of the wall where the population of fragmented vortices is relatively smaller, a readily and consistently observed feature in low-, moderate-, and high-Reynolds-number flows is the spanwise-alternating regions of streamwise-elongated low- and high-speed streaks induced by first generation of wavepackets (Marusic et al., 2010). The mean spanwise spacing of the hairpin vortices of this first generation wavepackets and their associated low-speed streaks is $\Delta z^{+} \approx 100 \pm 20$. This spanwise spacing corresponds to the wavelength of perturbations that yields the highest growth rate in the kinetic energy of the perturbations over a period not greater than the local eddy-turnover time, and represents a balance of viscous dissipation and the kinetic energy of the transient wall-normal motions (Baig \& Chernyshenko, 2004; Buttler \& Farrell, 1993).

\subsubsection{Turbulence in Heated Flows of Supercritical Fluids}

Alterations to the above-described turbulence dynamics in a wall-bounded heated forcedconvection environment where the working fluid is at the supercritical state is the subject of the present study. DNS studies on heat transfer in flows of supercritical fluids has been performed by Azih et al. (2012), Reinink and Yaras (2015), and Bae et al. (2005, 2008), Nemati et al. (2015, 2016), Peeters et al. (2016), and Zonta et al. (2012a, 2012b). Insight into the mechanisms through which such flows are affected by spatial gradients of the thermophysical properties may be attained by investigating the development of the 
coherent structures of turbulence, as well as investigating statistical turbulence quantities (Corino and Brodkey, 1969). Through analysis of mean velocities, temperatures, Reynolds stresses, and turbulence intensities, Zonta et al. (2012a) noted that the density effects dominated those of other thermophysical properties for $\mathrm{Re}_{\tau}>180$. This observation is consistent with other studies of heated flows of supercritical fluids (Reinink and Yaras, 2015; Peeters et al., 2016).

IHT is typically observed in flows with comparatively low values of heat flux where density gradients are expected to be milder. Azih et al. (2012). Reinink and Yaras (2015) observed the development of turbulence along a heated wall at fluid temperatures just above the pseudo-critical temperature under flow conditions that yielded IHT. They observed notable wall-normal and spanwise density gradients existing in the vicinity of the inviscid unstable shear layers along the perimeter of near-wall low-speed streaks. These density gradients promote vorticity generation via the baroclinic instability mechanism which accelerates the inviscid instability mechanisms that lead to roll-up of the shear layer to form secondary vortices. A consequence of this accelerated roll-up is that coherent vortical structures are created at a faster rate and higher frequency, hence reducing the time-scale of development of small-scale turbulent motions that dictate the spanwise wavelength of the perturbations attaining optimum growth rate. Thus, wavepackets of hairpin vortices were observed with reduced streamwise and spanwise spacing, which enhanced heat transfer through increased wall-normal thermal mixing. 
When the flow is heated such that spatial gradients of density become much larger, the intensity of turbulence may deteriorate in close vicinity of the wall (Zonta et al., 2012b). Analysis of the root-mean-square (rms) of the streamwise component of the perturbation velocity and the wall-normal profiles of turbulence kinetic energy reveal that in the absence of buoyancy forces, turbulent motions are initially attenuated at the onset of heating and then slowly recover farther downstream (Nemati et al., 2015; Bae et al., 2008) The rates of attenuation/recovery of turbulence may be enhanced or slowed down by the presence of buoyancy forces. Upon heating in vicinity of the pseudo-critical state, near-wall flow acceleration due to significant volumetric expansion occurs in the wallnormal region where viscous dissipation dominates thus increasing the strain rate. Conversely, slightly farther away from the wall where production of turbulence kinetic energy dominates, the mean strain rate decreases thus resulting in relatively lower production rates compared to the dissipation rate of turbulence kinetic energy (Nemati et al. 2015, 2016; Zonta et al., 2012b). The attenuation of turbulence is supplemented in upward flow where the orientation of gravitational acceleration is such that the streamwise convection of lower-density low-speed streaks is less opposed by the buoyancy force than that of the surrounding higher-density high-speed fluid (Bae et al., 2005; Bae et al., 2008). Furthermore, local flow acceleration and wall-normal/spanwise density gradients in the vicinity of these streaks are demonstrated to counteract the generation of streamwise vorticity, which comprise the primary component of vorticity in the legs of hairpin vortices associated with these streaks. (Peeters et al., 2016). These phenomena reduce the intensity of the shear layer between the low- and high-speed streaks in the streamwise/spanwise plane, the instability of which is integral to the 
regeneration cycle of turbulence as previously discussed. As turbulence recovers from the deteriorated state, ejection of high-speed fluid is noted to be prevalent (Bae et al., 2008), which is contrary to the ejection of low-speed fluid that dominates constant property flows. Also, Instances of smaller-scale structures that form in the shear layer between lower-temperature high-speed and higher-temperature low-speed streaks have been observed. (Nemati et al., 2016). These structures may play a role in the recovery of turbulence as they may be associated with accelerated roll-up of the shear layer via the baroclinic instability mechanism as per the observations of Reinink and Yaras (2015). In heated downward flows buoyancy forces are observed to destabilize the flow, which is evident as increased contributions of the buoyancy terms to the overall rate of turbulent kinetic energy production (Nemati et al., 2016) Thus, buoyancy forces act to enhance heat transfer in such flows.

The forgoing description of fluid flow and heat transfer at the supercritical state has not distinguished between studies with isothermal walls (Nemati et al., 2015; Zonta et al., 2012a'; Zonta et al., 2012b) and those with iso-heat-flux walls (Reinink and Yaras., 2015; Azih et al., 2012; Bae et al., 2008; Bae et al., 2005). Nemati et al. (2016) show that for heat transfer in vicinity of the pseudo-critical state, a significant increase in wallnormal heat transfer due to turbulence advection occurs for iso-heat-flux walls in comparison to iso-thermal walls.

DNS studies of fully turbulent flows of supercritical fluid to date have focused on interpretation of averaged flow quantities which have not been sufficient to definitively 
shed light into the flow's physical structure. The present study identifies physical mechanisms through which the unique phenomena in heated flows of supercritical fluid occur and consequently identifies fluid and flow conditions that are conducive to these phenomena.

\subsection{Computational Method}

\subsubsection{Test Cases}

Five direct numerical simulations that capture the development of the water flow field within a channel of rectilinear geometry are performed at an inflow Reynolds number of 12,000 based on the channel hydraulic diameter, mass flow rate, and bulk-fluid viscosity. The friction Reynolds number at the inflow boundary has a value of $R e_{\tau}=\delta / \delta_{v}=225$, where $\delta$ is the channel half-height. The parameters that uniquely define each simulation are listed in Table 5-1. In the first simulation, named $Q 0 \_g 0 \_\phi f x d$, the flow is developed without heated walls and in the absence of buoyancy forces to a fully-developed statistically-steady turbulent state at a thermodynamic state defined by pressure and

Table 5-1: Simulation parameters for turbulent channel flow cases.

\begin{tabular}{|c|c|c|c|c|c|}
\hline Name of case & $\begin{array}{l}Q_{w}^{\prime \prime} / G \\
(\mathrm{~kJ} / \mathrm{kg})\end{array}$ & $\begin{array}{l}\text { Buoyancy } \\
\text { parameter }\end{array}$ & $\begin{array}{l}\text { Acceleration } \\
\text { parameter }\end{array}$ & $\begin{array}{l}\text { Varied fluid } \\
\text { properties }\end{array}$ & $\begin{array}{l}\text { Direction of } \\
\text { gravitational }\end{array}$ \\
\hline Q0_g0_tfxd & 0 & 0 & 0 & None & N/A \\
\hline Q3_g0_pfxd & 3.0 & 0 & 0 & None & N/A \\
\hline Q3_g0_pvar & 3.0 & 0 & 9.8 & All & N/A \\
\hline Q3_gy_ $\phi v a r$ & 3.0 & 32 & 9.8 & All & Wall-normal $(-y)$ \\
\hline Q3 gx $\phi v a r$ & 3.0 & 32 & 9.8 & All & Downstream $(+x)$ \\
\hline
\end{tabular}


temperature values of $p / p_{c}=1.085$ and $T / T_{p c}=0.983$, respectively. For water, the critical values of pressure and temperature are, $p_{c}=22.06 \mathrm{MPa}$ and $T_{p c}=654.4 \mathrm{~K}$. The results of this simulation provide the initial flow field from which the subsequent four simulations are developed. In these four simulations the channel walls are uniformly heated such that the wall heat-flux $\left(Q_{w}^{\prime \prime}\right)$ to channel mass flux $(G)$ ratio has a value of $3 \mathrm{~kJ} / \mathrm{kg}$, yielding wall-temperature values ranging from $T_{w}<T_{p c}$ to $T_{w}>T_{p c}$ along the length of the channel. In the simulation named $Q 3 \_g 0 \_\phi f x d$, the fluid thermophysical properties are fixed at values corresponding to the thermodynamic state of the $Q 0 \_g 0 \_\phi f x d$ simulation, and are therefore independent of the local changes in temperature and pressure. For the remaining three simulations, namely $Q 3 \_g 0 \_\phi v a r, Q 3 \_g y \_\phi v a r$ and $Q 3 \_g x \_\phi v a r$, the fluid properties are allowed to vary in accordance with the International Association for the Properties of Water and Steam (IAPWS) equations of state (IAPWS, 2009) Buoyancy forces are absent in the simulation named $Q 3 \_g 0 \_\phi v a r$. For the simulation named Q3_gy_ $\phi v a r$, the gravitational acceleration is oriented in the wall-normal direction, while for the simulation named $Q 3 \_g x \_\phi v a r$ it is oriented in the downstream direction. Also tabulated in Table 5-1 are an acceleration parameter $(A c)$, and a buoyancy parameter (Bo), serving as reference indicators for the onset of significant inertia-induced and buoyancy-induced turbulence phenomena, respectively (McEligot \& Jackson, 2004). These parameters are expressed as:

$$
\begin{gathered}
A c=4 \frac{\beta_{b} \dot{Q}_{w}^{\prime \prime}}{G C_{p, b}} \frac{1}{R e_{d} P r_{b}^{0.4}} \\
B o=\frac{R a}{R e_{d}^{3.425} P r_{b}^{1.8}}
\end{gathered}
$$


and the Rayleigh number is defined as:

$$
R a=\frac{g \beta_{b}\left(T_{w}-T_{b}\right) d_{h}^{3} \operatorname{Pr}}{v_{b}^{2}}
$$

where $g$ is the gravitational acceleration; $\beta$ is the volumetric expansion coefficient; $\mu$ is the dynamic viscosity; $G$ is the channel mass flux; $P r$ is the Prandtl number; $d_{h}$ is the hydraulic diameter; and, $R e_{d}$ is the Reynolds number based on the hydraulic diameter, bulk-fluid dynamic viscosity, and channel mass flux. The subscripts $b$ and $w$ indicate that the properties are evaluated at the bulk-fluid and heated-wall conditions, respectively. The heated-wall temperature is estimated from the expression calibrated for heat transfer in flows of subcritical single-phase fluids with negligible buoyancy effects:

$$
T_{w}=43 \frac{\dot{Q}_{w}^{\prime \prime} d_{h}}{k_{b} \operatorname{Re}_{d}^{0.8} \operatorname{Pr}_{b}^{0.4}}+T_{b}
$$

where the bulk fluid temperature, $T_{b}$, is obtained based on the pressure and streamwiselocal bulk fluid enthalpy (McEligot \& Jackson, 2004). Based on heat transfer data with subcritical fluids, forced convection heat transfer is expected for $R a<2 \times 10^{9}$ at the inflow Reynolds number of the present study; otherwise, mixed convection heat transfer is expected (Metaias \& Eckert, 1964). A gravitational acceleration value of $9.81 \times 10^{-3} \mathrm{~m} / \mathrm{s}^{2}$ is specified to yield $R a=7 \times 10^{8}$ such that forced convection heat transfer is the predicted heat-transfer mode for the present studies. For heat transfer with supercritical fluids, nonnegligible buoyancy- and inertia-induced phenomena are expected for $B o>6 \times 10^{7}$ and $A c>9.5 \times 10^{7}$, respectively (McEligot \& Jackson, 2004). The values of $B o$ and $A c$ tabulated in Table 5-1 are evaluated at the pseudo-critical state and suggest that nonnegligible inertial effects are expected in the cases where the fluid properties are varied, 
and non-negligible buoyancy effects are expected in the cases with gravitational acceleration.

\subsubsection{Computational Domains}

Schematics of the computational domains used for the cases listed in Table 5-1 are presented in Figure 5-3. Figure 5-3 (a) shows the computational domain for the Q0_g0_ $\phi f x d$ case and Figure 5-3 (b) shows the computational domain for the remaining cases. To facilitate comparison of the setup parameters for the computational domains and the results of these cases, two methods are employed for data normalization. The first method of normalization involving wall variables is indicated with a "+" superscript; unless otherwise specified, streamwise-local wall variables are used. The second method of normalization is indicated by the " $\delta$ " subscript, and reflects normalization based on larger-scale variables. These larger-scale variables are the channel half-height, $\delta$, the time-mean of the streamwise-local area-averaged $\mathrm{x}$-component of velocity, $\langle u\rangle$, and the

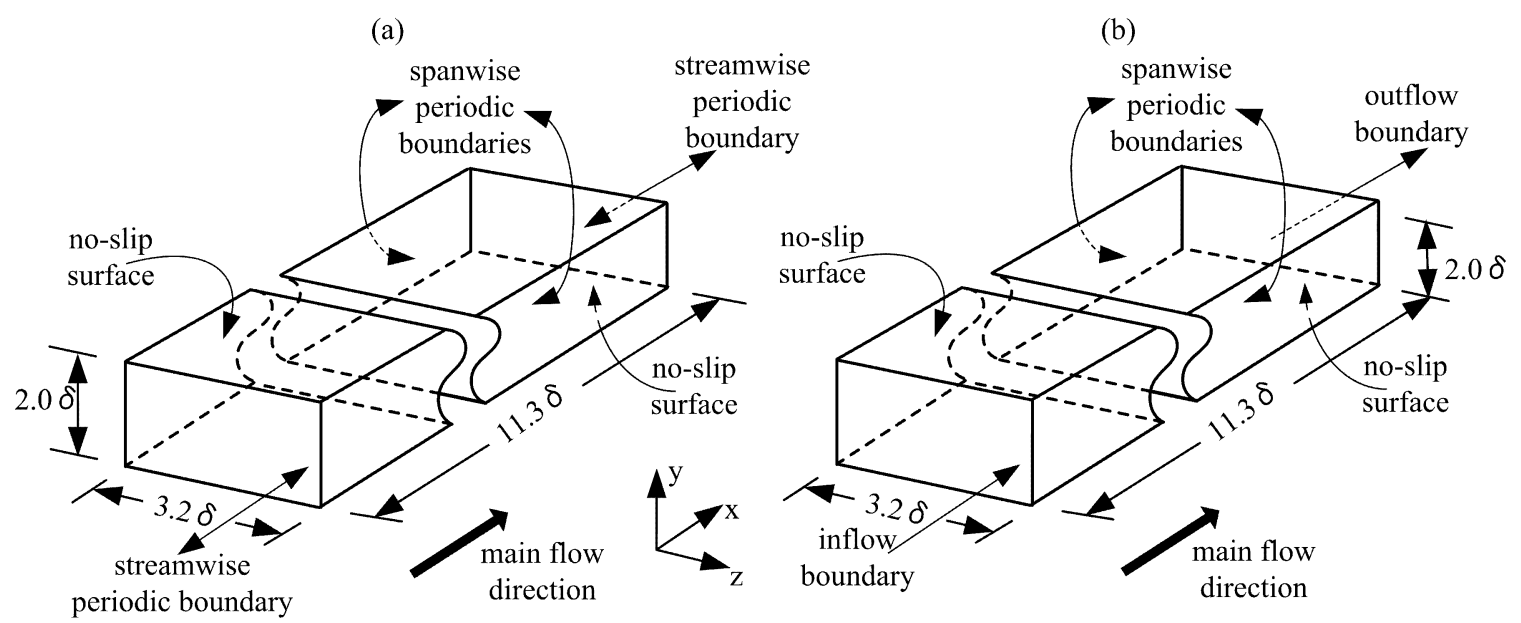

Figure 5-3: Computational domains: a) domain for generating initial conditions for heated-wall simulations; b) domain for channel flow with heated walls. 
time-mean streamwise-local centreline temperature. The streamwise-local area and time average of a quantity $\phi$ is denoted by angled brackets, $\langle\phi\rangle$, and is obtained over the selected spanwise/wall-normal plane that is bounded by the $y=0$ and $y=\delta$.

The half-height of the channel is set to a value of $\delta=22.7 \mathrm{~mm}$. Chin et al. (2010) show that, at the friction Reynolds number of the present study, a domain length greater than $25 \delta$ is required to capture all scales of coherent flow structures of turbulence within a channel. The validity of using shorter domains and hence not resolving the largest scales is discussed in detail by Jimenez et al. $(1999,1991)$. For a given streamwise length of the computational domain, the wall-normal height within which the coherent flow structures of turbulence are accurately resolved corresponds with the largest wall-normal distance at which two-point correlations of the streamwise perturbation velocity component decrease to a zero value within half the streamwise length of the computational domain (Chin et al. 2010, Jimenez \& Moin, 1991). Here the perturbation velocity field is obtained by subtracting the time-mean velocity field from the instantaneous velocity field. Analysis of two-point correlations of the streamwise perturbation velocity component $\left(R_{u^{\prime} u^{\prime}}\right)$ reveals that the streamwise extent of the computational domain for the simulations is sufficient to accurately capture the development of the coherent flow structures developing below a wall-normal distance of $y_{\delta}=0.60$ as indicated for the Q3_g0_pvar case in Figure 5-4. The streamwise extent of the computational domain is deemed sufficient for the present study as several authors have observed that heated turbulent flows with supercritical working fluids are dominantly affected by near-wall flow phenomena (Azih et al., 2012; Bae et al., 2008; Li et al., 2007; Bae et al., 2005). To ensure that the coherent flow structures are self 
sustaining, the span of the computational domain must adhere to $L_{z}^{+}>220$ (Jimenez \& Moin, 1991). The domain span of $\Delta z^{+}>740(\Delta z=3.2 \delta)$ for the present study is therefore quite conservative.

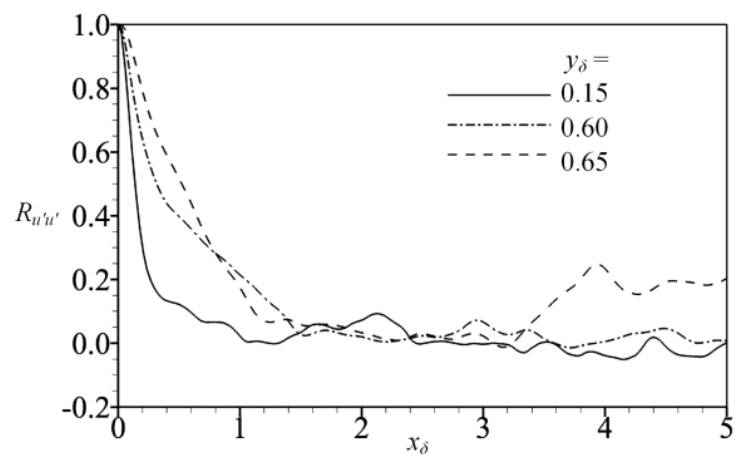

Figure 5-4: Two-point correlations of the streamwise component of fluctuation velocity for the non-buoyant variable-property case.

\subsubsection{Boundary and Initial Conditions}

The boundary and initial conditions for the $Q 0 \_g 0 \_\phi f x d$ simulation are described first as this case provides inflow and initialization data for the remaining cases. The $Q 0 \_g 0 \_\phi f x d$ simulation is developed to a statistically-steady state by the use of streamwise periodic boundaries starting from a turbulent initial condition. This initial turbulent flow field, which serves to accelerate convergence to a statistically steady solution, is obtained from the simulation with air as the working fluid described in Chapter 4, where a turbulent boundary layer over an unheated flat surface was simulated using streamwise periodic boundaries. Streamwise periodic boundaries cause the temporal development of the boundary layer over the flat wall to occur in a spatially uniform manner. The flow field at $R e_{\tau}=225$ from this flat-wall simulation is mapped into the $Q 0 \_g 0 \_\phi f x d$ simulation in $\delta$ normalized Cartesian space. The mapped velocity field is periodically repeated in the 
streamwise direction until the outlet boundary of the $Q 0 \_g 0 \_\phi f x d$ is reached. All components of the velocity field from the flat-wall simulation is rescaled using a globally uniform scaling factor to ensure consistency with the area-averaged mass flux of $7.94 \mathrm{~kg} /\left(\mathrm{s} \mathrm{m}^{2}\right)$ of the $Q 0 \_g 0 \_\phi f x d$ simulation. At the streamwise-periodic boundaries of the $Q 0 \_g 0 \_\phi f x d$ simulation only, the velocities are matched between corresponding nodes of the two boundaries. The mass flux is allowed to vary over the streamwise-periodic interface but is constrained to have an area-averaged value of $7.94 \mathrm{~kg} /\left(\mathrm{s} \mathrm{m}^{2}\right)$. The cases with heated walls are initialized with a flow field corresponding to the final timestep of the $Q 0 \_g 0 \_\phi f x d$ simulation. Inflow velocity data for these simulations are sequentially extracted in a temporally continuous manner from the $Q 0 \_g 0 \_\phi f x d$ and the $Q 3 \_g 0 \_\phi f x d$ simulation. The initial set of inflow data is obtained for a duration of one through-flow time from the $Q 0 \_g 0 \_\phi f x d$ simulation at the plane defined by $x_{\delta}=9$. The subsequent set of inflow velocity data is extracted from the plane corresponding to $x_{\delta}=9$ in the Q3_g0_hfxd simulation, for which the temperature and velocity fields are decoupled, thus ensuring the temporal continuity of the inflow velocity field supplied to the heated cases. For all cases, spanwise periodic boundaries are applied in a manner such that all of the primitive variables are mapped between corresponding nodes of this periodic interface. A no-slip boundary condition is applied on the $y=0$ and $y=2 \delta$ walls of the channel, respectively denoted as the lower wall and upper wall henceforth. At the inflow plane of the cases with heated walls, a time-independent and spatially-uniform temperature of $T / T_{p c}=0.983$ is specified. For the cases where the thermophysical properties of the fluid are varied, this approximation for the inflow temperature introduces artificial conditions in the near-wall sections of the computational domain in close vicinity of the inflow 
plane. A simulation was run at the conditions of the $Q 3 \_g 0 \_\phi v a r$ case but with the heated wall placed $0.5 \delta$ downstream of the inflow boundary. This simulation showed that the effect of the artificial conditions of the present setup is to induce larger temperature gradients ( $\sim 6 \%$ increase) and more rapid acceleration of the near-wall fluid $(\sim 17 \%$ increase) at the onset of heating of the fluid. However, the development of the coherent structures were observed to be almost identical in both cases, the difference being the slightly faster evolution of the fluid dynamics in the case with the heated wall commencing at the inflow plane. Likewise, Bae et al. (2005) showed the downstream effects of this inflow approximation on their computed flow field to be insignificant by comparing their computed results to experiments that used heated gas flows that featured large spatial variations of the gas properties. At the outflow boundary, a static pressure value of $p / p_{c}=1.085$ is kept temporally constant in an area-averaged sense. To eliminate artificial effects of this outflow boundary condition on the flow turbulence information gleaned from the simulation, this boundary is placed $2.3 \delta$ downstream from the region of interest. The adequacy of the $2.3 \delta$ streamwise distance between the outflow boundary and the region of interest is addressed in Section 5.3. A spatially uniform wall heat flux value of $25 \mathrm{~kW} / \mathrm{m}^{2}$ is specified at the no-slip walls of the cases with heated walls, thus yielding a wall heat-flux to channel mass-flux ratio of $3 \mathrm{~kJ} / \mathrm{kg}$.

\subsubsection{Spatial and Temporal Grids}

Structured grids with hexahedral cells are mapped onto the computational domains shown in Figure 5-3. The node distributions must be selected such that they capture the smallest eddies in the flow which scale on the Kolmogorov length scale, $\eta$ (Friedrich et al., 2001). Indeed, the turbulence kinetic energy dissipation occurs through smaller-scale 
eddies with length scales of $6 \eta$ to $15 \eta$ (Tanahashi et al., 2004; Moin \& Mahesh, 1998). The smallest thermal scales, represented by the Batchelor length scale $\left(\eta_{\theta}=\eta \operatorname{Pr}^{-0.5}\right)$, are expected to be smaller than $\eta$ since the magnitude of the Prandtl number exceeds unity in the present simulations $(1.67<\operatorname{Pr}<11.2)$. The grid spacings normalized by the viscous length scale and Batchelor length scale are provided in Table 5-2 and Table 5-3.

Table 5-2: Node spacings corresponding to the maximum value in vicinity of the wall.

\begin{tabular}{|c|c|c|c|c|c|c|}
\hline Name of case & $\overline{\Delta \Delta \mathrm{x}^{+}}$ & $\overline{\Delta \Delta \mathrm{y}^{+}}$ & $\overline{\Delta \Delta \mathrm{z}^{+}}$ & $\overline{\Delta \Delta \mathrm{x} / \eta_{\theta}}$ & $\overline{\Delta \Delta \mathrm{y} / \eta_{\theta}}$ & $\overline{\Delta \mathrm{z} / \eta_{\theta}}$ \\
\hline$\overline{Q 0 \_g 0 \_\phi f x d}$ & 5.0 & 0.25 & 4.0 & - & - & - \\
\hline$Q 3 \_g 0 \_\phi f x d$ & 5.0 & 0.12 & 4.0 & 8.4 & 0.22 & 6.7 \\
\hline Q3_g0_ $\phi v a r$ & 9.5 & 0.22 & 7.6 & 15.9 & 0.37 & 12.8 \\
\hline Q3_gy_dvar (lower wall) & 10.1 & 0.24 & 8.0 & 16.8 & 0.40 & 13.4 \\
\hline Q3_gy_dvar (upper wall) & 5.5 & 0.13 & 4.4 & 9.2 & 0.42 & 7.4 \\
\hline$Q^{3} \_g x \_\phi v a r$ & 10.1 & 0.24 & 8.0 & 16.8 & 0.16 & 13.5 \\
\hline
\end{tabular}

Table 5-3: Range of wall-normal node spacings for computational domains.

\begin{tabular}{|c|c|c|}
\hline Name of case & $\Delta \mathrm{y}^{+}(\min -\max )$ & $\Delta \mathrm{y} / \eta_{\theta}{ }^{+}(\min -\max )$ \\
\hline$Q 0 \_g 0 \_\phi f x d$ & $0.25-3.06$ & - \\
\hline$Q 3 \_g 0 \_\phi f x d$ & $0.12-3.02$ & $0.22-6.10$ \\
\hline$Q 3 \_g 0 \_\phi v a r$ & $0.22-5.62$ & $0.37-8.13$ \\
\hline Q3_gy_dvar (lower wall) & $0.24-6.13$ & $0.40-8.33$ \\
\hline Q3_gy_dvar (upper wall) & $0.13-3.33$ & $0.42-7.53$ \\
\hline Q3_gx_pvar & $0.24-6.13$ & $0.16-8.80$ \\
\hline
\end{tabular}


For the $Q 0 \_g 0 \_\phi f x d$ case, the first node off the wall is located at $y^{+}=0.25$, and the wallnormal node spacing is increased at a constant rate between $0<y^{+}<20$, such that 17 nodes are placed below $y^{+}=10$. In the range of $20<y^{+}<150$, the node spacing follows $\Delta y^{+}=\left(0.41 y^{+}\right)^{1 / 4}$, which corresponds to the Kolmogorov length scale in the log-law region of wall-bounded turbulent shear layers. ${ }^{42}$ In the range of $y^{+}>150$ and $y \leq \delta$, the node spacing is increased at a rate of $10 \%$. For the cases with heated walls, the wall-normal node distribution is modified such that the first node off the wall is located at a $y^{+}$value of 0.12 , and the wall-normal node spacing is increased at a rate to place 23 nodes below $y^{+}=10$. The computational domains have 417 and 118 nodes in the streamwise spanwise directions. The computational domain with heated walls has 139 wall-normal nodes and the unheated computational domain has 133 wall-normal nodes. These spatial grids are comparable to those used in the studies of Bae et al. (2005) and Nemati et al. (2015).

A timestep size of $\Delta t^{+}<0.2$ is required to accurately capture the temporal development of coherent flow structures in wall-bounded turbulent shear layers; $t^{+}$is the time normalized by the viscous time scale, $v /\left(u_{\tau}\right)^{2}$ (Freidrich, 2001; Kong et al., 2000). A constant timestep value of $\Delta t^{+}=0.013$ is specified for the present cases. This choice of timestep is dictated by the numerical stability of the computational algorithm which is affected by the substantial spatial gradients in the thermophysical properties.

\subsubsection{Governing Equation and Solution Method}

The dimensional strong-conservation-law forms of the governing equations are given in tensor notation in Equation 5-5 to 5-7. 


$$
\begin{gathered}
\frac{\partial \rho}{\partial t}+\frac{\partial}{\partial x_{j}}\left(\rho u_{j}\right)=0 \\
\frac{\partial}{\partial t}\left(\rho u_{j}\right)+\frac{\partial}{\partial x_{j}}\left(\rho u_{j} u_{i}\right)=-\frac{\partial p}{\partial x_{i}}+\frac{\partial \tau_{i j}}{\partial x_{j}}+\rho g_{i} \\
\frac{\partial}{\partial t}\left(\rho h_{0}\right)+\frac{\partial}{\partial x_{j}}\left(\rho u_{j} h_{0}\right)=\frac{\partial p}{\partial t}+\frac{\partial}{\partial x_{j}}\left(k \frac{\partial T}{\partial x_{j}}\right)+\frac{\partial}{\partial x_{j}}\left(u_{i} \tau_{i j}\right)+\rho u_{i} g_{i}
\end{gathered}
$$

In these equations, $\rho$ is the working-fluid density; $u_{j}$ is the $j$ Cartesian component of velocity; $x_{j}$ is the $j$ component of Cartesian spatial coordinate; $p$ is the static pressure; $\tau_{i j}$ is the $i j$ component of the viscous stress tensor; $h_{0}$ is the total enthalpy; $k$ is the thermal conductivity; and, $T$ is the static temperature. The fluid is modelled as a Newtonian fluid such that the components of the viscous stress tensor relate to the strain rates through:

$$
\tau_{i j}=\mu\left(\frac{\partial u_{i}}{\partial x_{j}}+\frac{\partial u_{j}}{\partial x_{i}}-\frac{2}{3} \frac{\partial u_{k}}{\partial x_{k}} \delta_{i j}\right)
$$

where $\mu$ is the dynamic viscosity and $\delta_{i j}$ is Kronecker delta. Inherent in the formulation of Equations 5-6 to 5-8 is Stokes' hypothesis which effectively ignores the contribution of the bulk viscosity on the flow development. For wall-bounded turbulent flows, Cramer \& Bahmani (2014) demonstrate that this hypothesis is only valid if the ratio of the bulk viscosity to dynamic viscosity is less than $\left(R e_{\delta}\right)^{0.5}$. Given the $p / p_{c}$ value of 1.085 for the present study, the maximum value of the ratio of bulk viscosity to wall dynamic viscosity is approximately 50, and occurs at the pseudo-critical state. (Hasan \& Farouk, 2012). This value is about half of $\left(R e_{\delta}\right)^{0.5}$, therefore it is expected that the effects of bulk viscosity are negligible under the conditions of the present study.

The commercial software package ANSYS CFX ${ }^{\circledR}$ (Version 12) is used to perform the simulations. Using a finite volume technique, the solver discretizes the integral 
formulation of the governing equations with an equivalent of second-order centred differencing of the spatial derivatives and second-order backward-Euler differencing of the temporal derivatives (ANSYS, 2009). Relevant thermophysical properties are obtained via bilinear interpolation of the tabularized IAPWS equations of state for water (IAPWS, 2009), which are indexed by temperature and pressure. Solution convergence of the discretized equations at each timestep is obtained with outer iteration loops serving to converge the coefficients of the linearized governing equations and inner iteration loops facilitating the solution of these equations through W-type algebraic multigrid cycles (ANSYS, 2009). Six grid levels are used in the multigrid cycle. A single solution sweep is performed for each grid level during the restriction legs of the multigrid cycle, and three solution sweeps are performed for each grid level during the prolongation legs. The solution is obtained with one inner-loop iteration per outer-loop iteration, and multiple outer-loop iterations per timestep. For each timestep, the solution is declared as converged when the rms of the normalized residuals of the governing equations are reduced to less than $10^{-5}$, and this is realized with up to five outer-loop iterations per timestep. With the same computational algorithm as that of the present study, the studies of Brinkerhoff \& Yaras (2014), Dave et al. (2013), and Reinink \& Yaras (2015) indicate that a satisfactory level of convergence is achieved with rms residuals less than $10^{-5}$.

The simulations were partitioned and executed on 20 Intel $^{\mathrm{TM}}$ L5410 Xeon processors. Approximately 1300 viscous time units $(100,000$ timesteps) are required to reach a statistically-steady state for the $Q 0 \_g 0 \_\phi f x d$ simulation after which one through flow time is simulated to provide inflow data for the cases with heated walls. For the purposes 
of quantifying the duration of the simulations, the viscous time units are evaluated at the inflow boundary. Statistically-steady state was deemed achieved when the mean values of the streamwise velocity and the turbulence kinetic energy computed at $x_{\delta}=9$ using the last 25 viscous time units $(2,000$ timesteps) converged within $\pm 1 \%$. Using the velocity field at $t^{+}=1300$ of the $Q 0 \_g 0 \_\phi f x d$ case as an initial condition, approximately 200 viscous time units $(15,000$ timesteps $)$ are required to reach a statistically-steady state for the Q3 g0_ $\phi f x d$ case, after which 500 viscous time units (40,000 timesteps) are computed for flow analysis. For the $Q 3 \_g 0 \_\phi v a r, Q 3 \_g y \_\phi v a r$, and $Q 3 \_g x \_\phi v a r$ cases, 300 viscous time units $(25,000$ timesteps $)$ are required to reach a statistically-steady state from the same initial conditions, and then 400 viscous time units $(30,000$ timesteps) are computed for flow analysis. Statistically-steady data from 30,000 timesteps is sufficient to obtain reliable flow statistics and provide enough instances of flow structure development to characterize the flow dynamics of each of the computed cases.

\subsection{Validation of Computational Method}

In Figure 5-5, the distributions of mean velocity, rms perturbation velocity components, turbulence production and dissipation rates, and temperature of the $Q 3 \_g 0 \_\phi f x d$ case are compared to turbulent channel flow data available in the published literature. Here, the mean identified with an overbar refers to averages taken along statistically-homogeneous coordinates, which are the temporal and spanwise coordinates in the present study. Time- 

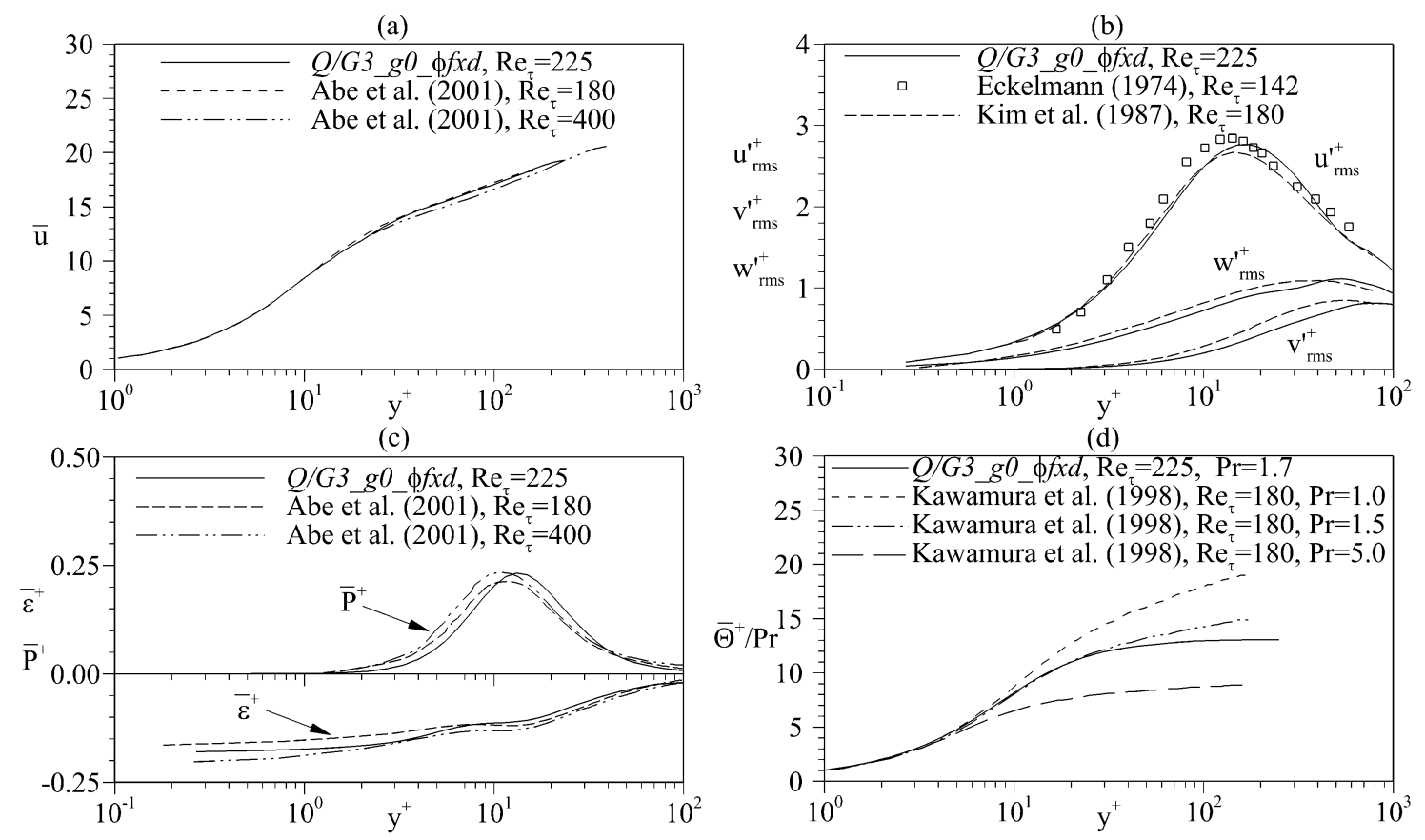

Figure 5-5: Mean flow properties evaluated at $x_{\delta}=9$ for the turbulent channel cases: a) streamwise velocity component; $b$ ) root-mean-square of perturbation velocity components; c) specific rates of turbulence kinetic energy production $\left(P^{+}\right)$and dissipation $\left(\varepsilon^{+}\right) ;$d) temperature.

averages are obtained with an interval corresponding to every $50^{\text {th }}$ timestep over the duration of the statistical-steady data and spanwise-averages are obtained using all available spanwise nodes for the corresponding timestep. For all of the heated-wall cases, the budgets of turbulence kinetic energy were observed to vary by less than $1 \%$ between values calculated using every $50^{\text {th }}$ timestep and those calculated with every timestep. The perturbation component of the velocity extracted from the velocity field using the Reynolds-averaging method is indicated by the (') superscript, and using the Favreaveraging method is indicated by the (") superscript. Abe et al. (2001) observed excellent agreement between two DNS datasets obtained using second-order and fourth-order finite difference schemes for spatial discretization, with a grid resolution of $\Delta x^{+} \approx 9$ and $\Delta z^{+} \approx 5$ 
for both discretization schemes. Abe et al.'s (2001) results shown in Figure 5-5 correspond to computations with the second-order scheme. Kim et al. (1987) solved the governing equations using a spectral method based on Fourier series in the streamwise and spanwise directions and Chebychev polynomial expansions in the wall-normal direction with a grid resolution of $\Delta x^{+} \approx 12$ and $\Delta z^{+} \approx 7$. Identical time advancement schemes were used by Abe et al. (2001) and Kim et al. (1987), which consisted of the second-order Crank-Nicolson method for implicit terms and the fourth- order AdamsBashforth method for explicit terms. The statistical turbulence properties of the

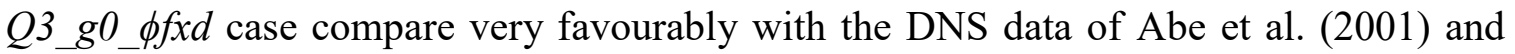
Kim et al. (1987), especially for $y^{+}<60$. Favourable agreement is also noted when results of the $Q 3 \_g 0 \_\phi f x d$ case are compared with the hot-film experimental data of Eckelmann (1974), as illustrated in Figure 5-5(b). Further validation of the computational method is realized by comparing the computed distribution of mean temperature at a plane defined by $x_{\delta}=9$ to the results of Kawamura et al.(1998), and is shown in Figure 5-5(d). Kawamura et al. (1998) studied the effect of the Prandtl number value on turbulent forced-convection heat transfer in a channel flow with $R e_{\tau}=180$ using a numerical scheme identical to that of Abe et al. (2001). In Figure 5-5(d), the normalized temperature, $\bar{\theta}^{+}=\left(\bar{T}_{w}-\bar{T}\right) / \bar{T}_{\tau}$ is used to define the ordinate, where $T_{\tau}=\dot{Q}_{w}^{\prime \prime} /\left(\rho_{w} C_{p, w} u_{\tau}\right)$ is the friction temperature. Figure 5-5(d) depicts favourable agreement in the inner part of the shear layer between the results of $Q 3 \_g 0 \_\phi f x d$ case for which $P r=1.67$ and Kawamura et al.'s (1998) case corresponding to a $\operatorname{Pr}=1.5$. The fuller temperature profile in the outer part of the shear layer for the $Q 3 \_g 0 \_f f x d$ case when compared to Kawamura et al.'s (1998) $P r=1.5$ case is expected due to the comparatively higher Reynolds number 
and Prandtl number of the $Q 3 \_g 0 \_\phi f x d$ case $^{52}$ (Kawamura et al., 1998; Kadar, 1981) as well as the fact that the heated cases of the present study are not thermally fully developed.

\subsection{Results and Discussion}

To provide a baseline for comparison to published studies (Peeters et al., 2016; Nemati et al., 2016; Nemati et al., 2015; Bae et al., 2008, 2005), the wall-normal profiles of the mean density and dynamic viscosity as well as those of the rms of the perturbation density and dynamic viscosity are presented in Figure 5-6. The wall-normal gradient of
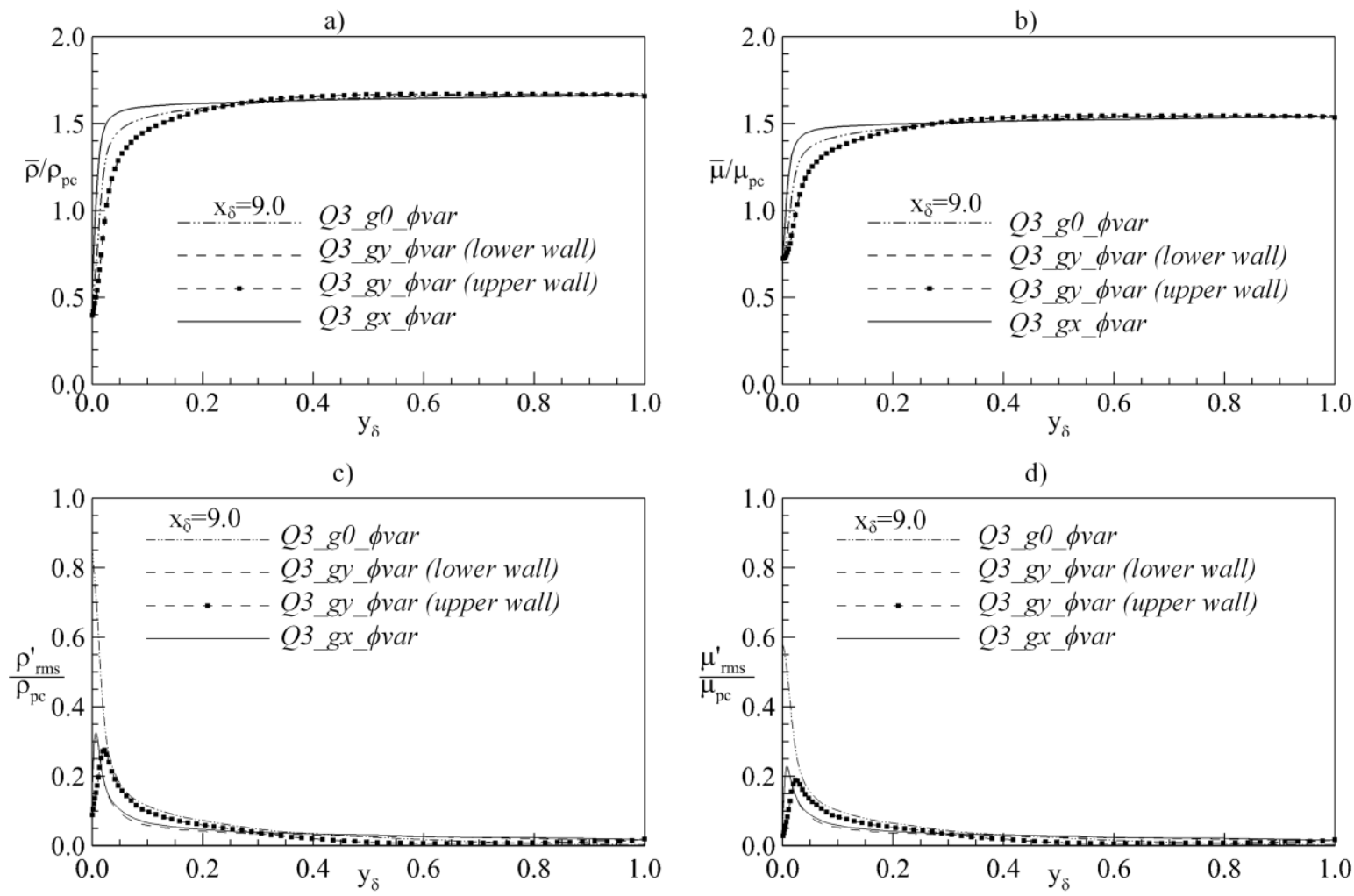

Figure 5-6: Wall-normal profile of density and viscosity at $x_{\delta}=9$ : a) mean density; b) mean viscosity; c) rms perturbation density; d) rms perturbation viscosity. 
the mean density and dynamic viscosity are higher in the near-wall region of the present study than those of the referenced published studies. Likewise, the peaks of the nondimensionalized rms perturbation density and dynamic viscosity of the present study are notably higher than those of Peeters et al. (2016) who report a peak value of approximately 0.16 and 0.15 , respectively, for their simulated cases. Flood-plots of thermophysical properties are shown in Figure 5-7 for the non-buoyant variable-property case. The contour illustrated with a solid line represents a constant-temperature contour at a value of $T / T_{p c}=0.99$. The strong similarity in the dynamic viscosity and density fields is consistent with the observations of Peeters et al. (2016) and is expected as per the similar temperature dependence of these two properties depicted in Figure 5-1. The spatial variation of thermal conductivity is small compared to those of dynamic viscosity, density, and the specific heat. Figure 5-7 indicates that the temperature contour plotted is a reasonable demarcation for the regions of high spatial variations of thermophysical properties.

As discussed in Section 5.1, the dominant coherent flow patterns in the near-wall region of turbulent channel flows are low- and high-speed streamwise-oriented velocity streaks, which are induced by wavepackets of hairpin vortices, having a mean spanwise spacing of $\Delta z^{+}=100 \pm 20$ (Smits \& Delo, 2001). The wall-normal perturbation velocity component, $v^{\prime \prime}$, is strongly influenced by the ejection and sweep motions that lead to the formation of streaks. Accordingly, in the present study, the mean spanwise wavelength of 

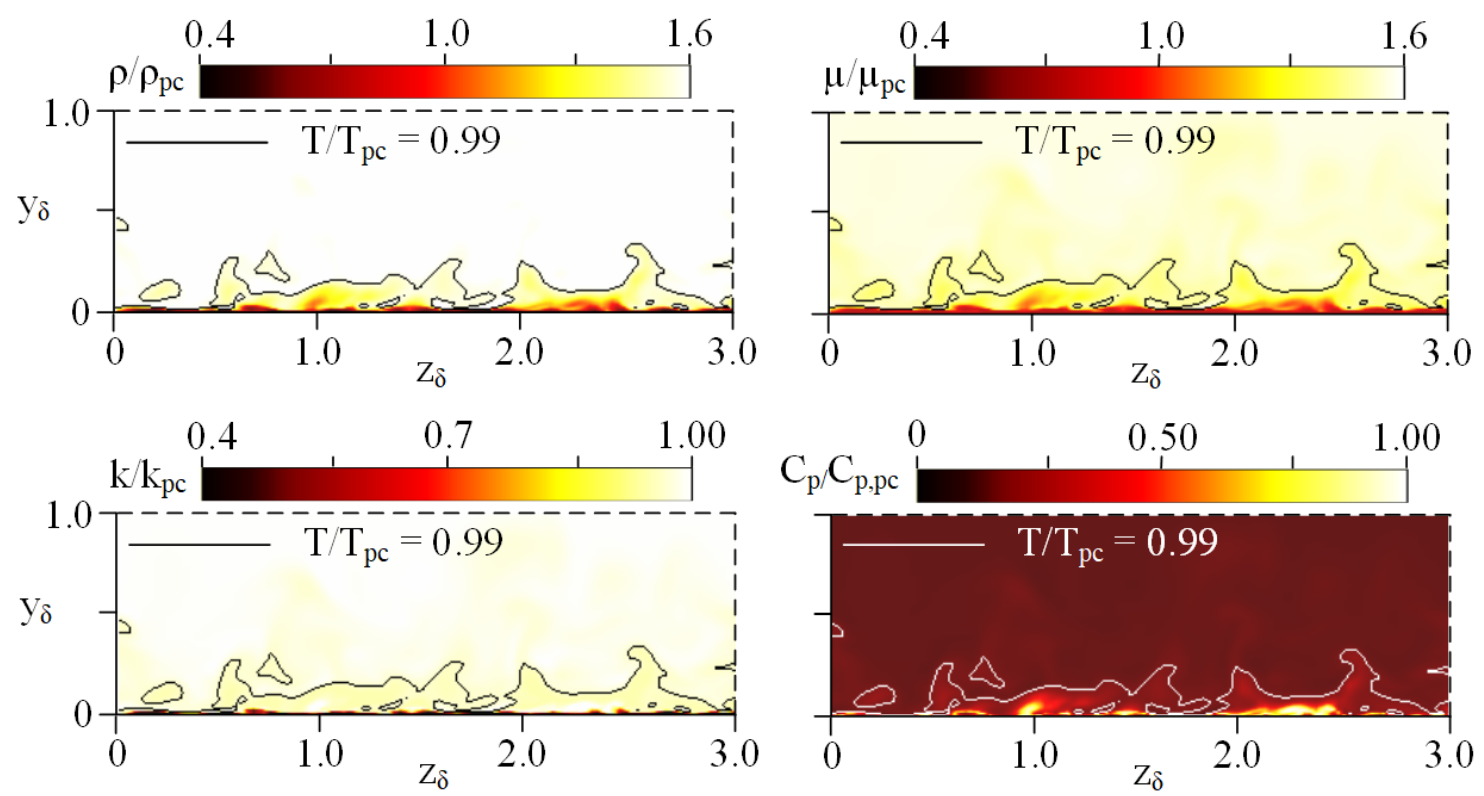

Figure 5-7: Flood-plots of the thermophysical properties in the non-buoyant variableproperty case at $\mathrm{x}_{\delta}=9$.

the streaks at a given streamwise location is obtained as the time-mean of the spanwise wavelength of the peak power spectral density (PSD) of $v^{\prime \prime}$ denoted by $\bar{\lambda}_{P S D_{-} v_{-}{ }_{1}}$. Unless otherwise specified, wavelength analyses are performed at $y^{+}=15$, which is in the range of wall-normal proximity where the production rate of turbulence kinetic energy is maximum. The values of $\bar{\lambda}_{P S D_{-} v_{-}{ }_{1}}$ at $x_{\delta}=9$ are presented in Table 5-4 together with a secondary wavelength, $\bar{\lambda}_{P S D_{-} v_{-} 2}$, which is the time-mean of the spanwise wavelength of the second highest peak in PSD values of $v^{\prime \prime}$. For the heated-wall, fixed-property case, the mean spanwise spacing of the streaks $\left(\Delta z^{+}=97 \pm 3, \Delta z_{\delta}=0.44 \pm 0.00\right)$ is within the range of values quoted in the published literature (Smits \& Delo, 2001). The quoted uncertainty in these spacing values represents the standard error of the mean. The results for the cases in which the thermophysical properties are allowed to vary suggest the presence of 
competing turbulence structures in the near-wall region. This is deduced from the similarity of the PSD amplitudes associated with the first and second most dominant spanwise wavelength for these cases when compared to the heated-wall, fixed-property case. Analysis of near-wall coherent flow structures is presented next to provide insight into the mechanisms that result in the trends indicated in Table 5-4.

\subsubsection{Coherent Flow Structures in the Absence of Spatial Variations in Thermophysical Properties}

This section discusses the development of coherent flow structures in the absence of spatial variations in thermophysical properties as per the results of the heated-wall, fixedproperty simulation. The discussions provided herein reinforce the well-established dynamics of the coherent structures of turbulence in such flows, reviewed in Section 5.1, and will serve as a baseline for the remaining cases where the thermophysical properties

Table 5-4: Spanwise wavelength of the dominant coherent flow structures at $y^{+}=15$, $x_{\delta}=9.1-$ most dominant wavelength; $2-2^{\text {nd }}$ most dominant wavelength.

\begin{tabular}{|c|c|c|c|}
\hline \multirow[t]{2}{*}{ Case } & $\bar{\lambda}_{P S D_{-} v^{\prime \prime}{ }_{1}}$ & $\overline{\bar{\lambda}}_{P S D_{-} v^{\prime \prime}{ }_{-}}$ & \multirow{2}{*}{$\frac{\overline{\overline{P S D}}\left(\lambda_{P S D_{-} v^{\prime \prime} 1}\right)}{\overline{P S D}\left(\lambda_{P S D_{-} v_{{ }_{-} 2}}\right)}$} \\
\hline & $\Delta z^{+}\left(z_{\delta}\right)$ & $\Delta z^{+}\left(z_{\delta}\right)$ & \\
\hline Q3_g0_dfxd & $97(0.44)$ & $195(0.88)$ & 42.1 \\
\hline 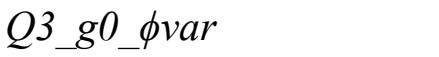 & $111(0.35)$ & $381(1.20)$ & 1.4 \\
\hline Q3_gy_фvar (lower wall) & $95(0.25)$ & $257(0.61)$ & 1.2 \\
\hline Q3_gy_фvar (upper wall) & $97(0.51)$ & $201(1.07)$ & 2.0 \\
\hline 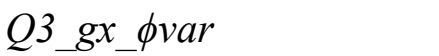 & $113(0.40)$ & $240(0.59)$ & 1.7 \\
\hline
\end{tabular}


are allowed to vary in the absence of a buoyancy force, and under wall-normal-oriented and downstream-oriented gravitational acceleration.

The near-wall low-speed streaks in the fixed-property case may be visualized through iso-surfaces of the instantaneous streamwise perturbation velocity, $u^{\prime \prime}$, as illustrated in Figure 5-8(a) for the lower wall. The iso-surface level in this figure is selected to correspond to the peak value of the rms of $u^{\prime \prime}$. Figure 5-8(b) plots iso-surfaces of the wall-normal turbulent heat flux, $\left(v^{\prime \prime} T^{\prime}\right)^{+}=1 \times 10^{-4}$, where a positive value of $v^{\prime \prime}$ indicates fluid motion away from the wall. The plotted iso-surface value corresponds to the peak value of the mean of $\left(v^{\prime \prime} T^{\prime}\right)^{+}$in the heated-wall, fixed-property case. Kasagi \& Shikazono (1995) show that turbulent motions that lead to the preferential upwash of highertemperature fluid away from the heated wall (hot-fluid ejection), and/or downwash of lower-temperature fluid towards the heated wall (cold-fluid sweep) most significantly contribute to the production of wall-normal turbulent heat flux, and thus enhancement of heat transfer. Through comparison of the topologies of the iso-surfaces plotted in Figure 5-8(a) and (b), motions that generate the low-speed streaks are shown to preferentially eject higher-temperature fluid away from the wall. Likewise, the highspeed streaks were confirmed to coincide with the cold-fluid sweep regions in this figure.

The plots in Figure 5-9 facilitate a quantitative analysis of the streak topology observed in Figure 5-8. In Figure 5-9(a), several high- and low-speed streaks are shown at three spanwise/wall-normal planes via flood-plots of the streamwise perturbation velocity 


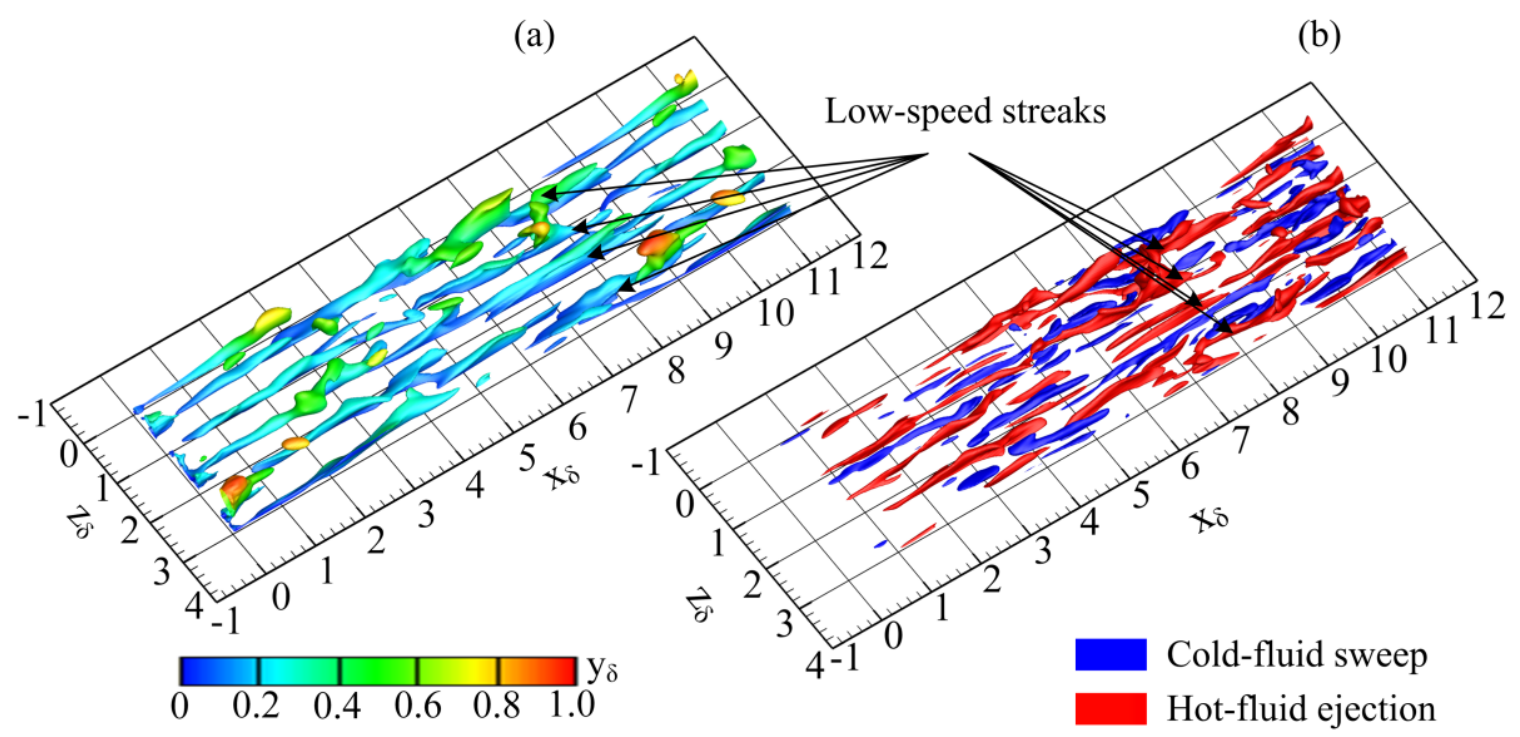

Figure 5-8: Streaks in the heated-wall, fixed-property case: a) low-speed streaks visualized through iso-surfaces of the instantaneous streamwise component of perturbation velocity $\left(u^{\prime \prime}=-0.3\right)$ coloured by wall-normal height; b) low- and high-speed streaks visualized through iso-surfaces of $\left(v^{\prime \prime} T\right)^{+}=1 \times 10^{-4}$.
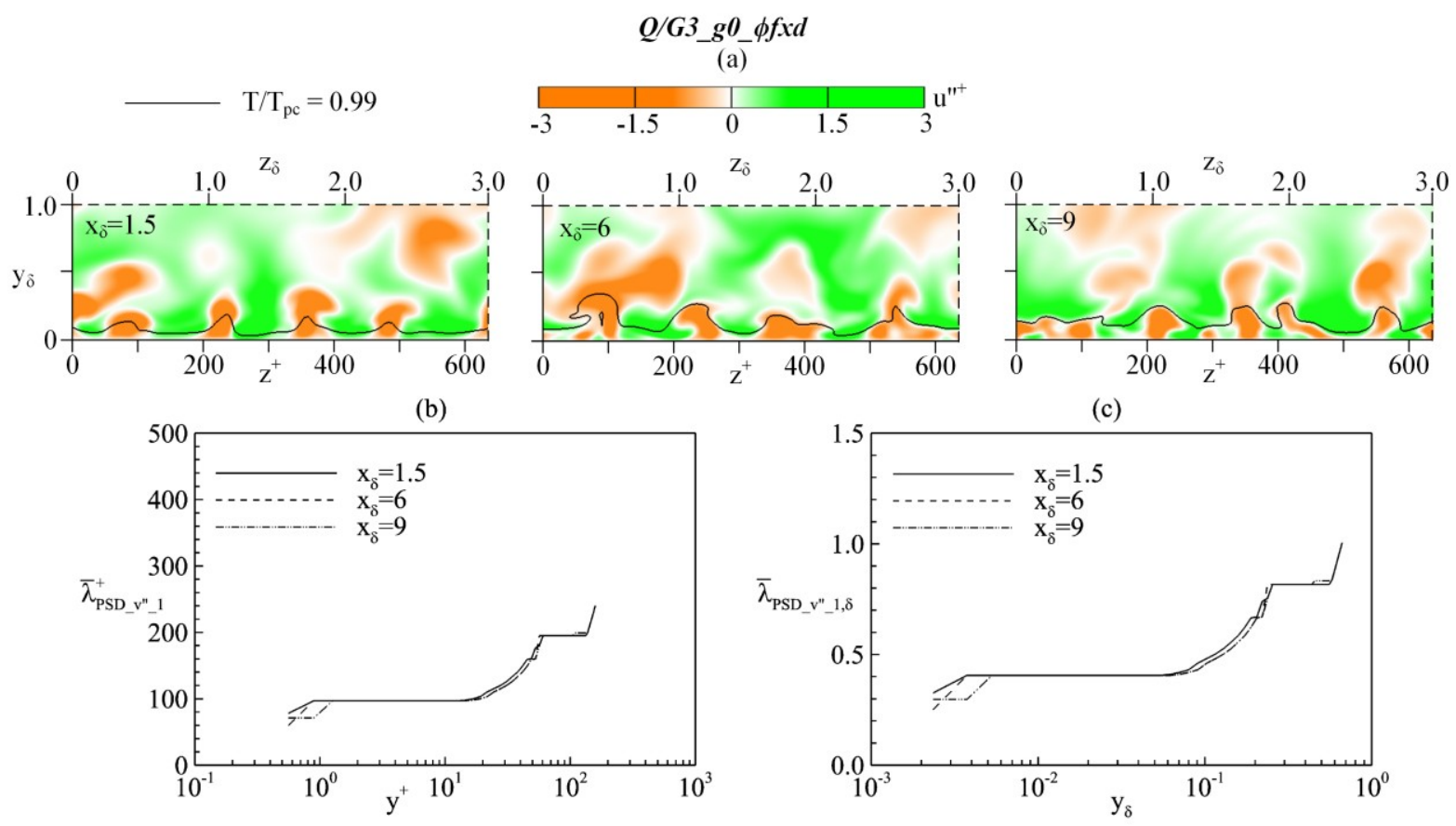

Figure 5-9: Velocity streaks in the heated-wall, fixed-property case identified by: a) flood-plots of the instantaneous streamwise component of perturbation velocity, $u^{\prime \prime} ; \mathrm{b}-\mathrm{c}$ ) wall-normal distribution of $\bar{\lambda}_{P S D_{-} v_{-}{ }_{1}}$, normalized by $\delta_{v}(\mathrm{~b})$ and $\delta(\mathrm{c})$. 
component, and with a "traffic-light" colour scheme that indicates low-speed streaks with amber colouring and high-speed streaks with green colouring. The range for the floodplots is selected to correspond to the peak value of the rms of $u^{\prime \prime}$. Complementing the observations in Figure 5-8, this solid-line contour illustrates that ejection motions typically result in higher-temperature fluid being ejected away from the wall and sweep motions typically result in lower-temperature fluid being transported towards the wall. To capture the distribution of length scales of the coherent flow structures more quantitatively, Figure 5-9(b,c) shows the wall-normal distribution of $\bar{\lambda}_{P S D_{-} v_{-}{ }_{1}}$. The region below a $y^{+}$value of 0.5 is omitted from the analysis, for the PSD values of $v^{\prime \prime}$ in this region are too small for the value of $\bar{\lambda}_{P S D_{-} v_{-}{ }_{-}}$to be informative. At a given time and wall-normal location, $\lambda_{P S D_{-} v_{-}-1}$ represents the spanwise wavelength of the strongest coherent flow structure. Plateau regions in the wall-normal profile of $\bar{\lambda}_{P S D_{-} v_{-}{ }_{-1}}$ indicate regions in which flow structures of certain sizes dominate. Figure 5-9(b) indicates the dominant flow structures to be near-wall and outer-region flow structures with mean spanwise wavelengths of $\Delta z^{+}=95 \pm 4$ and $195 \pm 10$, dominating the ranges $y^{+} \approx 1$ to 12 and $y^{+} \approx 70$ to 150 , respectively. Between these two plateaus, both the near-wall and outerregion flow structures have non-negligible influence on the flow field with the $\bar{\lambda}_{P S D_{-} v_{-}{ }_{1}}$ value reflecting the relative dominance of each flow structure. The fact that the spacing of the outer-region streaks is approximately twice that of the near-wall streaks suggests spanwise merging of near-wall vortical structures associated with the near-wall streaks as a plausible mechanism for the formation of the outer shear layer streaks, as per the 
description in Section 5.1 (Adrian et al., 2000; Tomkins \& Adrian, 2003; Brinkerhoff \& Yaras, 2014).

\subsubsection{Effects of Spatial Gradients in Thermophysical Properties on the Coherent Flow Structures of Turbulence in the Absence of Buoyancy Forces}

This section discusses the development of turbulence in a channel flow of supercritical water that is heated from a uniform inflow temperature of $T / T_{p c}=0.98$. The turbulence field introduced through the inflow boundary originates from a statistically steady turbulent flow field discussed in Sections 5.2.3. and 5.4.1. This inflow effectively represents an unheated upstream portion of the turbulent channel flow.

For constant property flows, it was noted in the previous section that the spanwise spacing between adjacent near-wall streaks and the associated hairpin vortices scales on the viscous length scale, expressed as:

$$
\delta_{v}=\frac{v}{u_{\tau}}=\left(\frac{v}{(\partial \bar{u} / \partial y)_{w}}\right)^{0.5}
$$

In Figure 5-10(a), the magnitude of the viscous length scale for the flow with variable thermophysical properties is observed to be notably smaller than with fixed thermophysical properties. As per Equation 5-9, the sharp decrease in the viscous length scale observed in the streamwise range of $x_{\delta}<0.5$, where $\bar{T}_{w}<T_{p c}$, occurs due to the increase in the strain rate at the wall while there is minimal variation of kinematic viscosity for this temperature range, as per Figure 5-11. The increase in strain rate is due to near-wall streamwise acceleration driven by local decreases in dynamic viscosity and 


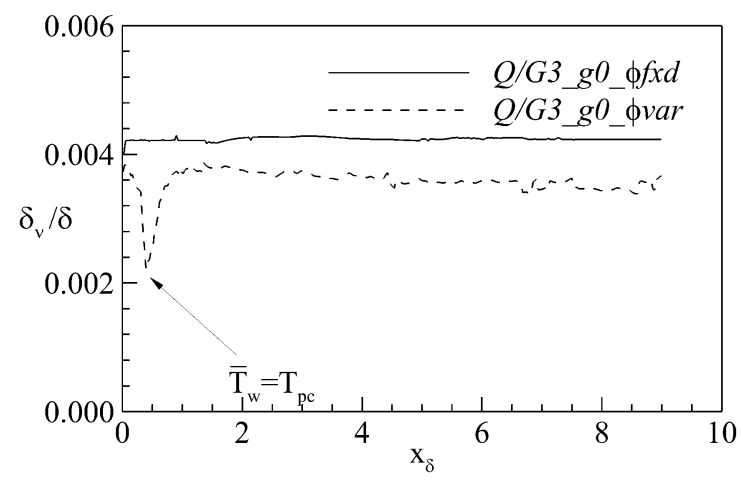

Figure 5-10: Comparison of streamwise distribution of the viscous length scale for flows with fixed and variable thermophysical properties in the absence of buoyancy forces.

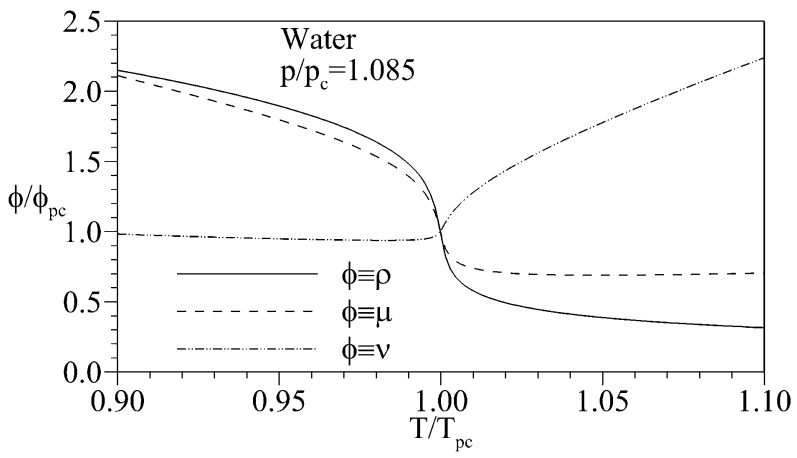

Figure 5-11: Temperature dependence of density, dynamic viscosity, and kinematic viscosity in the vicinity of the pseudo-critical state.

density with increasing wall temperature in the range of $T<T_{p c}$, also depicted in Figure 5-11. For $T>T_{p c}$ there is a notable increase in kinematic viscosity with increasing temperature, yielding the rapid increase in viscous length scale immediately downstream of $\bar{T}_{w}=T_{p c}$. The generation and growth of turbulence structures in this region of reduced viscous length scales is discussed next, followed by discussions of their interactions with the turbulence structures convecting from the unheated upstream flow. 


\subsubsection{Flow Development near the Inflow Plane}

Figure 5-12 plots iso-contours of the second invariant of the velocity gradient tensor, $q$, to visualize the wavepackets in the upstream region of the fixed-property and variableproperty cases. The value of $q$ plotted is selected to most vividly highlight the hairpin vortices of interest. Data in the region surrounding these clusters of flow structures has been removed to enhance clarity of the plots. Hairpin vortices in near-wall wavepackets that convect from the upstream domain are labelled with uppercase letters. In the variable-property case, these vortices interact with the wavepackets of vortices generated through the baroclinic mechanism, as will be described later on, which extend up to $y_{\delta}=0.1$ and are labelled with lowercase letters. This interaction generally results in some loss of coherence of the hairpin vortices in both of the mutually-interacting wavepackets. For example, in Figure 5-12(b) the encircled regions of the streamwise legs of the hairpin

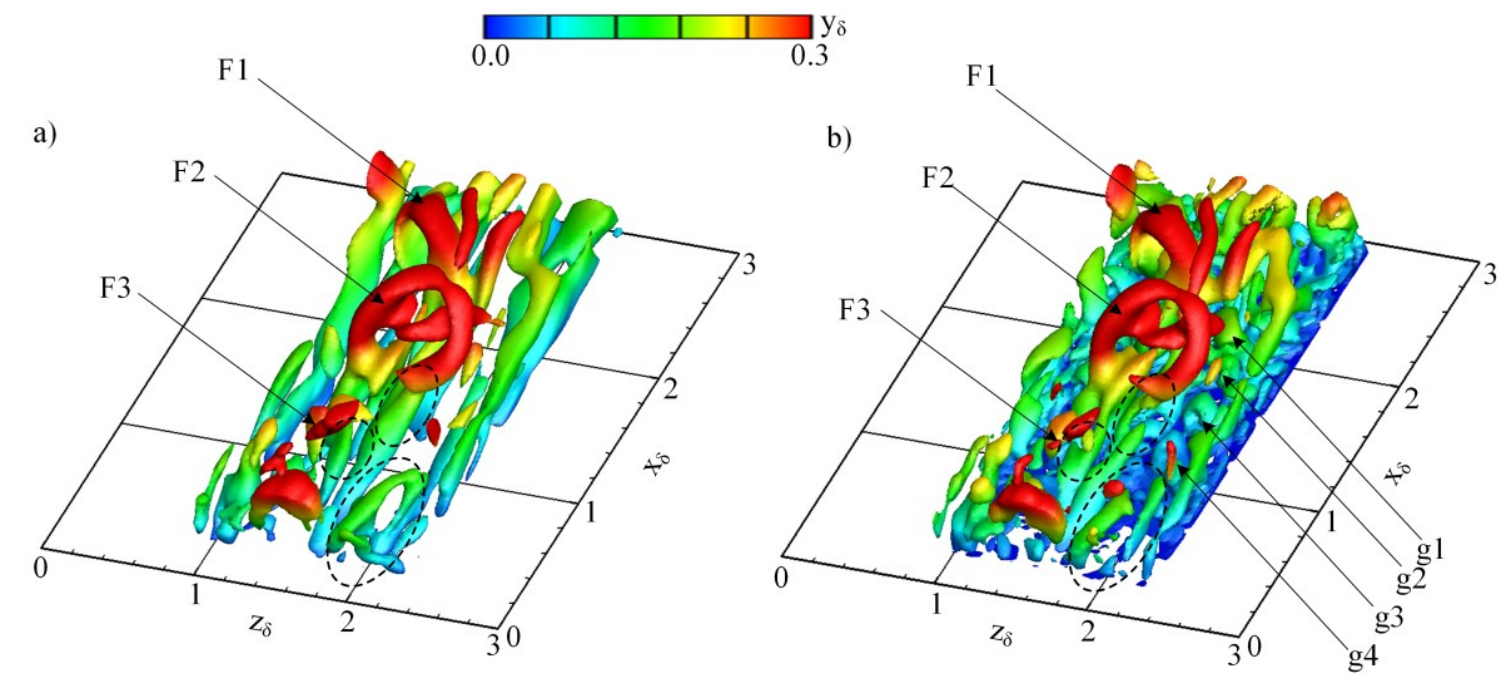

Figure 5-12: Iso-contours of the second invariant of the velocity gradient tensor $\left(q^{+}=0.01\right.$ based on inflow wall variables) coloured by wall-normal height for: a) heated-wall, fixed property case; b) non-buoyant, variable-property case. 
vortices labelled F2 and F3 are observed to be broken up by a smaller near-wall wavepacket emerging from underneath it. Likewise, the head of the encircled near-wall hairpin vortex located at $z_{\delta}=2$ and $x_{\delta}=0.8$, is broken up. To assist in visualizing the formation of the smaller near-wall wavepackets, Figure 5-13 illustrates the development of the flow in the region $0<x_{\delta}<1.5$, which is the region of reduced viscous length scales, at $z_{\delta}=1.7$. The fixed-property case is shown in part (a) of this figure for comparison, where spanwise vorticity with a clockwise sense of rotation is taken as negative. An outline of the wavepacket of hairpin vortices that convected from the upstream domain is superimposed on the vorticity field, as traced from the iso-contours of $q$ plotted in Figure 5-12. The wavepackets denoted by the $\mathrm{H}$ label in Figure 5-13 are not clearly visible in the iso-contour plots of Figure 5-12 as they as they are developing underneath the wavepackets of vortices denoted by the F label. The regions of more intense negative spanwise vorticity above these outlined vortices are consistent with the flow field around a hairpin vortex summarized in Figure 5-2, whereby such regions of negative shear are created when the ejection of low-speed fluid between the legs of the hairpin vortex encounters the faster moving fluid above the vortex. Notable differences between the vorticity fields of the two cases are apparent, beginning very close to the wall at the onset of heating of the fluid. In this region of the flow with variable thermophysical properties, illustrated in Figure 5-13 (b), positive vorticity is observed in close proximity to the wall. This vorticity is generated via the baroclinic mechanism which manifests when fluid particles are accelerated through a region with cross-stream density gradients. Such vorticity is oriented in the direction of the cross product of the acceleration vector, $\vec{a}$, and 
a)
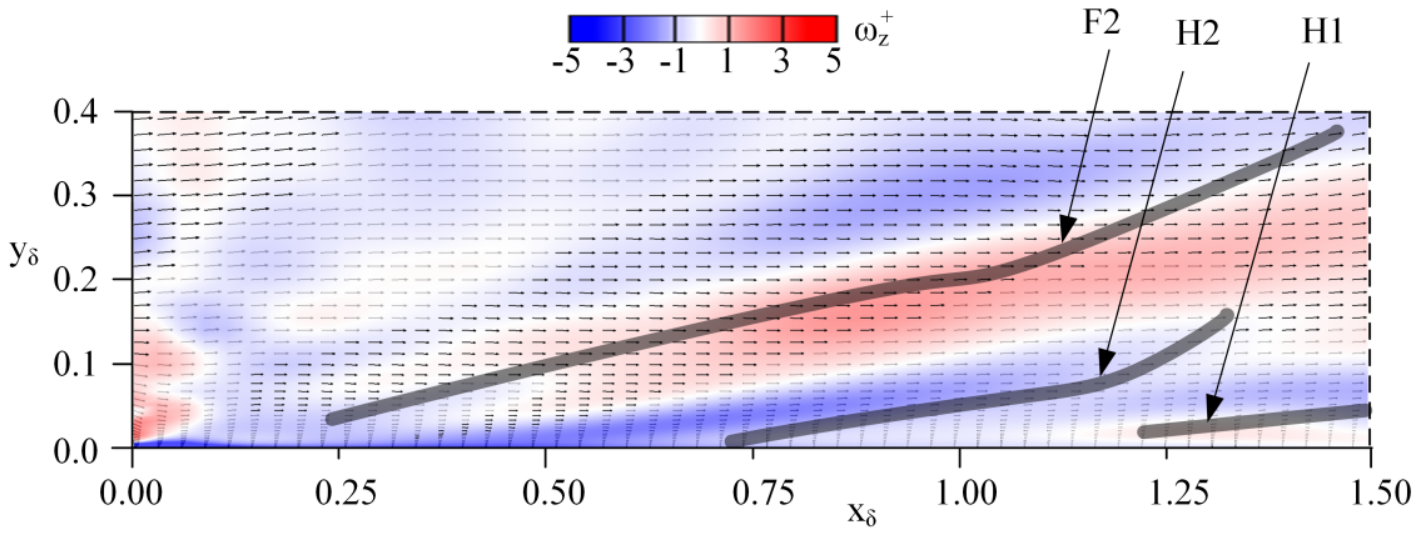

b)
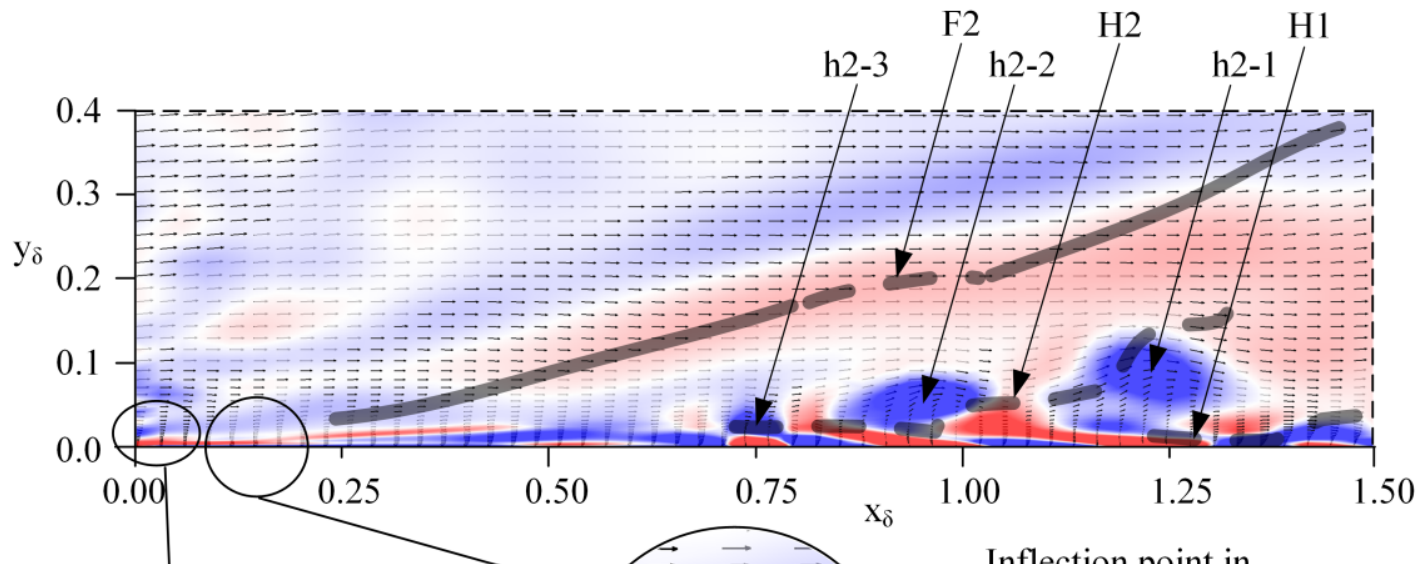

Figure 5-13: Flow development in the streamwise region where $\bar{T}_{w}$ is in the vicinity of the pseudo-critical temperature, observed through flood-plots of spanwise vorticity $\left(\omega_{z}\right)$ and velocity vectors in the: a) heated-wall, fixed-property case; b) non-buoyant, variableproperty case.

the density gradient, $\nabla \rho$, as schematically illustrated in one of the insets of Figure 5-13 (b) for fluid particles accelerated from point "A" to point "B" (Jiang \& Luo, 2000; Sreenivas \& Prasad, 2000). The velocity vectors in the magnified view of this near- 
wall region reveal notable streamwise acceleration of the near-wall fluid that yields the baroclinic vorticity. This acceleration is due to volumetric expansion of this fluid and reduced resistance to flow as per the temperature-induced decrease in density and dynamic viscosity. An illustration of the spatial distribution of density and dynamic viscosity is shown in Figure 5-14. The magnified image of the near-wall shear layer in Figure 5-13 (b) shows that generation of positive vorticity in close vicinity of the wall via the baroclinic mechanism imposes an inflection point on the velocity profile at the wallnormal location between this region of positive vorticity region and the region of negative vorticity father away from the wall. This velocity profile is inviscid unstable, which accelerates streamwise grouping of spanwise vorticity that rolls up to form hairpin vortices. The accelerated roll-up of the near-wall shear layer to form hairpin vortices is evident through the vortices labelled h2-1, h2-2, and h2-3 in Figure 5-13 (b) that roll-up from the negative vorticity shear layer above the $\mathrm{H} 2$ hairpin vortex. This observation is

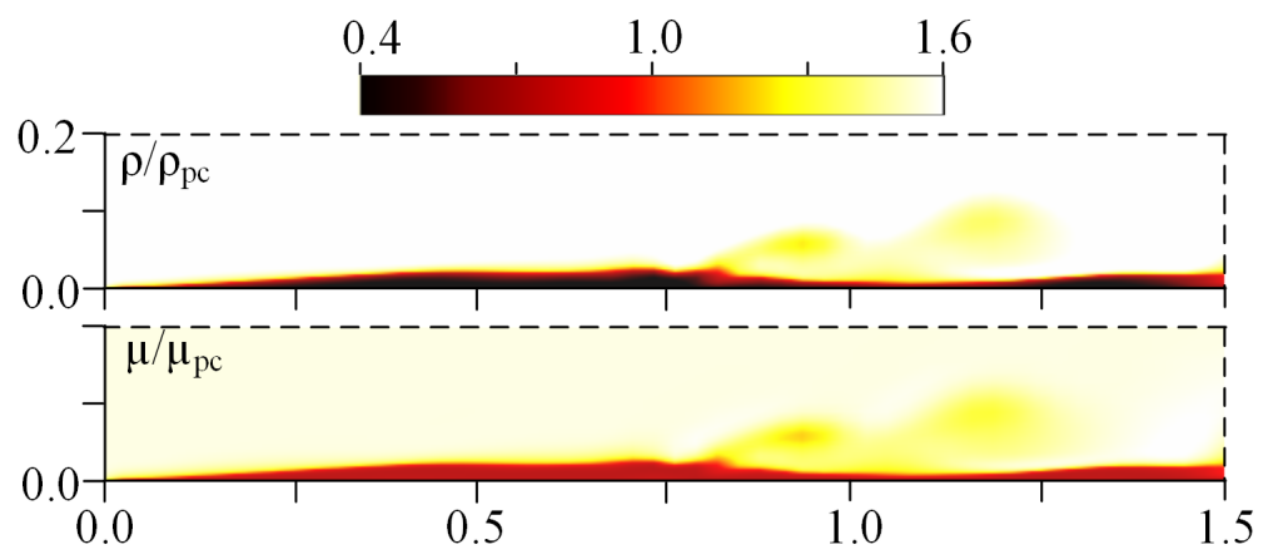

Figure 5-14: Flow development in the streamwise region where $\bar{T}_{w}$ is in the vicinity of the pseudo-critical temperature, observed through flood-plots of density and dynamic viscosity. 
consistent with those of Reinink \& Yaras (2015), who show that baroclinic vorticity generation in the vicinity of an inviscid-unstable shear layer accelerates the perturbation growth rate that leads to roll-up of the shear layer. As per Figure 5-14, the rolled-up vorticity transports lower-density, higher-temperature fluid farther away from the wall, thereby locally enhancing the wall-normal transfer of mass and thermal energy.

The flow dynamics may be further investigated by examining the budgets of vorticity, $\omega$, expressed by Equation 5-10:

$$
\frac{D \omega_{i}}{D t}=\underbrace{\omega_{j} \frac{\partial u_{i}}{\partial x_{j}}}_{P_{\omega, \text { stretc }}} \underbrace{-\omega_{i} \frac{\partial u_{j}}{\partial x_{j}}}_{P_{\omega, \text { expand }}}+\underbrace{\frac{\epsilon_{i j k}}{\rho^{2} \frac{\partial \rho}{\partial x_{j}} \frac{\partial p}{\partial x_{k}}}}_{P_{\omega, \text { baroclinic }}}+\underbrace{\epsilon_{i j k} \frac{\partial}{\partial x_{j}}\left(\frac{1}{\rho} \frac{\partial \tau_{k m}}{\partial x_{m}}\right)}_{P_{\omega, \text { viscous_diffusion }}} \text { 5-10 }
$$

The first term on the right-hand side represents local changes in the vorticity field due to stretching and tilting of vorticity. This term is known to dominate vortex dynamics in constant-property turbulent flows. In variable-density turbulent flows the local vorticity field may be significantly affected by local fluid expansion and local production of baroclinic torque represented by the second and third terms in Equation 5-10, respectively. The negative sign preceding $P_{\omega, \text { expand }}$ indicates that localized fluid expansion $\left(\partial u_{j} / \partial x_{j}>0\right)$ acts to decrease the local magnitude of vorticity. The ratio of the rms of the baroclinic torque and local expansion to that of the stretching/tilting term in the budget of the streamwise component of vorticity is plotted in Figure 5-15 at various streamwise locations. The streamwise component of vorticity is presented here as it dominantly contributes to ejection and sweep motions. The magnitudes of $\left(P_{\omega, \text { baroclinic }} / P_{\omega, \text { stretch }}\right)_{x}$ and $\left(P_{\omega, \text { expand }} / P_{\omega, \text { stretch }}\right)_{x}$ are notably higher than unity near the wall, which indicates that effects of baroclinic vorticity generation and local fluid expansion dominate those of 
vortex stretching and tilting on the local vorticity field. Indeed, in very close vicinity of the wall, effects of fluid expansion are observed to be most dominant especially in close proximity to the onset of heating at $x_{\delta}=0$. This effect manifests as a local reduction in the magnitude of streamwise vorticity with streamwise distance from the onset of heating and occurs through redistribution of local vorticity by thermal expansion of the fluid. However slightly farther away from the wall while remaining below $y_{\delta}=0.05$, baroclinic vorticity generation mechanisms dominate as also observed in Figure 5-13(b). Downstream of $x_{\delta}=1.5$, the near-wall effects of baroclinic vorticity generation and local fluid expansion on the local vorticity field become of comparable magnitudes. In this streamwise region, as the baroclinic-generated near-wall hairpin vortices convect downstream, they extend away from the wall due to self-induction. Eventually, these hairpin vortices begin to interact with older generations of near-wall hairpin vortices, which are created in the upstream domain and have convected to the same streamwise location. The following section present additional information on this interaction.

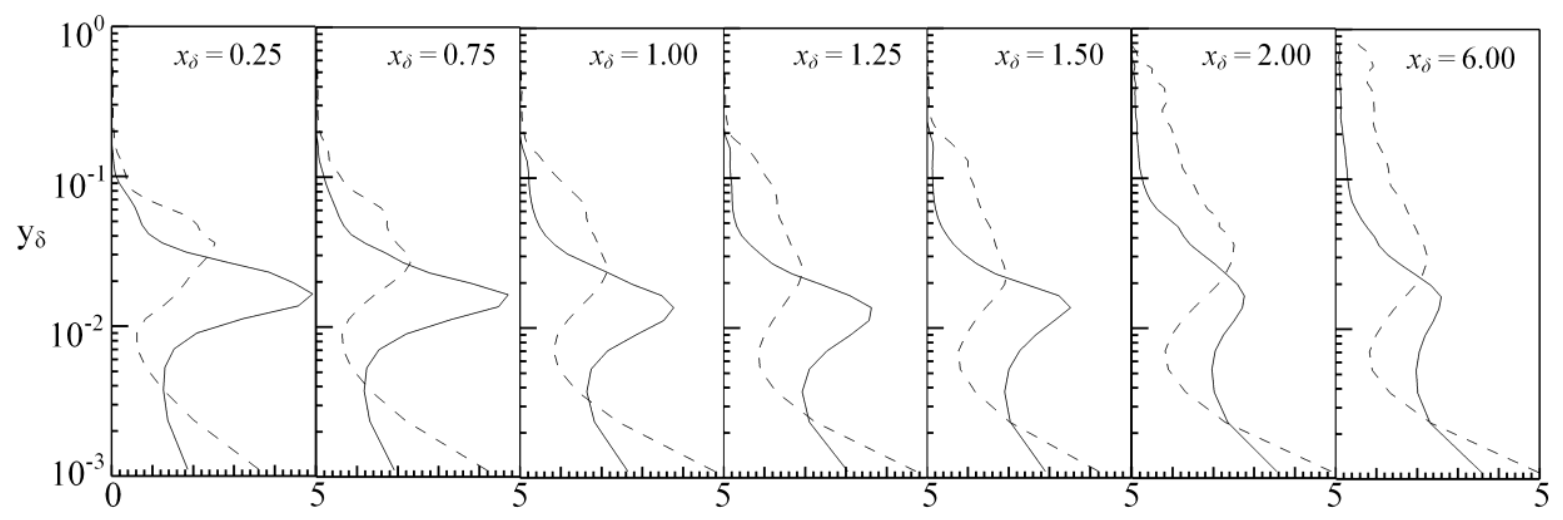

Figure 5-15: Ratio of the rms budgets of local rates of change of streamwise component of vorticity: $\left(P_{\omega, \text { baroclinic, rms }} / P_{\omega, \text { stretch, } r m s}\right)_{x}$ (solid line); $\left(P_{\omega, \text { expand, } r m s} / P_{\omega, \text { stretch, rms }}\right)_{x}$ (dashed line). 


\subsubsection{Interaction between Near-Wall Wavepackets}

Flood-plots of the streamwise component of perturbation velocity over a streamwise/spanwise plane are shown in Figure 5-16 with the low-speed streaks associated with hairpin vortices created in the unheated upstream domain labelled with uppercase letters. This figure further illustrates the breakdown of wavepackets of hairpin vortices into smaller scales, observed as the degradation of their associated streamwiseoriented streaks beginning before $x_{\delta}=1$. This degradation interrupts the self-sustenance of turbulence that occurs through the well-established mechanics where the shear layer surrounding such streaks, created by the ejection and sweep motions of wavepackets of hairpin vortices is inviscid-unstable, leading to grouping of vorticity to form subsequent hairpin vortices as discussed in Section 5.1. Thus for the variable- property case, nearwall wavepackets of streamwise-aligned hairpin vortices are rarely observed in the region of $x_{\delta}>4$ and the corresponding near-wall streaks induced by the hairpin vortices are no longer elongated in the streamwise direction. Although the lower- and higher-speed fluid regions downstream of $x_{\delta} \approx 1$ no longer bear the topology of spanwise-periodic and streamwise-elongated fluid regions, they are respectively identified as low- and highspeed streaks herein to maintain consistency in terminology. To assist in performing a more quantitative analysis, Figure 5-17 visualizes low- and high-speed streaks for the non-buoyant, variable-property case through profiles of $\bar{\lambda}_{P S D_{-} v_{-}{ }_{1}}$ normalized by the viscous length and velocity scales. The aforementioned destructive interaction between near-wall hairpin vortices is evident in these figures as a decrease in $\bar{\lambda}_{P S D_{-} v_{-}{ }_{1} 1}$ with increased wall-normal distance, observed between $5<y^{+}<50$ and $8<y^{+}<30$ at $x_{\delta}=1.5$ 
and $x_{\delta}=6$, respectively. The fluid dynamics that drives the regeneration of turbulence downstream of the region of destructive interactions is discussed next.
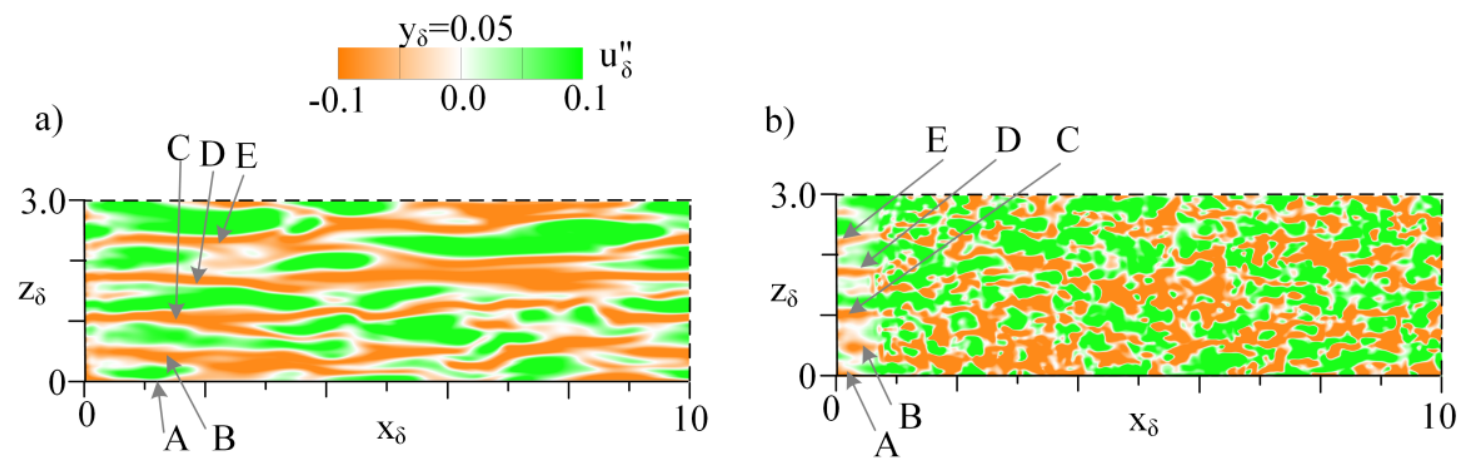

Figure 5-16. Flow development visualized through flood-plots of the streamwise component of perturbation velocity for: a) the heated-wall, fixed-property case; b) the non-buoyant, variable-property case.

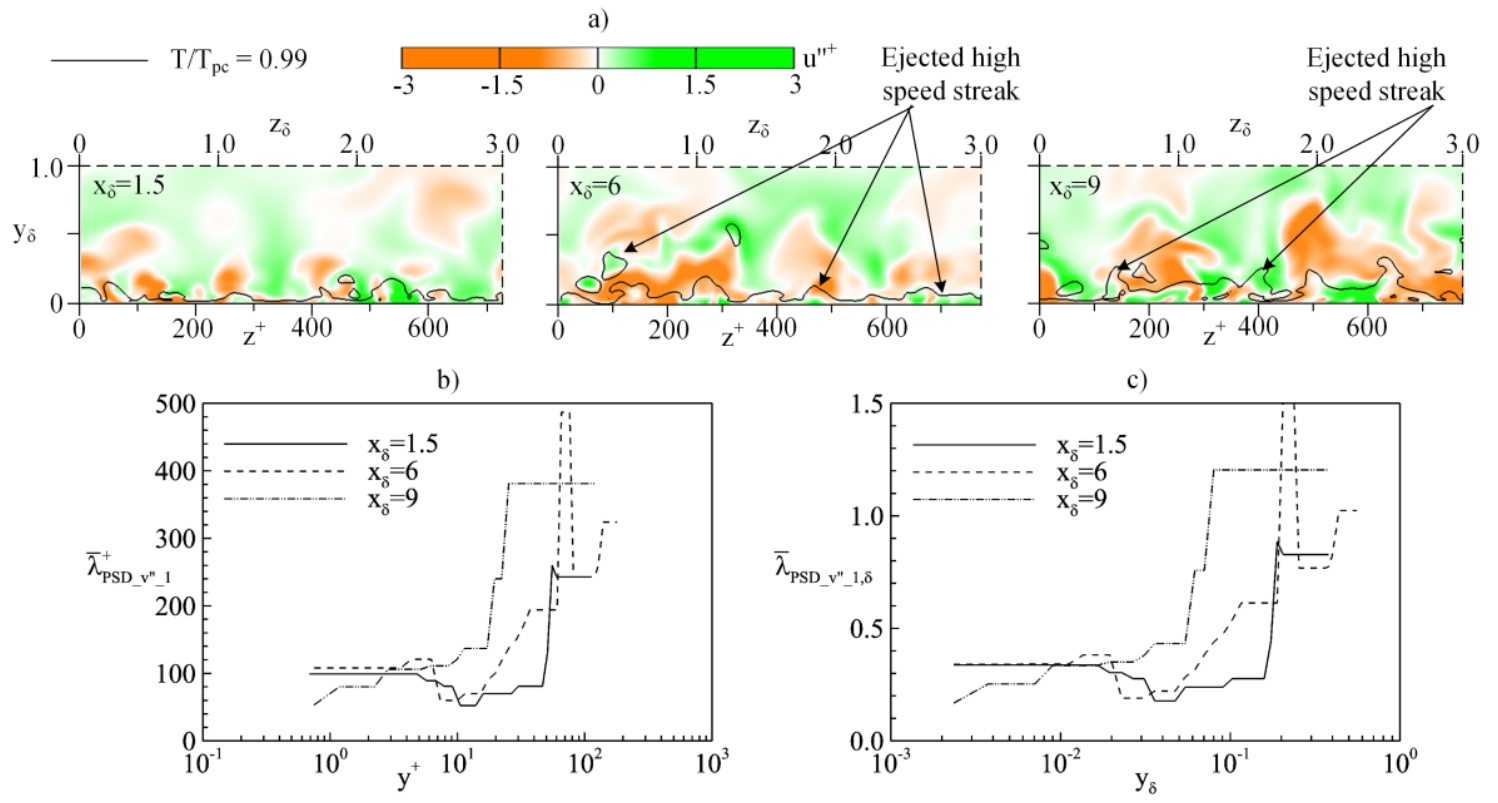

Figure 5-17: Streaky structures in the non-buoyant, variable-property case identified by: a) flood-plots of the instantaneous streamwise component of perturbation velocity, $u^{\prime \prime}$; bc) wall-normal distribution of $\bar{\lambda}_{P S D_{-} v^{\prime \prime}{ }_{-1}}$, normalized by $\delta_{v}(\mathrm{~b})$ and $\delta(\mathrm{c})$. 


\subsubsection{Regeneration of Organized Near-Wall Coherent Structures of Turbulence}

For the fixed-property case, it was noted through the temperature contours in Figure 5-9 that ejection motions typically result in higher-temperature low-speed streaks being ejected away from the wall and vice versa for sweep motions, and these motions have a spanwise wavelength of $\Delta z^{+}=95 \pm 4$ near the wall. The plateaus in the profile of $\bar{\lambda}_{P S D_{-} v_{-}{ }_{1}}$ for the variable property case, shown in Figure 5-17(b), indicate dominant near-wall flow structures with a mean spanwise wavelength of $\Delta z^{+} \approx 111 \pm 6\left(\Delta z_{\delta} \approx 0.35 \pm 0.02\right)$, which is similar to that of the fixed-property case. However, the temperature contours in Figure 5-17(a) indicate regions in which higher-temperature high-speed streaks are ejected away from the wall. These higher-temperature high-speed streaks will be demonstrated to be linked to populations of spanwise-aligned hairpin vortices, which dominate the near-wall flow in the region of $x_{\delta}>4$. Based on observations in more than 25 instances of the ejection motions that comprise of high-speed streaks being ejected away from the wall, the sequence of events leading to their formation is illustrated schematically in Figure 5-18. Prior to the ejection motion, a pocket of fluid having a bulk temperature less than the pseudo-critical temperature is induced towards the wall by downwash on the outboard side of the legs of a hairpin vortex; this forms a high-density high-speed streak shown in Figure 5-18(a). If this high-speed streak is sufficiently close to the wall, through the effect of heating at the wall its local temperature may reach or exceed the pseudo-critical, $T_{p c}$, temperature (Figure 5-18(b)). The acceleration that results from the decrease of density with increased temperature is primarily in the streamwise and wall-normal directions. As a consequence, the strain rate of the shear layer that envelops the high-speed streak increases, thereby intensifying the streamwise and wall- 
normal components of vorticity in this shear layer. The wall-normal vorticity shear layers are unstable via an inviscid instability mechanism, producing streamwise grouping of wall-normal vorticity near the downstream end of the streak (Brinkerhoff \& Yaras, 2014). Above this streak, an inflectional velocity profile is generated in a manner similar to that discussed for the region near the inflow plane in Section 5.4.2.1, promoting streamwise grouping of spanwise vorticity. This grouping of vorticity eventually yields a hairpin vortex that envelops the high-speed streak as shown in Figure 5-18(c). The ensuing upwash between the legs of the newly created hairpin vortex ejects the highertemperature high-speed streak away from the wall. As illustrated in Figure 5-18(d-f), the

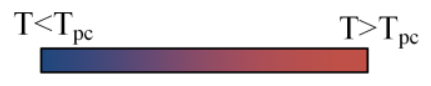

a)

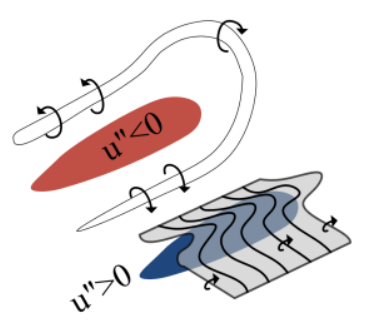

d)

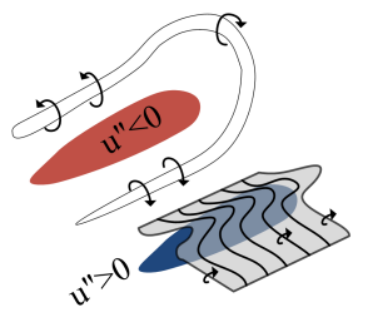

b)

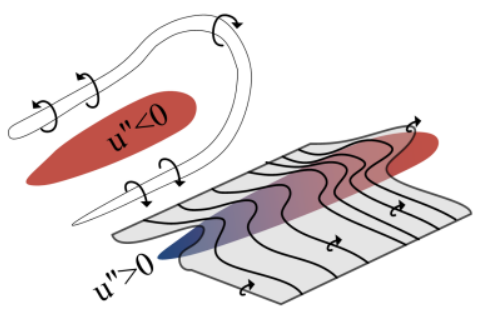

e)

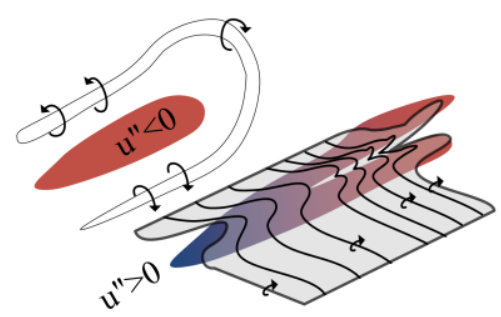

c)

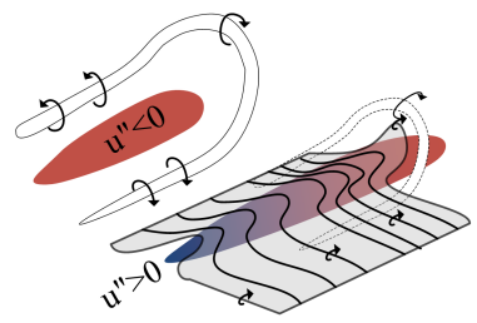

f)

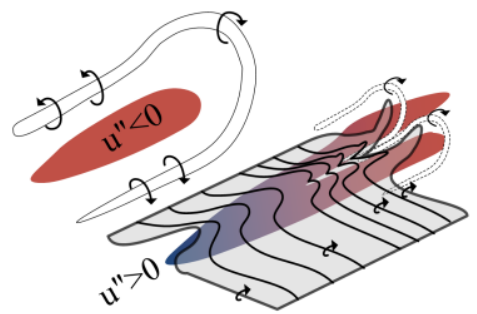

Figure 5-18. Illustration of the generation of high-speed fluid ejection motions along the wall of the non-buoyant, variable-property case: a-c) formation of a single hairpin vortex enveloping a high-speed streak; $\mathrm{d}-\mathrm{f}$ ) formation of a pair of hairpin vortices enveloping the downstream end of a high-speed streak. 
acceleration at the downstream end of the high-speed streak may occur in a spatially nonuniform manner such that two distinct regions of high-speed flow coexist at the downstream end of the streak. In such instances, a pair of hairpin vortices is formed which eventually merge to form a single hairpin vortex enveloping the downstream end of the high-speed streak. The flow development depicted in Figure 5-18 is similar to the mechanism that drives the lateral growth of a turbulent spot developing over a flat wall in the absence of thermal effects described by Brinkerhoff \& Yaras (2014).

The fluid dynamics leading to the ejection of high-speed fluid away from the wall is visible in the instantaneous streamwise velocity and wall-normal vorticity $\left(\omega_{y}\right)$ fields displayed in Figure 5-19. The range of $\omega_{y}$ in the flood-plots is selected to highlight the flow features of interest. This figure is generated using data from the lower channel wall of the case with wall-normal gravitational acceleration, and it will be shown later that the buoyancy force in that case act to intensify the coherent motions that prevail when the flow develops in the absence this force, thus enhancing the visibility of the underlying flow dynamics. A high-speed streak induced by an existing near-wall hairpin vortex is visible near the wall and is labelled $\mathrm{A}$ in Figure 5-19 at $t^{+}=0$ and $z^{+} \approx 850$. In this figure, $t=0$ is taken as the instance when the high-speed streak of interest forms near the wall and $t_{\delta}$ is time normalized by $\delta /\langle u\rangle$. This high-speed streak develops via the mechanism illustrated in Figure 5-18(d-f). The pair of near-wall hairpin vortices that develop at the downstream end of the high-speed streak are visible in Figure 5-19(b) as near-wall alternating regions of negative and positive wall-normal vorticity. The $-\omega_{y}$ legs of each 
these hairpin vortices are respectively labelled a1 and a2 in Figure 5-19(b) at $t^{+}=13$. By $t^{+}=22$, these near-wall hairpin vortices begin to interact, merging to form a single hairpin vortex of larger spanwise extent. Once this hairpin vortex is sufficiently mature, the upwash between its legs ejects the high-speed fluid in the streak farther away from the wall. An example of this ejection is labelled A at $t^{+}=32$ in Figure 5-19. At $x_{\delta}=9$, largerscale structures dominate the flow in the region $y^{+}>30\left(y_{\delta}>0.09\right)$ as illustrated in Figure 5-17(b,c). This wall-normal region of dominance for the larger-scale structures is greater than that for the fixed-property case of $y^{+}>70 \quad\left(y_{\delta}>0.3\right)$ illustrated in Figure 5-9(b,c). The greater wall- normal region of dominance may prevail at this streamwise location because the regeneration of near-wall coherent structures of

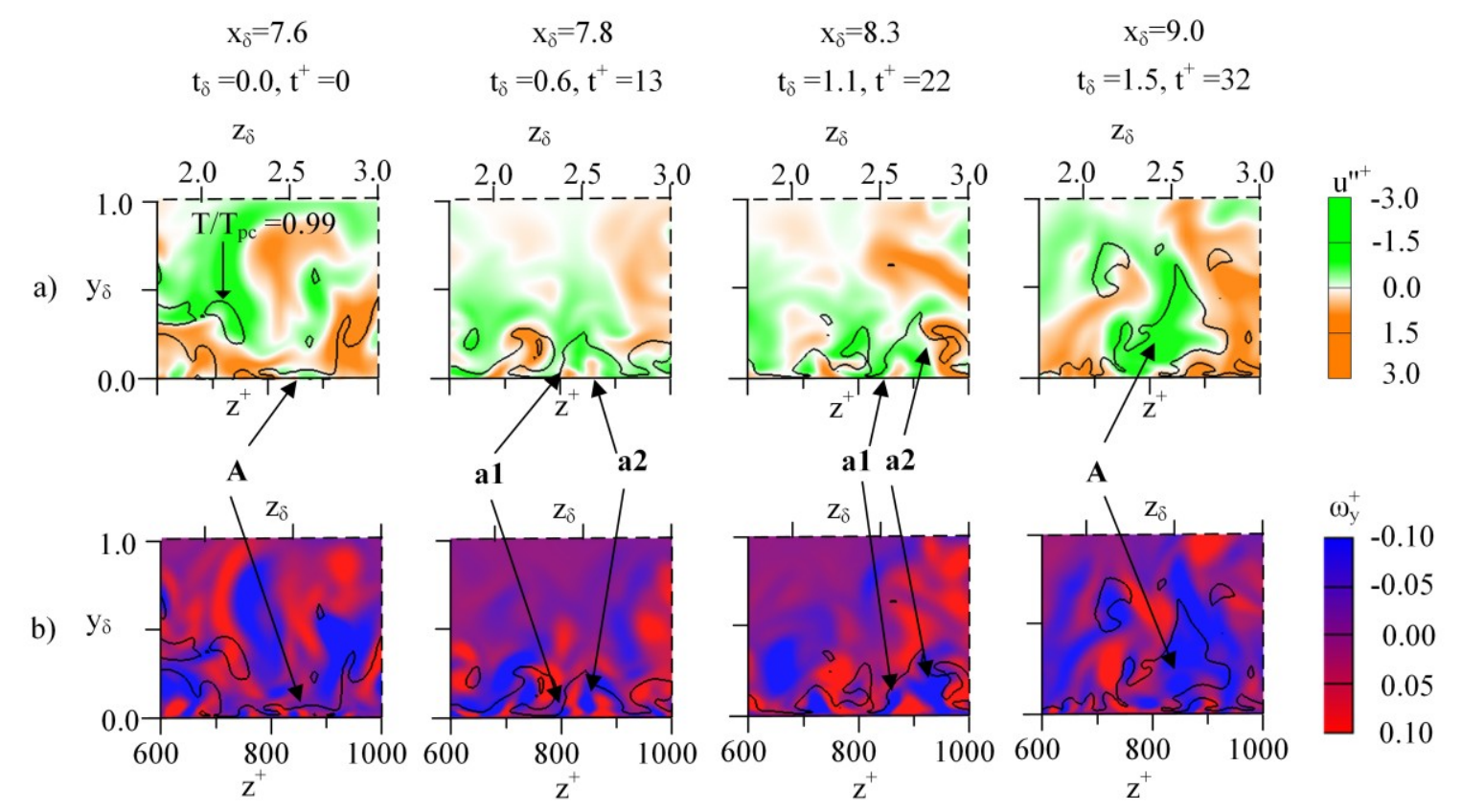

Figure 5-19. Instantaneous velocity and vorticity flood-plots for the lower wall of the case with wall-normal gravitational acceleration: a) low- and high-speed streaks visualized through flood-plots of instantaneous streamwise perturbation velocity component; b) flood-plots of instantaneous wall-normal component of vorticity. 
turbulence is still confined to $y^{+}<30$. It is likely that given sufficient streamwise development of the flow downstream of the region in which loss in coherence of nearwall hairpin vortices occurred, a turbulent state would be recovered whereby generations of wavepackets of streamwise-aligned self-replicating hairpin vortices dominate the flow.

\subsubsection{Deterioration and Enhancement of Heat Transfer}

Although it is recognized that the momentum and temperature fields are strongly coupled in the variable-property cases of the present study, particular focus is placed on the temperature field in this section to highlight the manner in which the foregoing discussions on the flow dynamics promote deterioration or enhancement of heat transfer. Figure 5-20, illustrates the wall-normal profile of the wall-normal temperature gradient at $x_{\delta}=9$ for the non-buoyant cases. Here, $T_{\delta}$ indicates temperature normalized by the mean centerline temperature. In place of the Nusselt number, the local wall-normal temperature gradient is chosen as an indicator of the wall-normal heat transfer rate because it better facilitates the understanding of localized heat transfer variations that are tied to changes in the development of coherent flow structures of turbulence within the shear layer. In the variable-property case, regions are observed where the wall-normal temperature gradient has values that exceed that of the fixed-property case, and other regions where the opposite is true. Heat transfer from the wall to the fluid layer adjacent to the wall occurs through thermal conduction, governed by Fourier's law of heat conduction. As expected, the case with variable thermophysical properties has a higher wall-normal temperature gradient at the wall due to the relatively lower values of thermal conductivity at the wall, as per Figure 5-1. With increasing distance from the wall, thermal mixing induced by the 
near-wall hairpin vortices in the region of turbulence regeneration causes the temperature gradient to reduce at a rate such that the wall-normal temperature gradient is lower than that of the heated-wall, fixed-property case in this region. The lack of streamwise elongation of these iso-surfaces of $\left(v^{\prime \prime} T^{\prime}\right)^{+}$, in Figure 5-21 when compared to the fixedproperty case is expected due to the aforementioned destructive interaction between near-
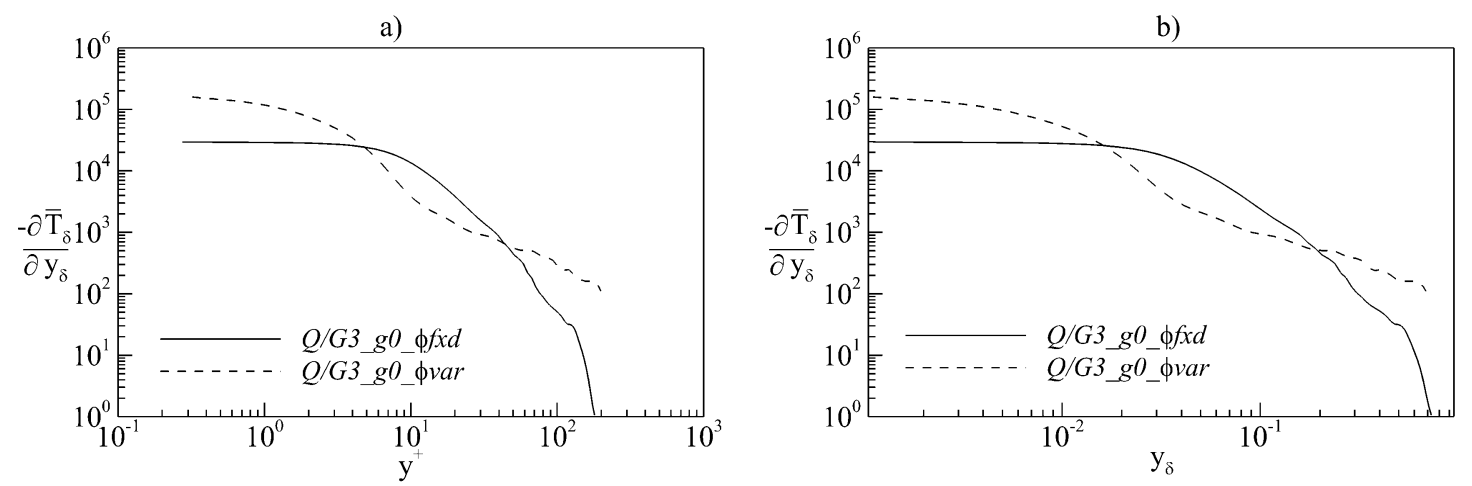

Figure 5-20. Wall-normal profile of the wall-normal temperature gradient for the nonbuoyant cases at $x_{\delta}=9$ plotted against: a) $y^{+}$; b) $y_{\delta}$.

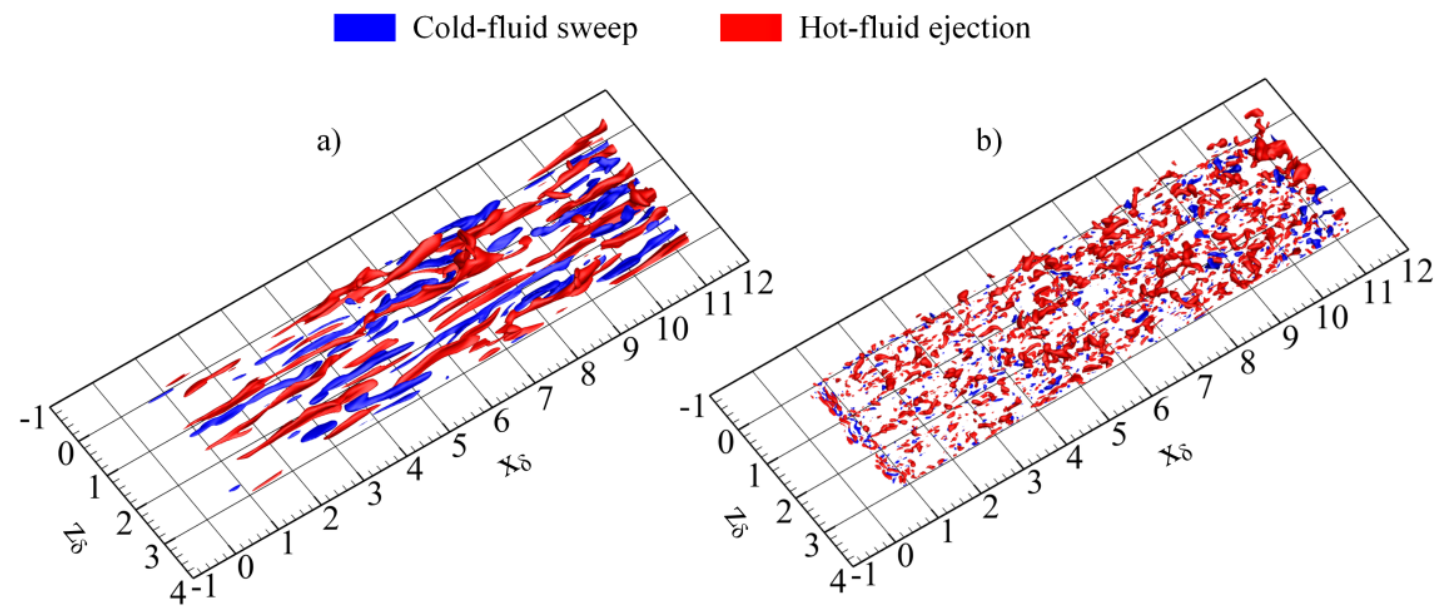

Figure 5-21. Iso-surfaces of $\left(v^{\prime \prime} T\right)^{+}=1 \times 10^{-4}$ for: a) the heated-wall, fixed property case; b) non-buoyant, variable-property case. 
wall hairpin vortices leading to the absence of wavepackets of streamwise-aligned hairpin vortices, as per the discussion in Section 5.1

\subsubsection{Effect of Spatial Gradients in Thermophysical Properties on the Coherent Structures of Turbulence in the Presence of Buoyancy Forces}

This section discusses the mechanisms through which a prevailing buoyancy force affects the fluid dynamics and heat transfer discussed for the non-buoyant, variable-property case in Section 5.4.2. The flow development in three scenarios is investigated, namely: along the lower channel wall of the case with wall-normal gravitational acceleration; along the upper channel wall of the case with wall-normal gravitational acceleration; and, along the walls of the case with downstream-oriented gravitational acceleration.

\subsubsection{Flow Development along the Lower Wall of the Case with Wall-Normal Gravitational Acceleration}

Figure 5-22 shows the streamwise distribution of the viscous length scale for the fixedproperty case, the non-buoyant, variable-property case, and the lower wall of the case with wall-normal gravitational acceleration. The minimum values of the viscous length scale for the latter two cases are noted to be close in value. Downstream of this minimum, the viscous length scale for the buoyant case rises to a magnitude that is $28 \%$ lower than the value for the non-buoyant, variable-property case. As will be shown, the buoyancy force enhances the wall-normal mixing due to turbulence, which yields fuller profiles of time-mean streamwise velocity, thus resulting in higher wall values of streamwise 


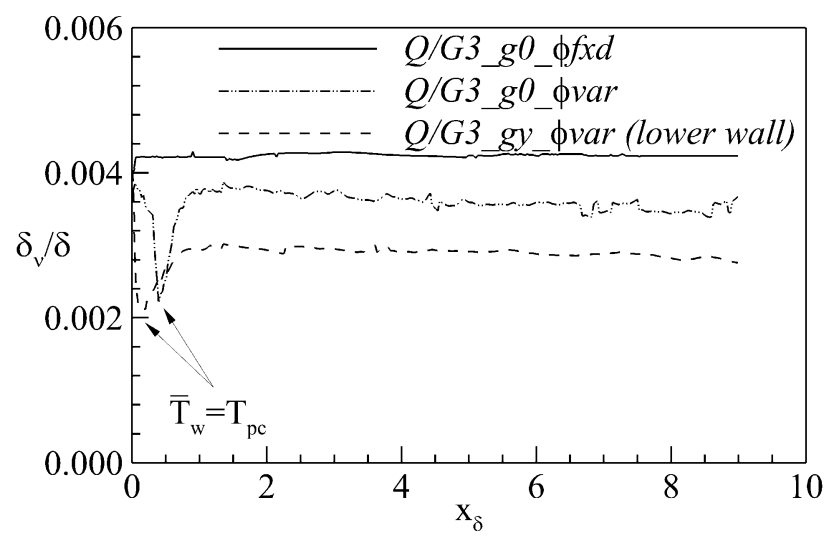

Figure 5-22: Streamwise distribution of the normalized viscous length scale for the heated-wall, fixed-property case, non-buoyant, variable-property case, and the lower wall of the case with wall-normal gravitational acceleration.

velocity gradients, and hence lower viscous length scales. In Figure 5-23(a), the high- and low-speed streaks prevailing at $1.5 \delta$ streamwise distance from the onset of wall heating are observed to develop in a manner similar to those described in Section 5.4.2. for the non-buoyant, variable-property case. The near-wall streaks created in this region have a mean spanwise spacing of $\Delta z^{+}=95 \pm 8\left(\Delta z_{\delta}=0.25 \pm 0.02\right)$. Along with a narrower spanwise spacing, these streaks are observed to have higher streamwise perturbation velocity magnitudes than those in the non-buoyant, variable-property case, as indicated by the relatively darker amber colouring of the low-speed streaks at $x_{\delta}=1.5$ in Figure 5-23(a) compared to those in Figure 5-17(a). The elevated magnitudes of streamwise perturbation velocity originate from generation of vorticity via the baroclinic mechanism which is visualized in Figure 5-24 through a schematic of the topology of streaks that form around a hairpin vortex. This schematic is constructed through observations of over 20 instances of such vortices, and the streaks are coloured by the sign of wall-normal perturbation velocity component so that the schematic illustrates both conventional hairpin vortices 


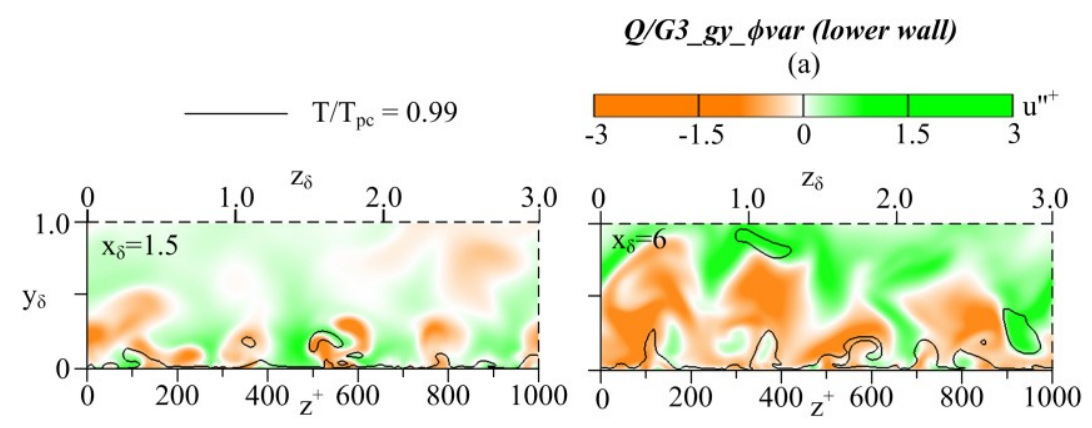

(b)

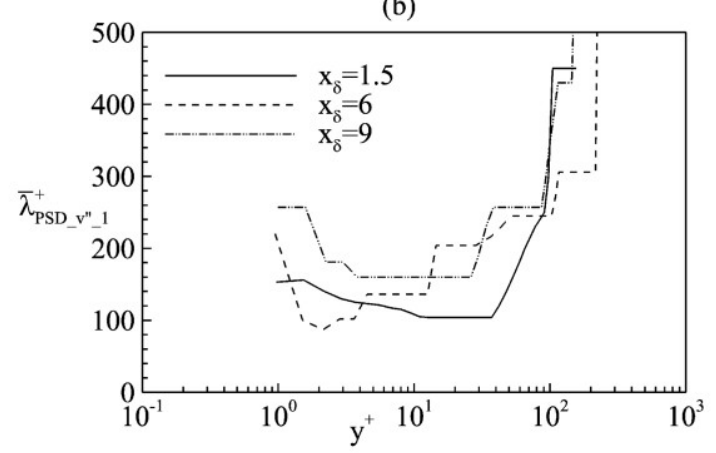

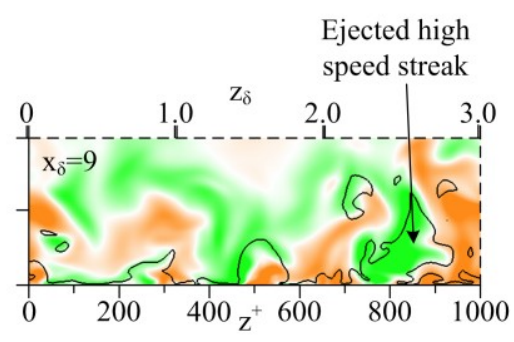

(c)

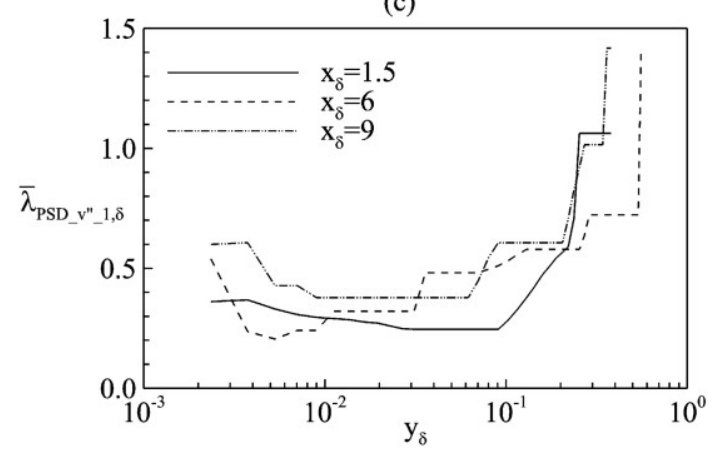

Figure 5-23: Velocity streaks along the lower wall of the channel in the presence of wallnormal gravitational acceleration identified by: a) flood-plots of the instantaneous streamwise component of perturbation velocity, $u^{\prime \prime}$; b-c) wall-normal distribution of $\bar{\lambda}_{P S D_{-} v_{-}{ }_{1}}$, normalized by $\delta_{v}(\mathrm{~b})$ and $\delta(\mathrm{c})$.

described in Section 5.4.1 and hairpin vortices that eject high-speed streaks as described in Section 5.4.2.3. The orientation of the gravitational acceleration vector is such that lower-density, higher-temperature fluid regions have a net buoyancy force in the $+y$ direction. Thus, higher-temperature streaks that form due to ejection between the legs of the hairpin vortices are affected by a net buoyancy force in the $+y$ direction. This flow topology is visualized through the density field in Figure 5-25 where the hairpin vortices appear in the form of small islands of low density fluid surrounded by regions of higher density fluid. Figure 5-24 highlights the generation of streamwise vorticity through the baroclinic mechanism (Figure 5-13(b)) driven by the wall-normal acceleration of these 


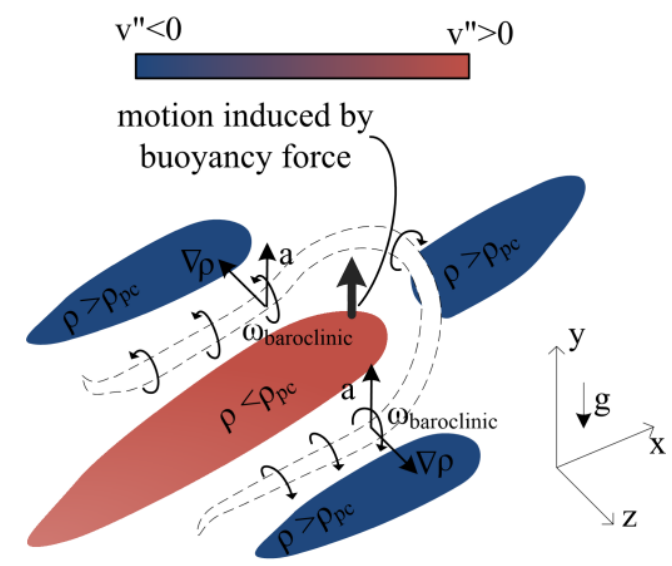

Figure 5-24: Illustration of the generation of vorticity through the baroclinic instability mechanism along the lower channel wall.

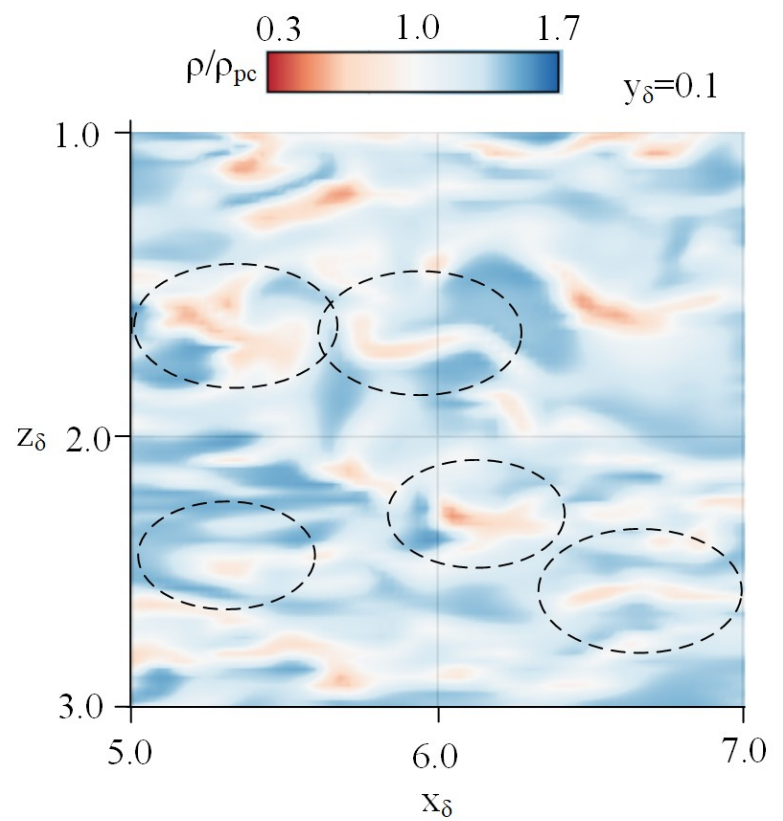

Figure 5-25: Flood-plots of density near the lower wall where the encircled regions are areas occupied by hairpin vortices.

streaks due to the buoyancy force as per spanwise density gradients prevailing near the legs of the hairpin vortex. Consequently, the streamwise component of vorticity in the legs of the hairpin vortices is more intense in comparison to that of the non- buoyant, variable-property case. The more intense streamwise vorticity, in turn, yields ejection 
motions of higher intensity. Notably, the ejected high-speed streaks, described for the non-buoyant, variable property case as per Figure 5-18 propagate to higher wall-normal distances in the present case as highlighted at $x_{\delta}=9$ in Fig. 16(a) compared to those in Figure 5-17(a).

With reference to Equation 5-10, Figure 5-26 shows wall-normal profiles of $\left(P_{\omega, \text { baroclinic,rms }} / P_{\omega, \text { stretch,rms }}\right)_{x}$ and $\left(P_{\omega, \text { expand,rms }} / P_{\omega, \text { stretch,rms }}\right)_{x}$ for the lower wall of the case with wall-normal gravitational acceleration and for the non-buoyant variable-property case. The baroclinic torque and local expansion of the fluid dominate vortex stretching/tilting in the near-wall region of both cases. Between the two cases, the relative contribution of the baroclinic torque is observed to be more dominant near the lower wall of the case with wall-normal gravitational acceleration. Flood-plots of local instantaneous streamwise vorticity production through the baroclinic mechanism are shown in Figure 5-27 for both cases along with contours of positive wall-normal perturbation velocity. The levels of the floods and contours are respectively chosen to highlight the regions where the magnitudes of these parameters are highest. Along the lower wall of the case with wall-normal oriented vorticity, the strongest ejection events are bordered by positive production of streamwise vorticity on the side with larger values of $+z$ and vice versa on the opposite side. This topology is consistent with the discussion of the schematic illustration of the hairpin vortex in Figure 5-24. In the case without buoyancy forces, the foregoing topology of fluid ejection and the related baroclinic vorticity production does not appear to be as prominent. 

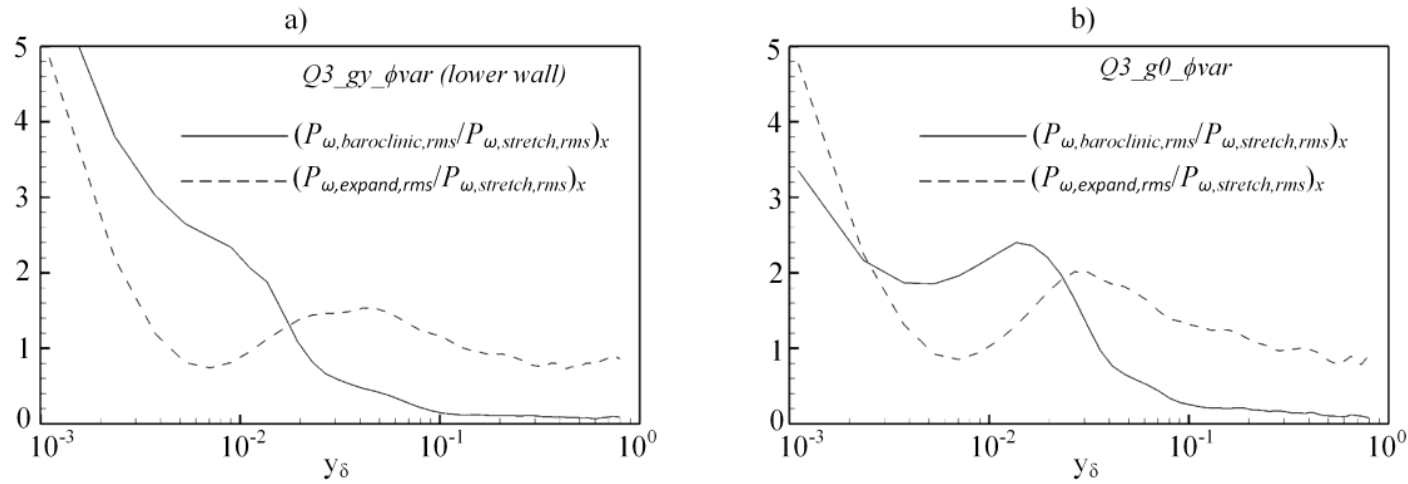

Figure 5-26. Ratio of the rms budgets of local rates of change of streamwise component of vorticity at $x_{\delta}=9$ : a) Lower wall of the case with wall-normal gravitational acceleration; b) the non-buoyant, variable-property case.

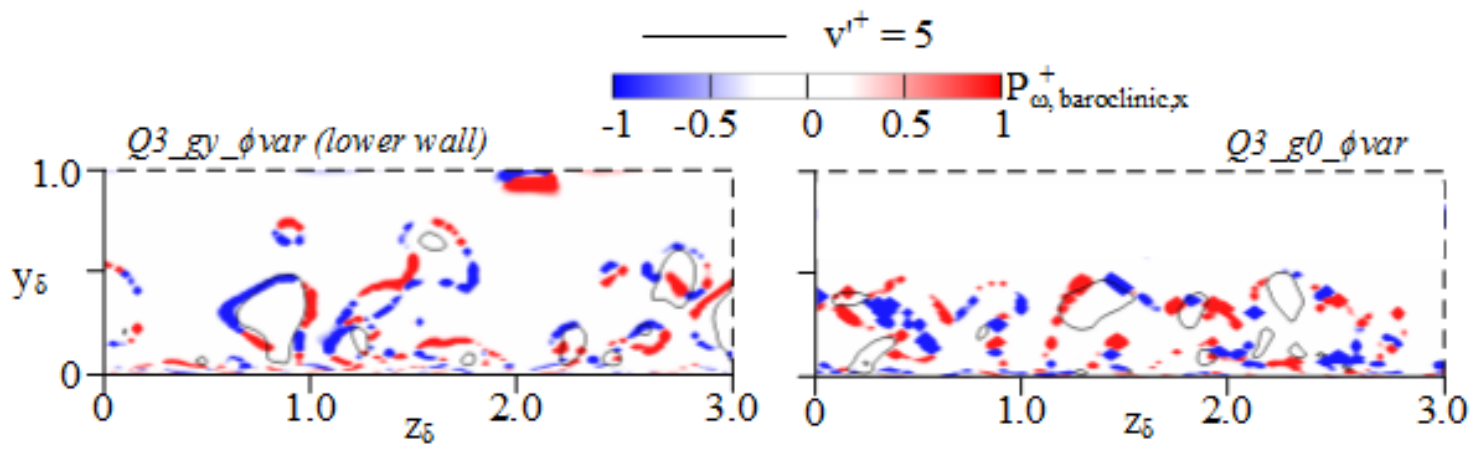

Figure 5-27. Flood plots of the production of vorticity via the baroclinic mechanism at $x_{\delta}=9$ for the lower wall of the case with wall-normal gravitational acceleration and for the non-buoyant variable-property case.

As per the more intense ejection motions of higher-temperature fluid driven by the mechanism described in the foregoing, the iso-surfaces of $\left(v^{\prime \prime} T^{\prime}\right)^{+}=1 \times 10^{-4}$ for the lower wall of the case with wall-normal gravitational acceleration, shown in Figure 5-28, indicate larger regions of hot-fluid ejections. In comparison to the non-buoyant, variableproperty case, these motions further enhance heat transfer, and thus yield lower values of the wall-normal temperature gradients as illustrated in Figure 5-29. This observation 


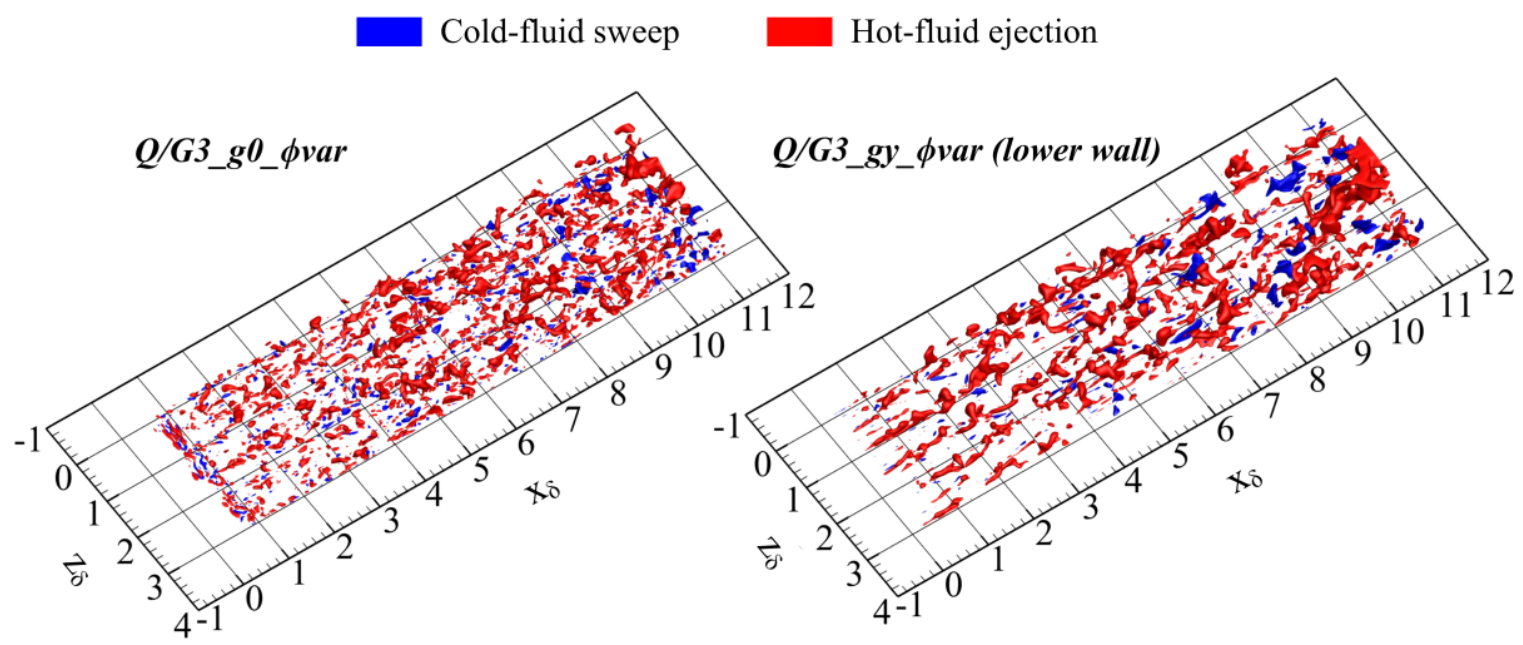

Figure 5-28: Iso-surfaces of $\left(v^{\prime \prime} T\right)^{+}=1 \times 10^{-4}$ for: a) the non-buoyant, variable-property case; b) the lower wall of the case with wall-normal gravitational acceleration.
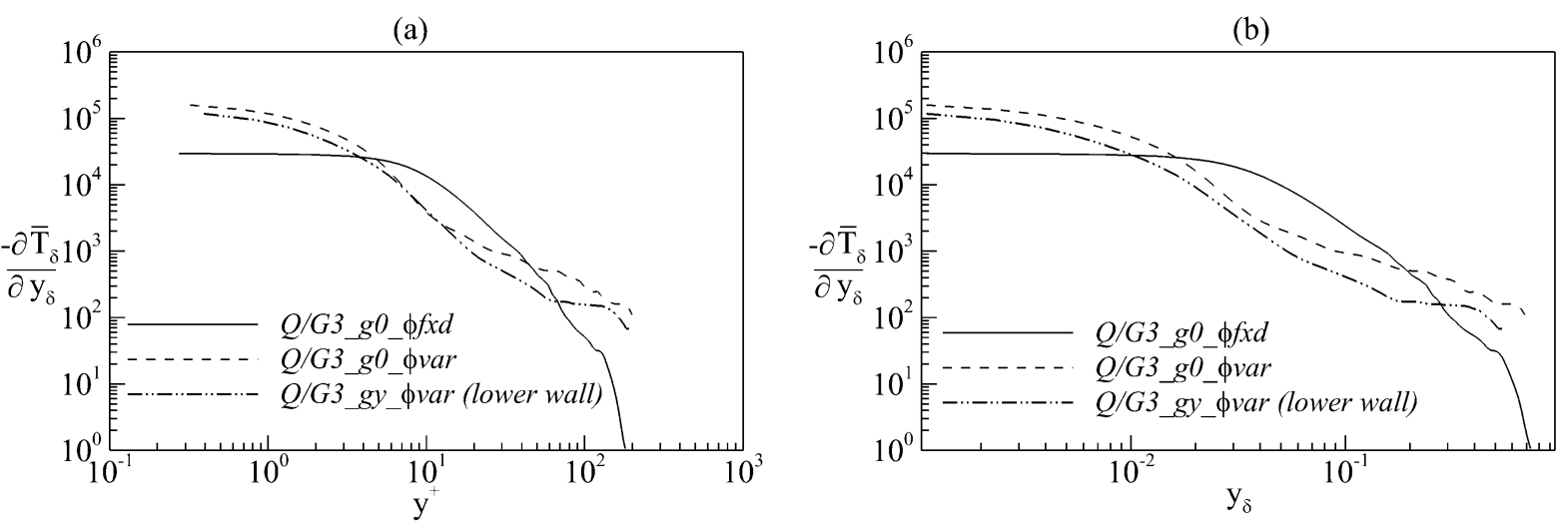

Figure 5-29: Wall-normal profile of the wall-normal temperature gradient for the nonbuoyant cases, and the lower wall of the case with wall-normal gravitational acceleration at $x_{\delta}=9$ plotted against: a) $y^{+}$; b) $y_{\delta}$.

indicates that the net effect of buoyancy-driven instabilities, under the present flow conditions with wall-normal gravitational acceleration, is an enhancement of heat transfer from the lower-wall of the channel. 


\subsubsection{Flow Development along the Upper Wall of the Case with Wall-Normal}

\section{Gravitational Acceleration}

The streamwise distribution of viscous length scale on the upper wall of the case with wall-normal gravitational acceleration is shown in Figure 5-30, along with the fixedproperty case and the non-buoyant, variable-property case. Wall-normal acceleration is observed to notably increase the viscous length scales along the upper channel wall. As will be shown, the prevailing buoyancy force reduces wall-normal turbulent mixing, yielding a less full velocity profile of the mean streamwise velocity, resulting in lower wall-shear, and hence higher viscous length scales. Figure 5-31 illustrates the distribution of velocity streaks along the upper wall of the case with wall-normal gravitational acceleration. Since the magnitude of $\delta$ is the same for all of the test cases, the $\delta$ normalized length scales follow the same trends as their respective absolute dimensions. Thus, the flood-plots in Figure 5-31(a) indicate that the near-wall streaks at $x_{\delta}=1.5$ are spaced farther apart in absolute dimensions $\left(\Delta z_{\delta}=0.51 \pm 0.04\right)$ than in the upstream

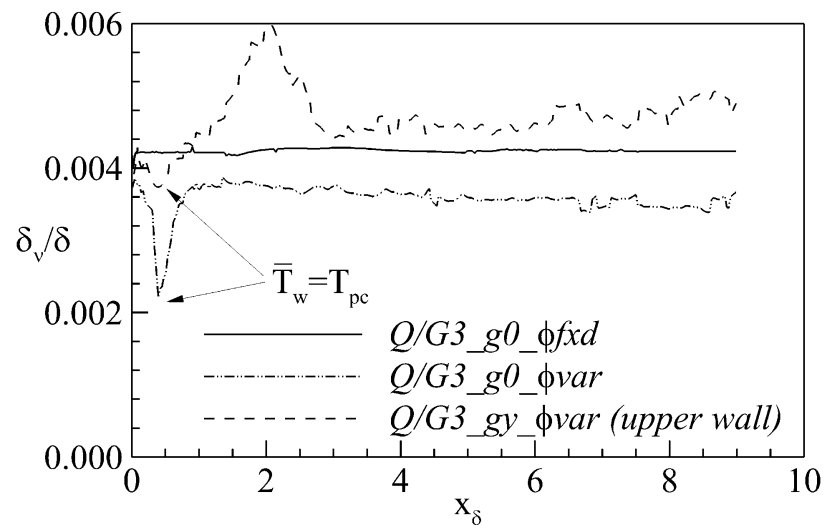

Figure 5-30: Streamwise distribution of the normalized viscous length scale for the heated-wall, fixed-property case, the non-buoyant, variable-property case, and the upper wall of the case with wall-normal gravitational acceleration. 


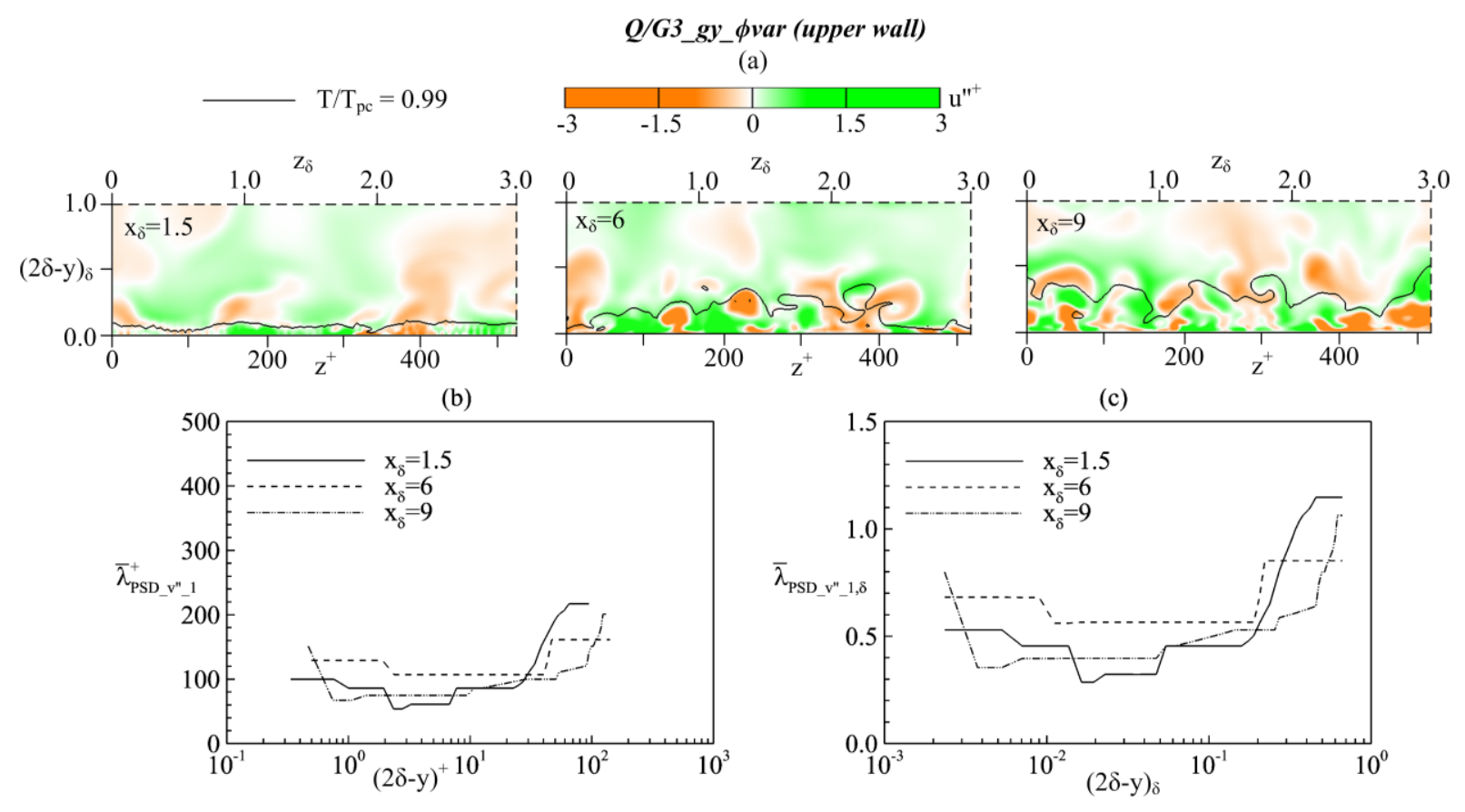

Figure 5-31: Velocity streaks on the upper wall of the channel in the presence of wallnormal gravitational acceleration identified by: a) flood-plots of the instantaneous streamwise component of perturbation velocity, $u^{\prime \prime}$; b-c) wall-normal distribution of $\bar{\lambda}_{P S D_{-} v_{-} 1}$, normalized by $\delta_{v}(\mathrm{~b})$ and $\delta(\mathrm{c})$.

domain $\left(\Delta z_{\delta}=0.44 \pm 0.02\right)$. However, the streaks in both cases have a mean spanwise spacing of $\Delta z^{+} \approx 100$. To illustrate the mechanism by which the near-wall streaks along the upper wall under the influence of wall-normal gravitational acceleration maintain a mean spanwise spacing of $\Delta z^{+} \approx 100 \pm 7$ while increasing in absolute spanwise spacing, the streaks are visualized in Figure 5-32 through flood-plots of the streamwise component of perturbation velocity in the streamwise/spanwise plane. As the streaks propagate from the unheated upstream domain onto the heated wall, the streaks most closely spaced together in the spanwise direction are observed to merge. For example, at a selected instance in time, $t_{\delta}=t_{0}$, the low-speed streaks labelled A and B in Figure 5-32 begin to 


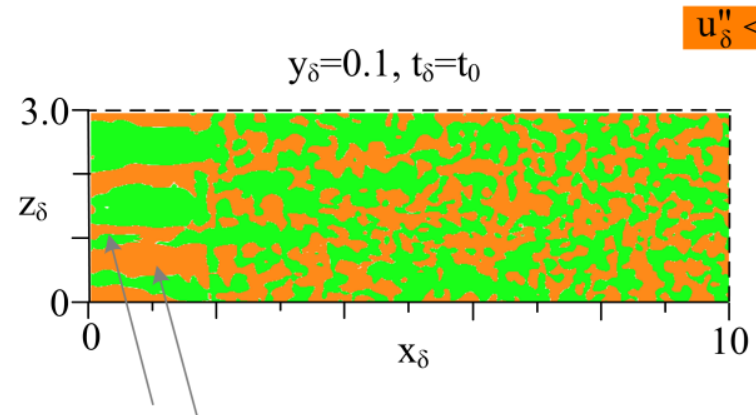

A

\section{$<u_{\delta}^{\prime \prime}>0$}

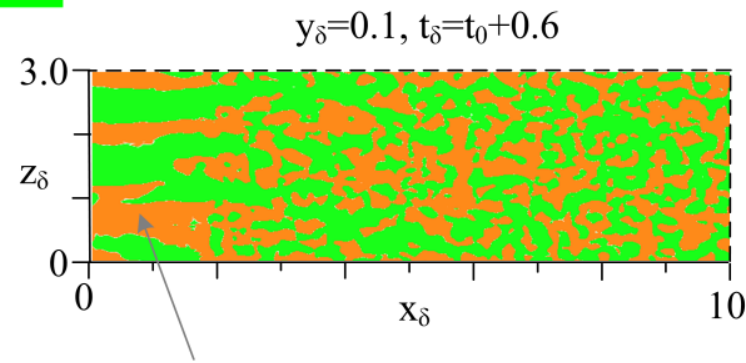

$\mathrm{AB}$

Figure 5-32: Distribution of velocity streaks along the upper wall of the case with wallnormal gravitational acceleration visualized through flood-plots of the streamwise component of perturbation velocity.

merge together to form a single low-speed streak labelled $\mathrm{AB}$ at $t_{\delta}=t_{0}+0.6$. Streaks at this stage of development and their corresponding wavepackets would not yet undergo spanwise merging in the fixed-property case. In Figure 5-32, downstream of $x_{\delta}=2.0$ the near-wall streaks are observed to quickly breakdown into smaller scales for the same reasons discussed for the non-buoyant, variable-property case in Section 5.4.2.2. The topology of streaks that form around near-wall hairpin vortices along the upper wall is illustrated in Figure 5-33 from observations of over 10 instances of such vortices. The gravitational acceleration vector is oriented in the $-\mathrm{y}$ direction, thus high-temperature streaks created by ejection motions have a buoyancy force acting on these streaks in the +y direction. The wall-normal acceleration due to the prevailing buoyancy force occurs in the vicinity of spanwise density gradients near the legs of the hairpin vortices to produce streamwise vorticity via the baroclinic mechanism, indicated in Figure 5-33. This vorticity has a sense of rotation opposite to the streamwise component of vorticity in the legs of the hairpin vortices, thus opposing the ejection and sweep motions on the inboard 


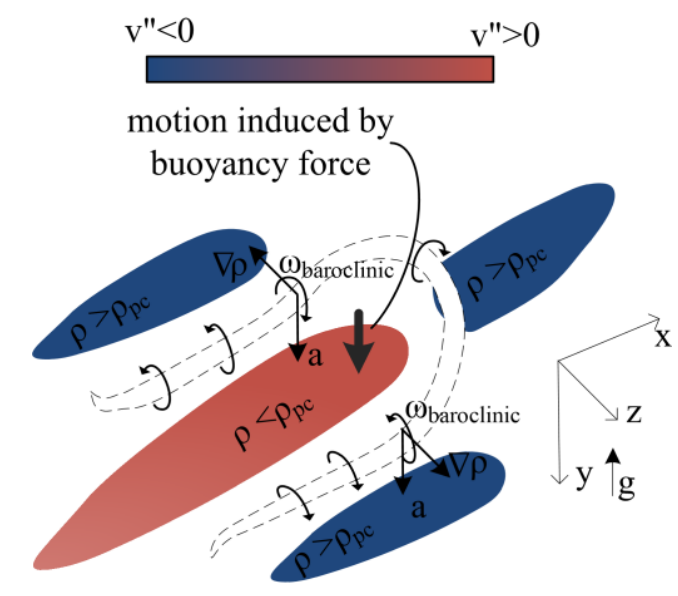

Figure 5-33: Illustration of the generation of vorticity through the baroclinic instability mechanism along the upper wall of the case with wall-normal oriented gravitational acceleration.

and outboard sides of the hairpin vortices. Opposition to these motions reduces their intensity in comparison to those in the non-buoyant, variable-property case. It was noted in Section 5.1 that the streamwise-elongated near- wall streaks are induced by the ejection and sweep motions in the vicinity of wavepackets of hairpin vortices, and that the shear layer surrounding these streaks is inviscid unstable, leading to grouping of vorticity to form subsequent hairpin vortices. It was also noted in Section 5.4.2.3 that regeneration of turbulence occurred when sweep motions on the outboard sides of a hairpin vortex induced secondary hairpin vortices on the outboard sides of the primary hairpin vortex. Accordingly, the generation of baroclinic vorticity along the upper wall of the case with wall-normal gravitational acceleration suppresses the mechanisms by which the near-wall hairpin vortices generate streaks and secondary hairpin vortices, and hence reduces the overall turbulent mixing. 


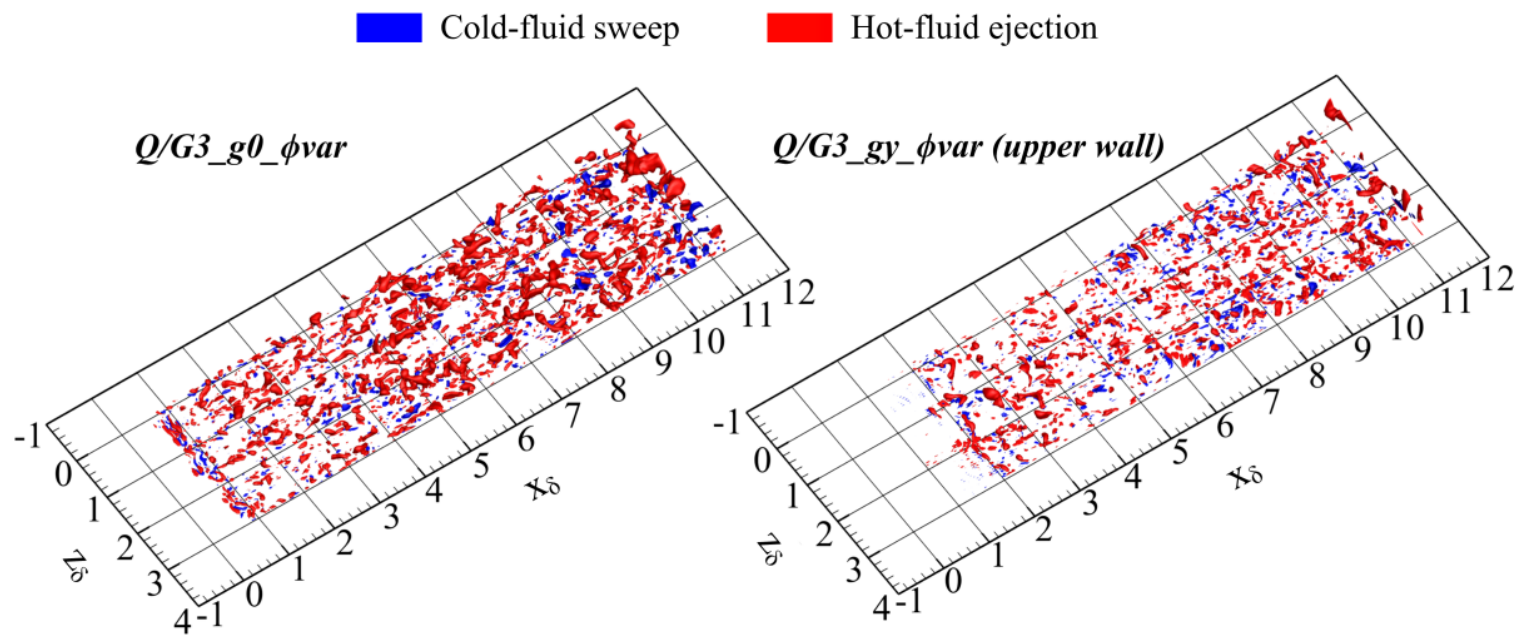

Figure 5-34: Iso-surfaces of $\left(v^{\prime \prime} T\right)^{+}=1 \times 10^{-4}$ for: a) the non-buoyant, variable-property case; b) the upper wall of the case with wall-normal gravitational acceleration.
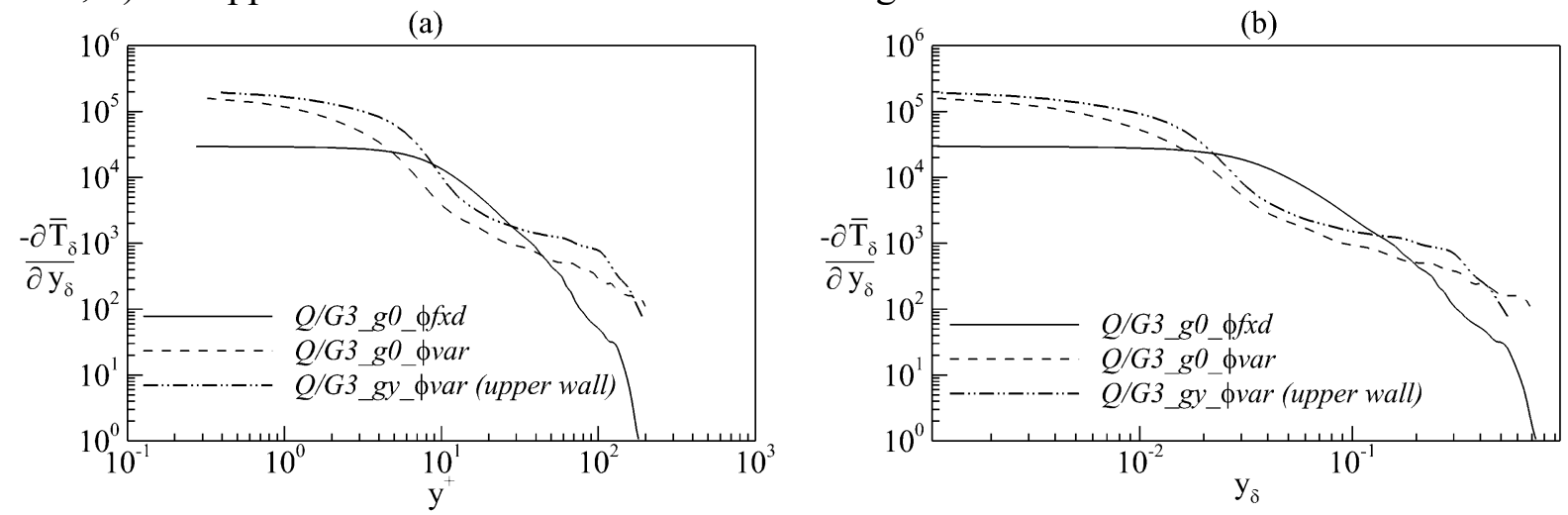

Figure 5-35: Wall-normal profile of the wall-normal temperature gradient for the nonbuoyant cases, and the upper wall of the case with wall-normal gravitational acceleration at $x_{\delta}=9$ plotted against: a) $y^{+}$; b) $y_{\delta}$.

The reduction in turbulent mixing due to the buoyancy force along the upper wall expectedly also yields reduced degrees of thermal mixing as visualized through isosurfaces of $\left(v^{\prime \prime} T^{\prime}\right)^{+}=1 \times 10^{-4}$, shown in Figure 5-34. These iso-surfaces reveal smaller regions of hot-fluid ejection and cold-fluid sweep motions when compared to the nonbuoyant, variable-property case. Likewise, the wall-normal profile of the wall-normal temperature gradient at $x_{\delta}=9$, shown in Figure 5-35, depicts overall higher wall-normal 
temperature gradients than those in the non-buoyant, variable-property case. Therefore, the net effect of buoyancy-driven instabilities, under the present flow conditions and wall-normal acceleration, is to deteriorate the heat transfer mechanisms along the upperwall of the channel.

\subsubsection{Flow Development in the Case with Downstream-Oriented Gravitational}

\section{Acceleration}

Figure 5-36 shows the streamwise distribution of viscous length scales for the heatedwall, fixed-property case, the lower wall of the case with wall-normal gravitational acceleration, and the case with downstream-oriented gravitational acceleration. The distribution of viscous length scales for the latter two cases are similar, suggesting similarity in the spatial scales of the coherent structures of turbulence in these two cases. Indeed, in Figure 5-37(a) the streaks that reside within $2 \delta$ streamwise distance of the onset of wall heating are observed to have a similar spatial distribution to those depicted in Figure 5-23(a) for the lower wall of the case with wall-normal gravitational acceleration. However, the differences in the profiles of $\bar{\lambda}_{P S D_{-} v_{-} 1}$ between Figure 5-37(b,c) and Figure 5-23(b,c) suggest some variations in the development of the hairpin vortices and associated streaks of these two cases. The topology of the streaks that form around a hairpin vortex is constructed in Figure 5-38 through observation of over 20 instances of such vortices in the case with downstream-oriented gravitational acceleration. The gravitational acceleration vector is oriented in the $+x$ direction, thus, lower-density streaks will have a net buoyancy force acting on them in the -x direction. 


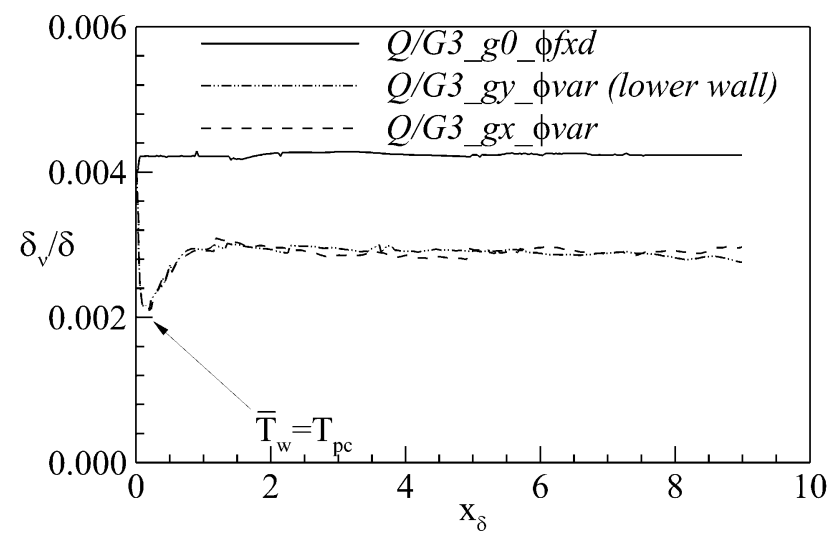

Figure 5-36: Streamwise distribution of the normalized viscous length scale for the heated-wall, fixed-property case, the lower wall of the case with wall-normal gravitational acceleration, and the case with downstream-oriented gravitational acceleration.

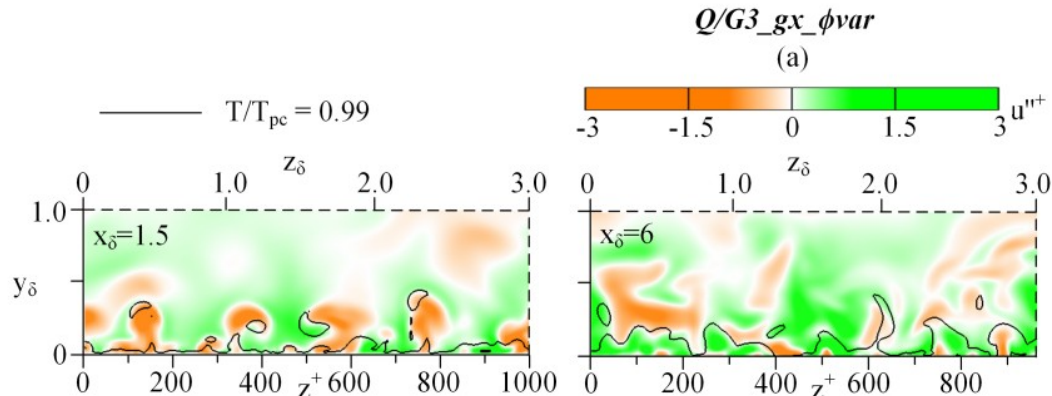

(b)

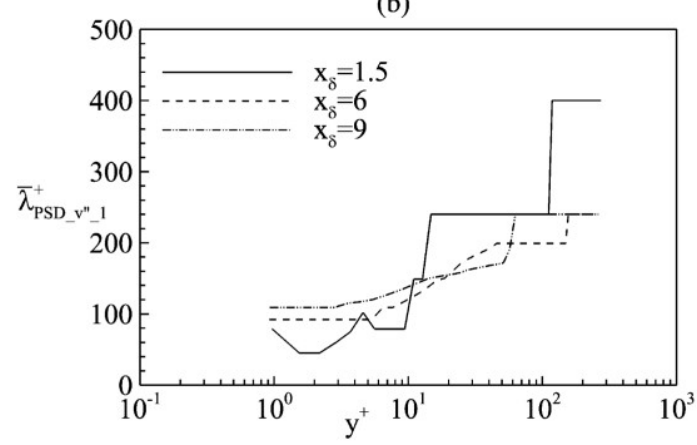

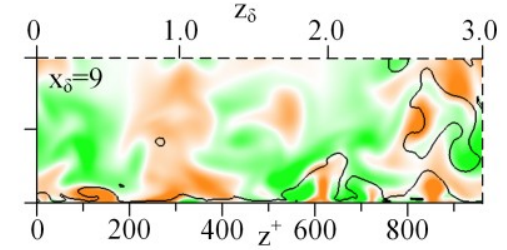

(c)

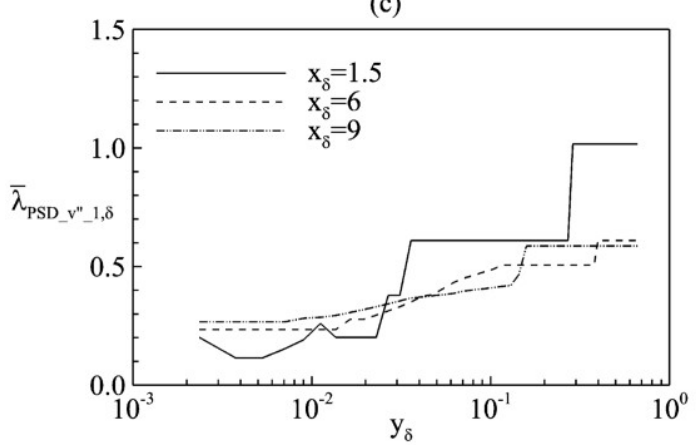

Figure 5-37: Velocity streaks in the case with downstream-oriented gravitational acceleration identified by: a) flood-plots of the instantaneous streamwise perturbation velocity component, $u^{\prime{ }^{+}}$; b-c) wall-normal distribution of $\bar{\lambda}_{P S D_{-} v_{-1} 1}$, normalized by $\delta_{v}(\mathrm{~b})$ and $\delta(\mathrm{c})$. 
Accordingly, as illustrated in Figure 5-38, the lower-density fluid between the legs of the hairpin vortex is decelerated in the streamwise direction. This deceleration and the spanwise density gradients near the legs of the hairpin vortex produce additional wallnormal vorticity via the baroclinic mechanism (Figure 5-13(b)) which is of the same sense of rotation as the prevailing wall-normal vorticity of these legs. The wall-normal vorticity is reoriented by the local mean strain rate such that it develops a streamwise component. Thus, the wall-normal and streamwise components of vorticity in the legs of the hairpin vortices are more intense in the case with downstream-oriented gravitational acceleration compared to those of the non-buoyant, variable-property case. The increased intensity of wall-normal vorticity component in the legs of the hairpin vortex decelerates the fluid residing underneath the head of the vortex in the streamwise direction, schematically depicted in Figure 5-38(b). This streamwise deceleration and the wallnormal density gradient near the head of the hairpin vortex produce spanwise vorticity again, via the baroclinic mechanism, with the same sense of rotation as the vorticity prevailing in the head of the hairpin vortex. The resultant more intense spanwise vorticity intensifies the sweep motion downstream of the hairpin vortex. This sweep motion transports colder fluid towards the wall. The budgets of streamwise vorticity (not shown), as per Equation 5-10, have a similar distributions and magnitudes to those shown in Figure 5-26 for the lower wall of the case with wall-normal gravitational acceleration. Several instances of the enhanced sweep motions are visible as blue-coloured iso- 


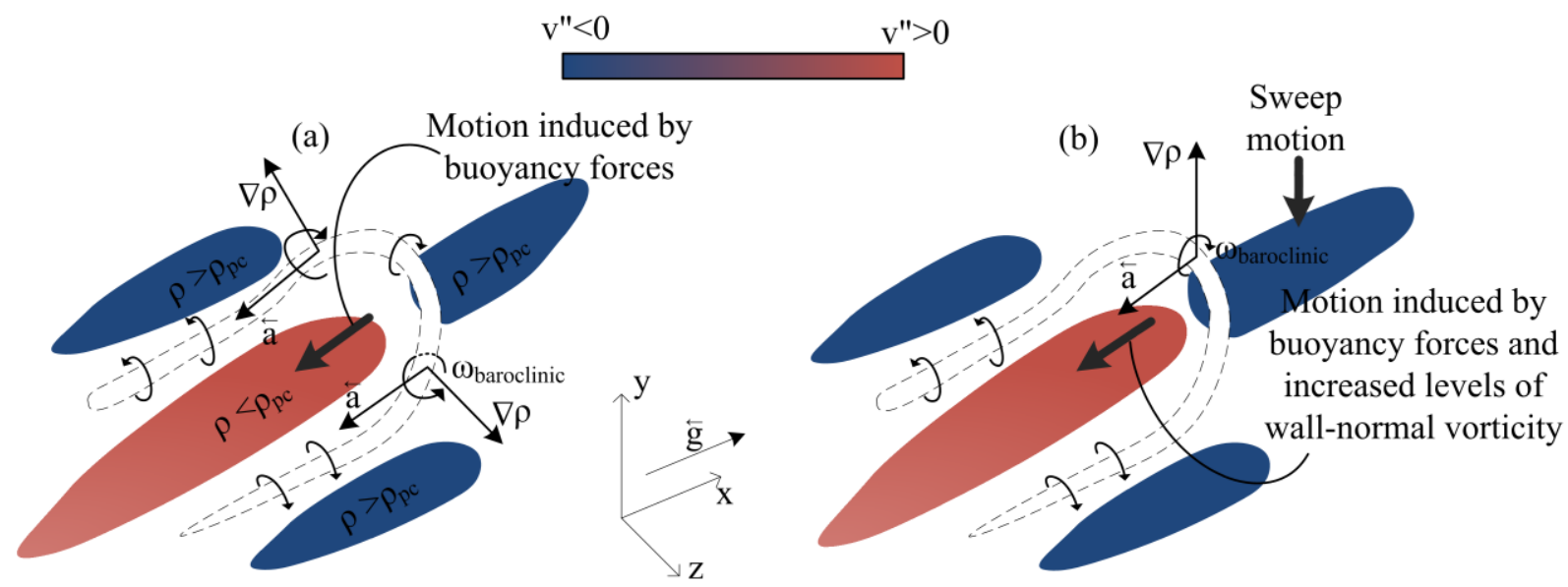

Figure 5-38: Illustration of the generation of vorticity through the baroclinic mechanism in a channel with downstream-oriented gravitational acceleration.

$\square$ Cold-fluid sweep $\square$ Hot-fluid ejection

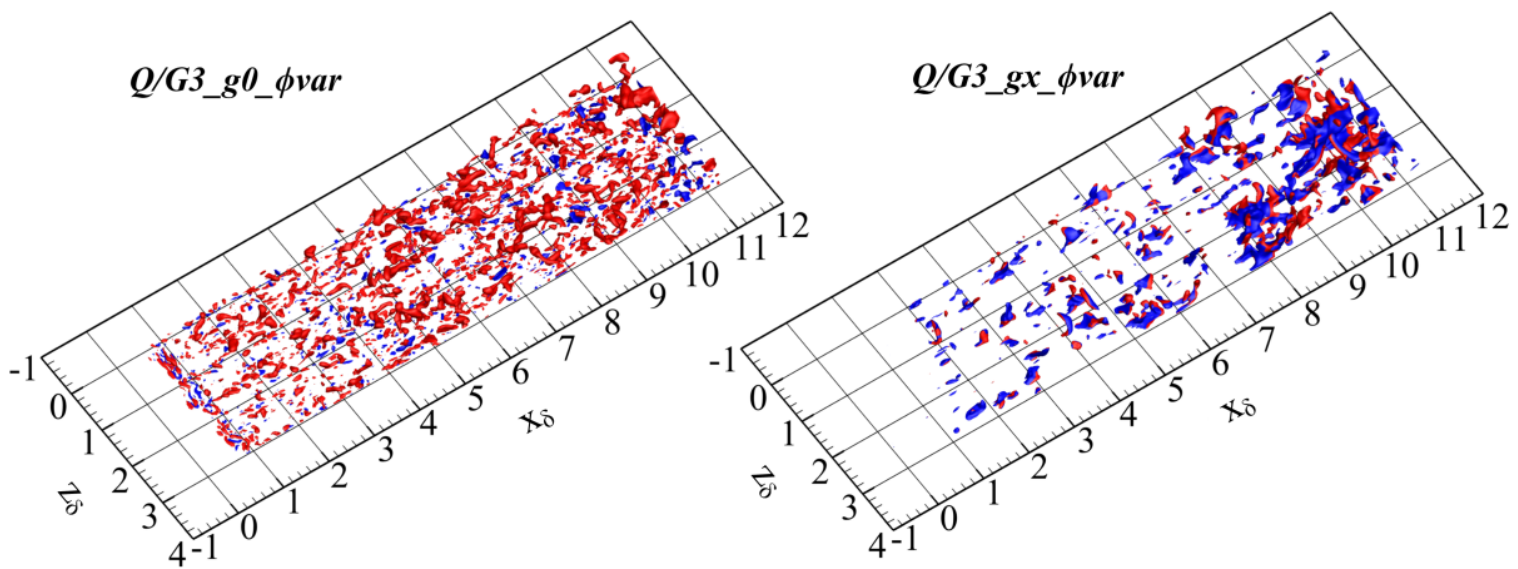

Figure 5-39: Iso-surfaces of $\left(v^{\prime \prime} T\right)^{+}=1 \times 10^{-4}$ for: a) the non-buoyant, variable-property case; b) the case with downstream-oriented gravitational acceleration.

surfaces in Figure 5-39, which shows iso-surfaces of $\left(v^{\prime \prime} T\right)^{+}=1 \times 10^{-4}$ for the non-buoyant, variable-property case, and the case with downstream-oriented gravitational acceleration. Notably, the wall-variable-based normalization of these iso- surfaces highlights the structures that most contribute to wall-normal thermal mixing in each case. It is observed 
for the latter case in this figure that larger-scale cold-fluid sweeps and hot-fluid ejections, occurring in close vicinity of one another, most significantly contribute to thermal mixing of the flow. These motions are the aforementioned sweep and ejection motions augmented by baroclinic vorticity generation respectively occurring downstream of the head and between the legs of the near-wall hairpin vortices, as illustrated in Figure 5-38. Since these motions enhance thermal mixing, the wall-normal profile of the wall-normal temperature gradient, shown in Figure 5-40 expectedly indicates lower temperature gradients in comparison to the non-buoyant, variable-property case. Thus, the net effect of buoyancy-driven instabilities, under the present flow conditions, is an enhancement of heat transfer mechanisms in the case with downstream-oriented gravitational acceleration.
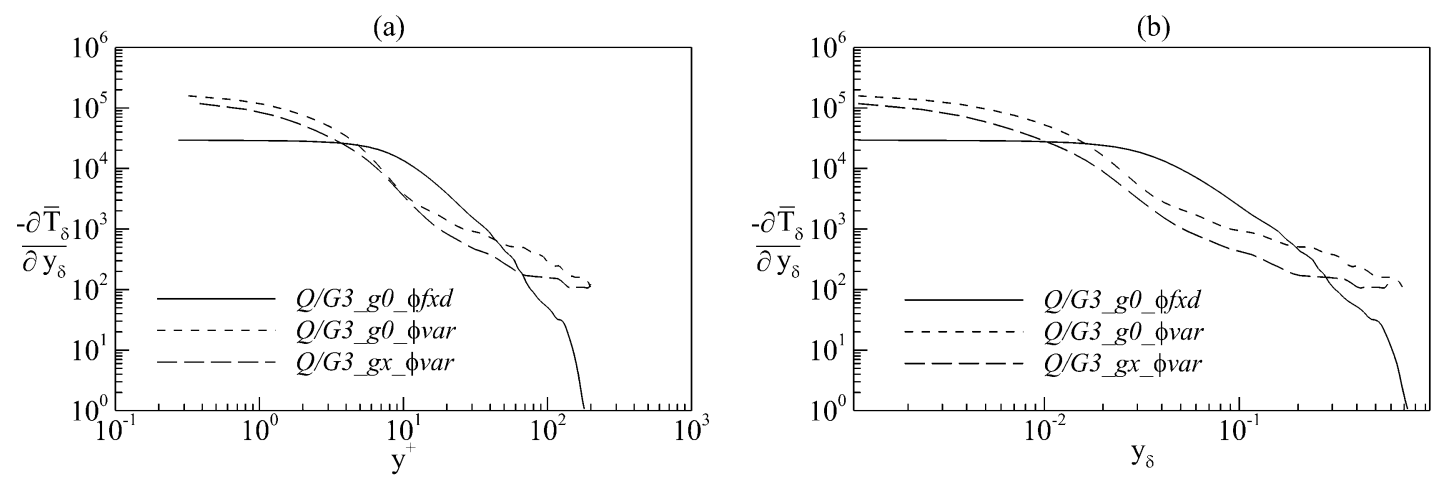

Figure 5-40: Wall-normal profile of the wall-normal temperature gradient for the nonbuoyant cases, and the case with downstream-oriented gravitational acceleration at $x_{\delta}=9$ plotted against: a) $y^{+}$; b) $y_{\delta}$. 


\section{Chapter: Similarity Criteria for Modelling Mixed-Convection Heat transfer in Ducted Flows of Supercritical Fluids}

In the previous two chapters it was demonstrated that spatial gradients of density and dynamic viscosity for flows of supercritical working fluids affect the coherent structures of turbulence, thus affecting thermal mixing of the flow in comparison to flows of subcritical single-phase fluids. In this chapter, a set of dynamic and state-space similarity criteria is developed for ducted flows of supercritical fluids. These criteria are validated with experimental data from published literature.

Citation: Azih, C. \& Yaras, M.I., 2017, 'Similarity criteria for modelling mixedconvection heat transfer in ducted flows of supercritical fluids', Journal of Heat Transfer, 139(12), p.122501.

\subsection{Introduction}

A key subject of interest for technologies that use fluids at the supercritical thermodynamic state is the development of prediction methods that capture the thermalhydraulics at this state (De Rosa et al., 2011; Pioro \& Duffy, 2005). In particular, the heat transfer behaviour of such fluids is of interest near the pseudo-critical state, which is a state where the specific heat value is maximized for a given pressure. In the vicinity of the pseudo-critical state, the thermophysical properties of the fluid change significantly with small changes in temperature. It is near this state that the characteristics of heat transfer in supercritical fluids are observed to differ from those of subcritical single-phase 
fluids. Development of correlations to reliably predict rates of convection heat transfer requires knowledge of the full set of the relevant similarity criteria. Knowledge of these criteria is also required to relate the convection heat transfer rates measured in setups with dissimilar working fluids. Identification of the similarity criteria in the latter context is often referred to as fluid-to-fluid modelling (Ambrosini, 2011; Pioro ${ }^{b}$ et al., 2004).

\subsubsection{Dynamic Similarity Criteria for Heat Transfer in Supercritical Fluids}

Heat transfer involving working fluids in the supercritical state features the well known phenomena of deteriorated heat transfer (DHT) and improved heat transfer (IHT), whereby the values of the convection heat transfer coefficient are respectively lower and higher by a notable margin than values predicted for subcritical single-phase fluids at equivalent flow conditions. The present understanding of the thermalhydraulics that lead to DHT and IHT is largely based on direct numerical simulations performed using simple geometries. These studies have established that DHT and IHT phenomena originate primarily from changes in the structure of turbulence under the action of inertial and/or buoyancy forces that stem from spatial gradients in the thermophysical properties of the fluid (Reinink \& Yaras, 2015; Dave et al., 2013; Bae et al., 2008). Although correlations calibrated using subcritical single-phase fluids may indicate a forced-convection heat transfer mode for flows exhibiting DHT and IHT phenomena, the mixed-convection heat transfer mode better represents such flows; as such flows may feature both inertial and buoyancy-driven phenomena (Jackson et al. 2011; Metaias \& Eckert, 1964). 
Non-dimensional forms of the mass, momentum, and energy conservation equations that are applicable to the mixed-convection heat transfer mode are given in Equations 6-1 to 6-3, respectively. These equations are derived in Appendices C.1 to C.4.

$$
\begin{gathered}
\frac{\partial \rho^{*}}{\partial t^{*}}+\frac{\partial}{\partial x_{j}^{*}}\left(\rho^{*} u_{j}^{*}\right)=0 \\
\frac{\partial}{\partial t^{*}}\left(\rho^{*} u_{j}^{*}\right)+\frac{\partial}{\partial x_{j}^{*}}\left(\rho^{*} u_{j}^{*} u_{i}^{*}\right)=-\frac{\partial p^{*}}{\partial x_{j}^{*}}+\frac{\partial \tau_{i j}^{*}}{\partial x_{j}^{*}}+\rho^{*} R i_{i} \\
\frac{\partial}{\partial t^{*}}\left(\rho^{*} h^{*}\right)+\frac{\partial}{\partial x_{j}^{*}}\left(\rho^{*} u_{j}^{*} h^{*}\right)=\frac{\partial}{\partial x_{j}^{*}}\left(\frac{1}{\left.\operatorname{RePr} \frac{k^{*}}{C_{p}^{*}} \frac{\partial h^{*}}{\partial x_{j}^{*}}\right)}\right.
\end{gathered}
$$

where:

$$
\begin{gathered}
\tau_{i j}^{*}=\frac{\mu^{*}}{R e}\left(\frac{\partial u_{i}^{*}}{\partial x_{j}^{*}}+\frac{\partial u_{j}^{*}}{\partial x_{i}^{*}}-\frac{2}{3} \frac{\partial u_{n}}{\partial x_{n}} \delta_{i j}\right) \\
R e=\left(\rho_{r e f} u_{r e f} L_{r e f}\right) / \mu_{r e f} \\
R i=\left(L_{r e f}\right) / u_{r e f}^{2} \\
\operatorname{Pr}=\left(\mu_{r e f} C_{p, r e f}\right) / k_{r e f}
\end{gathered}
$$

In these equations, $\tau_{i j}$ is the $i j$ component of the viscous stress tensor; $h$ is the static enthalpy. The * superscript indicates a non-dimensional form of a parameter, with the reference length $\left(L_{r e f}\right)$ and velocity $\left(u_{r e f}\right)$ scales and reference thermodynamic properties $\left(\rho_{r e f}, \mu_{\mathrm{ref}}, k_{r e f}\right.$, and $\left.C_{p, r e f}\right)$ used for this non-dimensionalization. Two main assumptions are employed in the formulation of Equation 6-3, namely that heat transfer occurs through a constant-pressure process and that the contribution of viscous dissipation to the energy balance is negligible. Based on Equation 6-1 to 6-7, it can be surmised that in heated flows which feature significant inertia- and buoyancy-driven phenomena, for a specific state-space distribution of thermodynamic state variables $\rho^{*}, \mu^{*}, C_{p}{ }^{*}, k^{*}, h^{*}$, and $p^{*}$, the 
Reynolds (Re), Richardson (Ri), and Prandtl (Pr) numbers should be sufficient to establish dynamic similarity.

Dynamic similarity criteria for mixed-convection heat transfer in supercritical fluids were proposed by Ambrosini (2011), Cheng et al. (2011) which were subsequently updated by Zahlan et al. (2014) and Jackson and co-workers (Jackson, 2011; McEligot \& Jackson, 2004; Jackson \& Hall,1979). Some of the parameters that make up these similarity criteria are common with those proposed for hydrodynamic instability of such flows, with the pioneering work of Zuber (1966), the works of Chatoorgoon $(2013,2008)$ and those of Ambrosini $(2011,2008)$ being several examples. Ambrosini (2011) proposed $R e, R i$, and the Peclet number, $P e=R e P r$, as the dynamic similarity criteria, noting that $P e$ appears in the non-dimensional energy conservation equation. Cheng et al. (2011) proposed $R e, R i$, and $P r$ as the set of dynamic similarity criteria. In particular, Cheng et al. [11] proposed $\operatorname{Re} P r^{n}$ as a scaling parameter, where $n=0.42$ is based on coefficients of the Dittus-Boelter equation (Winterton, 1998). Zahlan et al. (2014) recognized that empirical Nusselt number correlations developed for heat transfer in flows of supercritical fluids have optimal coefficients that differ from the Dittus-Boelter values. Accordingly, these authors proposed an alternate value of $n=0.63$. Both Ambrosini (2011) and Cheng et al. (2011) proposed an additional similarity criterion in the form of a non-dimensional wall heat transfer rate, $Q^{*}$, obtained from the heat transfer boundary condition at the wall. This boundary condition equates the heat flux from the wall to the conductive heat flow through the fluid at the wall. If the enthalpy is non-dimensionalized by a reference enthalpy given as $h_{r e f}=C_{p}$, ref $/ \beta_{r e f}$, then: 


$$
-\frac{1}{C_{p, w}^{*}}\left(\frac{\partial h^{*}}{\partial y^{*}}\right)_{w}=\underbrace{\frac{\dot{Q}_{w}^{\prime \prime} L_{r e f} \beta_{r e f}}{k_{w}}}_{Q^{*}}
$$

where, $y$ is the wall-normal Cartesian spatial coordinate, the " superscript indicates that the property value is evaluated per unit area, and the $\cdot$ accent indicates time rate. Alternative forms of $Q^{*}$ can be defined; for example, with a reference enthalpy defined as $h_{r e f}=C_{p, r e f} T_{r e f}:$

$$
-\frac{1}{C_{p, w}^{*}}\left(\frac{\partial h^{*}}{\partial y^{*}}\right)_{w}=\underbrace{\frac{\dot{Q}_{w}^{\prime \prime} L_{r e f}}{k_{w} T_{r e f}}}_{Q^{*}}
$$

where $T$ is the temperature. For ideal gases, Equations 6-8 and 6-9 are equivalent since $\beta_{\text {ref }}=1 / T_{\text {ref; }}$, however, $\beta_{\text {ref }}$ and $1 / T_{\text {ref }}$ may differ by up to three orders of magnitude for supercritical fluids. Several expressions that quantify the non-dimensional wall heat transfer rate in supercritical fluids have been proposed in the published literature and are tabulated in Table 6-1. In the table, $m$ is the fluid mass and $d_{h}$ is the hydraulic diameter of the flow path. The expression of $Q^{*}$ proposed by McEligot $\&$ Jackson (2004) is obtained by dividing Equation 6-8 by $P e$. Ambrosini's (2011) expression of $Q^{*}$ is obtained by multiplying Equation 6-8 with the ratio of the wall-heat-transfer surface area to the flow area and dividing by $P e$. The $Q^{*}$ expression by Cheng et al. (2011) is obtained from Equation 6-9 with $T_{r e f}=T_{p c}-T_{c}$, and that of Jackson \& Hall (1979) is obtained from Equation 6-9 with $T_{r e f}=T_{b}$. The version of $Q^{*}$ presented by Jackson (2011) is in the form of Equation 6-8. In addition to the respective $Q^{*}$ values tabulated in Table 6-1, Jackson and co-workers (Jackson, 2011; McEligot \& Jackson, 2004; Jackson \& Hall, 1979) suggested $\operatorname{Re}, \operatorname{Pr}$, and $G r$ as the dynamic similarity parameters for mixed-convection heat 
Table 6-1: Non-dimensional expressions for wall heat transfer.

\begin{tabular}{lc}
\hline Author & Dimensionless wall heat transfer rate $\left(Q^{*}\right)$ \\
\hline Ambrosini (2011) & $\frac{\dot{Q}_{w} \beta_{p c}}{\dot{m} C_{p, p c}}$ \\
Cheng et al. (2011) & $\frac{\dot{Q}_{w}^{\prime \prime} d_{h}}{k_{b}\left(T_{p c}-T_{c}\right)}$ \\
Jackson (2011) & $\frac{\dot{Q}_{w}^{\prime \prime} \beta_{b} d_{h}}{k_{b}}$ \\
Jackson \& Hall (1979) & $\frac{\dot{Q}_{w}^{\prime \prime} d_{h}}{k_{b} T_{b}}$ \\
McEligot \& Jackson (2004) & $\frac{\dot{Q}_{w}^{\prime \prime} \beta_{b}}{G C_{p, b}}$ \\
\hline
\end{tabular}

transfer in supercritical fluids. $G r$ is the Grashof number defined as:

$$
G r=\left(g \beta_{r e f} \Delta T_{r e f} L_{r e f}^{3}\right) / v_{r e f}^{2}
$$

where, $\Delta T_{r e f}=T-T_{\text {ref. }} G r$ is one of the relevant similarity criteria for free-convection flows, driven primarily by buoyancy forces. A modified Richardson number is conventionally derived based on the Grashof number:

$$
R i^{\prime}=G r / R e^{2}=R i \beta_{r e f} \Delta T_{r e f}
$$

\subsubsection{State-Space Similarity Criteria for Supercritical Fluids}

To establish similarity in state space, $p^{*}=p / p_{\mathrm{c}}$ is generally selected as one of two independent state-space parameters. For the second independent state-space parameter, Jackson and Hall (1979) proposed $T^{*}=T / T_{c}$ and Bogachev et al. (1984) proposed $T / T_{p c}$ as the non-dimensional temperature. Cheng et al. (2011) demonstrated non- 
dimensionalization using characteristic values at the pseudo-critical state rather than the critical state to be more suitable for establishing similarity in state space through comparison of the state-space distributions of $\rho, \mu, C_{p}$, and $k$ for water, carbon dioxide $\left(\mathrm{CO}_{2}\right)$, and refrigerant $\mathrm{R} 134 \mathrm{a}$. These authors also proposed a temperature ratio, defined as $\left(T-T_{p c}\right) /\left(T_{p c}-T_{c}\right)$, as an alternative to $T / T_{p c}$ or $T / T_{c}$. However, this temperature ratio is singular at the critical state and sensitive to small uncertainties in the state variables near the critical state Zahlan et al. (2014). Based on literature on boiling heat transfer, Ambrosini (2011) proposed a non-dimensional enthalpy, defined as $h^{*}=\left(h-h_{p c}\right)\left(\beta_{p c} / C_{p, p c}\right)$, in place of a non-dimensional temperature, arguing that enthalpy is the relevant independent criterion for flows where the variation in $C_{p}$ with $T$ is not negligible.

\subsubsection{Validation of Similarity Criteria for Heat Transfer in Supercritical Fluids}

Dynamic similarity criteria and state-space similarity criteria may be used to develop fluid-to-fluid models. Validation of these models for mixed-convection heat transfer in supercritical fluids is hampered due to lack of datasets having values of the similarity criteria that are consistent for multiple working fluids. To circumvent this difficulty, Jackson (2011) and McEligot \& Jackson (2004) established criteria for the onset of significant buoyancy- and inertia-induced heat-transfer phenomena for ducted flows of supercritical fluids. These criteria were established through semi-empirical methods with

data from experiments that used air at atmospheric pressure. Jackson (2011) proposed that buoyancy forces become significant when:

$$
\frac{G r_{b}}{R e_{b}^{3.425} \operatorname{Pr}_{b}^{0.8}}>2 \times 10^{-7}
$$


and acceleration effects due to volumetric expansion of the near-wall fluid become significant when:

$$
\frac{Q^{*}}{R e_{b}^{1.625} \operatorname{Pr}_{b}}>2 \times 10^{-6}
$$

where, $Q^{*}$ is the non-dimensional wall heat transfer rate proposed by Jackson (2011) as listed in Table 6-1. Jackson (2011) proposed that the characteristic properties in the formulations of $R e, G r$, and $\operatorname{Pr}$ be evaluated at bulk-fluid conditions as denoted by the subscript $b$ in Equations 6-12 and 6-13. For the criteria expressed by Equations 6-12 and 6-13 Jackson (2011) showed that the measured heat transfer coefficients significantly differ from those predicted by correlations calibrated for subcritical single-phase fluids. The data used for this comparison consisted of sets of supercritical $\mathrm{CO}_{2}$ channel flow data obtained over an inlet Reynolds number range of 2,900 to 10,600 at a fixed nondimensional pressure of $p / p_{c}=1.16$. Cheng et al. (2011) used their similarity criteria consisting of $R e, R i, P r$, and $Q^{*}$, with characteristic values evaluated at the bulk flow conditions, to transform data obtained with $\mathrm{CO}_{2}$ working fluid to water-equivalent conditions. These authors then assessed how adequately the transformed data was modelled by the heat-transfer correlation of Bishop et al. (1964) developed for water at the supercritical state. Despite the moderate success achieved using this method, its applicability is limited by the well known limitations of Bishop et al.'s (1964) heattransfer correlation in predicting DHT and IHT trends (Pioro ${ }^{\mathrm{b}}$ et al. 2004). Ambrosini et al. $(2011,2008)$ assessed the validity of their proposed set of dynamic similarity criteria, namely $R e, R i, P e$, and $Q^{*}$, by numerically solving the RANS equations with a low-Re $k-\varepsilon$ turbulence closure for heated pipe flow. The reference velocity was taken as the inflow 
velocity, the reference density was selected as the pseudo-critical density, and all other reference values were evaluated at the bulk-fluid state. The fluids used in the computational analyses were $\mathrm{CO}_{2}$, refrigerant $\mathrm{R} 23$, ammonia $\left(\mathrm{NH}_{3}\right)$, and water. Based on these computations, Ambrosini (2011) noted significant discrepancies in the nondimensional heat transfer rates presented via the Nusselt number. The analysis was subsequently improved through more optimal values of the coefficients in the RANS based formulation as pertains to heat transfer in flows of supercritical fluids (Ambrosini, 2008). However, discrepancies are still expected in such formulations since the Boussinesq hypothesis is inherent in the formulation of zero-, one-, and two-equation turbulence closures. This hypothesis presumes the turbulent mixing to be locally isotropic and dominance of the production and dissipation mechanisms in the turbulence budget which are not supported by the observations of Bae et al. (2008), Azih et al. (2012) based on direct numerical simulations of heated duct flows of supercritical fluids.

The foregoing suggests that more research is needed on identifying the dynamic similarity criteria and state-space similarity criteria affecting mixed-convection heat transfer in supercritical fluids. The present study proposes a set of similarity criteria for mixed-convection heat transfer in supercritical fluids established through our current understanding of flow dynamics and convection heat transfer at this thermodynamic state. A fluid-to-fluid model is proposed based on these similarity criteria and validated using published experimental data. 


\subsection{Dynamic Similarity Parameters for Heat Transfer in Supercritical Fluids}

In the following paragraphs, the dynamic similarity criteria that are deemed to fully define the dynamics of mixed-convection heat transfer in supercritical fluids are discussed from a physical standpoint to assist in making judicious choices for evaluating the reference parameters that make up their mathematical expressions.

The Reynolds number represents the ratio of inertial to viscous forces. The inertial force per unit area may be defined as $G_{I} u_{I}$, and the viscous force per unit area can be expressed as $\mu(\partial u / \partial L)_{\mu}$. Accordingly:

$$
R e=\frac{G_{I} u_{I}}{\mu(d u / d L)_{\mu}}
$$

Letting

$$
(\partial u / \partial L)_{\mu} \approx(\Delta u / \Delta L)_{\mu}=u_{\mu} / L_{\mu}
$$

results in:

$$
R e \approx \frac{G_{I} L_{\mu}}{\mu} \frac{u_{I}}{u_{\mu}}
$$

where the subscripts $I$ and $\mu$ indicate reference parameters characteristic of inertial and viscous forces, respectively. For example, the flow length scale selected to quantify $L_{\mu}$ must be relevant to viscous effects in the flow. The velocity ratio, $u_{I} / u_{\mu}$, may be omitted from Equation 6-14(c) if $u_{I}$ is proportional to $u_{\mu}$ for the subject class of flows, yielding:

$$
R e \propto \frac{G_{I} L_{\mu}}{\mu}
$$

For example, the friction velocity in a fully developed pipe flow, $u_{\mu} \equiv u_{\tau}=\left(\tau_{w} / \rho_{w}\right)^{0.5}$, can be expressed in terms of the bulk-fluid mean axial velocity, $u_{I} \equiv u_{b}$, through the 
Colebrook correlation (Colebrook \& White, 1937). In heated flows of supercritical working fluids, local flow acceleration near the heated surface is driven primarily by the rapid decrease in density with increasing temperature near the pseudo-critical state of the working fluid (Reinink \& Yaras, 2015; Jackson, 2011). Thus, in applying Equation 6-14(c) to heat transfer in supercritical fluids, the net contribution of near-wall acceleration should be adequately accounted for.

The Richardson number represents the ratio of buoyancy to inertial forces. The buoyancy force per unit volume $\left(f_{B}\right)$ can be quantified by:

$$
f_{B}=\left(\rho_{B}-\rho\right) g
$$

where the subscript $B$ denotes a relevant reference parameter for the buoyancy force. From the definition of the volumetric expansion coefficient:

$$
\left.\beta_{B}=-\frac{1}{\rho_{B}}\left(\frac{\partial \rho}{\partial T}\right]_{\mathrm{p}}\right)_{\mathrm{B}} \approx-\frac{1}{\rho_{B}}\left(\frac{\Delta \rho}{\Delta T}\right)_{\mathrm{B}} \approx-\frac{1}{\rho_{B}} \frac{\rho-\rho_{B}}{T-T_{B}}
$$

Although this approximation is only valid for small changes in density with temperature change, it will be shown in the validation exercise in Section 6.5 that the relevant flow physics is captured despite this simplifying approximation. Hence, from Equations 6-15 and 6-16,

$$
f_{B}=g \rho_{B} \beta_{B}\left(\Delta T_{B}\right)
$$

where

$$
\Delta T_{B}=T-T_{B}
$$

The inertial force per unit volume may be expressed as $G_{I} u_{I} / L_{I}$. Thus, the Richardson number can be written as: 


$$
R i^{\prime} \equiv \frac{f_{B}}{f_{I}} \propto \frac{g \rho_{B} \beta_{B} \Delta T_{B}}{G_{I} u_{I} / L_{\mu}}=\frac{g L_{I}}{u_{I}} \beta_{B} \Delta T_{B} \frac{\rho_{B}}{G_{I}}
$$

Letting

$$
u_{I} \propto G_{I} / \rho_{I}
$$

results in:

$$
R i^{\prime} \propto \frac{g L_{I}}{G_{I}^{2} /\left(\rho_{B} \rho_{I}\right)} \beta_{B} \Delta T_{B}
$$

For flows heated at the wall, the temperature potential $\left(\Delta T_{B}\right)$ is conventionally taken as $T_{w}-T_{b}$; however, $T_{w}$ is typically only known if the detailed flow field is known. Fourier's law of heat conduction may be used to approximate $\Delta T_{B}$ :

$$
\dot{Q}_{w}^{\prime \prime}=-k_{w} \partial T / \partial L=k_{w} \Delta T_{k} / L_{k}
$$

The $k$ subscript indicates a reference parameter characteristic of conduction heat transfer, and these parameters are selected in a manner that is consistent with the definition of the Nusselt Number, $\mathrm{Nu}$, which will be used to validate the fluid-to-fluid model that is developed in Section 6.4 based on the similarity parameters developed in the present section. The Nusselt number represents the ratio of the wall-normal convection to wallnormal conduction heat transfer rates. The wall-normal convection heat transfer rate is expressed as:

$$
\dot{Q}_{w, \text { conv }}^{\prime \prime}=H\left(T_{w}-T_{b}\right)=H \Delta T_{B}
$$

where $H$ is the convection heat transfer coefficient. The wall-normal conduction heat transfer rate is expressed by Equation 6-19, therefore:

$$
N u=\frac{H \Delta T_{B}}{k_{w} \Delta T_{k} / L_{k}}=\frac{H L_{k}}{k_{w}} \frac{\Delta T_{B}}{\Delta T_{k}}
$$


To recover the conventional form of the Nusselt number, $\Delta T_{B}$ and $\Delta T_{k}$ need to be assumed to have comparable magnitudes. However, heat transfer experiments with supercritical fluids reveal significant changes in Nusselt number driven by changes in the wall heat flux, suggesting that the mechanisms of conduction and convection heat transfer may be highly uncorrelated such that $\Delta T_{B}$ and $\Delta T_{k}$ may be significantly dissimilar. Notwithstanding, it will be shown in the validation exercise in Section 6.5 that the relevant flow physics is captured despite this simplifying assumption. Noting Equation 6-19, with $\Delta T_{B}$ substituted for $\Delta T_{k}$, the Richardson number stated in Equation 6-18(c) can be rewritten as:

$$
R i^{\prime}=\frac{\rho_{B} \rho_{I} g L_{k} L_{I}}{G_{I}^{2}} \frac{\beta_{B} \dot{Q}_{w}^{\prime \prime}}{k_{w}}
$$

The Prandtl number, $P r$, represents the ratio of the momentum and thermal diffusion rates:

$$
\operatorname{Pr}=\frac{(v)}{(\alpha)}=\frac{\mu / \rho}{k /\left(\rho C_{p}\right)}=\frac{\mu}{k / C_{p}}
$$

where $v$ is the kinematic viscosity (i.e. momentum diffusivity) and $\alpha$ is the thermal diffusivity. Notably, $k$ and $C_{p}$ should both be evaluated at the same thermodynamic state for $\operatorname{Pr}$ to be most effective as a similarity criterion.

The $Q^{*}$ non-dimensional parameter captures the rate of heating of the fluid at the wall. Under steady-state, steady-flow conditions this rate of heating is equal to the heat transferred to the bulk fluid along the flow path. The rate of heating of the bulk fluid along the flow path is expressed as: 


$$
\dot{Q}=m\left(h_{o u t, b}-h_{i n, b}\right)
$$

Here the subscripts in and out respectively denote conditions at the beginning and end of the flow path being considered. Equation 6-24 shows that the rate of heating along the flow path per unit mass is equal to the change in bulk-fluid enthalpy. To define a nondimensional enthalpy, it is noted that inertial and buoyancy-driven phenomena observed in flows of supercritical fluids are particularly affected by spatial gradients of density (Reinink, \& Yaras, 2015; Jackson, 2011; Bae et al., 2008). Accordingly, an expression for the non-dimensional enthalpy, $h^{*}$, is derived in a manner that includes $\partial \rho / \partial h$ as follows:

$$
\left.h^{*}=-\frac{1}{\rho_{r e f}}\left(\frac{\partial \rho}{\partial h}\right]_{p}\right)_{r e f}\left(h-h_{r e f}\right)
$$

The expression of Equation 6-25(a) is formulated noting that enthalpy data of substances are published with respect to reference enthalpy values that are not consistent among all datasets or relevant to heat transfer in flows of supercritical fluids. Thus, the enthalpy value is quantified relative to a suitable reference enthalpy value, $h_{\text {ref. }}$ It follows from Equation 6-25(a) that:

$$
\begin{gathered}
h^{*}=\underbrace{\left.-\frac{1}{\rho_{r e f}}\left(\frac{\partial \rho}{\partial T}\right]_{p}\right)_{r e f}}_{\beta_{r e f}} \underbrace{\left.\left(\frac{\partial T}{\partial h}\right]_{p}\right)_{r e f}}_{\frac{1}{C_{p, r e f}}}\left(h-h_{r e f}\right) \\
h^{*}=\frac{\beta_{r e f}}{C_{p, r e f}}\left(h-h_{r e f}\right)
\end{gathered}
$$

Then, the non-dimensional streamwise change in the enthalpy of the fluid as a result of heating at the wall can be expressed as:

$$
h_{\text {out }}^{*}-h_{\text {in }}^{*}=\beta_{\text {ref }} / C_{p, r e f}\left(h_{\text {out }, b}-h_{i n, b}\right)
$$


Noting the thermal energy balance given in Equation 6-24, it follows that:

$$
h_{\text {out }}^{*}-h_{\text {in }}^{*}=\beta_{\text {ref }} / C_{p, r e f}(\dot{Q} / \dot{m})
$$

Without loss of generality, Equation 6-26(b) can be multiplied by a geometric similarity criterion, which requires similarity in the ratio of the flow area to heat-transfer area, yielding a non-dimensional wall heat transfer rate, $Q^{*}$ :

$$
Q^{*}=\beta_{r e f} / C_{p, r e f}\left(\dot{Q}_{w}^{\prime \prime} / G\right)
$$

\subsection{Similarity Criteria for the State-Space Distribution of Thermophysical Properties at the Supercritical State}

To develop similarity criteria that define the fluid in state space, the thermophysical properties that appear in the governing equations for fluid flow, given by Equations 6-1 to 6-3, are analyzed. These properties are: density $-\rho$; dynamic viscosity $\mu$; isobaric specific heat $-C_{p}$; and, the thermal conductivity $-k$. Non-dimensional pressure and enthalpy can be used as the two independent state variables to uniquely define the state of a pure fluid. The non-dimensional enthalpy is expressed as shown in Equation 6-25(c). To determine the reference values appearing in this equation, it is noted that the unique thermodynamic states relevant to fluids at supercritical pressures are the critical state and the pseudo-critical state. Through comparison of the state-space distributions of $\rho, \mu, C_{p}$, and $k$ for water, carbon dioxide $\left(\mathrm{CO}_{2}\right)$, and refrigerant R134a, Cheng et al. (2011) demonstrated that the pseudo-critical state better characterizes the thermodynamic state of the fluid than the critical state. They noted that nondimensionalization using the critical state as the reference state resulted in differences in 
both the trends and magnitude of the state-space distributions of $\rho, \mu, C_{p}$, and $k$ amongst the analyzed fluids, whereas choosing the pseudo-critical state as the reference state only yielded differences in magnitude of these properties. Accordingly, Equation 6-25(c) can be written as:

$$
h^{*}=\frac{\beta_{p c}}{C_{p, p c}}\left(h-h_{p c}\right)
$$

The properties $\rho, \mu, C_{p}$, and $k$ are similarly non-dimensionalized with reference values at the pseudo-critical state:

$$
\begin{aligned}
\rho^{*} & =\frac{\rho}{\rho_{p c}} \\
\mu^{*} & =\frac{\mu}{\mu_{p c}} \\
C_{p}^{*} & =\frac{C_{p}}{C_{p, p c}} \\
k^{*} & =\frac{k}{k_{p c}}
\end{aligned}
$$

Although the non-dimensional pressure and enthalpy are sufficient for defining the fluid in state space, for reference purposes, a non-dimensional temperature may be derived from the definition of $C_{p}{ }^{*}$ given as:

$$
\frac{C_{p}}{C_{p, p c}}=C_{p}^{*} \equiv \frac{\partial h^{*}}{\partial T^{*}}
$$

Then, using the definition of $h^{*}$ as stated in Equation 6-28:

$$
\begin{gathered}
\partial\left(T^{*}\right)=\frac{C_{p, p c}}{C_{p}} \partial\left(\frac{\beta_{p c}}{C_{p, p c}}\left(h-h_{p c}\right)\right)=\beta_{p c}\left(\frac{1}{C_{p}} \partial\left(h-h_{p c}\right)\right) \\
=\beta_{p c} \partial\left(T-h_{p c} / C_{p}\right)
\end{gathered}
$$

Integrating Equation 6-30(b) yields: 


$$
T^{*}=\beta_{p c}\left(T-h_{p c} / C_{p}\right)
$$

for which the integration constant has been set to a value of zero to ensure that $T^{*}=0$ corresponds to $h^{*}=0$. The dependencies of $\rho^{*}, \mu^{*}, k^{*}$, and $C_{p}{ }^{*}$ on the non-dimensional enthalpy, $h^{*}$, are illustrated for water, carbon dioxide, helium, and R134a in Figure 6-1 to Figure 6-4. The ordinates in these figures are shown in log-scale to facilitate an order of magnitude comparison of the analyzed fluids. The critical temperature and pressure for these fluids are listed in Table 6-2. The state-space distributions of $\rho^{*}$ shown in Figure 5-3 indicate favourable agreement between the fluids for several pressure ratios, $p / p_{c}$. In Figure 6-2, favourable agreement is also observed for the $\mu^{*}$ state-space distributions of R134a and carbon dioxide and likewise for the distributions for water and helium when $p / p_{c} \geq 1.13$. The trends for the variation of $k^{*}$ with $h^{*}$, shown in Figure 6-3, are similar for water, carbon dioxide, and R134a. For $p / p_{c} \leq 1.13, k^{*}$ is observed to decrease with $h^{*}$ until $h^{*}=-0.3$ above which an increase in $k^{*}$ prevails until the pseudocritical state, followed by a sharp decrease in $k^{*}$. Conversely, no significant variation of $k^{*}$ with $h^{*}$ is noted for helium in the $h^{*}<h_{p c}$ region. The state-space distributions of $C_{p}{ }^{*}$,

Table 6-2: The critical pressure and temperature of fluids commonly used in heat transfer studies involving supercritical thermophysical states.

Fluid Critical pressure, $P_{c},(\mathrm{MPa}) \quad$ Critical temperature, $T_{c},(\mathrm{~K})$

$\begin{array}{lcr}\text { Water }\left(\mathrm{H}_{2} \mathrm{O}\right) & 22.064 & 647.10 \\ \text { Carbon dioxide }\left(\mathrm{CO}_{2}\right) & 7.380 & 304.19 \\ \text { Helium }(\mathrm{He}) & 0.224 & 4.99 \\ \mathrm{R} 134 \mathrm{a}\left(\mathrm{CH}_{2} \mathrm{FCF}_{3}\right) & 4.059 & 374.21\end{array}$



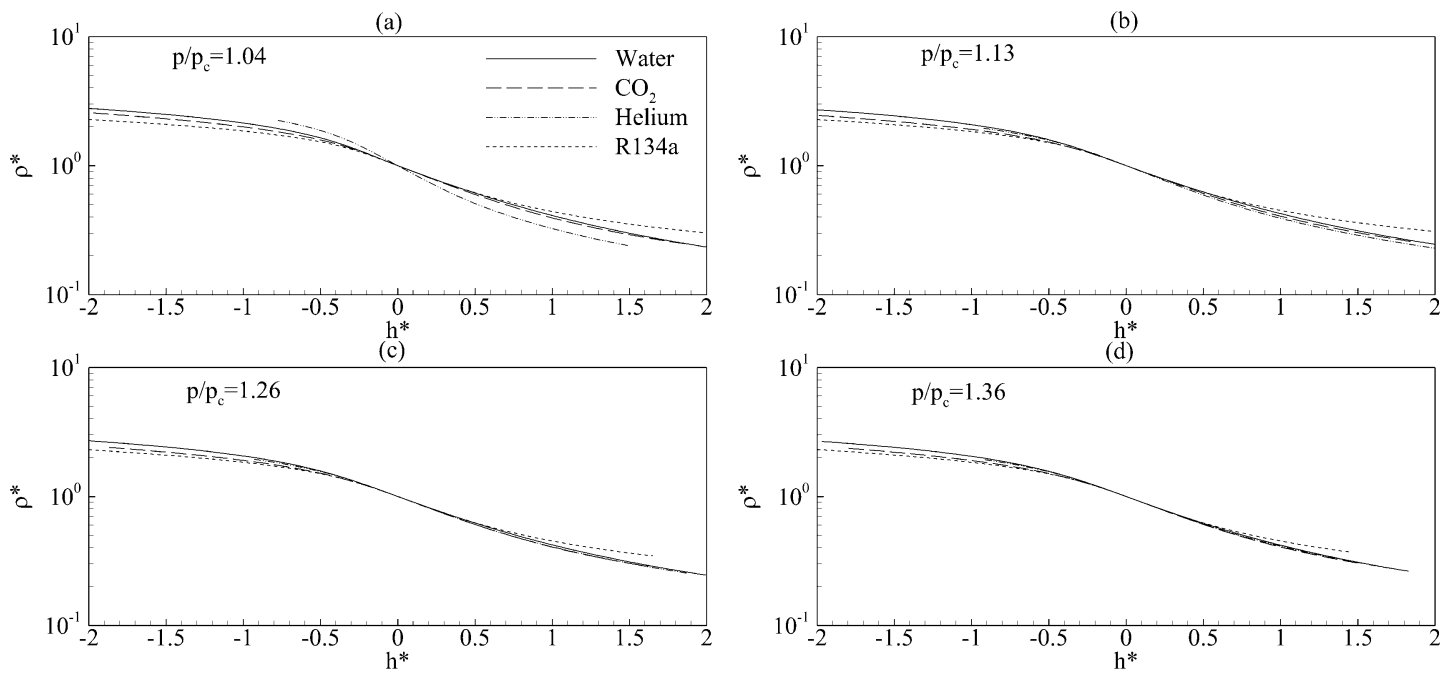

Figure 6-1: Variation of $\rho^{*}$ with $h^{*}$ for water, carbon dioxide, helium, and R134a in the vicinity of the pseudo-critical state. Data obtained from the NIST Standard Reference Database (Lemmon, 2011).
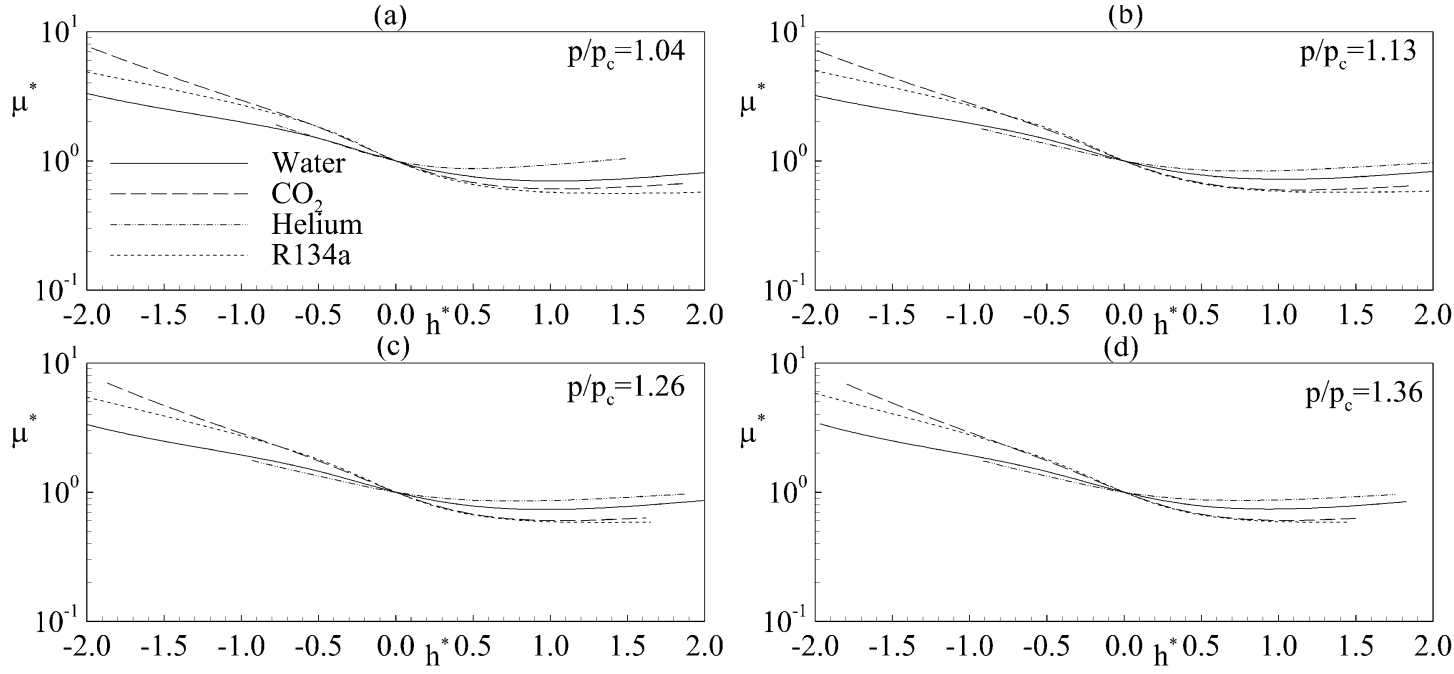

Figure 6-2: Variation of $\mu^{*}$ with $h^{*}$ for water, carbon dioxide, helium, and R134a in the vicinity of the pseudo-critical state. Data obtained from the NIST Standard Reference Database (Lemmon, 2011). 

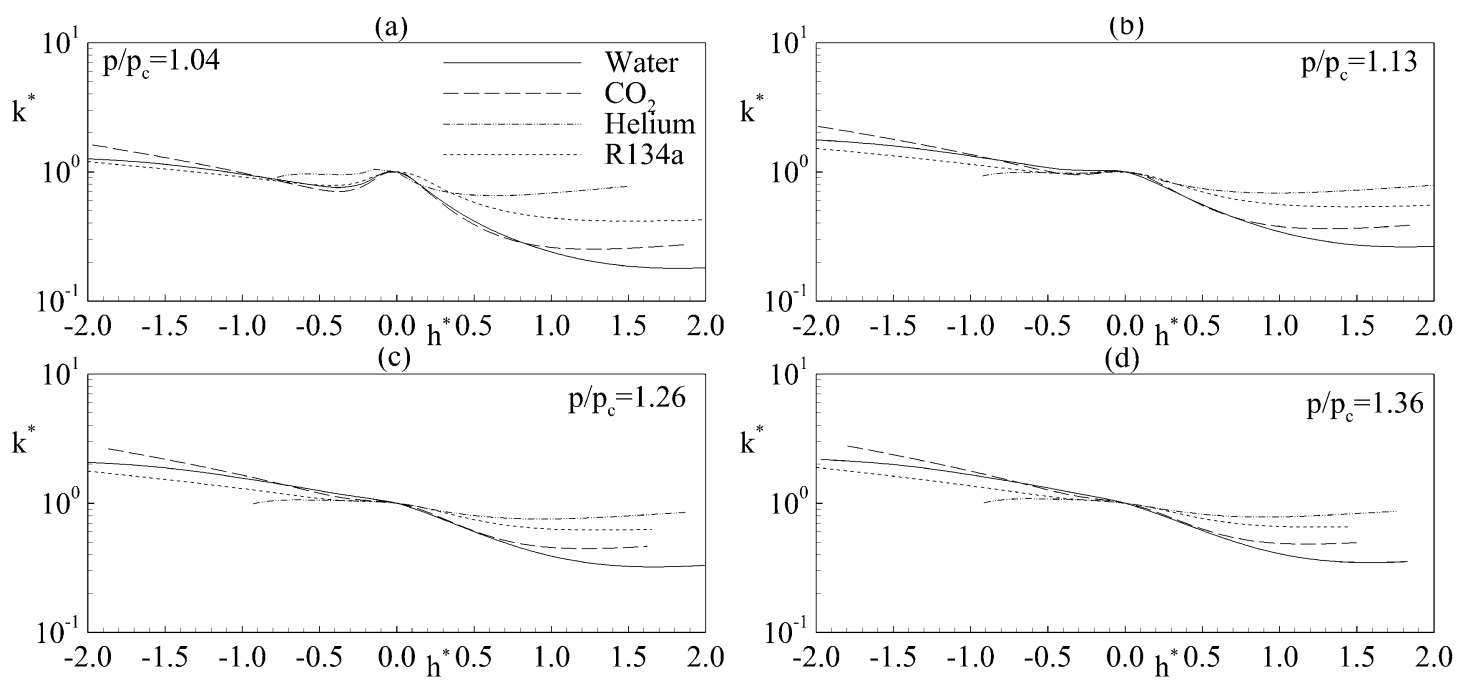

Figure 6-3: Variation of $k^{*}$ with $h^{*}$ for water, carbon dioxide, helium, and R134a in the vicinity of the pseudo-critical state. Data obtained from the NIST Standard Reference Database (Lemmon, 2011).
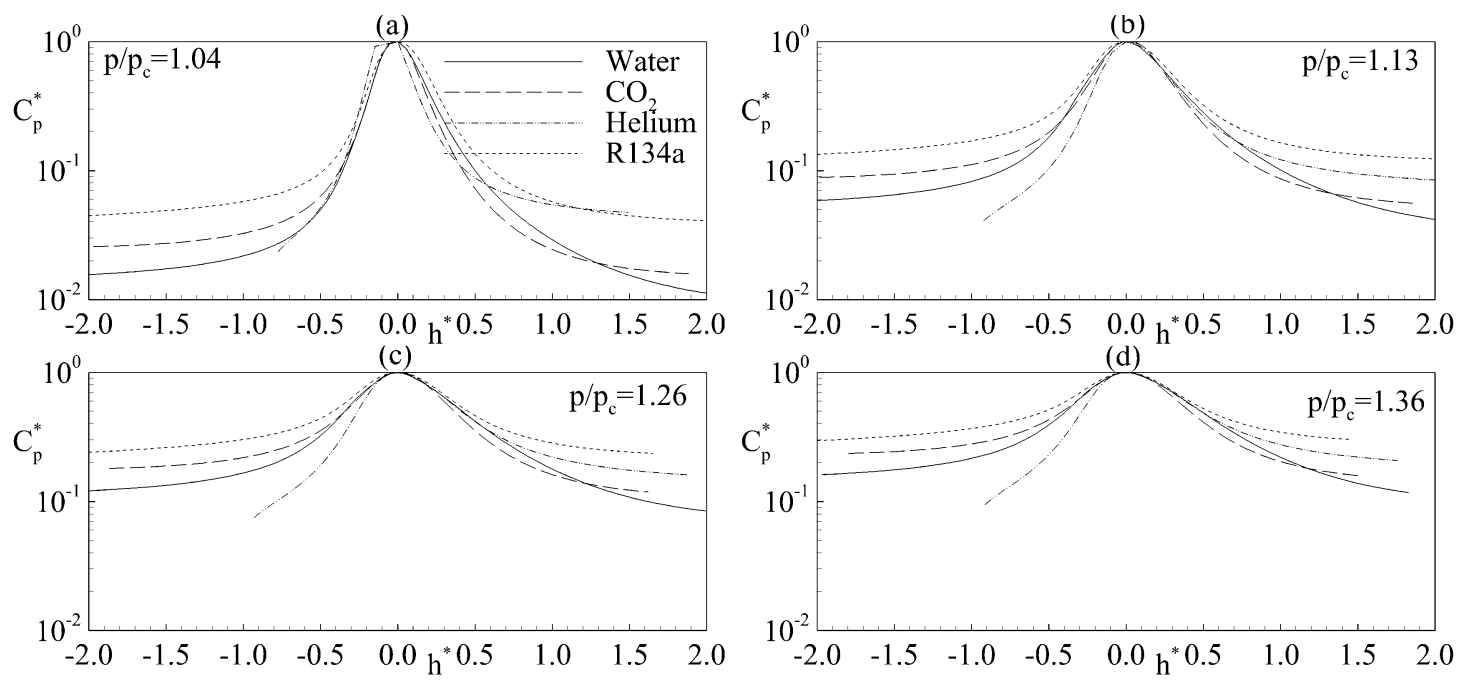

Figure 6-4: Variation of $C_{p}{ }^{*}$ with $h^{*}$ for water, carbon dioxide, helium, and R134a in the vicinity of the pseudo-critical state. Data obtained from the NIST Standard Reference Database (Lemmon, 2011).

shown in Figure 6-4, are most similar for water and carbon dioxide, particularly in the $h^{*}>h_{p c}$ region. These distributions for helium are observed to deviate most significantly from the other three fluids in the $h^{*}<h_{p c}$ region. Overall, the dependencies of $\rho^{*}, \mu^{*}, k^{*}$, 
and $C_{p}{ }^{*}$ on $h^{*}$ for the four analyzed fluids indicates that the behaviour of helium is most dissimilar from the remaining three fluids. Figure 6-5 compares the state-space distributions of $\rho, \mu, C_{p}$, and $k$ for water, carbon dioxide, helium, and R134a at $p / p_{c}=1.13$ using several independent non-dimensional parameters, namely: $\left.h^{*}=\beta_{p c} / C_{p, p c}\right)\left(h-h_{p c}\right)$ as proposed in the present study; $T / T_{p c}$ proposed by Bogachev et al. (1984), and $\left(T-T_{p c}\right) /\left(T_{p c}-T_{c}\right)$ proposed by Cheng et al. (2011). It is readily observed from
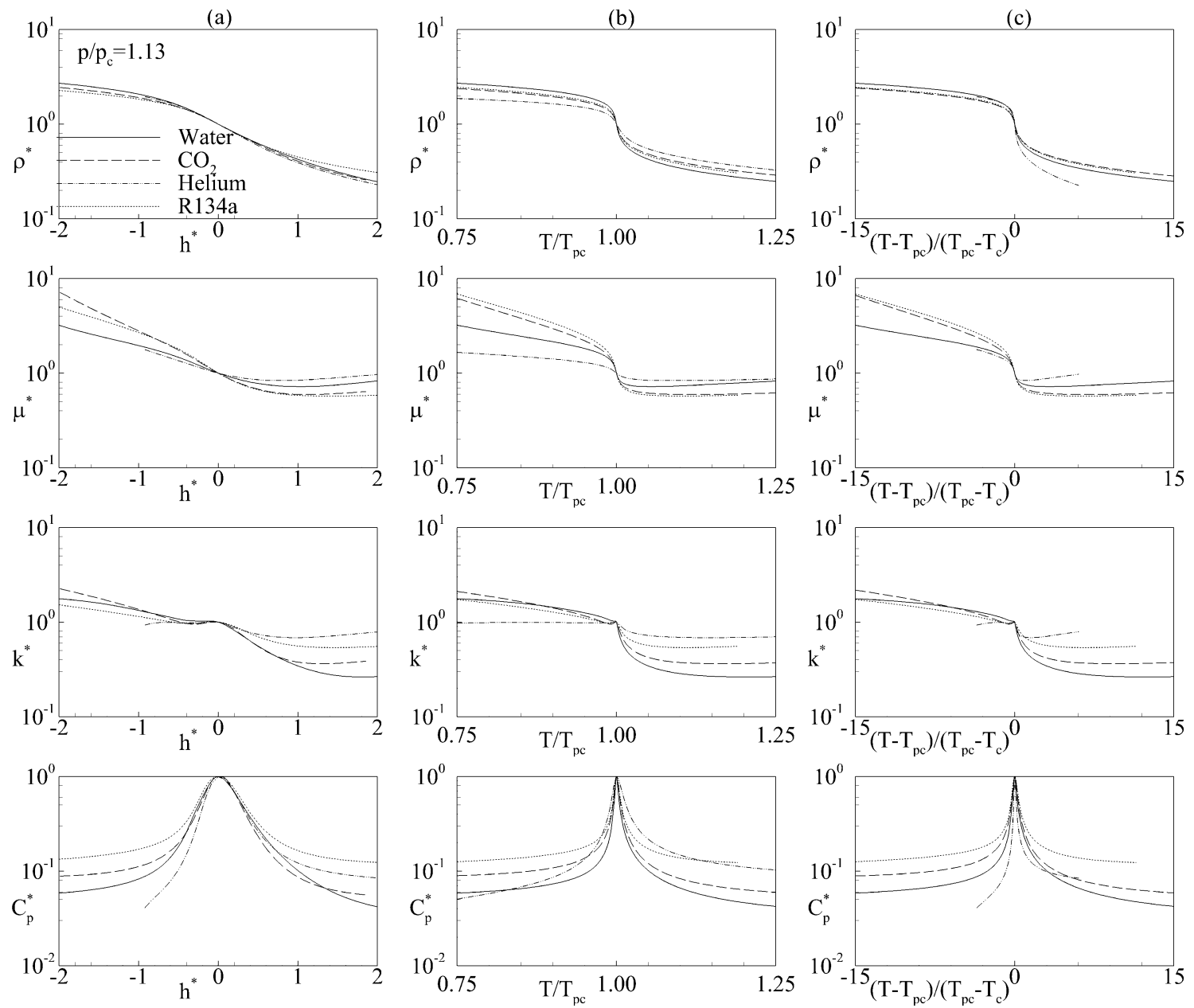

Figure 6-5: State-space distribution of thermophysical properties based on three nondimensional independent state-space parameters: (a) $h^{*}=\left(\beta_{p c} / C_{p, p c}\right)\left(h-h_{p c}\right)$; (b) $T / T_{p c}$; (c) $\left(T-T_{p c}\right) /\left(T_{p c}-T_{c}\right)$. Data obtained from the NIST Standard Reference Database (Lemmon, 2011). 
this figure that $T / T_{p c}$ performs less favourably than $h^{*}$ and $\left(T-T_{p c}\right) /\left(T_{p c}-T_{c}\right)$ in establishing similarity in state space. Advantages of $h^{*}$ over $\left(T-T_{p c}\right) /\left(T_{p c}-T_{c}\right)$ are noted in the state-space distributions of density, observed through the more favourable agreement of the density distribution for helium with those of the remaining three fluids. As previously stated, variations in the value of density is of particular interest for mixedconvection heat transfer in supercritical fluids. It is also reemphasized that the temperature ratio, $\left(T-T_{p c}\right) /\left(T_{p c}-T_{c}\right)$, is singular at the critical state and sensitive to small uncertainties in the state variables near the critical state.

The variation of the Prandtl number with $h^{*}$ is illustrated in Figure 6-6. Amongst the four analyzed fluids, the variations of $\operatorname{Pr}$ with $h^{*}$ of carbon dioxide and R134a are particularly in good agreement with each other. It is also noted from Figure 6-6 that for $p / p_{c} \geq 1.13$ the
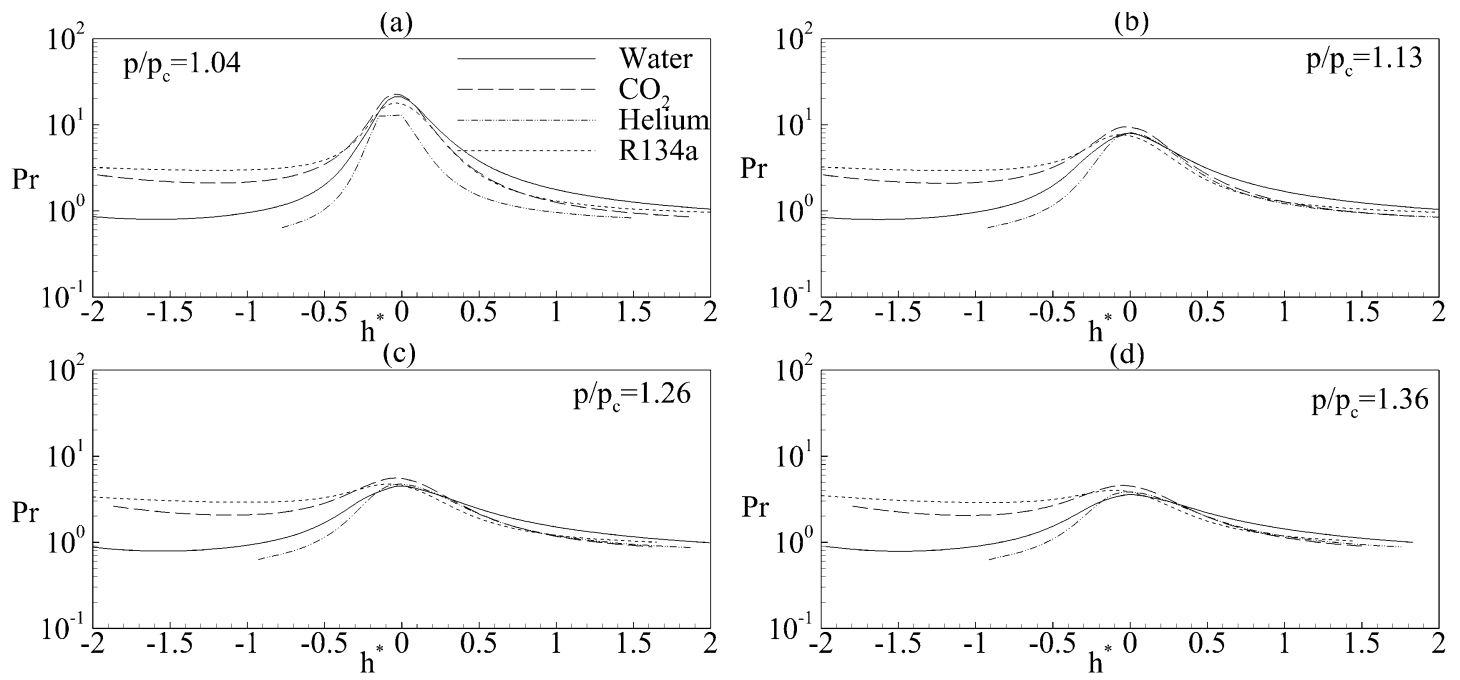

Figure 6-6: Variation of $\operatorname{Pr}$ with $h^{*}$ for water, carbon dioxide, helium, and R134a. Data obtained from the NIST Standard Reference Database (Lemmon, 2011). 
distributions of $\operatorname{Pr}$ for water and helium compare favourably. In subcritical single-phase flows, differences in the $\operatorname{Pr}$ distributions are known to yield differences in the heat transfer characteristics. For example, a difference in the value of $\operatorname{Pr}$ by a factor of 10 between two cases of convection heat transfer would yield a difference in the magnitude of $N u$ by a factor of $\sim 2$ (Winterton, 1998). However, it will be demonstrated in Section 6.5 that IHT and DHT conditions can be consistently predicted for ducted flows of supercritical fluids despite the differences in $\operatorname{Pr}$ distributions for the four analyzed fluids observed in Figure 6-6.

\subsection{Similarity Criteria for Mixed-Convection Heat Transfer in Ducted Flows of Supercritical Fluids}

Based on the discussions presented in Sections 6.2 and 6.3, a set of similarity criteria to support a fluid-to-fluid model is proposed for mixed-convection heat transfer in ducted flows of supercritical fluids. The duct geometry is selected based on the availability of sufficient experimental data to adequately validate the proposed model. As per Equations 6-26b and 6-27, $Q^{*}$ represents the change in $h^{*}$ along the flow path of the heated duct. Therefore, for separate setups with different working fluids, if the fluids are sufficiently similar in state space based on the analyses presented in Section 6.3, for a given inflow value of $h^{*}$ and $p^{*}=p / p_{c}$, then the distribution of state variables $\rho^{*}, \mu^{*}, C_{p}{ }^{*}$ and $k^{*}$ along the flow path would be similar provided that $Q^{*}$ is matched between the setups. To ensure consistency with the analyses in Section 6.3 , the $Q^{*}$ expression in 
Equation 6-27 is rewritten with the pseudo-critical state taken as the reference state of the fluid, such that:

$$
Q^{*}=\beta_{p c} / C_{p, p c}\left(\dot{Q}_{w}^{\prime \prime} / G\right)
$$

As per the discussion in Section $6.2 R e, R i^{\prime}$, and $\operatorname{Pr}$ must also be distributed similarly along the flow path in the subject setups. Similarity in the $\operatorname{Pr}$ value is ensured once similarity is realized for the thermophysical parameters by matching $h_{i n}^{*}, p_{i n}^{*}$, and $Q^{*}$ between the two setups. Similarity in the distributions of $R e$ and $R i^{\prime}$ is satisfied if their respective values are similar at the inflow and evolve in a similar manner along the flow path. These streamwise evolutions of $R e$ and $R i^{\prime}$ from their respective inflow values are governed by the distributions of the thermophysical properties along the flow path, the similarity of which are established by matching $Q^{*}$ and the inflow values of $h^{*}$ and $p^{*}$. Thus, for a model setup and prototype setup involving ducted flow of supercritical fluids, respectively indicated by the subscripts $M$ and $P$, the proposed similarity criteria involving ducted flow of supercritical fluids comprises of the following parameters:

$$
\begin{aligned}
\left\{p_{i n}^{*}\right\}_{M} & =\left\{p_{i n}^{*}\right\}_{P} \\
\left\{h_{i n}^{*}\right\}_{M} & =\left\{h_{i n}^{*}\right\}_{P} \\
\left\{R e_{i n}\right\}_{M} & =\left\{R e_{i n}\right\}_{P} \\
\left\{R i_{i n}^{\prime}\right\}_{M} & =\left\{R i_{i n}^{\prime}\right\}_{P} \\
\left\{Q^{*}\right\}_{M} & =\left\{Q^{*}\right\}_{P}
\end{aligned}
$$

where, 


$$
\begin{gathered}
p_{i n}^{*}=\frac{p_{i n}}{p_{c}} \\
h_{i n}^{*}=\frac{\beta_{p c}}{C_{p, p c}}\left(h_{i n}-h_{p c}\right) \\
R e_{i n}=\frac{G d_{h}}{\mu_{i n}} \\
R i^{\prime}=\frac{\rho_{\text {in }}^{2} g d_{h}^{2}}{G^{2}} \frac{\beta_{i n} \dot{Q}_{w}^{\prime \prime}}{k_{i n}} \\
Q^{*}=\beta_{p c} / C_{p, p c}\left(\dot{Q}_{w}^{\prime \prime} / G\right)
\end{gathered}
$$

Equation 6-14(d) is adopted as the expression for the Reynolds number, where the velocity ratio $\left(u_{I} / u_{\mu}\right)$ appearing in the definition of the Reynolds number in Equation 6-14(d) is omitted. The use of Equation 6-14(d) is justified since this velocity ratio is expected to be similar once similarity in the non-dimensional thermophysical parameters and in $Q^{*}$ is ensured. The similarity in this velocity ratio also justifies conversion of the proportionality to an equality in Equation 6-14(d). The proportionality in the expression of the Richardson number in Equation 6-22 arises from the definitions of the reference densities in Equations 6-15 and 6-18(b). The inflow density is selected as the reference density to ensure a value of zero for the buoyancy force prior to heating the flow, as per Equation 6-15. Once similarity in the inflow state and rate of heating are established by matching $h_{i n}^{*}, p_{i n}^{*}$, and $Q^{*}$ the proportionality constant is expected to be similar for the model and prototype setups and can thus be omitted from the expression for $R i^{\prime}$ based on Equation 6-22. The hydraulic diameter, $d_{h}$, is specified as the reference length scale for $R e_{i n}$ and $R i_{i n}^{\prime}$, and is well established as a relevant length scale for mixed-convection heat transfer in ducted flows (Sobhan \& Garimella, 2001). 


\subsection{Validation of the Similarity Criteria for Heat Transfer in Supercritical Fluids}

To validate the similarity criteria presented in Equation 6-32, heat transfer data was compiled from published literature for ducted flows of water, carbon dioxide, helium, and R134a. The data compiled comprise of flows with upstream (upward flow), downstream (downward flow), and wall-normal (horizontal flow) orientated gravitational accelerations, respectively tabulated in Table 6-3 to Table 6-5. From the readily available data, only datasets with sufficient information to calculate the parameters in Equation 6-32, and sufficient heated lengths to characterize the IHT and DHT signatures were analyzed. These signatures, discussed in Section 2.2, were evident in the distributions of the wall temperature and Nusselt number along the flow path. Here, the Nusselt number is computed through the expression in Equation 6-21, with the assumptions that $\Delta T_{B}$ and $\Delta T_{k}$ are of comparable magnitude and $k_{w}$ can be approximated by $k_{b}$. The latter assumption is a necessary simplification since the value of $k_{w}$ cannot be reliably estimated a priori for heat transfer in flows of supercritical fluids, and ensures consistency with the published categorization of flows exhibiting IHT and DHT phenomena. 
Table 6-3: Values of the similarity criteria for ducted flows with upstream-oriented gravitational acceleration.

Author

(Source, if different Fluid

$$
p^{*} \quad h_{i n}^{*} \quad R e_{i n} \quad R i_{i n}^{\prime} \quad Q^{*}
$$

from Author)

Ackerman (1970)

$\mathrm{H}_{2} \mathrm{O}$

$1.03-0.8$

$3.0 \mathrm{E} 4$

$1.3 \mathrm{E}-1$

$6.5 \mathrm{E}-4$

to 1.41 to -2.9 to $1.5 \mathrm{E} 5$ to $3.5 \mathrm{E} 1$ to $1.8 \mathrm{E}-3$

Alekseev et al. (1976)

(Pioro \& Duffey,

2005)

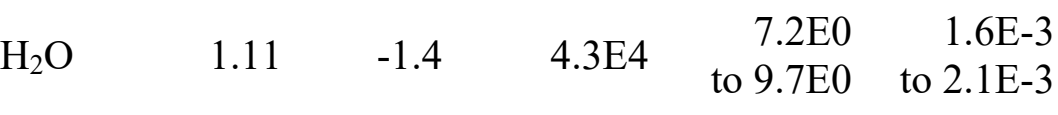

Herkerath (1970)

Krau et al. (2008)

$\begin{array}{llllr} & & & & \\ \mathrm{H}_{2} \mathrm{O} & 1.02 & -0.8 & 1.9 \mathrm{E} 5 & \begin{array}{r}1.6 \mathrm{E}-1 \\ \text { to } 1.1 \mathrm{E} 0\end{array} \text { to } 9.14 \mathrm{E}-4\end{array}$

Kirillov et al. (1986)

(Pioro \& Duffey, 2005)

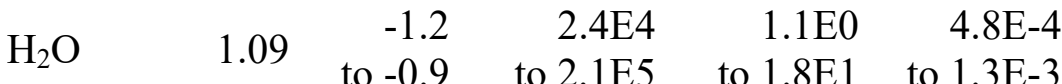

Lee \& Haller (1974)

(Pioro \& Duffey,

2005)

Shitsman (1963)

$\mathrm{H}_{2} \mathrm{O}$

1.05 to -1.2
1.15

$4.1 \mathrm{E} 4$

to $1.8 \mathrm{E} 1$ to $1.3 \mathrm{E}-3$

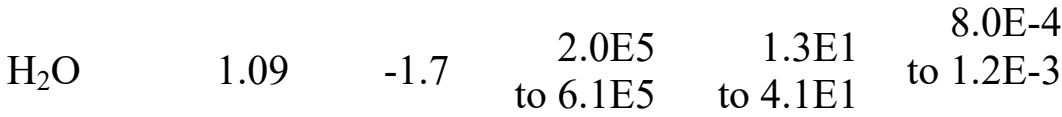

Vikhrev et al. (1967)

(Pioro \& Duffey,

2005)

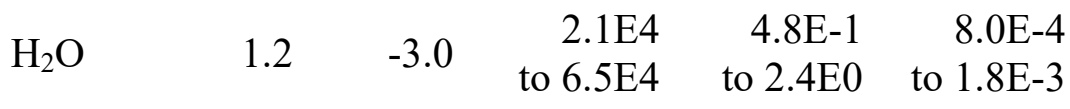

Watts (2009)

$\mathrm{H}_{2} \mathrm{O}$

$1.13-2.5$

$2.1 \mathrm{E} 4$

$1.1 \mathrm{E} 1$

$8.4 \mathrm{E} 4$

to $8.4 \mathrm{E} 4$ to $1.6 \mathrm{E} 2$ to $3.3 \mathrm{E} 3$

Yamagata et al.

(1972)

$\mathrm{H}_{2} \mathrm{O}$

$1.02-1.8$

1.1E5

$3.5 \mathrm{E}-1$

3.0E-4 to 1.33 to -1.4 to $1.3 \mathrm{E} 5$ to $1.4 \mathrm{E} 0$ to $1.3 \mathrm{E}-3$

Yu et al. (2013)

$\mathrm{H}_{2} \mathrm{O}$

$1.04-1.8$

$1.5 \mathrm{E} 5$

8.3E0 $\quad 5.8 \mathrm{E}-4$ to $1.2 \mathrm{E} 1$ to $8.6 \mathrm{E}-4$

Zhang et al. (2012) $\quad \mathrm{H}_{2} \mathrm{O}$

$$
1.04 \quad-1.3 \quad 6.6 \mathrm{E} 4 \quad 1.1 \mathrm{E} 0 \quad 1.1 \mathrm{E}-3
$$

to 1.13 to -1.2 to $2.4 \mathrm{E} 5$ to $6.3 \mathrm{E} 0$ to $2.2 \mathrm{E}-3$

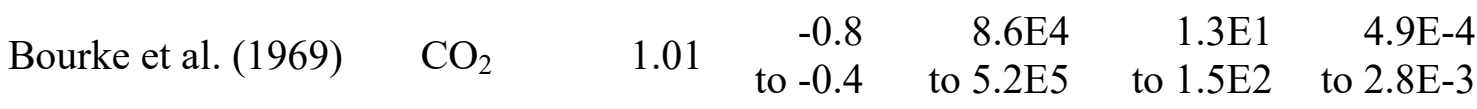




\begin{tabular}{|c|c|c|c|c|c|c|}
\hline $\begin{array}{l}\text { Author } \\
\text { (Source, if different } \\
\text { from Author) }\end{array}$ & Fluid & $p^{*}$ & $h_{i n}^{*}$ & $R e_{i n}$ & $R i_{i n}^{\prime}$ & $Q^{*}$ \\
\hline Bae et al. (2010) & $\mathrm{CO}_{2}$ & $\begin{array}{r}1.05 \\
\text { to } 1.10\end{array}$ & -1.1 & $\begin{array}{r}1.7 \mathrm{E} 4 \\
\text { to } 3.5 \mathrm{E} 4\end{array}$ & $\begin{array}{r}3.1 \mathrm{E}-1 \\
\text { to } 4.0 \mathrm{E} 0\end{array}$ & $\begin{array}{r}6.2 \mathrm{E}-4 \\
\text { to } 1.5 \mathrm{E}-3\end{array}$ \\
\hline $\begin{array}{l}\text { Fewster (1976) } \\
\text { (Duffey \& Pioro, } \\
2005)\end{array}$ & $\mathrm{CO}_{2}$ & 1.03 & $\begin{array}{r}-0.7 \\
\text { to }-0.4\end{array}$ & $\begin{array}{r}4.2 \mathrm{E} 4 \\
\text { to } 1.0 \mathrm{E} 5\end{array}$ & $\begin{array}{r}1.2 \mathrm{E} 0 \\
\text { to } 1.4 \mathrm{E} 2\end{array}$ & $\begin{array}{l}6.7 \mathrm{E}-4 \\
8.6 \mathrm{E}-4\end{array}$ \\
\hline Gupta et al. (2013) & $\mathrm{CO}_{2}$ & $\begin{array}{r}1.04 \\
\text { to } 1.19\end{array}$ & $\begin{array}{r}-0.4 \\
\text { to }-0.6\end{array}$ & $1.1 \mathrm{E} 5$ & $\begin{array}{r}7.5 \mathrm{e}-1 \\
\text { to } 8.9 \mathrm{E}-1\end{array}$ & $\begin{array}{l}1.9 \mathrm{E}-4 \\
2.3 \mathrm{E}-4\end{array}$ \\
\hline Jiang et al. (2008) & $\mathrm{CO}_{2}$ & 1.17 & -0.5 & $2.0 \mathrm{E} 3$ & $\begin{array}{r}1.9 \mathrm{E}-3 \\
\text { to } 1.9 \mathrm{E}-2\end{array}$ & $\begin{array}{r}2.2 \mathrm{E}-4 \\
\text { to } 2.2 \mathrm{E}-3\end{array}$ \\
\hline Kim \& Kim (2011) & $\mathrm{CO}_{2}$ & $\begin{array}{r}1.02 \\
\text { to } 1.23\end{array}$ & $\begin{array}{r}-0.5 \\
\text { to }-0.3\end{array}$ & $\begin{array}{r}1.7 \mathrm{E} 4 \\
\text { to } 8.1 \mathrm{E} 4\end{array}$ & $\begin{array}{r}2.6 \mathrm{E} 0 \\
\text { to } 1.3 \mathrm{E} 1\end{array}$ & $\begin{array}{r}8.8 \mathrm{E}-4 \\
\text { to } 2.9 \mathrm{E}-3\end{array}$ \\
\hline Li et al. (2010) & $\mathrm{CO}_{2}$ & 1.19 & -0.7 & $9.0 \mathrm{E} 3$ & $\begin{array}{r}3.9 \mathrm{E}-1 \\
\text { to } 7.6 \mathrm{E}-1\end{array}$ & $\begin{array}{r}1.0 \mathrm{E}-3 \\
\text { to } 1.3 \mathrm{E}-3\end{array}$ \\
\hline Petukhov et al. (1983) & $\mathrm{CO}_{2}$ & $\begin{array}{r}1.04 \\
\text { to } 1.12\end{array}$ & $\begin{array}{l}-1.0 \\
\text { to }- \\
0.47\end{array}$ & $\begin{array}{r}2.66 \mathrm{E} 5 \\
\text { to } 4.57 \mathrm{E} 5\end{array}$ & $\begin{array}{r}5.3 \mathrm{E}-1 \\
\text { to } 2.9 \mathrm{E} 0\end{array}$ & $\begin{array}{r}9.1 \mathrm{E}-4 \\
\text { to } 2.2 \mathrm{E}-3\end{array}$ \\
\hline $\begin{array}{l}\text { Shiralkar \& Griffith } \\
\text { (1968) }\end{array}$ & $\mathrm{CO}_{2}$ & 1.03 & $\begin{array}{r}-0.9 \\
\text { to }-0.5\end{array}$ & $\begin{array}{r}1.1 \mathrm{E} 5 \\
\text { to } 1.9 \mathrm{E} 5\end{array}$ & $\begin{array}{r}2.2 \mathrm{E}-1 \\
\text { to } 3.5 \mathrm{E} 0\end{array}$ & $\begin{array}{r}8.7 \mathrm{E}-4 \\
\text { to } 1.1 \mathrm{E}-3\end{array}$ \\
\hline Song et al. (2008) & $\mathrm{CO}_{2}$ & 1.10 & $\begin{array}{r}-1.1 \\
\text { to }-0.4\end{array}$ & $\begin{array}{r}1.7 \mathrm{E} 4 \\
\text { to } 2.2 \mathrm{E} 5\end{array}$ & $\begin{array}{r}1.6 \mathrm{E}-1 \\
\text { to } 1.1 \mathrm{E} 1\end{array}$ & $\begin{array}{r}3.5 \mathrm{E}-4 \\
\text { to } 1.1 \mathrm{E}-3\end{array}$ \\
\hline $\begin{array}{l}\text { Weinberg (1972) } \\
\text { (Duffey \& Pioro, } \\
\text { 2005) }\end{array}$ & $\mathrm{CO}_{2}$ & 1.03 & -0.7 & $1.1 \mathrm{E} 5$ & $\begin{array}{r}1.8 \mathrm{E} 1 \\
\text { to } 3.1 \mathrm{E} 1\end{array}$ & $9.6 \mathrm{E}-4$ \\
\hline $\begin{array}{l}\text { Bogachev et al. } \\
\text { (1984) }\end{array}$ & $\mathrm{He}$ & 1.10 & -0.8 & $2.4 \mathrm{E} 4$ & $\begin{array}{r}1.3 \mathrm{E} 0 \\
\text { to } 2.6 \mathrm{E} 0\end{array}$ & $\begin{array}{r}8.2 \mathrm{E}-4 \\
\text { to } 1.7 \mathrm{E}-3\end{array}$ \\
\hline $\begin{array}{l}\text { Brassington \& Carins } \\
\text { (1977) }\end{array}$ & $\mathrm{He}$ & $\begin{array}{r}1.02 \\
\text { to } 1.41\end{array}$ & $\begin{array}{l}-0.5 \\
-0.7\end{array}$ & $\begin{array}{r}2.3 \mathrm{E} 5 \\
\text { to } 5.4 \mathrm{E} 5\end{array}$ & $\begin{array}{r}2.0 \mathrm{E} 1 \\
\text { to } 1.6 \mathrm{E} 2\end{array}$ & $\begin{array}{r}2.4 \mathrm{E}-4 \\
\text { to } 1.1 \mathrm{E}-3\end{array}$ \\
\hline $\begin{array}{l}\text { Giarratano et al. } \\
\text { (1971) }\end{array}$ & $\mathrm{He}$ & $\begin{array}{r}1.41 \\
\text { to } 1.85\end{array}$ & $\begin{array}{r}-0.7 \\
\text { to }-0.3\end{array}$ & $\begin{array}{r}3.8 \mathrm{E} 4 \\
\text { to } 3.0 \mathrm{E} 5\end{array}$ & $\begin{array}{r}3.9 \mathrm{E}-2 \\
\text { to } 1.5 \mathrm{E} 1\end{array}$ & $\begin{array}{r}1.7 \mathrm{E}-4 \\
\text { to } 8.9 \mathrm{E}-4\end{array}$ \\
\hline
\end{tabular}




\begin{tabular}{|c|c|c|c|c|c|c|}
\hline $\begin{array}{l}\text { Author } \\
\text { (Source, if different } \\
\text { from Author) }\end{array}$ & Fluid & $p^{*}$ & $h_{i n}^{*}$ & $R e_{i n}$ & $R i_{\text {in }}^{\prime}$ & $Q^{*}$ \\
\hline Kang \& Chang (2009) & R134a & 1.06 & -1.8 & $3.7 \mathrm{E} 4$ & $\begin{array}{r}5.3 \mathrm{E}-1 \\
\text { to } 3.2 \mathrm{E} 0\end{array}$ & $\begin{array}{r}2.5 \mathrm{E}-4 \\
\text { to } 1.5 \mathrm{E}-3\end{array}$ \\
\hline Zhang (2014) & R134a & 1.06 & $\begin{array}{r}-1.3 \\
\text { to }-0.4\end{array}$ & $\begin{array}{r}3.94 \mathrm{E} 4 \\
\text { to } 2.7 \mathrm{E} 5\end{array}$ & $\begin{array}{r}9.5 \mathrm{E}-1 \\
\text { to } 7.4 \mathrm{E} 0\end{array}$ & $\begin{array}{r}4.0 \mathrm{E}-4 \\
\text { to } 1.2 \mathrm{E} 3\end{array}$ \\
\hline
\end{tabular}

Table 6-4: Values of the similarity criteria for ducted flows with downstream-oriented gravitational acceleration.

\begin{tabular}{|c|c|c|c|c|c|c|}
\hline $\begin{array}{l}\text { Author } \\
\text { (Source, if different } \\
\text { from Author) }\end{array}$ & Fluid & $p^{*}$ & $h_{i n}^{*}$ & $R e_{i n}$ & $R i_{\text {in }}^{\prime}$ & $Q^{*}$ \\
\hline $\begin{array}{l}\text { Krasyakova et al. } \\
\text { (1977) (Pioro \& } \\
\text { Duffey, 2005) }\end{array}$ & $\mathrm{H}_{2} \mathrm{O}$ & 1.11 & -2.2 & $1.4 \mathrm{E} 5$ & $\begin{array}{r}2.5 \mathrm{E} 0 \\
\text { to } 4.4 \mathrm{E} 0\end{array}$ & $\begin{array}{r}7.7 \mathrm{E}-4 \\
\text { to } 2.0 \mathrm{E}-3\end{array}$ \\
\hline Watts (2009) & $\mathrm{H}_{2} \mathrm{O}$ & 1.13 & -2.5 & $\begin{array}{r}2.6 \mathrm{E} 4 \\
\text { to } \\
8.6 \mathrm{E} 4\end{array}$ & $\begin{array}{r}3.1 \mathrm{E} 0 \\
\text { to } 3.1 \mathrm{E} 1\end{array}$ & $\begin{array}{r}4.7 \mathrm{E}-4 \\
\text { to } 1.5 \mathrm{E}-3\end{array}$ \\
\hline Bourke et al. (1969) & $\mathrm{CO}_{2}$ & 1.01 & -0.8 & $3.4 \mathrm{E} 5$ & $\begin{array}{r}2.1 \mathrm{E} 1 \\
\text { to } 2.7 \mathrm{E} 1\end{array}$ & $\begin{array}{r}1.5 \mathrm{E}-3 \\
\text { to } 1.9 \mathrm{E}-3\end{array}$ \\
\hline Kim \& Kim (2011) & $\mathrm{CO}_{2}$ & $\begin{array}{r}1.01 \\
\text { to } 1.10\end{array}$ & $\begin{array}{r}-0.5 \\
\text { to }-0.4\end{array}$ & $\begin{array}{r}4.9 \mathrm{E} 4 \\
\text { to } \\
1.9 \mathrm{E} 5\end{array}$ & $\begin{array}{r}2.7 \mathrm{E} 0 \\
\text { to } 1.1 \mathrm{E} 1\end{array}$ & $\begin{array}{r}1.7 \mathrm{E}-3 \\
\text { to } 2.1 \mathrm{E}-3\end{array}$ \\
\hline Petukhov et al. (1983) & $\mathrm{CO}_{2}$ & $\begin{array}{r}1.04 \\
\text { to } 1.12\end{array}$ & $\begin{array}{r}-0.5 \\
\text { to }-0.7\end{array}$ & $\begin{array}{r}3.5 \mathrm{E} 5 \\
\text { to } \\
4.8 \mathrm{E} 5\end{array}$ & $\begin{array}{r}7.9 \mathrm{E}-1 \\
\text { to } 3.0 \mathrm{E} 0\end{array}$ & $\begin{array}{r}9.5 \mathrm{E}-4 \\
\text { to } 2.6 \mathrm{E}-3\end{array}$ \\
\hline $\begin{array}{l}\text { Shiralkar \& Griffith } \\
\text { (1968) }\end{array}$ & $\mathrm{CO}_{2}$ & 1.03 & -0.9 & $\begin{array}{r}1.1 \mathrm{E} 5 \\
\text { to } \\
1.4 \mathrm{E} 5\end{array}$ & $\begin{array}{r}5.1 \mathrm{E}-1 \\
\text { to } 8.9 \mathrm{E}-1\end{array}$ & $\begin{aligned} & 8.2 \mathrm{E}-4 \\
& \text { to } 1.1 \mathrm{E}-3\end{aligned}$ \\
\hline $\begin{array}{l}\text { Weinberg (1972) } \\
\text { (Duffey \& Pioro, 2005) }\end{array}$ & $\mathrm{CO}_{2}$ & 1.03 & -0.7 & $1.1 \mathrm{E} 5$ & $\begin{array}{r}1.8 \mathrm{E} 1 \\
\text { to } 3.7 \mathrm{E} 1\end{array}$ & $\begin{array}{r}9.6 \mathrm{E}-4 \\
\text { to } 1.7 \mathrm{E}-3\end{array}$ \\
\hline Bogachev et al. (1984) & $\mathrm{He}$ & 1.10 & -0.8 & $2.4 \mathrm{E} 4$ & $\begin{array}{r}2.1 \mathrm{E} 0 \\
\text { to } 2.6 \mathrm{E} 0\end{array}$ & $\begin{array}{r}1.1 \mathrm{E}-3 \\
\text { to } 1.7 \mathrm{E}-3\end{array}$ \\
\hline $\begin{array}{l}\text { Giarratano \& Jones } \\
\text { (1975) }\end{array}$ & $\mathrm{He}$ & 1.10 & $\begin{array}{r}-0.8 \\
\text { to }-0.4\end{array}$ & $\begin{array}{r}4.2 \mathrm{E} 4 \\
\text { to } \\
4.4 \mathrm{E} 4\end{array}$ & $\begin{array}{r}4.2 \mathrm{E}-1 \\
\text { to } 5.2 \mathrm{E} 0\end{array}$ & $\begin{array}{r}2.5 \mathrm{E}-4 \\
\text { to } 3.5 \mathrm{E}-3\end{array}$ \\
\hline
\end{tabular}


Table 6-5: Values of the similarity criteria for ducted flows with wall-normal-oriented gravitational acceleration.

\begin{tabular}{|c|c|c|c|c|c|c|}
\hline $\begin{array}{l}\text { Author } \\
\text { (Source, if different } \\
\text { from Author) }\end{array}$ & Fluid & $p^{*}$ & $h_{i n}^{*}$ & $R e_{i n}$ & $R i_{\text {in }}^{\prime}$ & $Q^{*}$ \\
\hline $\begin{array}{l}\text { Domin (1963) } \\
\text { (Hall et al. 1967) }\end{array}$ & $\mathrm{H}_{2} \mathrm{O}$ & $\begin{array}{r}1.05 \\
\text { to } 1.15\end{array}$ & $\begin{array}{r}-0.9 \\
\text { to } 1.2\end{array}$ & $\begin{array}{r}1.7 \mathrm{E} 3 \\
\text { to } 6.9 \mathrm{E} 4\end{array}$ & $\begin{array}{r}2.0 \mathrm{E}-1 \\
\text { to } 1.8 \mathrm{E} 1\end{array}$ & $\begin{array}{r}1.4 \mathrm{E}-3 \\
\text { to } 1.8 \mathrm{E}-3\end{array}$ \\
\hline $\begin{array}{l}\text { Schmidt (1959) } \\
\text { (Hall et al. 1967) }\end{array}$ & $\mathrm{H}_{2} \mathrm{O}$ & 1.15 & -0.9 & $4.2 \mathrm{E} 4$ & $\begin{array}{r}1.5 \mathrm{E} 0 \\
\text { to } 2.5 \mathrm{E} 0\end{array}$ & $\begin{array}{r}1.6 \mathrm{E}-3 \\
\text { to } 2.2 \mathrm{E}-3\end{array}$ \\
\hline $\begin{array}{l}\text { Vikrev \& Lokshin } \\
\text { (1964) } \\
\text { (Hall et al. 1967) }\end{array}$ & $\mathrm{H}_{2} \mathrm{O}$ & 1.15 & -0.9 & $\begin{array}{l}4.4 \mathrm{E} 4 \\
1.1 \mathrm{E} 5\end{array}$ & $\begin{array}{l}1.7 \mathrm{E} 0 \\
1.1 \mathrm{E} 1\end{array}$ & $\begin{array}{l}1.15 \mathrm{E}-3 \\
2.88 \mathrm{E}-3\end{array}$ \\
\hline Yamagata et al. (1972) & $\mathrm{H}_{2} \mathrm{O}$ & 1.11 & -1.4 & $\begin{array}{r}1.0 \mathrm{E} 5 \\
\text { to } 1.5 \mathrm{E} 5\end{array}$ & $\begin{array}{r}1.1 \mathrm{E}-1 \\
\text { to } 9.2 \mathrm{E}-1\end{array}$ & $\begin{array}{r}2.2 \mathrm{E}-4 \\
\text { to } 1.3 \mathrm{E}-3\end{array}$ \\
\hline Yu et al. (2013) & $\mathrm{H}_{2} \mathrm{O}$ & 1.13 & -1.7 & $1.5 \mathrm{E} 5$ & $\begin{array}{r}8.2 \mathrm{E} 0 \\
\text { to } 1.2 \mathrm{E} 1\end{array}$ & $\begin{array}{r}5.8 \mathrm{E}-3 \\
\text { to } 8.4 \mathrm{E}-3\end{array}$ \\
\hline Adebiyi \& Hall (1969) & $\mathrm{CO}_{2}$ & 1.03 & $\begin{array}{r}-0.9 \\
\text { to }-0.4\end{array}$ & $\begin{array}{r}2.5 \mathrm{E} 4 \\
\text { to } 1.1 \mathrm{E} 5\end{array}$ & $\begin{array}{r}5.0 \mathrm{E} 0 \\
\text { to } 9.0 \mathrm{E} 1\end{array}$ & $\begin{array}{r}1.1 \mathrm{E}-4 \\
\text { to } 1.4 \mathrm{E}-3\end{array}$ \\
\hline Petukhov et al. (1983) & $\mathrm{CO}_{2}$ & $\begin{array}{r}1.04 \\
\text { to } 1.12\end{array}$ & $\begin{array}{r}-0.5 \\
\text { to }-0.7\end{array}$ & $\begin{array}{r}3.5 \mathrm{E} 5 \\
\text { to } 4.8 \mathrm{E} 5\end{array}$ & $\begin{array}{r}7.9 \mathrm{E}-1 \\
\text { to } 2.9 \mathrm{E} 0\end{array}$ & $\begin{array}{r}9.5 \mathrm{E}-4 \\
\text { to } 2.5 \mathrm{E}-3\end{array}$ \\
\hline
\end{tabular}

The development of IHT and DHT phenomena for a given flow condition is potentially affected by each of the similarity criteria which are listed in Equation 6-32. It is plausible that some of these criteria are more influential in the development of IHT and DHT than others. It has been noted in published literature that IHT and DHT phenomena are particularly influenced by the wall-heat-flux to mass-flux ratio (Pioro ${ }^{\mathrm{b}}$ et al., 2004). The similarity criterion $Q^{*}$, presented in Equation 6-31, represents a non-dimensional form of this ratio. Indeed, $Q^{*}$ is observed to be the primary similarity criterion that affects IHT 
and DHT phenomena as per Figure 6-7. This figure plots ranges of values of the similarity criteria for the data listed in Table 6-3, and highlights the regions where the IHT and DHT phenomena are observed. The dominance of $Q^{*}$ in determining the occurrence of these phenomena is suggested through the markedly larger ranges of respective $Q^{*}$ values of the regions where only IHT and only DHT are observed and the narrower region over which both of these phenomena are observed. About $20 \%$ of the data points analyzed are noted to fall in the overlapping region of $Q^{*}$ in which both phenomena are observed, which suggests that some of the other similarity criteria may have secondary non-negligible effects. Accordingly, the data in Table 6-3 are plotted

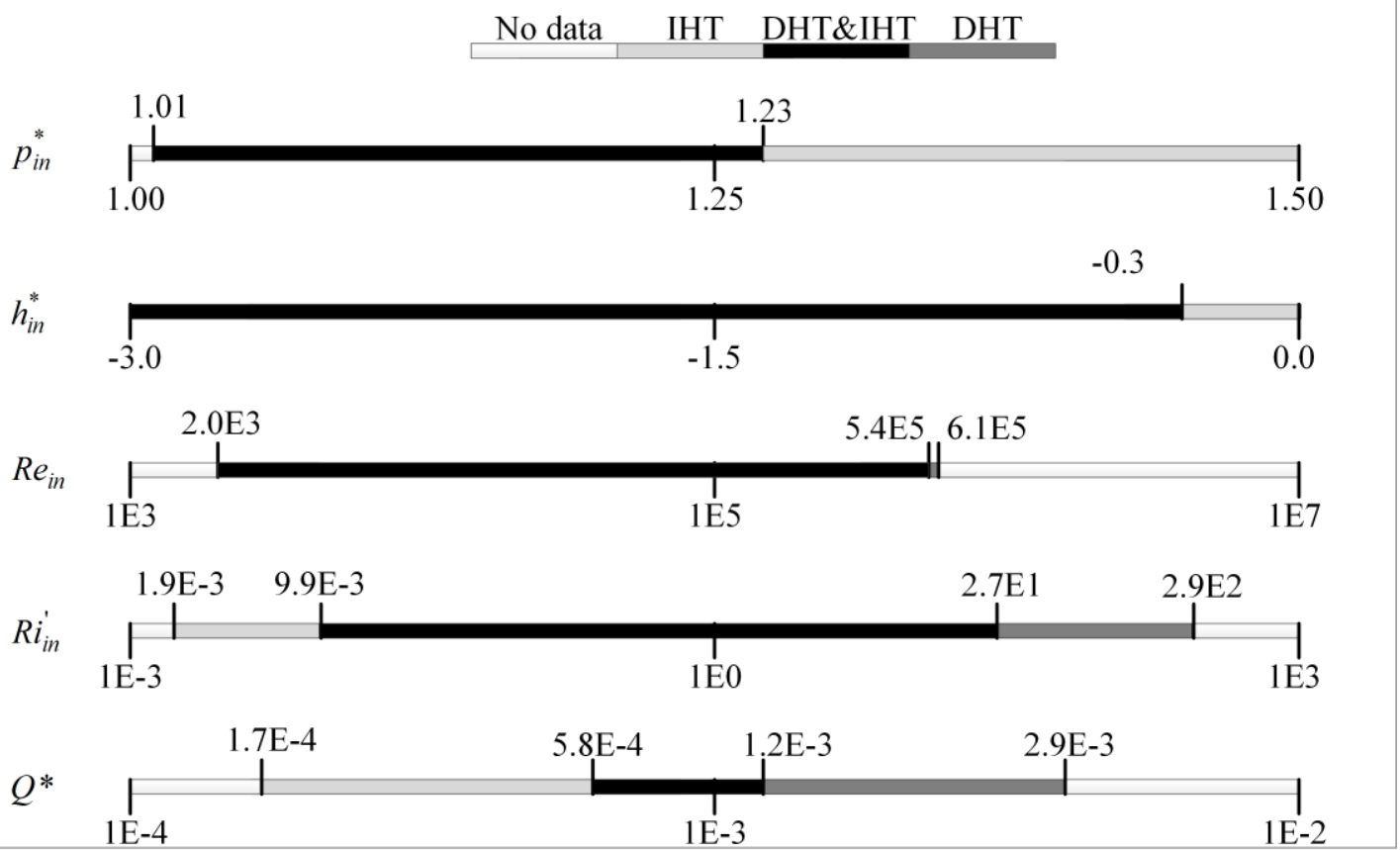

Figure 6-7: Values of similarity criteria for ducted flows with upstream-oriented gravitational acceleration, as per the studies listed in Table 6-3, showing ranges for which IHT and DHT are observed. 
against pairs of the similarity criteria to further refine the overlapping region of IHT and DHT through observations of regions where the datasets associated with IHT and DHT form clusters. These plots are shown in Figure 6-8 where $Q^{*}$ is paired with the other similarity parameters in Equation 6-32 as well as $P r_{i n}$, and $G r_{i n}$. IHT is indicated by filled symbols and DHT is indicated by hollow symbols. It is noted that amongst the plotted pairs of similarity criteria, the $p^{*}$ and $Q^{*}$ pair shows the least overlap amongst clusters of data associated with IHT and DHT, and thus most vividly captures the IHT and DHT trends. A dashed line is plotted in Figure 6-8(a) to assist in quantifying the threshold values for IHT and DHT phenomena. It is observed that $93 \%$ of the data representing IHT and DHT phenomena have values of $Q^{*} / 10^{\left(p^{*-8.58)} / 2.48\right.}$ less than and greater than unity, respectively. Some outliers to this observation are labelled A, B, and C in Figure 6-8(a). Figure 6-8(b) shows that the outlier labelled $\mathrm{A}$ is from a dataset with a relatively low value of inflow Richardson number which suggests that buoyancy forces in this instance may have affected the fluid motion to a lesser degree than in the other datasets being considered here. Datasets that cover a broader range of values for the Richardson number would likely indicate a more dominant role of this similarity criterion in determining conditions for IHT/DHT phenomena than what is suggested by the data listed in Table 6-3. The outlier labelled B is part of the dataset measured by Ackerman (1970) with water working fluid and largely falls within $10 \%$ of the threshold criterion. The outliers labelled $\mathrm{C}$ occur at relatively low values of $p^{*}\left(p^{*}<1.02\right)$ where fluctuations in the thermophysical properties of the fluid due to fluctuations in the pressure field in the flow would be particularly significant. Figure 6-9 and Figure 6-10 assess the adequacy of 
(a)

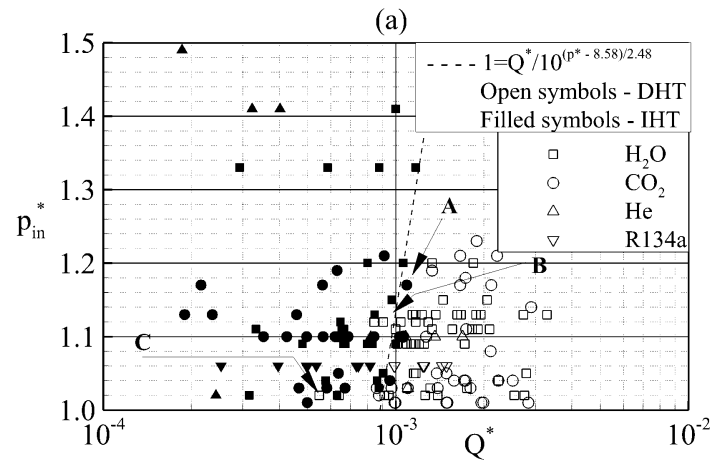

(c)

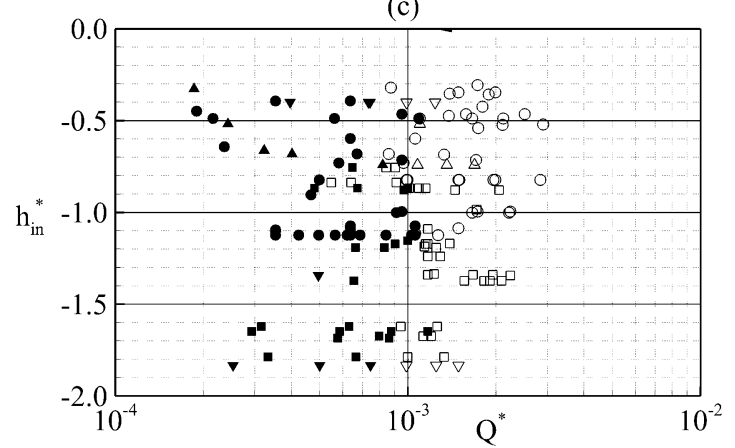

(e)

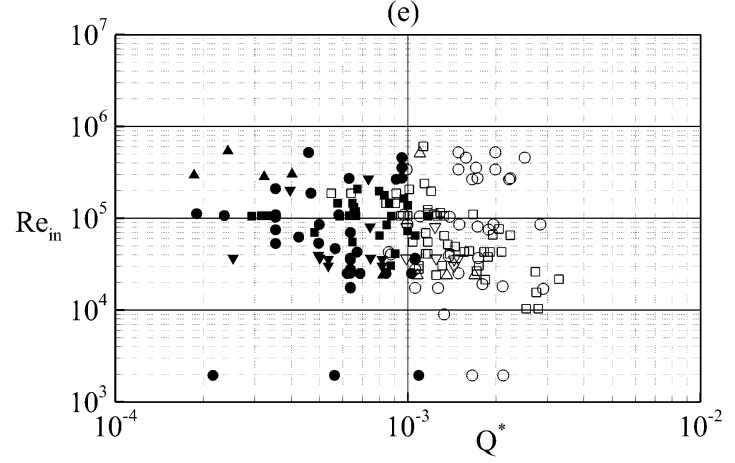

(b)

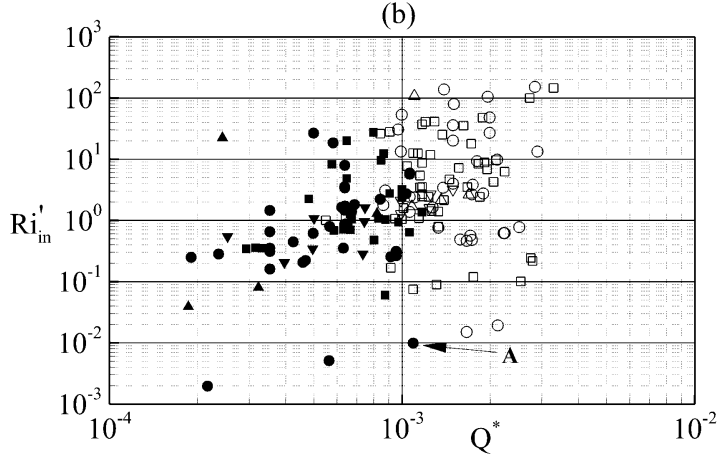

(d)

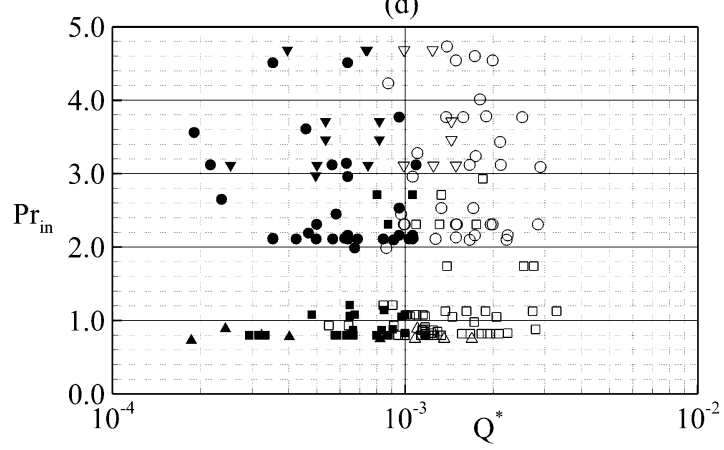

(f)

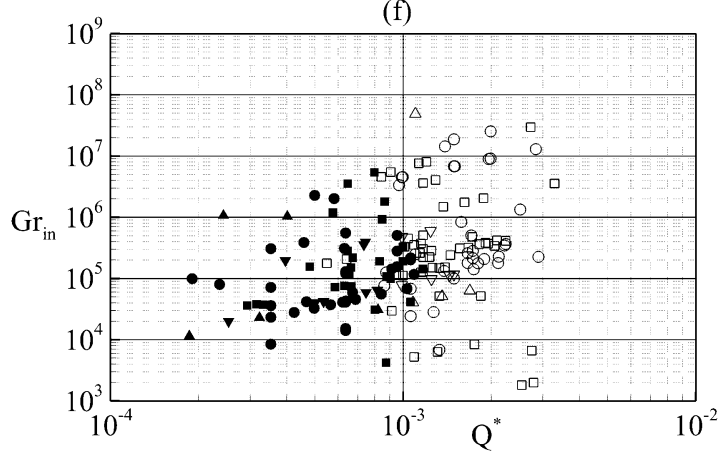

Figure 6-8: Published data assessed against pairs of similarity criteria for ducted flows with upstream-oriented gravitational acceleration.

$p^{*}, R i_{\text {in }}{ }^{\prime}$, and $Q^{*}$ as the dominant similarity criteria for establishing conditions conducive to IHT or DHT phenomena in ducted flows of downstream- and wall-normal-oriented gravitational acceleration, respectively. Although these figures indicate that the proposed similarity criteria favourably capture the DHT and IHT phenomena, the number of datasets available is insufficient to quantify a threshold value for these phenomena to an 

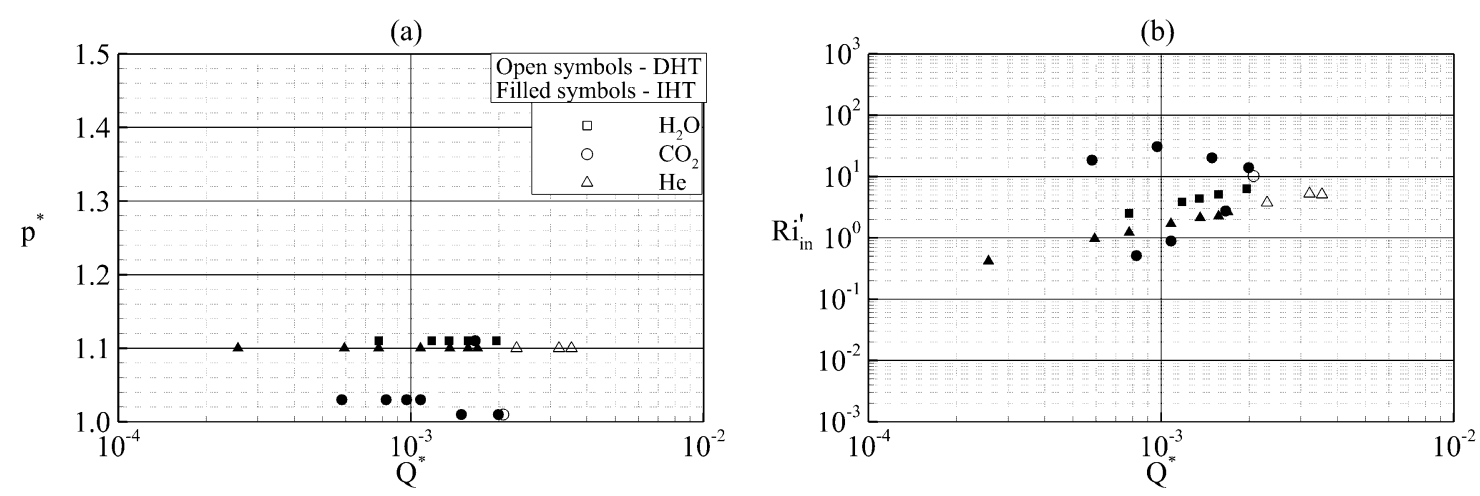

Figure 6-9: Published data assessed against pairs of $Q^{*} \& p^{*}$ and $Q^{*} \& R i_{\text {in }}^{\prime}$ for ducted flows with downstream-oriented gravitational acceleration.
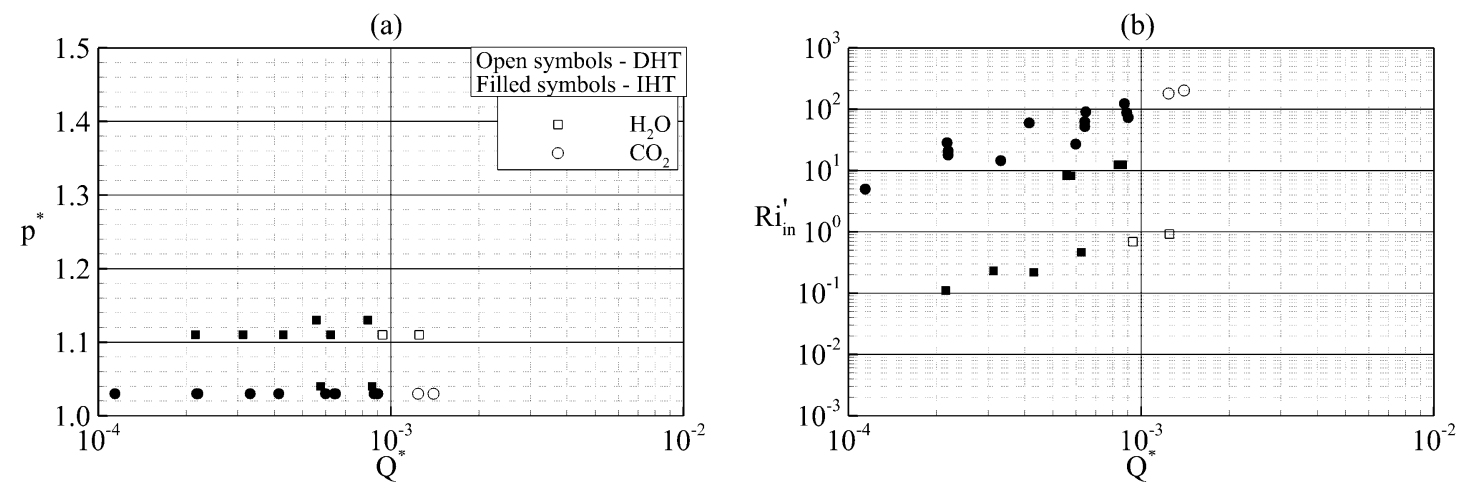

Figure 6-10: Published data assessed against pairs of $Q^{*} \& p^{*}$ and $Q^{*} \& R i_{\text {in }}^{\prime}$ for ducted flows with horizontal-oriented gravitational acceleration.

adequate degree of certainty. However, it can be deduced from Figure 6-9 that in flows with downstream-oriented gravitational acceleration, threshold values of $Q^{*}$ for IHT and DHT phenomena are about two times the threshold values with upstream-oriented gravitational acceleration at equivalent conditions. Conversely, the available data plotted in Figure 6-10 indicates that these threshold values are similar for wall-normal-oriented gravitational acceleration and upstream-oriented gravitational acceleration cases. 
Evaluating the adequacy of the proposed similarity criteria in matching dependent nondimensional parameters, such as Nusselt number $(\mathrm{Nu})$ distributions along the flow path, would require sets of data having values of the similarity criteria in Equation 6-32 that are consistent for multiple working fluids. Figure 6-11 (a) and (b) plot datasets depicting IHT phenomena for pairs of working fluids at particular values of $Q^{*}$ and $p^{*}$, and indicate favourable agreement in the trends of $N u$ variations with $h^{*}$. Notable deviation is observed at the downstream end of Song et al.'s (2008) $\mathrm{CO}_{2}$ data in Figure 6-11(b) compared to Yamagata et al.'s (1972) water data. The good agreement in $N u$ over a significant part of the upstream portion of these flows suggests that the observed discrepancy in the downstream portion may be due to flow effects induced by the proximity of the outflow boundary. Figure 6-11(c) and (d) plot datasets depicting DHT phenomena. The trends of $N u$ in Figure 6-11(c) are noted to be similar between Zhang et al.'s (2012) water data and Kim \& Kim's (2011) $\mathrm{CO}_{2}$ data. However, the sharp decrease in $N u$ downstream of $h^{*}=-0.5$ occurs less gradually for the $\mathrm{CO}_{2}$ data which is noted to have a lower value of $p^{*}$. Likewise, the less gradual variation of $N u$ with $h^{*}$ for data with a lower $p^{*}$ value is observed through comparison of Kang \& Chang's (2009) R134a data and Kirillov et al.'s (1986) (from Pioro \& Duffey, 2005) water data illustrated in Figure 6-11(d). These data depict similar upstream trends whereby a sharp decrease in $N u$ is observed beginning at $h^{*} \approx-1.0$, indicating the onset of DHT. However, the decrease is more rapid for the R134a data and the $N u$ magnitudes for this data are notably larger than those of the water data in the downstream region where the flow recovers from DHT. Both DHT and the recovery of the flow from DHT are governed by the effects of spatial thermophysical property gradients on local flow turbulence and hence convection heat 
transfer (Azih et al., 2012; Bae et al., 2008). Thus, the more rapid streamwise rates of change and larger magnitudes of $N u$ for the R134a data may be due to the relatively lower $p^{*}$ values for this data, where lower $p^{*}$ values are expected to yield larger spatial gradients in the thermophysical properties, as per Figure 6-1 to Figure 6-4, and thus yield more pronounced variations in $\mathrm{Nu}$.
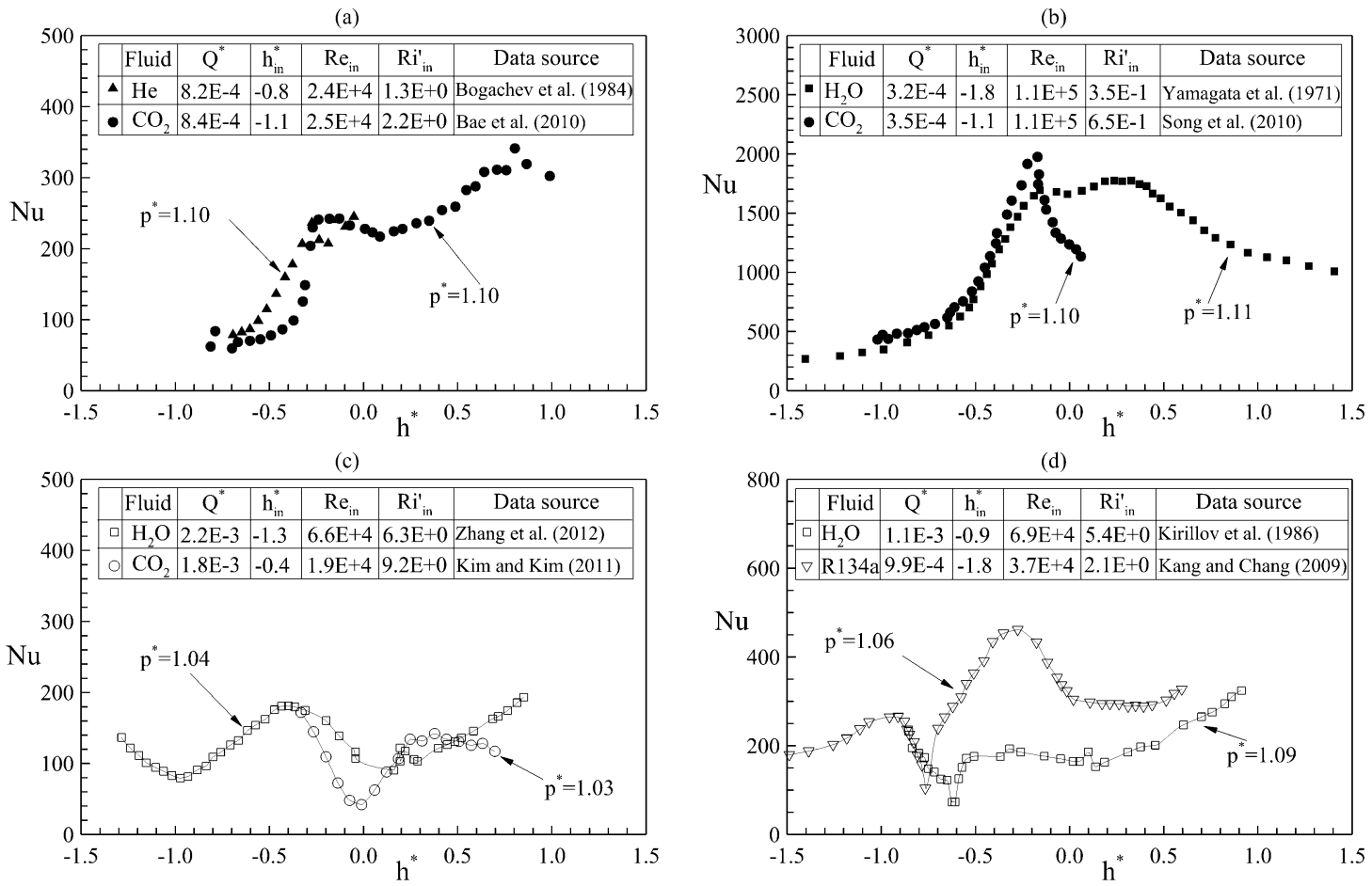

Figure 6-11: Streamwise variation of Nusselt number for pairs of working fluids at specific values of $Q^{*}$ and $p^{*}$ to yield: $(\mathrm{a}, \mathrm{b}) \mathrm{IHT} ;(\mathrm{c}, \mathrm{d})$ DHT.

The favourable categorization of IHT and DHT trends in Figure 6-7 to Figure 6-10, are promising signs for the adequacy of the proposed similarity criteria for mixed-convection heat transfer in supercritical fluids. Additional validation of these similarity criteria through investigation of streamwise variations of Nusselt number and quantification of 
threshold values for IHT and DHT phenomena would require targeted experiments to be conducted at flow states in the vicinity of the proposed threshold region between IHT and DHT. 


\section{Chapter: Conclusion and Recommendations}

\subsection{Conclusions}

DNS are used to identify the effects of the supercritical thermodynamic state on heat transfer in flows over flat surfaces and in channel flows. The simulations are performed in flow environments in the presence and absence of gravitational acceleration. In one instance the thermophysical properties are fixed to serve as a baseline for the remaining cases where these properties are permitted to vary. The cases that investigate buoyancy effects constitute environments with wall-normal gravitational acceleration and downstream-oriented gravitational acceleration. For the simulated conditions, it is observed that the inertia- and buoyancy-driven phenomena responsible for the unique characteristics of heat transfer in supercritical fluids are linked to the effect of the spatial gradients of the thermophysical properties on the development of hairpin vortices and their associated low- and high-speed streaks. Spatial gradients of density in particular are observed to play a primary role in the development of hairpin vortices through generation of vorticity via baroclinic mechanisms. In the absence of gravitational acceleration it is observed that baroclinic vorticity generation in the vicinity of inviscid-unstable shear layers cause these shear layers to more readily roll-up into hairpin vortices. These hairpin vortices are also significantly smaller in absolute streamwise and spanwise dimensions due to a reduction in the viscous length scales when the wall temperature is near the pseudo-critical temperature. These hairpin vortices destructively interact with hairpin vortices from the unheated upstream domain, yielding deterioration in coherence of both sets of hairpin vortices. Downstream of this region, low-speed streaks that are swept 
towards the wall undergo substantial streamwise acceleration due to volumetric expansion induced by heating from the wall. The subsequently generated unstable shear layers which spawn from this acceleration of near-wall fluid, contained wall-normal and spanwise vorticity that roll-up to form new hairpin vortices. Higher-temperature fluid is ejected away from the lower wall through induction between the legs of these hairpin vortices thereby improving thermal mixing in the wall-normal region where this motion dominates. In the case where the gravitational acceleration is oriented in the wall-normal direction, prevailing buoyancy force along the lower and upper channel walls causes generation of vorticity via the baroclinic mechanism with a sense of rotation that respectively promotes and suppresses the induced motions of the legs of near-wall hairpin vortices. The net effect is increased and reduced levels of thermal mixing along the upper and lower channel walls, respectively. In the case with downstream-oriented gravitational acceleration, generation of vorticity via the baroclinic mechanism driven by spanwise density gradients in the vicinity of the legs of existing hairpin vortices supplements the ejection of hotter fluid away from the wall. Likewise, the baroclinic instability mechanism driven by wall-normal density gradients in the vicinity of the heads of existing hairpin vortices supplements the sweep of colder fluid towards the wall. The net effect of these instabilities is to enhance wall-normal thermal mixing of the flow.

Based on the foregoing understanding of the physical phenomena in heat transfer to fluids at the supercritical thermodynamic state, a fluid-to-fluid model in the form of dynamic similarity parameters is established. Similarity criteria in the form of nondimensional parameters were derived to characterize mixed-convection heat transfer to 
ducted flows of supercritical fluids. The relevant dynamic similarity criteria and statespace similarity criteria were derived using the current physical understanding of heat transfer phenomena that lead to the unique heat transfer and flow characteristics of such flows. The state-space similarity criteria, consisting of a non-dimensional enthalpy $\left(h^{*}\right)$ and non-dimensional pressure $\left(p^{*}\right)$, were shown to outperform other state-space similarity criteria that have been proposed in published literature. The proposed set of similarity criteria with judicious choices of characteristic flow and fluid parameters comprised $h^{*}$, $p^{*}$, Reynolds number $(R e)$, and Richardson number $\left(R i^{\prime}\right)$ at the inflow, and the nondimensional heat transfer rate, $Q^{*}$, at the wall. This set of similarity criteria was validated using experimental data from published literature with four working fluids commonly used in heat transfer studies involving supercritical thermophysical states, namely: water; carbon dioxide; helium; and, R134a. The $Q^{*}$ and $p^{*}$ similarity criteria were demonstrated to respectively have primary and secondary influence on the development of improved and deteriorated heat transfer phenomena. Accordingly, based on these similarity criteria, threshold values are established that adequately predict fluid/flow conditions conducive to improved and deteriorated heat transfer phenomena with $\sim 95 \%$ accuracy for the analyzed flows with upstream-oriented gravitational acceleration. The proposed similarity criteria was shown to be a useful tool for analysis of Nusselt number trends for subsets of data having sufficiently similar values of these criteria. 


\subsection{Recommendations for Further Study}

- To further refine the proposed similarity criteria, DNS may be performed using different working fluids at equivalent conditions.

- Targeted experiments near the proposed threshold value for DHT, given by Equation 6-32, could be performed to further refine this threshold prediction.

- The current study investigates flows in wall-normal- and downstream- oriented gravitational acceleration. To complement these studies, a computation with upward-oriented gravitational acceleration at equivalent conditions to those of the simulations in Chapter 5 could be performed.

- In the simulations of the present study, the computational domains were not of sufficient length to capture the largest scales of turbulent motions. This meant that only a wall-normal portion of the flow field could be fully analysed and inlet conditions could not be chosen sufficiently far from the pseudo-critical point to mimic experiment setups. Computations with longer domains could be performed to better mimic experimental setups. 


\section{References}

Abe, H., Kawamura, H. \& Matsuo, Y., 2001, 'Direct numerical simulation of a fully developed turbulent channel flow with respect to the Reynolds number dependence', Transactions of the ASME, 123(2), pp.382-393.

Ackerman, J.W., 1970, 'Pseudoboiling heat transfer to supercritical pressure water in smooth and ribbed tubes', Journal of Heat Transfer, 92(2), pp.490-498.

Adebiyi, G.A. \& Hall, W.B., 1976, 'Experimental investigation of heat transfer to supercritical pressure carbon dioxide in a horizontal pipe', International Journal of Heat and Mass Transfer, 19(7), pp.715-720.

Adrian, R.J., 2007, 'Hairpin vortex organization in wall turbulence' Physics of Fluids, 19(4).

Adrian, R.J., Meinhart, C.D. \& Tomkins, C.D., 2000, 'Vortex organization in the outer region of the turbulent boundary layer', Journal of Fluid Mechanics, 422, pp.1-54.

Alekseev, G.V., Silin, V.A., Smirnov, A.M. \& Subbotin, V.I., 1976, 'Study of the thermal conditions on the wall of a pipe during the removal of heat by water at a supercritical pressure', High Temperature, 14(4), pp.683-687.

Ambrosini, W., 2011, 'Discussion of similarity principles for fluid-to-fluid scaling of heat transfer behaviour at supercritical pressures', Nuclear Engineering and Design, 241(12), pp.5149-5173.

Ambrosini, W., 2008, 'Discussion on the stability of heated channels with different fluids at supercritical pressures', Nuclear Engineering and Design, 239, pp. 2952-2963.

Andersson, P., Brandt, L., Bottaro, A. \& Henningson, D.S., 2001, 'On the breakdown of boundary layer streaks, Journal of Fluid Mechanics, 428, pp.29-60. 
ANSYS (2009), ANSYS CFX-solver theory guide, ANSYS, inc.

Azih, C. Brinkerhoff, J.R. \& Yaras, M.I., 2012, 'Direct numerical simulation of convective heat transfer in a zero-pressure-gradient boundary-layer with supercritical water', Journal of Thermal Science, 21(1), pp.49-59.

Bae, Y.Y., Kim, H.Y. \& Kang, D.J., 2010, 'Forced and mixed convection heat transfer to supercritical $\mathrm{CO}_{2}$ vertically flowing in a uniformly-heated circular tube', Experimental Thermal and Fluid Science, 34(8), pp.1295-1308.

Bae, J.H., Yoo, J.Y. \& McEligot, D.M., 2008, 'Direct numerical simulation of heated $\mathrm{CO} 2$ flows at supercritical pressure in a vertical annulus at $\mathrm{Re}=8900$ ', Physics of Fluids, 20(5), p.055108.

Bae, J.H., Yoo, J.Y. \& Choi, H., 2005, 'Direct numerical simulation of turbulent supercritical flows with heat transfer', Physics of Fluids, 17(10), p.105104.

Baig, M.F. \& Chernyshenko, S.I., 2004, 'Regeneration mechanism of streaks in near-wall quasi-2D turbulence', European Journal of Mechanics-B/Fluids, 23(5), pp.727736.

Bazargan, M. \& Mohseni, M., 2012, 'Algebraic zero-equation versus complex twoequation turbulence modeling in supercritical fluid flows', Computers \& Fluids, 60, pp.49-57.

Bernard, P.S., Thomas, J.M. \& Handler, R.A., 1993, 'Vortex dynamics and the production of Reynolds stress', Journal of Fluid Mechanics, 253, pp.385-419.

Bishop, A.A., Sandberg, L.O. \& Tong, L.S., 1964, 'Forced convection heat transfer to water at near critical temperatures and supercitical pressures', A.I.Ch.E.-I.Chem.E Symposium Series No. 2, 85. 
Bogachev, V.A., Yeroshenko, V.M. \& Yaskin, L.A., 1984, 'Measurements of mixed convection heat transfer to upflow of supercritical helium in a vertical tube', Cryogenics, 24(5), pp.266-270.

Bourke, P.J., Pulling, D.J., Gill, L.E. \& Denton, W.H., 1970, 'Forced convective heat transfer to turbulent $\mathrm{CO}_{2}$ in the supercritical region', International Journal of Heat and Mass Transfer, 13(8), pp.1339-1348.

Brassington, D.J. \& Cairns, D.N.H., 1977, 'Measurements of forced convective heat transfer to supercritical helium', International Journal of Heat and Mass Transfer, 20(3), pp.207-214.

Brinkerhoff, J.R. \& Yaras, M.I., 2015, 'Numerical investigation of transition in a boundary layer subjected to favourable and adverse streamwise pressure gradients and elevated free stream turbulence', Journal of Fluid Mechanics, 781, pp.52-86.

Brinkerhoff, J.R. \& Yaras, M.I., 2014, 'Numerical investigation of the generation and growth of coherent flow structures in a triggered turbulent spot', Journal of Fluid Mechanics, 759, pp.257-294.

Brinkerhoff, J.R., 2014, Numerical Investigations of Instability and Transition in Attached and Separated Shear Layers, Ph. D. Thesis, the Carleton University, Ottawa, Canada.

Buschmann, M.H., Indinger, T. \& Gad-el-Hak, M., 2009, 'Near-wall behavior of turbulent wall-bounded flows', International Journal of Heat and Fluid Flow, 30(5), pp.993-1006. 
Butler, K.M. \& Farrell, B.F., 1993, 'Optimal perturbations and streak spacing in wall-bounded turbulent shear flow', Physics of Fluids A: Fluid Dynamics, 5(3), pp.774-777.

Camussi, R. \& Di Felice, F., 2006, 'Statistical properties of vortical structures with spanwise vorticity in zero pressure gradient turbulent boundary layers', Physics of Fluids, 18(3), p.035108.

Cess, R. D. \& Shaffer. E. C., 1959, 'Heat transfer to laminar flow between parallel plates with a prescribed wall heat flux'. Applied Scientific Research, Section A 8.1, pp.339-344.

Chatoorgoon, V., 2013, 'Non-dimensional parameters for static instability in supercritical heated channels', International Journal of Heat and Mass Transfer, 64, pp.145-154

Chatoorgoon, V., 2008, 'Supercritical flow stability in horizontal channels', Nuclear Engineering and Design, 238, pp. 1940-1946.

Cheng, X., Liu, X.J. \& Gu, H.Y., 2011, "Fluid-to-fluid" scaling of heat transfer in circular tubes cooled with supercritical fluids', Nuclear Engineering and Design, 241(2), pp.498-508.

Cheng, X., Kuang, B. \& Yang, Y.H., 2007, 'Numerical analysis of heat transfer in supercritical water cooled flow channels', Nuclear Engineering and Design, 237(3), pp.240-252.

Cheng, X. \& Schulenberg, T., 2001, 'Heat transfer at supercritical pressures: literature review and application to an HPLWR', Technik und Umwelt, Wissenschaftliche Berichte, Institute für Kernund Energietechnik, FZKA 6609. 
Chin, C., Ooi, A.S.H., Marusic, I. \& Blackburn, H.M., 2010, 'The influence of pipe length on turbulence statistics computed from direct numerical simulation data', Physics of Fluids, 22(11), p.115107.

Churchill, S.W., 1976, 'A comprehensive correlating equation for forced convection from flat plates', AIChE Journal, 22(2), pp.264-268.

Colebrook, C.F. \& White, C.M., 1937, 'Experiments with fluid friction in roughened pipes', Proceedings of the Royal Society of London. Series a, Mathematical and Physical Sciences, pp.367-381.

Cooley, J.W. \& Tukey, W., 'An algorithm for the machine computation of complex Fourier series', Mathematics of Computation, 19(29), pp.7-30.

Corino, E.R., \& Brodkey, R.S., 1969, 'A visual investigation of the wall region in turbulent flow', Journal of Fluid Mechanics, 37, pp.1-30.

Cramer, M.S. \& Bahmani, F., 2014, 'Effect of large bulk viscosity on large-Reynoldsnumber flows', Journal of Fluid Mechanics, 751, pp.142-163.

Dave, N., Azih, C. \& Yaras, M.I., 2013, 'A DNS study on the effects of convex streamwise curvature on coherent structures in a temporally-developing turbulent boundary layer with supercritical water', International Journal of Heat and Fluid Flow, 44, pp.635-643.

De Rosa, M., Guetta, G., Ambrosini, W., Forgione, N., He, S. \& Jackson, J.D., 2011, 'Lessons learned from the application of CFD models in the prediction of heat transfer to fluids at supercritical pressure', The 5th Int. Sym. SCWR (ISSCWR-5) P, 60, pp.13-16. 
Dubief, Y. \& Delcayre, F., 2000, 'On coherent-vortex identification in turbulence', Journal of Turbulence, 1(1), pp.011-011.

Duffey, R.B. \& Pioro, I.L., 2005, 'Experimental heat transfer of supercritical carbon dioxide flowing inside channels (survey)', Nuclear Engineering and Design, 235(8), pp.913-924.

Eckelmann, H., 1974, 'The structure of the viscous sub layer and the adjacent wall region in a turbulent channel flow', Journal of Fluid Mechanics, 65, pp.439-459.

Elsinga, G.E. \& Marusic, I., 2010, 'Evolution and lifetimes of flow topology in a turbulent boundary layer', Physics of Fluids, 22(1), p.015102.

Fathali, M., Klein, M., Broeckhoven, T., Lacor, C. \& Baelmans, M., 2008, 'Generation of turbulent inflow and initial conditions based on multi-correlated random fields', International Journal for Numerical Methods in Fluids, 57(1), pp.93-117.

Favre, A.J., (1965), The Equations of Compressible Turbulent Gases, Aix-Marsille Univeriste (France) Inst de Mechanique Stastisque de la Turbulence, Report AD622097.

Fewster, J., 1976, Mixed forced and free convective heat transfer to supercritical pressure fluids flowing in vertical pipes, Ph.D. thesis, the University of Manchester, UK.

Friedrich, R., Hüttl, T.J., Manhart, M. \& Wagner, C., 2001, 'Direct numerical simulation of incompressible turbulent flows', Computers \& Fluids, 30(5), pp.555-579.

Giarratano, P.J. \& Jones, M.C., 1975, 'Deterioration of heat transfer to supercritical helium at 2 - 5 atmospheres', International Journal of Heat and Mass Transfer, 18(5), pp.649-653. 
Giarratano, P.J., Arp, V.D. \& Smith, R.V., 1971, 'Forced convection heat transfer to supercritical helium', Cryogenics, 11(5), pp.385-393.

GIF, 2009, GIF R\&D Outlook for generation IV nuclear systems. Available from: $<$ http://www.gen-4.org/PDFs/GIF_RD_Outlook_for_Generation_IV_Nuclear_ Energy_Systems.pdf $>$. (3 May 2011)

Gupta, S., Saltanov, E., Mokry, S.J., Pioro, I., Trevani, L. \& McGillivray, D., 2013, 'Developing empirical heat-transfer correlations for supercritical $\mathrm{CO}_{2}$ flowing in vertical bare tubes', Nuclear Engineering and Design, 261, pp.116-131.

Hall, W.B. \& Jackson, J.D., 1969, 'Laminarization of a turbulent pipe flow by buoyancy forces', ASME paper. no 69-HT-55.

Hall, W.B., Jackson, J.D. \& Watson, A., 1967, September. 'Paper 3: A Review of Forced Convection Heat Transfer to Fluids at Supercritical Pressures', In Proceedings of the Institution of Mechanical Engineers, Conference Proceedings Vol. 182, No. 9, pp. 10-22, SAGE Publications.

Hasan, N. \& Farouk, B., 2012, 'Thermoacoustic transport in supercritical fluids at nearcritical and near-pseudo-critical states', The Journal of Supercritical Fluids, 68, pp.13-24.

He ${ }^{\mathrm{a}}$, S., Kim, W.S. \& Bae, J.H., 2008,' Assessment of performance of turbulence models in predicting supercritical pressure heat transfer in a vertical tube', International Journal of Heat and Mass Transfer, 51(19), pp.4659-4675.

$\mathrm{He}^{\mathrm{b}}$, S., Kim, W.S. \& Jackson, J.D., 2008, 'A computational study of convective heat transfer to carbon dioxide at a pressure just above the critical value', Applied Thermal Engineering, 28(13), pp.1662-1675. 
He, S., Jiang, P.X., Xu, Y.J., Shi, R.F., Kim, W.S. \& Jackson, J.D., 2005, A computational study of convection heat transfer to $\mathrm{CO}_{2}$ at supercritical pressures in a vertical mini tube, International Journal of Thermal Sciences, 44(6), pp.521-530.

Head, M.R. \& Bandyopadhyay, P., 1981, 'New aspects of turbulent boundary-layer structure', Journal of Fluid Mechanics, 107, pp.297-338.

Hofmeister, J., Waata, C., Starflinger, J., Schulenberg, T. \& Laurien, E., 2007, 'Fuel assembly design study for a reactor with supercritical water' Nuclear Engineering and Design, 237(14), pp.1513-1521.

Holman, J.P., 2002. Heat Transfer, 9th ed., McGraw-Hill, New York.

Hoyas, S. \& Jimenez, J., 2006, 'Scaling of the velocity fluctuations in turbulent channels up to $\mathrm{Re}_{\tau}=2003$ ', Physics of Fluids. 18(1), p.011702.

Hutchins, N. \& Marusic, I., 2007, 'Evidence of very long meandering features in the logarithmic region of turbulent boundary layers', Journal of Fluid Mechanics, 579, pp.1-28.

Hutchinson, B.R. \& Raithby, G.D., 1986, 'A multigrid method based on the additive correction strategy', Numerical Heat Transfer, Part A: Applications, 9(5), pp.511537.

IAPWS, 2009, Reviced release on the IAPWS formulation 1995 for the thermodynamic properties of ordinary water substance for general scientific use, The International Association for the Properties of Water and Steam.

IAPWS, 2008, 'Release on the IAPWS formulation 2008 for the viscosity of ordinary water substance' In: The International Association for the Properties of Water and Steam. Berlin, Germany: pp.1-9. 
Jackson, J.D., 2011, 'A model of developing mixed convection heat transfer in vertical tubes to fluids at supercritical pressure' International Symposium on Supercritical Water-Cooled Reactors, Vancouver, British Columbia, Canada, P104.

Jackson, J.D. Jiang, P.X. Liu, B. \& Zhao, C.R., 2011, 'Interpreting and categorizing experimental data on forced and mixed convective heat transfer to supercritical pressure fluids using physically-based models', Proceedings of the 5th International Symposium on Supercritical Water-Cooled Reactors, Vancouver, British Columbia, Canada. P103.

Jackson, J.D. \& Hall, W.B., 1979, 'Influences of buoyancy on heat transfer to fluids flowing in vertical tubes under turbulent conditions', Turbulent forced convection in channels and bundles, 2, pp.613-640.

Jaromin, M. \& Anglart, H., 2013, 'A numerical study of heat transfer to supercritical water flowing upward in vertical tubes under normal and deteriorated conditions', Nuclear Engineering and Design, 264, pp.61-70.

Jiang, P.X., Zhang, Y., Zhao, C.R. \& Shi, R.F., 2008, 'Convection heat transfer of $\mathrm{CO}_{2}$ at supercritical pressures in a vertical mini tube at relatively low Reynolds numbers', Experimental Thermal and Fluid Science, 32(8), pp.1628-1637.

Jiang, X. \& Luo, K.H., 2000, 'Direct numerical simulation of the puffing phenomenon of an axisymmetric thermal plume', Theoretical and Computational Fluid Dynamics, 14(1), pp.55-74.

Jimenez, J. \& Pinelli, A., 1999, 'The autonomous cycle of near-wall turbulence', Journal of Fluid Mechanics, 389, 335. 
Jimenez, J. \& Moin, P., 1991, 'Minimal flow unit in near wall turbulence', Journal of Fluid Mechanics, 225, pp.213-140.

Kachanov, Y.S., 1994, 'Physical mechanisms of laminar-boundary-layer transition', Annual Review of Fluid Mechanics, 26(1), pp.411-482.

Kader, B.A., 1981, 'Temperature and concentration profiles in fully turbulent boundary layers', International Journal of Heat and Mass Transfer, 24(9), pp.1541-1544.

Kang, K.H. \& Chang, S.H., 2009, 'Experimental study on the heat transfer characteristics during the pressure transients under supercritical pressures', International Journal of Heat and Mass Transfer, 52(21), pp.4946-4955.

Kao, M.T., Lee, M. Ferng, Y. M. \& Chieng, C.C., 2010, 'Heat transfer deterioration in a supercritical water channel', Nuclear Engineering and Design, 240, pp.3321-3328.

Kasagi, N. \& Shikazono, N., 1995, October, 'Contribution of direct numerical simulation to understanding and modelling turbulent transport', In Proceedings of the Royal Society of London A: Mathematical, Physical and Engineering Sciences, Vol. 451, No. 1941, pp. 257-292.

Kawamura, H., Ohsaka, K., Abe, H. \& Yamamoto, K., 1998, 'DNS of turbulent heat transfer in channel flow with low to medium-high Prandtl number fluid', International Journal of Heat and Fluid Flow, 19(5), pp.482-491.

Khartabil, H. \& Leung, L., 2009, Spetember, 'SCWR overview', in GIF Symposium. Paris, France.

Kim, D.E. \& Kim, M.H., 2011, 'Experimental investigation of heat transfer in vertical upward and downward supercritical $\mathrm{CO} 2$ flow in a circular tube', International Journal of Heat and Fluid Flow, 32, pp.176-191. 
Kim, J., Moin, P., \& Moser R.D., 1987, 'Turbulence statistics in fully developed channel flow and low Reynolds number', Journal of Fluid Mechanics, 177, pp.133-166.

Klebanoff, P.S. \& Tidstrom, K.D., 1959, 'Evolution of amplified waves leading to transition in a boundary layer with zero pressure gradient', National Aeronautics and Space Administration, 195.

Kolmogorov, A.N., 1991, 'Local structure of turbulence in incompressible viscous fluid for large Reynolds number', Proceedings of the Royal Society A. 434, pp.9-13.

Kong, H., Choi, H. \& Lee, J.S., 2000, 'Direct numerical simulation of turbulent thermal boundary layers', Physics of Fluids, 12(10), pp.2555-2568.

Koshizuka, S., Takano, N. \& Oka, Y., 1995, 'Numerical analysis of deterioration phenomena in heat transfer to supercritical water', International Journal of Heat and Mass Transfer, 38(16), pp.3077-3084.

Krau, C.I., Kuhn, D., \& Schulenberg, T., 2008., 'Heat transfer phenomena of supercritical fluids', International Youth Nuclear Conference, Inkerlaken, Switzerland, P352.

Kurganoc, V.A. \& Kaptilnyi, A.G., 1993, 'Flow structure and turbulent transport of supercritical pressure fluid in a vertical heated tube under the conditions of mixed convection. Experimental data', International Journal of Heat and Mass Transfer, 36(13), pp.3383-3392.

Lei, X., Li, H., Yu, S. \& Chen, T., 2012, 'Numerical investigation on the mixed convection and heat transfer of supercritical water in horizontal tubes in the large specific heat region', Computers \& Fluids, 64, pp.127-140.

Lele, S.K., 1994, 'Compressibility effects on turbulence', Annual Review of Fluid Mechanics, 26(1), pp.211-254. 
Lemmon, E.W., McLinden M.O., \& Friend, D.G., 'Thermophysical Properties of Fluid Systems, in NIST Chemistry WebBook', NIST Standard Reference Database Number 69, Eds. Linstrom P.J. and. Mallard W.G, National Institute of Standards and Technology, Gaithersburg MD, 20899, http://webbook.nist.gov, (retrieved January 2011).

Lesieur, M., Metais, O., \& Comte, P., 2005, Large Eddy Simulation of Turbulence, Cambridge University Press, Cambridge.

Li, H., Kruizenga, A., Anderson, M., Corradini, M., Luo, Y., Wang, H. \& Li, H., 2011, 'Development of a new forced convection heat transfer correlation for CO 2 in both heating and cooling modes at supercritical pressures', International Journal of Thermal Sciences, 50(12), pp.2430-2442.

Li, Z.H., Jiang, P.X., Zhao, C.R. \& Zhang, Y., 2010, 'Experimental investigation of convection heat transfer of $\mathrm{CO}_{2}$ at supercritical pressures in a vertical circular tube', Experimental Thermal and Fluid Science, 34(8), pp.1162-1171.

Li, X., Hashimoto, K., Tominaga, Y., Tanahashi, M. \& Miyauchi, T., 2007, 'Numerical study of heat transfer mechanism in turbulent supercritical $\mathrm{CO}_{2}$ channel flow', Journal Thermal Science and Technology, 3, pp.112-123.

Marusic, I., McKeon, B.J., Monkewitz, P.A., Nagib, H.M., Smits, A.J. \& Sreenivasan, K.R., 2010, 'Wall-bounded turbulent flows at high Reynolds numbers: Recent advances and key issues', Physics of Fluids, 22(6), pp.065103.

McAuliffe, B.R. \& Yaras, M.I., 2008, 'Numerical study of turbulent-spot development in a separated shear layer', Journal of Turbomachinery, 130(4), pp.041018. 
McEligot, D.M. \& Jackson J.D., 2004, “"Deterioration” criteria for convective heat transfer in gas flow through non-circular ducts', Nuclear Engineering and Design, 232, pp.327-333.

Mellor, G.L. \& Herring, H.J., 1973, 'A survey of the mean turbulent field closure models', American Institute of Aeronautics and Astronautics Journal, 11(5), pp.590-599.

Metaias, B. \& Eckert, E.R.G., 1964, 'Forced, mixed, and free convection regimes', Journal of Heat Transfer, 86(2), pp.295-296.

Mohseni, M. \& Bazargan, M., 2012., 'Modification of low Reynolds number k- $\varepsilon$ turbulence models for applications in supercritical fluid flows', International Journal of Thermal Science, 51, pp.51-62.

Moin, P. \& Mahesh, K., 1998, 'Direct numerical simulation: A tool in turbulent research', Annual Review of Fluid Mechanics, 30, pp.539-578.

Mokry, S., Pioro, I., Farah, A., King, K., Gupta, S., Peiman, W. \& Kirillov, P., 2011, 'Development of supercritical water heat-transfer correlation for vertical bare tubes', Nuclear Engineering and Design, 241(4), pp.1126-1136.

Monty, J.P. \& Chong, M.S., 2009, 'Turbulent channel flow: comparison of streamwise velocity data from experiments and direct numerical simulation', Journal of Fluid Mechanics, 633, pp.461-474.

Nallasamy, M., 1987, 'Turbulence models and their applications to the prediction of internal flows: a review', Computers \& Fluids, 15(2), pp.151-194. 
Nemati, H., Patel, A., Boersma, B.J., \& Pecnik, R., 2016, 'The effect of thermal boundary conditions on forced convection heat transfer to fluids at supercritical pressure', Journal of Fluid Mechanics, 800,pp.531-556.

Nemati, H., Patel, A., Boersma, B.J., \& Pecnik, R., 2015, 'Mean statistics of a heated turbulent pipe flow at supercritical pressure', International Journal of Heat and Mass Transfer, 83, pp.741-752.

Ničeno, B. \& Sharabi, M., 2013, 'Large eddy simulation of turbulent heat transfer at supercritical pressures', Nuclear Engineering and Design, 261, pp.44-55.

Österlund, J.M., Lindgren, B. \& Johansson, A.V., 2003, 'Flow structures in zero pressure-gradient turbulent boundary layers at high Reynolds numbers', European Journal of Mechanics-B/Fluids, 22(4), pp.379-390.

Özgen, S., 2004, 'Effect of heat transfer on stability and transition characteristics of boundary-layers'. International Journal of Heat and Mass Transfer, 47(22), pp.4697-4712.

Panton, R.L., 2001, 'Overview of the self-sustaining mechanisms of wall turbulence', Progress in Aerospace Sciences, 37(4), pp.341-383.

Peeters J.R.W., Pecnik, R., Rohde, M., van der Hagen, T.H.J.J., \& Boersma B.J., 2016, 'Turbulence attenuation in simultaneously heated and cooled annular flows at supercritical pressure', Journal of Fluid Mechanics, 799, pp.505-540.

Petukhov, B.S., Kurganov, V.A. \& Ankudinov, V.B., 1983, 'Heat transfer and flow resistance in the turbulent pipe flow of a fluid with near-critical state parameters', High Temperature Science, 21, pp.81-89. 
Pioro, I.L. \& Duffey, R.B., 2005, 'Experimental heat transfer in supercritical water flowing inside channels (survey)', Nuclear Engineering and Design, 235(22), pp.2407-2430.

Pioro', I.L., Duffey, R.B. \& Dumouchel, T.J., 2004, 'Hydraulic resistance of fluids flowing in channels at supercritical pressures (survey)', Nuclear Engineering and Design, 231(2), pp.187-197.

Pioro $^{\text {b }, ~ I . L ., ~ K h a r t a b i l, ~ H . F . ~ \& ~ D u f f e y, ~ R . B ., ~ 2004, ~ ' H e a t ~ t r a n s f e r ~ t o ~ s u p e r c r i t i c a l ~ f l u i d s ~}$ flowing in channels-empirical correlations (survey)', Nuclear Engineering and Design, 230(1), pp.69-91.

Pis'menny, E.N., E.N., Razumovskiy, V.G., Maevskiy, E.M., Koloskov, A.E., Pioro, I.L., 2006, 'Heat transfer to supercritical water in gaseous state or affected by mixed convection in vertical tubes', Proceedings of $14^{\text {th }}$ International Conference on Nuclear Engineering, ICONE14-89483, July 17-20, Miami, USA.

Pope, S.B., 2000, Turbulent Flows, Cambridge: Cambridge University Press.

Raw, M., 1996, 'Robustness of coupled algebraic multigrid for the Navier-Stokes equations', AIAA paper, 96, pp.0297.

Rayleigh, L., 1879, 'On the Stability, or Instability, of certain Fluid Motions', Proceedings of the London Mathematical Society, 1(1), pp.57-72.

Reinink, S.K. \& Yaras, M.I., 2015, 'Study of coherent structures of turbulence with large wall-normal gradients in thermophysical properties using direct numerical simulation', Physics of Fluids, 27(6), p.065113.

Robinson, S.K., 1991, 'Coherent motions in the turbulent boundary layer', Annual Review of Fluid Mechanics, 23(1), pp.601-639. 
Rodi, W., 1980, Turbulence Models and Their Application in Hydraulics - A State-oftheArt Review, IA International Association of Hydraulic Research Publication, Delft, The Netherlands.

Rohsenow, W.M., Hartnett, J.P. \& Cho, Y.I., 1998, Handbook of Heat Transfer. 3 ed., McGraw-Hill.

Schäfer, P., Severin, J. \& Herwig, H., 1995, 'The effect of heat transfer on the stability of laminar boundary layers', International Journal of Heat and Mass Transfer, 38(10), pp.1855-1863.

Schlatter, P., Orlu, R., Li, Q., Brethouwer, G., Fransson, J.H., Johansson, A.V., Alfredsson, P.H. \& Henningson, D.S., 2009, 'Turbulent boundary layers up to $\operatorname{Re} \theta=2500$ studied through simulation and experiment', Physics of Fluids, 21(5), p.51702.

Schlichting, H. \& Gersten, K., 2000, Boundary Layer Theory, 8th ed. Germany: SpringerVerlag Berlin Heidelberg.

Schröder, A. Geisler, R. Elsinga, G.E. Scarano, F. \& Dierksheide, U., 2008, 'Investigation of a turbulent spot and a tripped turbulent boundary layer flow using time resolved topographic PIV', Experiments in Fluids, 44, pp.305-316.

Schröder, A. \& Kompenhans, J., 2004, 'Investigation of a turbulent spot using multiplane stereo particle image velocimetry', Experiments in Fluids, 36(1), pp.82-90.

Shiralkar, B.S., \& Griffith, P., 1968, 'The deterioration in heat transfer to fluids at supercritical pressure and high heat fluxes', Dept. Mech. Eng. Massachusetts Institute of Technology, Report No. DSR 70332-55. 
Shitsman, M. W., 1974, 'Heat transfer to supercritical helium, carbon dioxide, and water: analysis of thermodynamic and transport properties and experimental data', Cryogenics, 14(2), pp.77-83.

Shitsman, M.E., 1963, 'Impairment of the heat transmission at supercritical pressures', Teplofiz Vysokikh Temperatur, 1(2), pp. 267-275.

Singer, B.A. \& Joslin, R.D., 1994, 'Metamorphosis of a hairpin vortex into a young turbulent spot', Physics of Fluids, 6(11), pp.3724-3736.

Skote, M., Haritonidis, J.H. \& Henningson, D.S., 2002, 'Varicose instabilities in turbulent boundary layers', Physics of Fluids, 14(7), pp.2309-2323.

Sleicher, C.A. \& Rouse, M.W., 1975, 'A convenient correlation for heat transfer to constant and variable property fluids in turbulent pipe flow', International Journal of Heat and Mass Transfer, 18(5), pp.677-683.

Smith, C.R. \& Metzler, S.P., 1983, 'The characteristics of low-speed streaks in the nearwall region of a turbulent boundary layer', Journal of Fluid Mechanics, 129, pp.2754.

Smits, A.J. \& Delo, C., 2001, 'Self-sustaining mechanisms of wall turbulence'. In Coherent Structures in Complex Systems, Springer Berlin Heidelberg, pp. 17-38.

Sobhan, C.B. \& Garimella, S.V., 2001, 'A comparative analysis of studies on heat transfer and fluid flow in microchannels', Microscale Thermophysical Engineering, 5(4), pp.293-311.

Song, J.H., Kim, H.Y., Kim, H. \& Bae, Y.Y., 2008, 'Heat transfer characteristics of a supercritical fluid flow in a vertical pipe', The Journal of Supercritical Fluids, 44(2), pp.164-171. 
Spalart, P.R., 1988, 'Direct simulation of a turbulent boundary layer up to $\operatorname{Re} \theta=1410$ ', Journal of Fluid mechanics, 187, pp.61-98.

Sreenivas, K.R. \& Prasad, A.K., 2000, 'Vortex-dynamics model for entrainment in jets and plumes', Physics of Fluids, 12(8), pp.2101-2107.

Stanislas, M., Perret, L. \& Foucaut, J.M., 2008, 'Vortical structures in the turbulent boundary layer: a possible route to a universal representation', Journal of Fluid Mechanics, 602, pp.327-382.

Styrikovich, M.A., Margulova, T.K. \& Miropol'Skii, Z.L., 1967, 'Problems in the development of designs of supercritical boilers', Teploenergetika, 14(6), pp.4-7.

Swenson, H.S., Carver, J.R. \& Kakarala, C.D., 1965, 'Heat transfer to supercritical water in smooth-bore tubes', Journal of Heat Transfer, 87(4), pp.477-483.

Tanahashi, M., Kang, S.J., Miyamoto, T., Shiokawa, S. \& Miyauchi, T., 2004, 'Scaling law of fine scale eddies in turbulent channel flows up to $\mathrm{Re}_{\tau}=800$ ', International Journal of Heat and Fluid Flow, 25(3), pp.331-340.

Tanehill, J.C., Anderson, DA. \& Pletcher, R.H., 1997, Computational Fluid Mechanics and Heat Transfer, 2 ed. Philadelphia, Taylor\&Francis.

Tomkins, C.D. \& Adrian, R.J., 2003, 'Spanwise structure and scale growth in turbulent boundary layers', Journal of Fluid Mechanics, 490, pp.37-74.

Van der Kraan, M., Peeters, M.M.W., Cid, M.F., Woerlee, G.F., Veugelers, W.J.T. \& Witkamp, G.J., 2005, 'The influence of variable physical properties and buoyancy on heat exchanger design for near-and supercritical conditions', The Journal of Supercritical Fluids, 34(1), pp.99-105. 
Vikhrev, Y.V., Barulin, Y.D. \& Kon'Kov, A.S., 1967, 'A study of heat transfer in vertical tubes at supercritical pressures', Thermal Engineering, 14(9), pp.116-119.

Wagner, W. \& Kruse A., 1997, 'The industrial standard IAWPS-IF97 for the thermodynamic properties and supplementary equations for other properties, Properties of water and steam'. Heidelberg:Springer Verlag.

Wagner, C., Hüttl, T.J. \& Friedrich, R., 2001, 'Low-Reynolds-number effects derived from direct numerical simulations of turbulent pipe flow', Computers \& Fluids, 30(5), pp.581-590.

Watts, M.J. \& Chou, C.T., 1982, September, 'Mixed convection heat transfer to supercritical pressure water', In Proceedings of the 7th International Heat Transfer Conference, 3, pp. 495-500.

Wazzan, A.R., Keltner, G., Okamura, T.T. \& Smith, A.M.O., 1972, 'Spatial stability of stagnation water boundary layer with heat transfer', Physics of Fluids, 15(12), pp.2114-2118.

White, F., 1991. Viscous Fluid Flow. New York: McGraw-Hill.

Wilcox, D., 2006. Turbulence Modelling for CFD, 3 ed. DCW industries.

Winterton, R.H.S., 1998, 'Where did the Dittus and Boelter equation come from?' International Journal of Heat and Mass Transfer, 41, pp.809-810.

Wu, X. \& Moin, P., 2010, 'Transitional and turbulent boundary layer with heat transfer', Physics of Fluids, 22(8), p.085105.

Wu, X. \& Moin, P., 2009, 'Direct numerical simulation of turbulence in a nominally zero-pressure-gradient flat-plate boundary layer', Journal of Fluid Mechanics, 630, pp.5-41. 
Yamagata, K., Nishikawa, K., Hasegawa, S., Fujii, T. \& Yoshida, S., 1972, 'Forced convective heat transfer to supercritical water flowing in tubes', International Journal of Heat and Mass Transfer, 15(12), pp.2575-2593.

Yoo, J.Y., 2013. 'The Turbulent Flows of Supercritical Fluids with Heat Transfer' Annual Review of Fluid Mechanics, 45, pp.494-525.

Yu, S., Li, H., Lei, X., Feng, Y., Zhang, Y., He, H. \& Wang, T., 2013, 'Experimental investigation on heat transfer characteristics of supercritical pressure water in a horizontal tube', Experimental Thermal and Fluid Science, 50, pp.213-221.

Zahlan, H., Groeneveld, D., and Tavoularis, S., 2015. 'Measurements of convective heat transfer to vertical upward flows of $\mathrm{CO}_{2}$ in circular tubes at near-critical and supercritical pressures'. Nuclear Engineering and Design, 289, pp.92-107.

Zahlan, H., Groeneveld, D.C., \& Tavoularis, S., 2014, 'Fluid-to-fluid scaling for convective heat transfer in tubes at supercritical and high subcritical pressures', International Journal of Heat and Mass Transfer, 73, pp.274-283.

Zhang, S., Gu, H., Cheng, X. \& Xiong, Z., 2014, 'Experimental study on heat transfer of supercritical Freon flowing upward in a circular tube', Nuclear Engineering and Design, 280, pp.305-315.

Zhang, G., Zhang, H., Gu, H., Yang, Y. \& Cheng, X., 2012, 'Experimental and numerical investigation of turbulent convective heat transfer deterioration of supercritical water in vertical tube', Nuclear Engineering and Design, 248, pp.226-237.

Zhang, Y., Zhang, C. \& Jiang, J., 2011, 'Numerical simulation of heat transfer of supercritical fluids in circular tubes using different turbulence models', Journal of Nuclear Science and Technology, 48(3), pp.366-373. 
Zhou, J., Adrian, R.J., Balachandar, S. \& Kendall, T.M., 1999, 'Mechanisms for generating coherent packets of hairpin vortices in channel flow', Journal of Fluid Mechanics, 387, pp.353-396.

Zonta, F., Marchioli, C., \& Soldati, A., 2012, 'Modulation of turbulence in forced convection by temperature-dependent viscosity', Journal of Fluid Mechanics, 697, pp.150-174.

Zonta, F., Onorato, M., \& Soldati, A., 2012, 'Turbulence and internal waves in stablystratified channel flow with temperature-dependent fluid properties', Journal of Fluid Mechanics, 697, pp.175-203.

Zuber, N., 1966, 'An analysis of thermally induced flow oscillations in the near-critical and super-critical thermodynamic region', Technical Report No. NASACR-80609, Research and Development Center, General Electric Company, Schenectady, New York, USA, May 25 


\section{Appendices}

\section{Appendix A Direct Numerical Simulations of Laminar and Turbulent Forced- Convection Heat Transfer in Channels with Supercritical Water}

In this section, direct numerical simulations are used to investigate the forced-convection heat transfer characteristics in channels without the effects of buoyancy, and with water at supercritical thermodynamic state as the working fluid. The aim of work discussed in this section is to report on the relative contributions of laminar- and turbulent-flow mechanisms on IHT and DHT phenomena observed in heat transfer in supercritical fluids. The relevant background information and literature review is presented in Sections 2.2 and 5.1 .

Citation: Azih, C., \& Yaras, M.I., 2013, 'Direct numerical simulations of laminar and turbulent forced-convection heat transfer in channels with supercritical water', The $6^{\text {th }}$ International Symposium on Supercritical Water-cooled Reactors, ISSCWR6-13033 Shenzhen, Guangdong, China, March 03-05.

\section{A.1 Computational Methods}

\section{A.1.1 Test Cases}

Two studies of heat transfer with supercritical working fluids are discussed. The first study aims to investigate the role of wall-normal property variations in supercritical-fluid forced-flow heat transfer in the absence of turbulent mixing. The test cases in this study are referred to as laminar test cases. The second study consisting of turbulent test cases 
investigates the role of inertial phenomena induced by the temperature dependence of thermophysical properties in the modification of flow turbulence, and hence in deviation from subcritical-fluid-based heat transfer predictions. Table A-1 lists the computations performed for the laminar test cases, which are carried out in a laminar base flow at $R e_{d_{h}, \text { in }}=2,000$ and $R e_{d_{h}, \text { in }}=150,000$. The ${ }^{\wedge}$ accent indicates the maximum value of a

Table A-1: $\quad$ Test cases for laminar channel flow

\begin{tabular}{|c|c|c|c|c|}
\hline Case & $\begin{array}{l}\text { Varied fluid } \\
\text { Properties }\end{array}$ & $\begin{array}{c}\dot{Q}_{w}^{\prime \prime} / G \\
(\mathrm{~kJ} / \mathrm{kg})\end{array}$ & $\begin{aligned} & R e_{d_{h}, \text { in }} \\
= & G d_{h} / \mu_{i n}\end{aligned}$ & $\begin{array}{l}\widehat{A C} \\
\times 10^{6}\end{array}$ \\
\hline$L \_Q 0.5 \_\phi f x d$ & None & 0.5 & 2000 & 0 \\
\hline$L \_Q 0.1 \_\phi v a r$ & All & 0.1 & 2000 & 2.400 \\
\hline$L \_Q 0.3 \_\phi v a r$ & All & 0.3 & 2000 & 7.100 \\
\hline$L \_Q 0.5 \_\phi v a r$ & All & 0.5 & 2000 & 12.000 \\
\hline$L \_Q 0.7 \_\phi v a r$ & All & 0.70 & 2000 & 16.800 \\
\hline L_Q0.03_pvar_HighRe & All & 0.03 & 150,000 & 0.009 \\
\hline$L \_Q 0.3 \_\phi \rho$ & $\rho$ & 0.30 & 2000 & 7.100 \\
\hline$L \_Q 0.3 \_\phi \mu$ & $\mu$ & 0.30 & 2000 & 0 \\
\hline$L \_Q 0.3 \_\phi C p$ & $C_{p}$ & 0.30 & 2000 & 0 \\
\hline$L \_Q 0.3 \_\phi k$ & $k$ & 0.30 & 2000 & 0 \\
\hline$L \_Q 0.5 \_\phi \rho$ & $\rho$ & 0.50 & 2000 & 12.000 \\
\hline$L \_Q 0.5 \_\phi \mu$ & $\mu$ & 0.50 & 2000 & 0 \\
\hline$L \_Q 0.5 \_\phi C p$ & $C_{p}$ & 0.50 & 2000 & 0 \\
\hline$L \_Q 0.5 \_\phi k$ & $k$ & 0.50 & 2000 & 0 \\
\hline
\end{tabular}

Constant fluid properties are evaluated at $633 \mathrm{~K}$ and $24 \mathrm{MPa}$ 
Table A-2 Cases for turbulent channel flow

\begin{tabular}{|c|c|c|c|c|c|}
\hline Case & $\begin{array}{l}\text { Varied } \\
\text { fluid } \\
\text { properties }\end{array}$ & $\begin{array}{l}\text { Direction of } \\
\text { gravitational } \\
\text { acceleration }\end{array}$ & $\begin{array}{c}\dot{Q}_{w}^{\prime \prime} / G \\
(\mathrm{~kJ} / \mathrm{kg})\end{array}$ & $\begin{aligned} & R e_{\tau} \\
&= u_{\tau} \delta_{v} \\
& / v\end{aligned}$ & $\begin{array}{c}\widehat{A C} \\
\times 10^{6}\end{array}$ \\
\hline Q0_g0_dfxd & None & - & 0 & 225 & 0 \\
\hline$Q 3 \_g 0 \_\phi f x d$ & None & - & 3.0 & 225 & 0 \\
\hline Q3_g0_фvar & All & - & 3.0 & 225 & 9.8 \\
\hline
\end{tabular}

parameter, and $A c$ is calculated through Equation 5-1. The direct numerical simulations of turbulent flow within a channel are performed at $R e_{d_{h}, i n}=12,000$ and are listed in Table A-2. In the first of these computations named $Q 0 \_g 0 \_\phi f x d$, the flow is developed without heated walls to a statistically-steady turbulent state at a thermodynamic state defined by the temperature and pressure values of $T=643 \mathrm{~K}$ and $p=24 \mathrm{MPa}$, respectively. The results of this simulation serve as an initial condition for subsequent simulations in which the channel walls are heated uniformly such that $\dot{Q}_{w}^{\prime \prime} / G=3 \mathrm{~kJ} / \mathrm{kg}$, yielding a wall temperature variation from $T_{w}<T_{\mathrm{pc}}$ to $T_{w}>T_{p c}$ along the length of the channel surface, where $T_{p c}$ is the temperature at the pseudo-critical point. In the second computation, namely $Q 3 \_g 0 \_\phi f x d$, the fluid properties are fixed at values corresponding to the thermodynamic state in the $Q 0 \_g 0 \_\phi f x d$ test case. Conversely, in the third simulation, namely $Q 3 \_g 0 \_\phi v a r$, the fluid property values are evaluated in accordance with the International Association for the Properties of Water and Steam (IAPWS) equations of state (IAPWS, 2009), and hence accurately represent the supercritical thermodynamic state of water. 


\section{A.1.2 Computational Domains, Boundary Conditions and Initial Conditions}

A schematic of the two-dimensional computational domain used for the laminar test cases is presented in Figure A-1. The length of the computational domain is $3700 \delta$, where $\delta=22.7 \mathrm{~mm}$ is the half-height of the channel, and is chosen to be sufficiently long such that at the outflow boundary the bulk-fluid temperature exceeds the pseudo-critical temperature. In the absence of buoyancy forces, the laminar channel flow is symmetric about the centreline, therefore the wall-normal computational domain dimension is sized to $\delta$, and a symmetry boundary is placed on the upper surface. The fully-developed velocity profile for an isothermal flow in an infinitely wide channel is given by:

$$
u=7.5 \times 10^{-6} \operatorname{Re}_{d_{h}}\left(\frac{y}{2 \delta}-\left(\frac{y}{2 \delta}\right)^{2}\right)(\mathrm{m} / \mathrm{s})
$$

This velocity profile is specified at the inflow boundary and is used to initialize the computational domain. In Equation A-1, the origin is located at the bottom surface as illustrated in Figure A-1, and the multiplying constant, $7.5 \times 10^{-6} \mathrm{~m} / \mathrm{s}$, is obtained from the inflow state of the working fluid. The inlet temperature and pressure are $633 \mathrm{~K}$ and

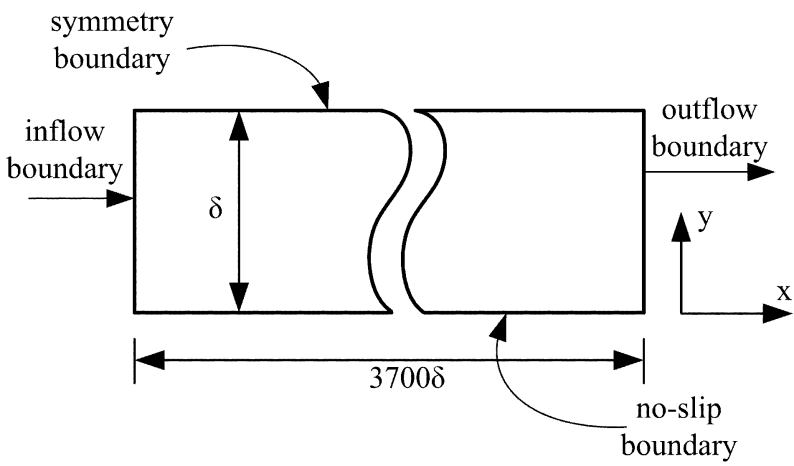

Figure A-1: Schematic of the computational domain for the laminar cases. 
$24 \mathrm{MPa}$, respectively. The flow is heated through a spatially-uniform heat flux applied at the no-slip boundary according to the values specified in Table A-1, and heats the fluid from an inlet bulk-fluid temperature value of $T / T_{p c}=0.97$ to a maximum value of $T / T_{p c}=1.14$ for the highest heat-flux case.

The computational domains for the turbulent test cases are shown in Figure A-2; Figure A-2(a) shows the computational domain for the $Q 0 \_g 0 \_\phi f x d$ test case, and Figure A-2(b) shows the computational domain for the $Q 3 \_g 0 \_\phi f x d$, and $Q 3 \_g 0 \_\phi v a r$ test cases. The channel half-height is set to a value of $\delta=22.7 \mathrm{~mm}$. Heated turbulent flows with supercritical working fluids are strongly affected by near-wall flow phenomena (Azih et al., 2012; Bae et al., 2008; Li et al., 2007). Therefore, the computational domain must be sufficiently long to accurately capture the transient flow

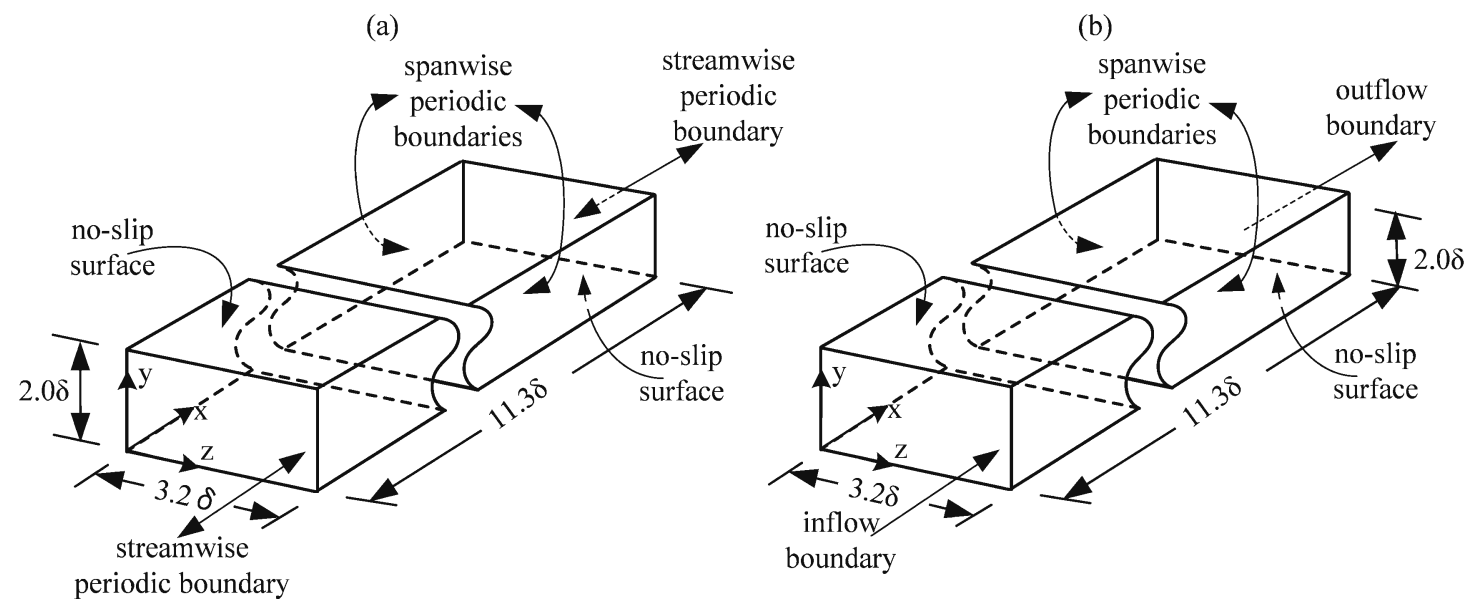

Figure A-2: Computational domains for the turbulent channel cases: (a) computational domain for generating initial conditions for heated-wall channel flow simulations; (b) computational domain for channel flow with heated walls. 
structures developing in the near-wall region. The two computational domains for the turbulent-flow simulations have a streamwise length of $11.3 \delta$, which is sufficient for detailed study of the inner region of the boundary layer defined by $y^{+}<60$ (Jimenez \& Moin, 1991), where the "+" superscript denotes normalization by variables $v$ and $u_{\tau}$, with the thermophysical properties evaluated at the streamwise-local time-averaged wall temperature. Here, $v$ is the kinematic viscosity, and $u_{\tau}$ is the friction velocity defined as $u_{\tau}=\left(\tau_{w} / \rho\right)^{0.5}$, with $\tau_{w}$ being the wall shear stress, and $\rho$ being the density. To ensure selfsustaining turbulence in the channel, the span of the computational domain must adhere to $\Delta z^{+}>220$ (Jimenez \& Moin, 1991). The domain span of $\Delta z=3.2 \delta\left(\Delta z^{+}>740\right)$ is therefore more than sufficient for this study, as it captures multiple instances of the largest near-wall coherent flow structures which have spanwise wavelengths of $\Delta z^{+} \approx 100$ (Panton, 2001). For all of the turbulent test cases, a no-slip boundary condition is applied at the upper and lower surfaces of the channel. At the outflow boundary of the computational domain for the $Q 3 \_g 0 \_\phi f x d$ and $Q 3 \_g 0 \_\phi v a r$ test cases, the static pressure is kept constant at a value of $24 \mathrm{MPa}$ in an area-averaged sense. To eliminate artificial effects of this outflow boundary condition on the flow turbulence, data within $2 \delta$ distance from the outflow boundary is not processed for statistical analysis. To generate inflow data and initial conditions for the turbulent test cases with heated walls, the flow is developed in a streamwise-periodic computational domain at conditions corresponding to the $Q 0 \_g 0 \_\phi f x d$ test case, and from a turbulent initial condition obtained from a recent direct-numerical-simulation study of the author's research group (Azih et al., 2012), until it reaches a statistically-steady turbulent state. At the streamwise-periodic boundaries of 
the $Q 0 \_g 0 \_\phi f x d$ test case, the velocities are mapped between corresponding nodes of the periodic interface, and an area-averaged mass flux value of $7.94 \mathrm{~kg} / \mathrm{s} \mathrm{m}^{2}$ is maintained at the interface, with a sense corresponding to the main flow direction indicated in Fig. 2(a). Once the flow reaches a statistically-steady state, the $Q 0 \_g 0 \_\phi f x d$ simulation is continued for a duration of one through-flow time, and inflow data for the simulations with heated walls is extracted at the plane defined by $x / \delta=9$. The simulations corresponding to the turbulent test cases with heated walls are initialized with velocities corresponding to the final timestep of the $Q 0 \_g 0 \_\phi f x d$ simulation, and are run for several through-flow times; therefore, additional inflow data is required for these test cases. To ensure temporal continuity of the inflow velocity field, the additional inflow velocity data for the turbulent test cases with heated walls is extracted from the $Q 3 \_g 0 \_\phi f x d$ test case at the plane defined by $x / \delta=9$, and a uniform temperature value of $643 \mathrm{~K}$ is specified at the inflow boundary. For the turbulent test cases with heated walls, the area-averaged streamwise velocity at the inlet plane has a value of $0.015 \mathrm{~m} / \mathrm{s}$, which is chosen to meet the target friction Reynolds number of $R e_{\tau}=u_{\tau} \delta / v=225$ at the inflow boundary. A spatially-uniform wall heat flux value is imposed at the no-slip surfaces of the $Q 3 \_g 0 \_\phi f x d$ and Q3_g0_pvar test cases according to the values specified in Table A-2, which heats the flow from a spatially-uniform inlet temperature of $643 \mathrm{~K}$ to a wall temperature value above the pseudo-critical temperature. Spanwise periodic boundaries are applied in all the turbulent test cases, such that all of the primitive variables a mapped between corresponding nodes of this periodic interface 


\section{A.1.3 Spatial and Temporal Grids}

The structured-grid mapped onto the computational domain for the laminar test cases consists of uniformly-spaced nodes in the streamwise direction with a spacing of $\Delta x^{+}=28$, where $v$ and $u_{\tau}$ are evaluated at the inflow boundary. Although the hydrodynamic characteristics of the laminar boundary layer do not scale on the wall variables $\left(v\right.$ and $\left.u_{\tau}\right)$ in the manner as in the inner region of turbulent boundary layers, this normalization is used to facilitate comparison of the computational grid with that of the turbulent test cases of the present study. The nodes are spaced in the wall-normal direction such that the first node off the wall is located at $y^{+}=0.02$, and the node spacing is increased by $8 \%$ up to the channel centreline, located at the top of the computational domain. A simulation was run corresponding to the conditions of $L_{-} 0.7$ all in Table 1, for which the node spacing was halved in the streamwise, spanwise and wall-normal directions, to confirm that the computational results are not sensitive to the grid.

In turbulent channel flow, the smallest eddies are expected to be in the order of the Kolmogorov length scale, $\eta$, which is estimated by $\eta^{+}=\left(0.41 y^{+}\right)^{1 / 4}$ in the log-law region of a constant-property flow, and has a minimum value of $\eta^{+} \approx 2$ at the wall (Stanislas et al., 2008). The grid for the turbulent test cases is sized to be in the order of the Kolmogorov length scale in the wall-normal direction, and has uniform streamwise and spanwise spacings of $\Delta x^{+}=5$ and $\Delta z^{+}=4$, respectively; where $v$ and $u_{\tau}$ are evaluated at the inflow boundary. For the $Q 0 \_g 0 \_\phi f x d$ and $Q 3 \_g 0 \_\phi f x d$ test cases, the first node off the wall is located at $y^{+}=0.25$, and within the $y^{+}$range of $0<y^{+}<20$, the wall-normal node spacing is increased at a constant rate such that there are 17 nodes below the $y^{+}$value of 
10. For $20<y^{+}<150$, the node spacing is established as per the expression for the Kolmogorov length scale for the log-law region, and between $y^{+}=150$ and $y=\delta$ the node spacing is increased at a rate of $10 \%$. In order to accurately capture the temperature field in the Q3 g0_ $\phi v a r$ test case, in the wall-normal direction the nodes are spaced closer together by a factor of at least $\widehat{\operatorname{Pr}}^{1.3}$ (Radjem et al., 2007). With normalization based on the wall variables at the inlet plane, the wall-normal node distribution for the Q3 $\_0 \_\phi v a r$ test case is such that the first node off the wall is located at $y^{+}=0.12$, and within the $y^{+}$range of $0<y^{+}<20$ the wall-normal node spacing is increased at a constant rate such that there are 23 nodes below the $y^{+}$value of 10 . Above $y^{+}=20$, the node spacing for the $Q 3 \_g 0 \_\phi v a r$ test case is equivalent to that of the $Q 3 \_g 0 \_\phi f x d$ test case since, in this region, the node spacing criteria used for the $Q 3 \_g 0 \_\phi f x d$ test case is more than sufficient to resolve both the velocity and temperature fields in the $Q 3 \_g 0 \_\phi v a r$ test case (Friedrich et al., 2001; Radjem et al., 2007; Stanislas et al., 2008).

To accurately capture the temporal development of small-scale turbulence structures in boundary layers, a timestep size of $\Delta t^{+}<0.2$ is required (Friedrich et al., 2001, Kong et al., 2000). For the turbulent test cases, the temporal grid is specified in the form of a constant timestep value of $9 \times 10^{-4} \mathrm{~s}$, which corresponds to $\Delta t^{+}<0.03$, and is dictated by numerical stability as affected by substantial spatial gradients in the thermophysical properties of water near the pseudo-critical point. 


\section{A.1.4 Solution Approach}

For the laminar channel cases, the steady-state solution of the discretized governing equations is obtained by marching the computation in physical time, with one inner-loop iteration per outer-loop iteration, and one outer-loop iteration per timestep. Since the coefficients of the linearized governing equations are not converged within each timestep, the temporal development of the flow is not physically realistic, and the timestep merely serves as a means of under-relaxation as the steady-state solution is computed. Accordingly, larger timesteps may be selected to accelerate convergence to steady state. In addition, the timestep for the laminar cases, which has a value of $\Delta t^{+}=500$ based on the wall variables at the inflow plane, is selected to be large enough to prevent the temporal development of any instabilities in the flow; thus disturbances that would otherwise lead to laminar-to-turbulent transition are damped (Friedrich et al. 2001; Kong et al., 2000). The steady-state computations were deemed to have converged when the temporal gradients vanish, with the root-mean-square of the normalized residuals (ANSYS, 2009) of the computational solution to the governing equations reducing to less than $10^{-7}$.

For the turbulent test cases, the transient solution is obtained with one inner-loop iteration per outer-loop iteration, and multiple outer-loop iterations per timestep. For each timestep, the solution is considered satisfactorily converged when the root-mean-square of the normalized residuals of the computational solution to the governing equations are reduced to less than $10^{-5}$. This is realized with up to five outer-loop iterations per timestep. Approximately 100,000 time steps (1200 viscous time scales) are required to 
reach a statistically-steady state for the $Q 0 \_g 0 \_\phi f x d$ simulation. Using statistically steady results of the $Q 0 \_g 0 \_\phi f x d$ test case as an initial condition, approximately 15,000 and 25,000 timesteps (200, and 300 viscous time scales based on the inflow wall variables) are required to reach a statistically-steady state for the $Q 3 \_g 0 \_\phi f x d$, and $Q 3 \_g 0 \_\phi v a r$ test cases, respectively. After statistically-steady state is achieved, 40,000 and 30,000 timesteps (500 and 400 viscous time scales based on the inflow wall variables) are computed for the $Q 3 \_g 0 \_\phi f x d$, and $Q 3 \_g 0 \_\phi v a r$ test cases, respectively, and collected for analysis.

\section{A.2 Results and Discussion}

\section{A.2.1 Validation of Computational Method}

To validate the numerical approach, results of the $L \_$validation and $Q 3 \_g 0 \_\phi f x d$ test cases are compared to experimental, analytical and computational data available in the published literature.

In the $L$ _validation test case, the flow is heated from a hydrodynamic-fully-developed isothermal inlet profile to a thermally-fully-developed profile. The streamwise-local convection heat transfer coefficient is represented by the Nusselt number, expressed as:

$$
N u_{b}=\frac{4 \delta \dot{Q}_{w}^{\prime \prime}}{k_{b}\left(T_{w}-T_{b}\right)}
$$

Here, $4 \delta$ represents the hydraulic diameter, $k$ is the thermal conductivity, and the subscripts $w$ and $b$ denote properties evaluated at the wall temperature and bulk 
temperature, respectively. The Nusselt number distribution of constant-property laminar flow in an infinitely-wide channel was developed by Cess \& Shaffer (1959), for both thermally-developing and thermally-fully-developed flows. Their results were obtained semi-empirically in the developing region, and a constant value of $N u=8.235$ was obtained analytically for the fully-developed region. These published results are plotted in Figure A-3 along with the results of the L_validation test case. The favourable agreement confirms the accuracy of the computational method used for the laminar test cases. The small discontinuity in the Nusselt number distribution of Cess \& Shaffer (1959), occurring at a bulk fluid enthalpy value of $h_{b} \approx 1770 \mathrm{~kJ} / \mathrm{kg}$, is due to slight underestimation of the thermal entrance length by their semi-empirical correlation.

Validation of the computational method used for the turbulent test cases is described in Section 5.3

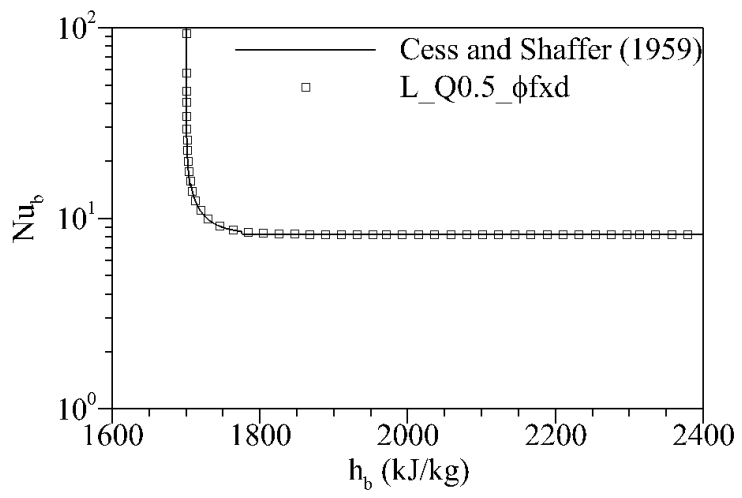

Figure A-3: Streamwise distribution on Nusselt number for fixed-fluid-property channel flows. 


\section{A.2.2 Effect of Wall-Normal Property Variation on Heat Transfer in Laminar}

\section{Flows}

Figure A-4 shows the variation of Nusselt number with the bulk-fluid static enthalpy for the laminar channel cases, in which the heat-flux to mass-flux ratio is varied from a value of $0.1 \mathrm{~kJ} / \mathrm{kg}$ to $0.7 \mathrm{~kJ} / \mathrm{kg}$, and all the fluid properties are varied according to the IAPWS equation of state. In these results, regions are evident in which the Nusselt number either exceeds or falls short of the streamwise distribution of Nusselt number based on a fully developed flow with constant thermophysical fluid properties $(N u=8.235)$. The flow fields that have lower values of the heat- to mass-flux ratio $\left(\dot{Q}_{w}^{\prime \prime} / G\right)$ contain regions of improved heat transfer as indicated by higher $N u$ values, whereas higher values of $\dot{Q}_{w}^{\prime \prime} / G$ result in deteriorated heat transfer. This is similar to the trends seen in turbulent forcedconvection heat transfer involving supercritical fluids (Pioro \& Duffey, 2005). The reasons for these deviations are better understood when the thermophysical properties are

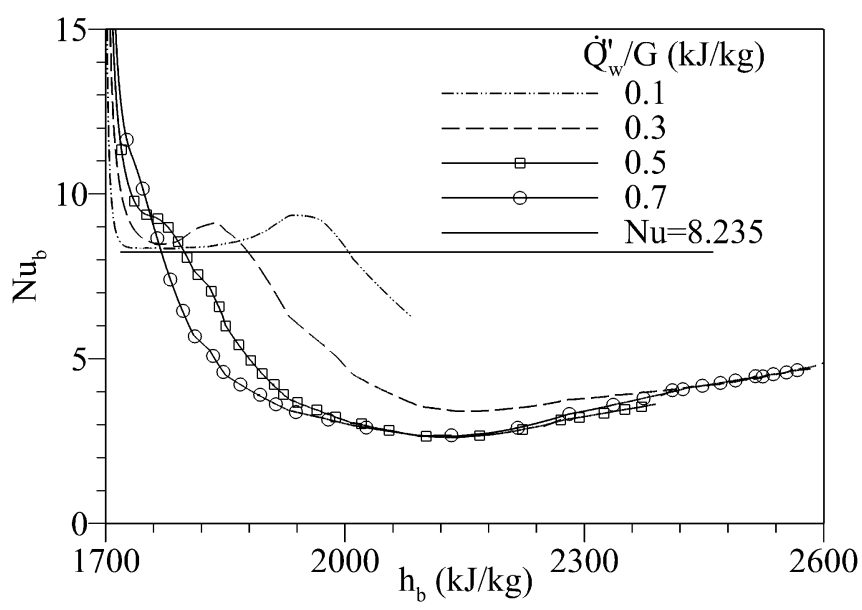

Figure A-4: Nusselt number distributions for a range of various heat-flux to mass-flux ratios in the laminar channel cases; $R e_{d_{h}}=2000$. 
varied systematically, and the results of such an analysis is shown in Figure A-5. The legend in Figure A-5 identifies the sole thermophysical property that is varied for the corresponding case. To establish the physical mechanisms responsible for the observed trends, the streamwise thermal resistance, $R_{x}$, is considered:

$$
R_{x}=\frac{\Delta T_{x}}{\dot{Q}_{w}^{\prime \prime}}=\frac{1}{G A C_{p}}
$$

In this equation, $\Delta T_{x}$ is the streamwise temperature difference, and $A$ is the flow area. Equation A-3 shows that $C_{p}$ directly affects $R_{x}$, whereas the $k$ has no effect on $R_{x}$. Wallnormal gradients in density $(\rho)$ and dynamic viscosity $(\mu)$ modify the velocity profile, and hence affect the distribution of mass flux, $G$, thereby affecting the local value of $R_{x}$. If $R_{x}$ decreases as the wall is approached, the streamwise temperature difference at the wall will decrease relative to that of the bulk fluid. For a given streamwise location, this has the effect of reducing the temperature difference between the wall and the bulk fluid, and thus increasing the value of the local Nusselt number as per Equation A-2.

The trends in Figure A-5 can be readily explained by interpreting the effect that the wallnormal gradient in the varied thermophysical property will have on the flow. The viscosity decreases as the wall is approached, indicated through its temperature dependence illustrated in Figure 1-1, and therefore the resistance to the fluid's inertia is lower near the wall. This causes a net streamwise-momentum transport towards the wall, and thus reduces the thermal resistance to streamwise heat flow near the wall. 

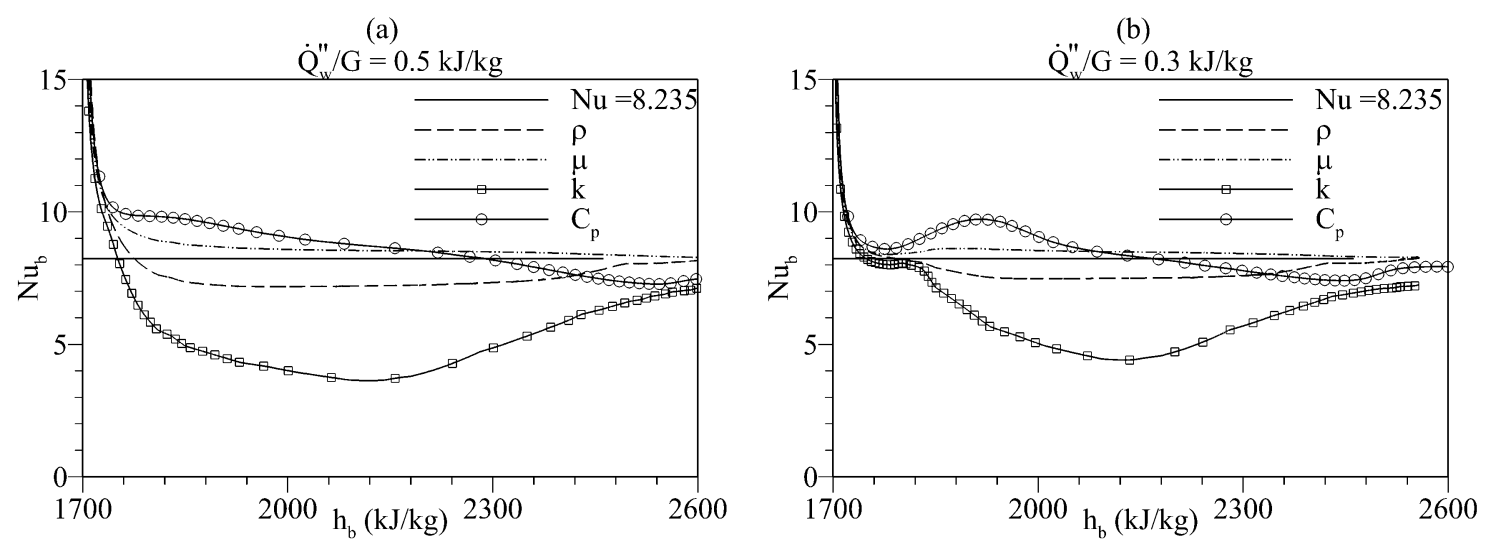

Figure A-5: Effects of thermophysical property variations on the Nusselt number at two heat-flux to mass-flux ratios; $R e_{d_{h}}=2000$.

Conversely, the volumetric expansion, associated with decrease in density as the wall is approached, leads to a net mass transport away from the wall, and hence the near-wall streamwise thermal resistance increases. In the case where only the specific heat is varied, as the flow is heated from a temperature less than $T_{p c}$, the specific heat near the wall increases with streamwise distance causing a reduction in the local thermal resistance to streamwise heat flow, and hence increases the local Nusselt number in comparison to the case of constant-property fluid. However, once the wall temperature exceeds $T_{p c}$, the specific heat of the near-wall fluid starts to decrease with streamwise distance, eventually decreasing below the value of the specific heat of the bulk fluid. This results in a bulk-fluid streamwise thermal resistance that is lower than the thermal resistance of the near-wall fluid, which in turn decreases the value of the local Nusselt number. As the heated wall is approached, the thermal conductivity decreases due to its temperature dependence illustrated in Figure 1-1. Since the transfer of heat through the stationary layer of fluid adjacent to the wall is realized through conduction, for a given wall heat flux, the reduced thermal conductivity of the working fluid at the wall yields an 
increased wall temperature. This results in a decrease in the Nusselt number value as per Equation A-2. As noted in the trends presented in Figure A-5, this effect is significantly larger than the effects of the wall-normal gradients in $\rho, \mu$ and $C_{p}$ on the local Nusselt number.

Van der Kraan et al. (2005) observed heat transfer trends very similar to those of the laminar channel cases of the present study when they investigated a non-buoyant turbulent flow at supercritical thermodynamic state through the solution of the RANS equations with the thermophysical properties of the working fluid varied according to the local thermodynamic state. However, the computational results of van der Kraan et al. did not compare favourably with experimental data. This suggests possible effects of the supercritical thermodynamic state on the turbulent mixing process that are not accounted for by the turbulence closure utilized in the RANS computations of van der Kraan. An analysis of the fixed-property and non-buoyant variable-property turbulent channel cases sheds light on these effects. To enable correlation of any differences in turbulent mixing to the differences in the computed Nusselt number values for the two simulations, the streak spacing values are normalized $\delta$. Table A-3 shows the spanwise streak spacing, Nusselt number, and wall-temperature values evaluated at the plane defined by $x_{\delta}=9$ and at a wall normal location corresponding to the buffer region of the shear layer $\left(y^{+}=30\right)$. The non-buoyant variable-property case has a $35 \%$ reduction in the Nusselt number when compared to the fixed-property case, indicating deteriorated heat transfer (DHT). In 
Table A-3: Nusselt number, streak spacing and wall temperature for turbulent cases $\left(x_{\delta}=9\right)$.

\begin{tabular}{|c|c|c|c|}
\hline Case & Streak spacing, $\Delta \mathrm{z}_{\delta}$ & Wall temperature $(\mathrm{K})$ & Nusselt number \\
\hline$Q^{3} \_g 0 \_\phi f x d$ & 0.44 & 675.7 & 169 \\
\hline Q3_g0_pvar & 1.20 & 685.7 & 125 \\
\hline
\end{tabular}

forced convection turbulent flows, heat transfer is realized through a combination of molecular heat diffusion, and heat advection due to the turbulent motions.

As per the simulation results presented in Figure A-6, a laminar flow environment with wall-normal thermophysical property gradients comparable to that of the $Q 3$ _ $g 0 \_\phi v a r$ turbulent case is obtained. This is realized by running a simulation at a $R e_{d_{h}}=150,000$ and $\dot{Q}_{w}^{\prime \prime} / G=0.03$, with a computational method which ensures that flow instabilities that would otherwise lead to transition are suppressed. This simulation facilitates an analysis of the relative contribution of molecular (laminar), and turbulent advection heat transfer mechanisms, to the overall variation in heat transfer when the flow is in the supercritical
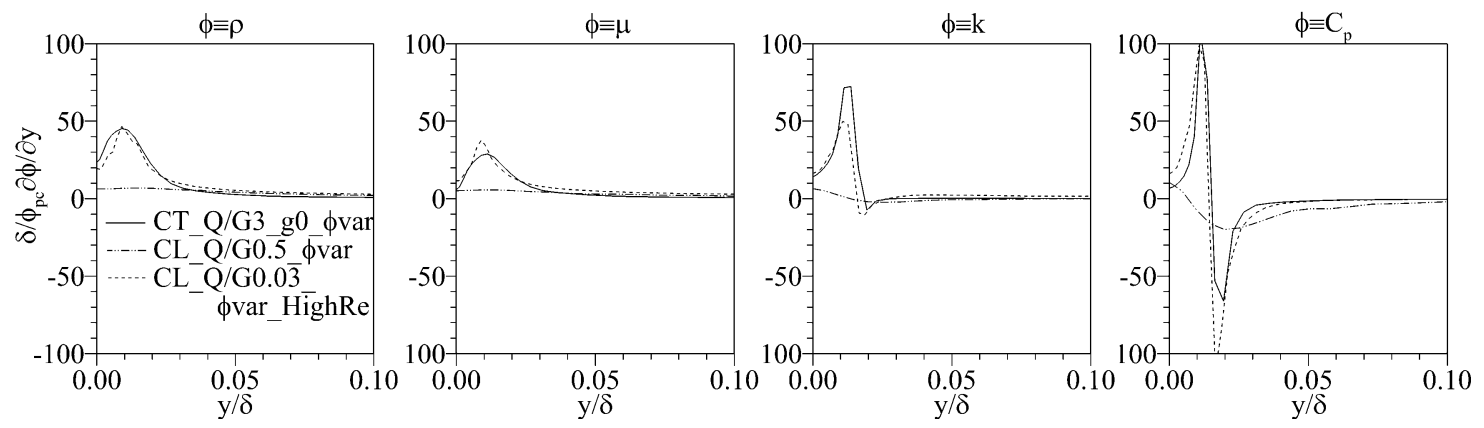

Figure A-6: Wall-normal gradients of thermophysical properties for various cases at a wall temperature value of $685.7 \mathrm{~K}$. 
state. For the High-Re laminar case, the Nusselt number of the laminar-flow is reduced by $25 \%$ compared to the value of a corresponding constant-property laminar flow case. Accordingly, the molecular (laminar) thermal diffusivity in the non-buoyant variableproperty case, which accounts for $15 \%$ of the total thermal diffusivity, as calculated through spatial averaging of thermal diffusivities in the $x=9 \delta$ plane, would be reduced by as much as $25 \%$ (a quarter) due to spatial variations in thermophysical fluid properties. This implies that of the observed $35 \%$ reduction in the Nusselt number in the nonbuoyant variable-property case, about a quarter of $15 \%(\approx 4 \%)$ reduction may be expected directly due to fluid-property variations and no changes in turbulence-related mixing. The remaining Nusselt number reduction of approximately $31 \%$ is therefore due to the effects of thermophysical property variations on turbulent mixing. This clearly illustrates the dominant role of turbulence phenomena in the heat transfer variations observed with supercritical fluids. The large change in the spanwise streak spacing between the fixedproperty and non-buoyant variable-property turbulent cases suggest that there is a substantial change in the physical topology of the flow structures of turbulence between the two cases. 


\section{Appendix B Numerical and Computational Method}

In the present study, heated flow of supercritical fluids over flat surfaces and within rectilinear channels are studied by numerically solving the governing equations for fluid flow using the DNS method. DNS requires computational grids that are sufficiently refined to capture all of the spatial and temporal scales of the relevant flow phenomena, such that semi-empirical models are not needed to aid in closure of the governing equations that are filtered in some sense.

This thesis is written in an integrated-thesis format, therefore, the details of the flow conditions for the studied cases and the spatial and temporal grids employed for each case

are discussed in detail in the relevant results chapters. In this chapter, the governing equations are first outlined, and then the discretization scheme for these governing equations is explained. A general overview of boundary conditions and mesh generation are presented in Sections B.3and B.4, respectively. Finally, the data reduction methods are described.

\section{B.1 Governing Equations of Fluid Flow}

The commercial computational fluid dynamics software package ANSYS CFX® (Version 12) is used to perform the simulations of the present study. Using a finite volume technique, the solver discretizes the strong conservation-law form of the governing equations given in tensor notation in Equations B-1 to B-3 (ANSYS, 2009). 


$$
\begin{gathered}
\frac{\partial \rho}{\partial t}+\frac{\partial}{\partial x_{j}}\left(\rho u_{j}\right)=0 \\
\frac{\partial}{\partial t}\left(\rho u_{j}\right)+\frac{\partial}{\partial x_{j}}\left(\rho u_{j} u_{i}\right)=-\frac{\partial p}{\partial x_{i}}+\frac{\partial \tau_{i j}}{\partial x_{j}}+\rho g_{i}+M_{i} \\
\frac{\partial}{\partial t}\left(\rho h_{0}\right)+\frac{\partial}{\partial x_{j}}\left(\rho u_{j} h_{0}\right)=\frac{\partial p}{\partial t}+\frac{\partial}{\partial x_{j}}\left(k \frac{\partial T}{\partial x_{j}}\right)+\frac{\partial}{\partial x_{j}}\left(u_{i} \tau_{i j}\right)+\rho u_{i} g_{i}
\end{gathered}
$$

In these equations, $u_{j}$ is the $j$ component of velocity, $x_{j}$ is the $j$ component of Cartesian spatial coordinate, $\tau_{i j}$ is the $i j$ component of the viscous stress tensor, $h_{0}$ is the total enthalpy, $T$ is the static temperature, and $M$ represents external momentum sources. The fluid is modelled as a Newtonian fluid such that the components of the viscous stress tensor relate to the strain rates through:

$$
\tau_{i j}=\mu\left(\frac{\partial u_{i}}{\partial x_{j}}+\frac{\partial u_{j}}{\partial x_{i}}\right)
$$

Inherent in the formulation of Equations B-2 to B-4 is stokes hypothesis which effectively ignores the contribution of the bulk viscosity on the flow development. For wall-bounded turbulent flows, Cramer \& Bahmani (2014) demonstrate that this hypothesis is only valid if the ratio of the bulk viscosity to dynamic viscosity is less than $\left(R e_{\delta}\right)^{0.5}$. For the $p / p_{c}$ value of 1.085 for the simulations of the present study, the maximum value of the ratio of bulk viscosity to wall viscosity is approximately 50 , and occurs at the pseudo-critical state (Hasan \& Farouk, 2012). This value is about half of $\left(R e_{\delta}\right)^{0.5}$, therefore it is expected that the effects of bulk viscosity are negligible under the conditions of the present study.

The governing equations are coupled with the working fluid's equations of state to form a closed system of equations. When air is used as the working fluid, it is assumed that its 
thermodynamic state can be approximated by the perfect gas model with $C_{p}=1.0044 \mathrm{~kJ} /(\mathrm{kg} \mathrm{K})$. When water is used water as the working fluid, the International Association for the Properties of Water and Steam (IAPWS) equations of state are applied (IAPWS, 2009; IAPWS, 2008). The solver converts the IAPWS equation of state, which expresses the thermodynamic properties as function of temperature and pressure, into tables indexed by pressure and enthalpy. For each simulation, the pressure range selected for generating the fluid-property tables span over ten times the predicted range of pressure variations (White, 1991; Pope, 2000; Kasagi \& Shikazono 1995). Similarly, the temperature range for the tables is chosen to be at least four times the range of temperatures expected in the flow (Pioro ${ }^{b}$ et al., 2004; Kasagi \& Shikazono, 1995). The tables are generated with temperature and pressure increments of $\Delta T \leq 0.2 \mathrm{~K}$ and $\Delta p \leq 0.08 \mathrm{MPa}$, respectively. The solver obtains relevant thermodynamic properties through linear interpolation of the tabularized provides values of all with and accuracy that is comparable to the uncertainty associated with IAPWS equations of state within the supercritical region.

\section{B.2 Discretization Scheme}

The governing equations are discretized using an element-based finite-volume technique with a coupled algebraic multigrid algorithm used to solve the linear system of algebraic equations that result from the discretization scheme (ANSYS 2009). Global property conservation is ensured by enforcing local conservation over control volumes surrounding each node in the computational domain, while fluid-property variations 
across the surfaces of the control volumes are captured using finite-element-based shape functions (ANSYS, 2009).

\section{B.2.1 Finite Volume Discretization of Governing Equations}

The governing and thermodynamic state equations are integrated over the control volumes created using the mesh elements and all flow and fluid variables are stored at the corresponding mesh node. With reference to Figure B-1, the control volumes are defined by the element centers which are determined by the intersection of lines drawn across the midpoints of the edges of the segments within the element. The present study utilizes regular hexahedral elements, however, Figure B-1 illustrates the element in a twodimensional schematic for simplicity.

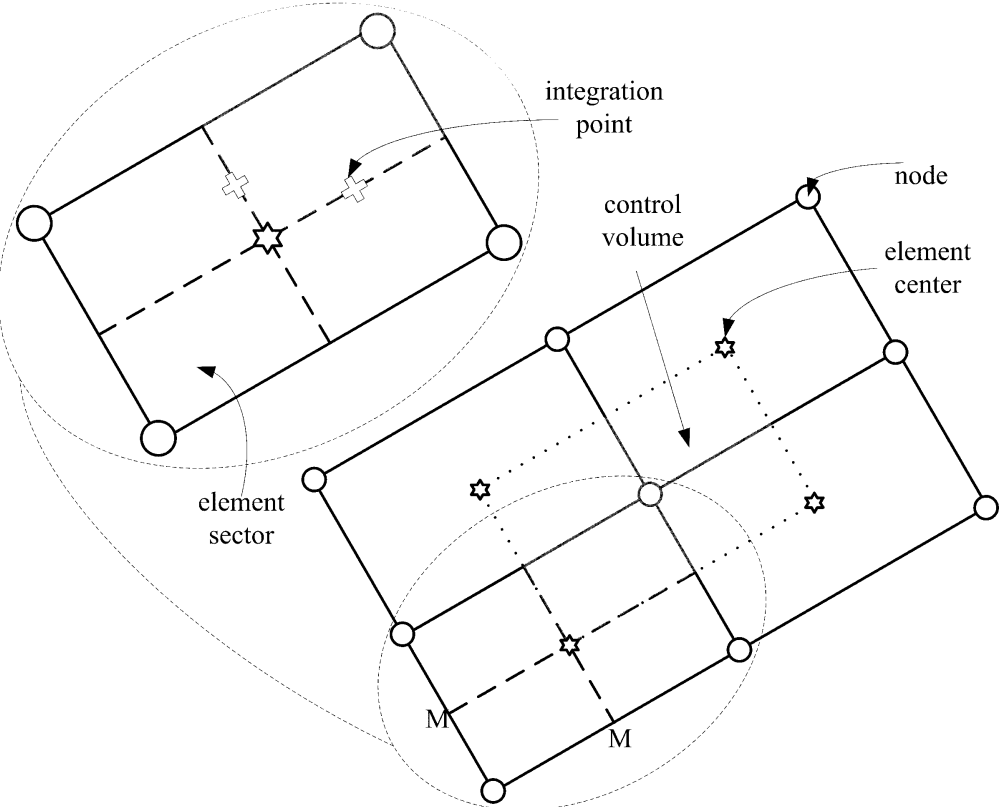

Figure B-1: Schematic of a two-dimensional mesh showing discretization nomenclature (Adapted from ANSYS, 2009). 
When integrating the governing equations over the control volume, the volume integrals involving fluid-property divergence and fluid-property gradients are converted to surface integrals using Gauss' divergence theorem, Thus Equations B-1 to B-3 become:

$$
\begin{gathered}
\frac{\partial}{\partial t} \int_{V} \rho d V+\int_{S} \rho u_{j} d n_{j}=0 \\
\frac{\partial}{\partial t} \int_{V} \rho u_{j} d V+\int_{S} \rho u_{j} u_{i} d n_{j}=-\int_{S} p d n_{j}+\int_{S} \tau_{i j} d n_{j}+\int_{V} \rho g_{i} d V+\int_{V} \boldsymbol{M}_{i} d V \\
\frac{\partial}{\partial t} \int_{V} \rho h_{0} d V+\int_{S} \rho u_{j} h_{0} d n_{j} \\
=\frac{\partial}{\partial t} \int_{V} p d V+\int_{S} k \frac{\partial T}{\partial x_{j}} d n_{j}+\int_{S} u_{i} \tau_{i j} d n_{j}+\int_{V} \rho u_{i} g_{i} d V
\end{gathered}
$$

In these equations $V$ represents the control volume, $s$ represents the surfaces of the control volume and $n$ is the outward-normal surface vector. Volume integrals are discretized within element sectors, and the integral values for each element sector are combined to obtain the value for the control volume. The surface integrals are discretized about the integration points. This yields Equations B-8 to B-10 as the discretized from of Equations B-5 to B-7.

$$
\begin{gathered}
V_{c v} \frac{\Delta \rho}{\Delta t}+\sum_{i p}\left(\rho u_{j} \Delta n_{j}\right)_{i p}=0 \\
V_{c v} \frac{\Delta\left(\rho u_{i}\right)}{\Delta t}+\sum_{i p}\left(\rho u_{j} \Delta n_{j}\right)_{i p}\left(u_{i}\right)_{i p} \\
=-\sum_{i p}\left(p \Delta n_{i}\right)_{i p}+\sum_{i p}\left(\tau_{i j} d n_{j}\right)_{i p}+V_{c v} \rho g_{i}+V_{c v} M_{i}
\end{gathered}
$$




$$
\begin{aligned}
V_{c v} \frac{\Delta\left(\rho h_{0}\right)}{\Delta t}+\sum_{i p}\left(\rho u_{j} h_{0} \Delta n_{j}\right)_{i p} & \\
& =V_{c v} \frac{\Delta p}{\Delta t}+\sum_{i p}\left(k \frac{\partial T}{\partial x_{j}} \Delta n_{j}\right)_{i p}+\sum_{i p}\left(u_{i} \tau_{i j} \Delta n_{j}\right)_{i p} \quad \text { B-10 } \\
& +V_{c v} \rho u_{i} g_{i}
\end{aligned}
$$

here $\Sigma_{i p}$ indicates summation over all the integration points within the control volume.

\section{B.2.2 Order of Accuracy of Discretization Schemes}

To avoid clutter, the terms in Equations B-8 to B-10 that still require further specification, are treated though a representative variable, $\phi$.

The discretization of the advection terms (e.g. second term in Equation B-9) is such that the value of $\phi$ at the integration point must be approximated. This approximation is implemented using nodal values from an upstream integration point, up, as follows:

$$
\phi_{i p}=\phi_{u p}+\frac{\partial \phi}{\partial x_{i}} \cdot \Delta x_{i}
$$

In Equation B-11, $\Delta x_{i}$ represents the position vector from the upstream integration point to the evaluated integration point, the gradient term represents the local element gradient of $\phi$, thus, Equation B-11 represents the second-order accurate centered-difference scheme. Spatial gradients are approximated using tri-linear finite element shape functions. The nature and implementation of the shape functions are described in the ANSYS CFX Solver Theory Guide (ANSYS, 2009). 
The solver handles diffusion terms through the standard finite element approach that utilizes shape functions to evaluate the spatial derivatives for the diffusion terms. For example, a derivative of $\phi$ in the $x$-spatial direction at ip is approximated by:

$$
\left.\left.\frac{\partial \phi}{\partial x}\right]_{i p}=\sum_{n} \frac{\partial N_{n}}{\partial x}\right]_{i p} \phi_{n}
$$

Where $N$ is the shape function.

Transient terms are discretized using the second-order accurate backward Euler method, yielding:

$$
\frac{\Delta \phi}{\partial t}=\frac{1}{2 \Delta t}\left(3 \phi^{t}-4 \phi^{t-1}+\phi^{t-2}\right)
$$

Where the superscripts $t, t-1$, and $t-2$ represent the current, and previous two timesteps respectively.

\section{B.2.3 Solution Method for the Linearized System of Equations}

Application of the discretization scheme to all elements in the domain results in a linearized system of equations that is solved using a multigrid accelerated Incomplete Lower Upper (ILU) factorization technique. In matrix form, the linearized system of equations can be written as:

$$
[C][\phi]=[b]
$$

Where $[C]$ is the coefficient matrix, $[\phi]$ is the solution vector and $[b]$ represents coefficients of the right-hand-side vector. To reduce the amount of arithmetic operations and hence physical memory required to solve the above equation, the ILU factorization technique is favoured over direct techniques, however, this comes at a price of requiring 
several iterations to approach the exact solution to Equation B-14. The iterative method is implemented by starting with an approximate solution, $\left[\phi^{n}\right]$, which is corrected by $\left[\phi^{c}\right]$, to yield a new solution, $\left[\phi^{n+1}\right]$.

$$
[\phi]^{n}+[\phi]^{c}=[\phi]^{n+1}
$$

Substitution of Equation B-15 into B-14 yields and equation for the residual, re, to the current solution iteration.

$$
[C][\phi]^{c}=[b]-[C][\phi]^{n} \equiv r e^{n}
$$

Iterations on Equation B-16, referred to as inner-loop iterations, cause the correction term to tend towards zero; thus, approaching the actual solution to the discretized set of equations. For transient problems, a solution at a given timestep is deemed as converged when the normalized residual $r e^{*, n}$, defined for the $m^{\text {th }}$ node, attains a sufficiently low value as:

$$
r e_{m}^{*, n}=r e_{m}^{n} /\left(c_{m} \Delta \phi\right)
$$

here, $c_{m}$ is central coefficient of the discretized control volume equation, and $\Delta \phi$ represents the degree of variation of $\phi$.

The ILU factorization solution technique is only efficient at reducing errors with wavelengths on the order of the mesh spacing. Thus, a multigrid technique for which a solution is also computed on progressively coarser meshes can significantly improve the efficiency of the solver. The solver uses an Algebraic Multigrid technique in which iterations are cycled between finer and coarser grids indirectly constructed by algebraic manipulation of the system of linearized equations (Raw, 1996). In particular, the Additive Correction Multigrid method of Hutchinson \& Raithby (1986) is used to 
generate a discrete system of equations for a coarser meshes by summing the fine mesh equations, and thus, merging multiple neighbouring control volumes to create larger volumes. Calculations corresponding to the finer meshes more effectively reduce the shorter wavelength errors while the longer wavelength errors are efficiently dealt with on coarser grids. For each solution iteration, several passes between each grid level may be used, and the number of passes is defined by the multigrid cycle. ANSYS CFX® uses fixed W-type algebraic multigrid cycles. Six grid levels are used in the multigrid cycle. A single solution sweep is performed for each grid level during the restriction legs of the multigrid cycle, and three solution sweeps are performed for each grid level during the prolongation legs. Further details on this technique can be obtained from the ANSYS CFX® ${ }^{\circledR}$ solver theory guide (ANSYS, 2009).

The solution convergence of the discretized equations at each timestep is obtained with outer iteration loops serving to converge the coefficients of the linearized governing equations, and inner iteration loops facilitating the solution of these equations through $\mathrm{W}$ type algebraic multigrid cycles. For steady-state problems, the solution is marched in physical time until the transient terms approach zero. Here, one inner-loop iteration is performed per outer-loop iteration, and one outer-loop iteration per timestep. Since the coefficients of the linearized governing equations are not converged within each timestep, the temporal development of the flow is not physically realistic, and the timestep merely serves as a means of under-relaxation as the steady-state solution is computed. Therefore, when a steady-state solution is desired, the transient terms are not included in the normalized residual calculation described by Equation B-17. The transient solution is 
obtained with one inner-loop iteration per outer-loop iteration, and multiple outer-loop iterations per timestep. For each timestep, the solution is considered satisfactorily converged when the root-mean-square of the residuals of the computational solution to the governing equations are reduced to less than a specified value.

\section{B.3 Boundary and Initial Conditions}

For the given governing equations and computational domains, the development of the flow in space and time is determined by the applied boundary and initial conditions. In the present study, no-slip surfaces, free-slip surfaces, surfaces with spatially-uniform heat fluxes, adiabatic surfaces, and periodic boundaries are specified as boundary condition on the computational domain. The solver imposes the no-slip condition by enforcing the velocity at the nodes on the surface to be zero. At a free-slip surface, a zero value for the wall-normal velocity and shear stress is enforced. A numerical value for the heat flux at a given surface may be specified, with a positive value indicating thermal energy is being transferred into the computational domain. For adiabatic surface the heat flux value is set to zero. Periodic boundaries are used extensively in this study to reduce the computational resources required to simulate the test cases of the present study at their respective target Reynolds numbers. A translational periodic boundary condition may be applied to parallel surfaces such that the primitive solution variables (pressures, velocities, and temperatures) are mapped between corresponding nodes on the periodic surfaces. For streamwise-homogeneous flows, a mass flow rate can be specified at the 
periodic boundary allowing the solver to eliminate the pressure from the mapped variables.

Some caution must be applied when mapping an initial flow field to computational domains that have streamwise-periodic boundaries. Under such conditions, the initial conditions also serve as the effective inflow boundary condition. For flows without streamwise-periodic boundary conditions, the initial conditions would ideally be specified using the best approximation of the expected solution field to decreases the likelihood of divergence of the numerical algorithm and reduce the simulation time required to obtain an adequate solution field.

\section{B.4 Mesh Generation}

An in-house software developed by Brinkerhoff (2014) is used to generate the computational domains and spatial grids in the present study. The software, called PatranMeshGenerator, is written in ANSI C. It creates a structured, Cartesian grid formatted as a Patran Neutral file, which can be imported into ANSYS CFX and other commercial Computational Fluid Dynamics (CFD) solver. Details on the coding and implementation of the software are recorded by Brinkerhoff (2014).

\section{B.5 Data Reduction}

This section describes the data reduction processes that were used in the present study. The data extracted from the solution of each case constitute the components of the 
velocity field $(u, v, w)$, the static pressure $(p)$, and the static temperature $(T)$. Manipulation of this data included differentiation and integration of flow variables, averaging along temporal and spatial coordinates, and conducting wave-number analyses.

\section{B.5.1 Differentiation Methodology}

Differentiation operations were computed using a second-order accurate scheme. The partial derivative of a given parameter, $\phi$, along a coordinate, $x$, at a given node defined by the index $i$, is obtained by taking the derivative of a quadratic curve-fit defined using $\left(\phi_{i}, x_{i}\right)$ and two neighbouring points. When node $i$ is not at a boundary along the given coordinate, a centred-differencing scheme is employed:

$$
\begin{gathered}
\left.\frac{\partial \phi}{\partial x}\right|_{i}=\phi_{i-1} \frac{x_{i}-x_{i+1}}{\left(x_{i-1}-x_{i}\right)\left(x_{i-1}-x_{i+1}\right)}+\phi_{i} \frac{-x_{i-1}+2 x_{i}-x_{i+1}}{\left(x_{i}-x_{i-1}\right)\left(x_{i}-x_{i+1}\right)} \\
+\phi_{i+1} \frac{x_{i}-x_{i-1}}{\left(x_{i-1}-x_{i+1}\right)\left(x_{i}-x_{i+1}\right)}
\end{gathered}
$$

at boundary nodes, derivatives are evaluated using the respective forward- and backwarddifferencing schemes as applicable:

$$
\begin{gathered}
\left.\frac{\partial \phi}{\partial x}\right|_{i}=\phi_{i} \frac{2 x_{i}-x_{i+1}-x_{i+2}}{\left(x_{i}-x_{i+1}\right)\left(x_{i}-x_{i+2}\right)}+\phi_{i+1} \frac{x_{i}-x_{i+2}}{\left(x_{i+1}-x_{i}\right)\left(x_{i+1}-x_{i+2}\right)} \\
\quad+\phi_{i+2} \frac{x_{i}-x_{i+1}}{\left(x_{i}-x_{i+2}\right)\left(x_{i+1}-x_{i+2}\right)} \\
\left.\frac{\partial \phi}{\partial x}\right|_{i}=\phi_{i} \frac{2 x_{i}-x_{i-1}-x_{i-2}}{\left(x_{i-2}-x_{i}\right)\left(x_{i-1}-x_{i}\right)}+\phi_{i-1} \frac{x_{i}-x_{i-2}}{\left(x_{i-1}-x_{i}\right)\left(x_{i-1}-x_{i-2}\right)} \\
+\phi_{i-2} \frac{x_{i}-x_{i-1}}{\left(x_{i-2}-x_{i-1}\right)\left(x_{i-2}-x_{i}\right)}
\end{gathered}
$$




\section{B.5.2 Integration Methodology}

The integral of a parameter, $\phi$, over the time coordinate is given in Equation B-21 as an example of the methodology used. At a spatial coordinate defined by $x_{0}, y_{0}, z_{0}$ and for a time duration bounded by $t=t_{0}$ to $t=t_{N}$, the integral of a variable $\phi$ is approximated as:

$$
\int_{t=t_{0}}^{t=t_{N}} \phi\left(x_{0}, y_{0}, z_{0}, t\right) d t \approx \sum_{i=0}^{i=N-1} \phi\left(x_{0}, y_{0}, z_{0}, t_{i}\right)\left(t_{i+1}-t_{i}\right)
$$

where $i=0,1,2, \ldots . . N$.

\section{B.5.3 Averaging Methodology}

The time average of a generic variable, $\phi$, over duration bounded by $t=t_{0}$ to $t=t_{N}$ and at a spatial coordinate defined by $x_{0}, y_{0}, z_{0}$ is given by:

$$
\overline{\phi\left(x_{0}, y_{0}, z_{0}, t\right)}=\frac{\int_{t=t_{0}}^{t=t_{N}} \phi\left(x_{0}, y_{0}, z_{0}, t\right) d t}{\int_{t=t_{0}}^{t=t_{N}} d t}
$$

The integrations are performed using the methodology described in Section B.5.2.

Likewise, at a given instance in time, $t_{0}$, the average taken along a spatial coordinate, e.g. $z$ over a range $z_{0}$ to $z_{N}$, is defined as:

$$
\overline{\phi\left(x_{0}, y_{0}, z, t_{0}\right)}=\frac{\int_{z=z_{0}}^{z=z_{n}} \phi\left(x_{0}, y_{0}, z, t_{0}\right) d z}{\int_{z=z_{0}}^{z=z_{N}} d z}
$$

The Favre average of a variable is the density weighted average of the variable. For example, this average taken over the time coordinate is given by:

$$
\phi\left(x_{0}, \widetilde{y_{0}}, z_{0}, t\right)=\frac{\int_{t=t_{0}}^{t=t_{N}} \rho\left(x_{0}, y_{0}, z_{0}, t\right) \phi\left(x_{0}, y_{0}, z_{0}, t\right) d t}{\int_{t=t_{0}}^{t=t_{N}} \rho\left(x_{0}, y_{0}, z_{0}, t\right) d t}
$$

Likewise, over a spatial coordinate, e.g. the $z$ coordinate, the Favre average is given by: 


$$
\phi\left(x_{0}, \widetilde{y_{0}, z}, t_{0}\right)=\frac{\int_{z=z_{0}}^{z=z_{N}} \rho\left(x_{0}, y_{0}, z, t_{0}\right) \phi\left(x_{0}, y_{0}, z, t_{0}\right) d z}{\int_{z=z_{0}}^{z=z_{N}} \rho\left(x_{0}, y_{0}, z, t_{0}\right) d z}
$$

In the present study, area averages were taken along several planar surfaces. For example, over the $y-z$ plane the area average is defined as:

$$
\overline{\phi\left(x_{0}, y, z, t_{0}\right)}=\frac{\int_{y=y_{0}}^{y=y_{N}} \int_{z=z_{0}}^{z=z_{N}} \phi\left(x_{0}, y, z, t_{0}\right) d z d y}{\int_{y=y_{0}}^{y=y_{N}} \int_{z=z_{0}}^{z=z_{N}} d z d y}
$$

If the averages above were obtained along at least one coordinate where the turbulence field was not statistically homogeneous, then the average is indicated by angled brackets $\langle\phi\rangle$. The subscript, $b$, is reserved for averages computed to obtain bulk-fluid properties. To obtain bulk-fluid thermophysical properties the mass-weighted area-averaged of the enthalpy is obtained at the given streamwise location, then the relevant properties are evaluated at this enthalpy and the area-averaged pressure. For example to obtain bulkfluid properties over a given $y-z$ plane at a given streamwise location, $x_{0}$, and a given instance in time, $t_{0}$ :

$$
\begin{gathered}
h_{b}\left(x_{0}, y, z, t_{0}\right)=\frac{\int_{y=y_{0}}^{y=y_{N}} \int_{z=z_{0}}^{z=z_{N}} \rho\left(x_{0}, y, z, t_{0}\right) h\left(x_{0}, y, z, t_{0}\right) d z d y}{\int_{y=y_{0}}^{y=y_{N}} \int_{z=z_{0}}^{z=z_{N}} \rho\left(x_{0}, y, z, t_{0}\right) d z d y} \\
p_{b}\left(x_{0}, y, z, t_{0}\right)=\frac{\int_{y=y_{0}}^{y=y_{N}} \int_{z=z_{0}}^{z=z_{N}} p\left(x_{0}, y, z, t_{0}\right) d z d y}{\int_{y=y_{0}}^{y=y_{N}} \int_{z=z_{0}}^{z=z_{N}} d z d y}
\end{gathered}
$$

Then,

$$
\phi_{b}=\phi\left(h_{b}, p_{b}\right)
$$

\section{B.5.4 Spectral Analyses Methodology}

Spectral analysis of the simulation results are used to identify the frequency and wave number distributions of the kinetic energy in the turbulent motions. A fast Fourier 
transform (FFT) algorithm is used to transform time or spatial-traces of specified variables to the spectral domain and calculate the power spectral density (PSD) distributions within the simulation results. The FFT algorithm built into the computer coding software Matlab ${ }^{\circledR}$ was used in the present work. This FFT algorithm is based on the work of Cooley-Tukey (1965).

\section{B.5.5 Vortex Identification Methodology}

Lesieur et al. (2005) define coherent vortical structures of turbulence as flow regions having a high concentration of vorticity with a low static pressure core, such that the fluid particles rotating about the vortex are relatively balanced between centrifugal and pressure gradient forces. Dubief \& Delcayre (2000) and Lesieur et al. (2005) mathematically demonstrate that a necessary condition for the presence of a vortex tube with a low-pressure core is a positive value of the second invariant of the velocity gradient tensor, denoted by $q$. Brinkerhoff \& Yaras (2014) determined that the vortical structures of turbulence structures are most clearly visualized when $q$ is calculated using the perturbation velocity flow field. In the present study, $q$ was calculated as:

$$
q=\frac{1}{2}\left(\frac{\partial u_{i}}{\partial x_{i}} \frac{\partial u_{j}}{\partial x_{j}}-\frac{\partial u_{i}}{\partial x_{j}} \frac{\partial u_{j}}{\partial x_{i}}\right)
$$

where Einstein summation is implied over the indexed terms. 


\section{Appendix C Derivation of Non-Dimensional Forms of the Governing Equations and \\ Boundary Conditions}

\section{C.1 Derivation of Non-Dimensional Continuity Equation}

Conservation of mass (conservation form)

$$
\frac{\partial}{\partial t} \rho+\frac{\partial}{\partial x_{i}}\left(\rho u_{i}\right)=0
$$

let

$$
u^{*}=\frac{u}{u_{r e f}}, \quad x^{*}=\frac{x}{x_{\text {ref }}}, \quad t^{*}=\frac{t}{x_{\text {ref }} / u_{\text {ref }}}, \quad \rho^{*}=\frac{\rho}{\rho_{\text {ref }}}
$$

Multiply Equation C-1 by $x_{\text {ref }} f\left(\rho_{r e f} u_{r e f}\right)$

$$
\frac{\partial\left(\frac{\rho}{\rho_{\text {ref }}}\right)}{\partial\left(\frac{t}{x_{r e f} / u_{r e f}}\right)}+\frac{\partial}{\partial\left(\frac{x}{x_{r e f}}\right)}\left(\frac{\rho}{\rho_{\text {ref }}} \frac{u}{u_{r e f}}\right)=0
$$

$$
\frac{\partial}{\partial t^{*}} \rho^{*}+\frac{\partial}{\partial x_{i}^{*}}\left(\rho^{*} u_{i}^{*}\right)=0
$$

\section{C.2 Derivation of Non-Dimensional Momentum Equation}

Conservation of momentum (conservation form)

$$
\frac{\partial}{\partial t}\left(\rho u_{i}\right)+\frac{\partial}{\partial x_{j}}\left(\rho u_{i} u_{j}\right)=-\frac{\partial}{\partial x_{i}} p+\frac{\partial}{\partial x_{j}} \tau_{i j}+\rho g_{i}
$$

multiply Equation C-5 by $1 / \rho_{\text {ref }}$

$$
\frac{\partial}{\partial t}\left(\rho^{*} u_{i}\right)+\frac{\partial}{\partial x_{j}}\left(\rho^{*} u_{i} u_{j}\right)=-\frac{1}{\rho_{r e f}} \frac{\partial}{\partial x_{i}} p+\frac{1}{\rho_{r e f}} \frac{\partial}{\partial x_{j}} \tau_{i j}+\rho^{*} g_{i} \quad \text { C-6 }
$$

multiply Equation C- 6 by $1 / u_{\text {ref }}$ 


$$
\frac{\partial}{\partial t}\left(\rho^{*} u_{i}^{*}\right)+\frac{\partial}{\partial x_{j}}\left(\rho^{*} u_{i}^{*} u_{j}\right)=-\frac{1}{\rho_{\text {ref }} u_{r e f}} \frac{\partial p}{\partial x_{i}}+\frac{1}{\rho_{\text {ref }} u_{r e f}} \frac{\partial}{\partial x_{j}} \tau_{i j}+\frac{\rho^{*} g_{i}}{u_{r e f}}
$$

multiply Equation C-7 by $x_{\text {ref }} / u_{\text {ref }}$ and let:

$$
p^{*}=\frac{p}{\rho_{r e f} u_{r e f}^{2}}, \quad R i=\frac{g_{i} x_{r e f}}{u_{r e f}^{2}}
$$

then

$$
\frac{\partial}{\partial t^{*}}\left(\rho^{*} u_{i}^{*}\right)+\frac{\partial}{\partial x_{j}^{*}}\left(\rho^{*} u_{i}^{*} u_{j}^{*}\right)=-\frac{\partial p^{*}}{\partial x_{i}^{*}}+\frac{\partial}{\partial x_{j}^{*}} \frac{1}{\rho_{r e f} u_{r e f}^{2}} \tau_{i j}+\rho^{*} R i
$$

Examining the shear force term in Equation C-9:

$$
\frac{1}{\rho_{r e f} u_{r e f}^{2}} \tau_{i j}=\frac{\mu}{\rho_{r e f} u_{r e f}^{2}}\left(\frac{\partial u_{i}}{\partial x_{j}}+\frac{\partial u_{j}}{\partial x_{i}}\right) \times \frac{x_{r e f}}{x_{r e f}} \frac{\mu_{r e f}}{\mu_{r e f}}
$$

let

$$
\mu^{*}=\frac{\mu}{\mu_{r e f}}, \quad R e=\frac{\rho_{r e f} u_{r e f} x_{r e f}}{\mu_{r e f}}
$$

then

$$
\frac{1}{\rho_{\text {ref }} u_{r e f}^{2}} \tau_{i j}=\frac{\mu^{*}}{R e}\left(\frac{\partial u_{i}^{*}}{\partial x_{j}^{*}}+\frac{\partial u_{j}^{*}}{\partial x_{i}^{*}}\right)=\tau_{i j}^{*}
$$

When buoyancy forces are significant, Equation C-5 may be written in the form:

$$
\frac{\partial}{\partial t}\left(\rho u_{i}\right)+\frac{\partial}{\partial x_{j}}\left(\rho u_{i} u_{j}\right)=\left(-\frac{\partial}{\partial x_{i}} p+\rho_{r e f} g_{i}\right)+\frac{\partial \tau_{i j}}{\partial x_{j}}+\left(\rho-\rho_{r e f}\right) g_{i}
$$

Applying the steps for Equations C-6 to C-9 on Equation C-13 yields:

$$
\begin{aligned}
& \frac{\partial}{\partial t^{*}}\left(\rho^{*} u_{i}^{*}\right)+\frac{\partial}{\partial x_{j}^{*}}\left(\rho^{*} u_{i}^{*} u_{j}^{*}\right) \\
& \quad=-\left(\frac{\partial p^{*}}{\partial x_{i}^{*}}+R i\right)+\frac{\partial}{\partial x_{j}^{*}} \tau_{i j}^{*}+\frac{x_{r e f}}{\rho_{r e f} u_{r e f}^{2}}\left(\rho-\rho_{r e f}\right) g_{i}
\end{aligned}
$$

Now, 


$$
\begin{aligned}
\left.\beta_{\text {ref }}=-\frac{1}{\rho_{\text {ref }}}\left(\frac{\partial \rho}{\partial T}\right]_{\mathrm{p}}\right)_{\text {ref }} & \approx-\frac{1}{\rho_{\text {ref }}}\left(\frac{\Delta \rho}{\Delta T}\right)_{\text {ref }} \approx-\frac{1}{\rho_{\text {ref }}} \frac{\rho-\rho_{\text {ref }}}{T-T_{\text {ref }}} \\
\frac{\rho-\rho_{\text {ref }}}{\rho_{\text {ref }}} & \approx-\beta_{\text {ref }}\left(T-T_{\text {ref }}\right)
\end{aligned}
$$

Let

$$
R i^{\prime}=\frac{g_{i} x_{r e f}}{u_{r e f}^{2}} \beta_{r e f}\left(T-T_{r e f}\right)
$$

Then Equation C-14 becomes:

$$
\frac{\partial}{\partial t^{*}}\left(\rho^{*} u_{i}^{*}\right)+\frac{\partial}{\partial x_{j}^{*}}\left(\rho^{*} u_{i}^{*} u_{j}^{*}\right)=-\left(\frac{\partial p^{*}}{\partial x_{i}^{*}}+R i\right)+\frac{\partial}{\partial x_{j}^{*}} \tau_{i j}^{*}+R i^{\prime}
$$

\section{C.3 Derivation of Non-Dimensional Energy Equation}

Conservation of thermal energy (conservation form)

$$
\frac{\partial}{\partial t}(\rho h)+\frac{\partial}{\partial x_{j}}\left(\rho u_{i} h\right)=\frac{D p}{d t}+\frac{\partial}{\partial x_{i}}\left(k \frac{\partial T}{\partial x_{i}}\right)+\frac{\partial}{\partial x_{j}}\left(u_{j} \frac{\partial \tau_{i j}}{\partial x_{j}}\right)
$$

If it is assumed that heat transfer occurs through a thermodynamically constant-pressure process and assumed that the contributions of viscous dissipation in the energy balance are negligible, then Equation C-18 becomes:

$$
\frac{\partial}{\partial t}(\rho h)+\frac{\partial}{\partial x_{j}}\left(\rho u_{i} h\right)=\frac{\partial}{\partial x_{i}}\left(k \frac{\partial T}{\partial x_{i}}\right)
$$

Multiply Equation C-19 by $x_{r e f} \rho_{r e f} u_{r e f}$ :

$$
\frac{\partial}{\partial t^{*}}\left(\rho^{*} h\right)+\frac{\partial}{\partial x_{i}^{*}}\left(\rho^{*} u_{i}^{*} h\right)=\frac{\partial}{\partial x_{i}^{*}}\left(\frac{k}{\rho_{\text {ref }} u_{r e f}} \frac{\partial T}{\partial x_{i}}\right)
$$

multiply Equation C-20 by $\beta_{r e f} / C_{p, r e f}$, and let: 


$$
h^{*}=\frac{h}{C_{p, r e f} / \beta_{r e f}}
$$

then,

$$
\begin{gathered}
\frac{\partial}{\partial t^{*}}\left(\rho^{*} h^{*}\right)+\frac{\partial}{\partial x_{i}^{*}}\left(\rho^{*} u_{i}^{*} h^{*}\right)=\frac{\partial}{\partial x_{i}^{*}}\left(\frac{k \beta_{r e f}}{C_{p, r e f} \rho_{r e f} u_{r e f}} \frac{\partial T}{\partial x_{i}}\right) \frac{x_{r e f}}{x_{r e f}} \\
\underbrace{\frac{\partial}{\partial t^{*}}\left(\rho^{*} h^{*}\right)+\frac{\partial}{\partial x_{i}^{*}}\left(\rho^{*} u_{i}^{*} h^{*}\right)}_{\frac{D\left(\rho^{*} h^{*}\right)}{D t^{*}}}=\frac{\partial}{\partial x_{i}^{*}}\left(\frac{k \beta_{r e f}}{C_{p, r e f} \rho_{r e f} u_{r e f}} \frac{\partial T}{\partial x_{i}^{*}}\right)
\end{gathered}
$$

given:

$$
\partial T=\frac{1}{C_{p}} \partial h, \quad \text { and } \quad \operatorname{Pr}=\frac{\mu_{r e f} C_{p, r e f}}{k_{r e f}}
$$

Noting Equations C-23 and C-11, Equation C-22(b) becomes:

$$
\begin{gathered}
\frac{D\left(\rho^{*} h^{*}\right)}{D t^{*}}=\frac{\partial}{\partial x_{i}^{*}}\left(\frac{k}{C_{p}} \frac{1}{\rho_{r e f} u_{r e f} x_{r e f}} \frac{\beta_{r e f}}{C_{p, r e f}} \frac{\partial h}{\partial x_{i}^{*}}\right)\left(\frac{\mu_{r e f}}{\mu_{r e f}} \frac{k_{r e f}}{k_{r e f}} \frac{C_{p, r e f}}{C_{p, r e f}}\right) \\
\frac{D\left(\rho^{*} h^{*}\right)}{D t^{*}}=\frac{\partial}{\partial x_{i}^{*}}\left(\frac{k^{*}}{C_{p}{ }^{*}} \frac{1}{R e} \frac{\beta_{r e f}}{C_{p, r e f}} \frac{\partial h}{\partial x_{i}^{*}}\right)\left(\frac{1}{P r}\right)
\end{gathered}
$$

here

$$
k^{*}=\frac{k}{k_{r e f}}, \quad \text { and } \quad C_{p}^{*}=\frac{C_{p}}{C_{p, r e f}}
$$

\section{C.4 Derivation of Non-Dimensional Heat Transfer Boundary Condition}

At the wall, the heat transfer rate is determined by the Fourier law of conduction:

$$
Q_{w}^{\prime \prime}=-\left.k_{w} \frac{\partial T}{\partial x}\right|_{w}
$$

Noting Equation C-23 


$$
\begin{gathered}
Q_{w}^{\prime \prime}=-\left.\frac{k_{w}}{C_{p, w}} \frac{\partial h}{\partial x}\right|_{w} \\
Q_{w}^{\prime \prime} \frac{C_{p, w}}{k_{w}}=-\left.\frac{\partial h}{\partial x}\right|_{w}
\end{gathered}
$$

multiply Equation C-27 by $x_{r e f} \beta_{r e f} / C_{p, r e f}$ :

$$
\begin{aligned}
& C_{p, w}^{*} \frac{Q_{w}^{\prime \prime} x_{r e f} \beta_{r e f}}{k_{w}}=-\left.\frac{\partial h^{*}}{\partial x^{*}}\right|_{w} \\
& \frac{Q_{w}^{\prime \prime} x_{r e f} \beta_{r e f}}{k_{w}}=-\left.\frac{1}{C_{p, w}^{*}} \frac{\partial h^{*}}{\partial x^{*}}\right|_{w}
\end{aligned}
$$

Equation C-28(b) is the form of the dimensional heat transfer rate used by Jackson, 2011. Alternative forms can be derived, for example, if

$$
h_{\text {Cheng }}^{*}=\frac{h}{C_{p, r e f} T_{r e f}}
$$

Then, multiply Equation C-29 by $x_{r e f} /\left(C_{p, r e f} T_{r e f}\right)$ to get the dimensional heat transfer rate used by Cheng et al. (2011):

$$
\frac{Q_{w}^{\prime \prime} x_{r e f}}{k_{w} T_{r e f}}=-\left.\frac{1}{C_{p, w}^{*}} \frac{\partial h^{*}}{\partial x^{*}}\right|_{w}
$$

To derive the version of the non-dimensional heat transfer rate by Ambrosini (2011), Equation $\mathrm{C}-28(\mathrm{~b})$ is divided by the Peclet number $(\mathrm{Pe})$ and multiplied by the ratio of the heat transfer area and the flow area:

$$
\begin{gathered}
\frac{Q_{w}^{\prime \prime} x_{r e f} \beta_{\text {ref }}}{k_{w}} \frac{1}{P e} \frac{A_{H T}}{A_{\text {Flow }}}=-\left.\frac{1}{C_{p, w}^{*}} \frac{\partial h^{*}}{\partial x^{*}}\right|_{w} \frac{1}{P e} \frac{A_{H T}}{A_{\text {Flow }}} \\
\frac{Q_{w}^{\prime \prime} x_{r e f} \beta_{\text {ref }}}{k_{w}} \frac{1}{P e} \frac{A_{H T}}{A_{\text {Flow }}}=\frac{Q_{w}^{\prime \prime} x_{\text {ref }} \beta_{\text {ref }}}{k_{w}} \frac{k_{\text {ref }}}{G_{\text {ref }} x_{\text {ref }} C_{p, \text { ref }}} \frac{A_{H T}}{A_{\text {Flow }}}
\end{gathered}
$$

If the wall thermal conductivity is proportional to the reference thermal conductivity then: 


$$
\frac{Q_{w} \beta_{r e f}}{\dot{m}_{r e f} C_{p, r e f}} \propto-\left.\frac{1}{C_{p, w}^{*}} \frac{\partial h^{*}}{\partial x^{*}}\right|_{w} \frac{1}{P e} \frac{A_{H T}}{A_{\text {Flow }}}
$$




\section{Appendix D Permissions to Reproduce Copyrighted Material}

Permissions for: Azih, C. Brinkerhoff, J.R. \& Yaras, M.I., 2012, 'Direct numerical simulation of convective heat transfer in a zero-pressure-gradient boundary-layer with supercritical water', Journal of Thermal Science, 21, pp.49-59

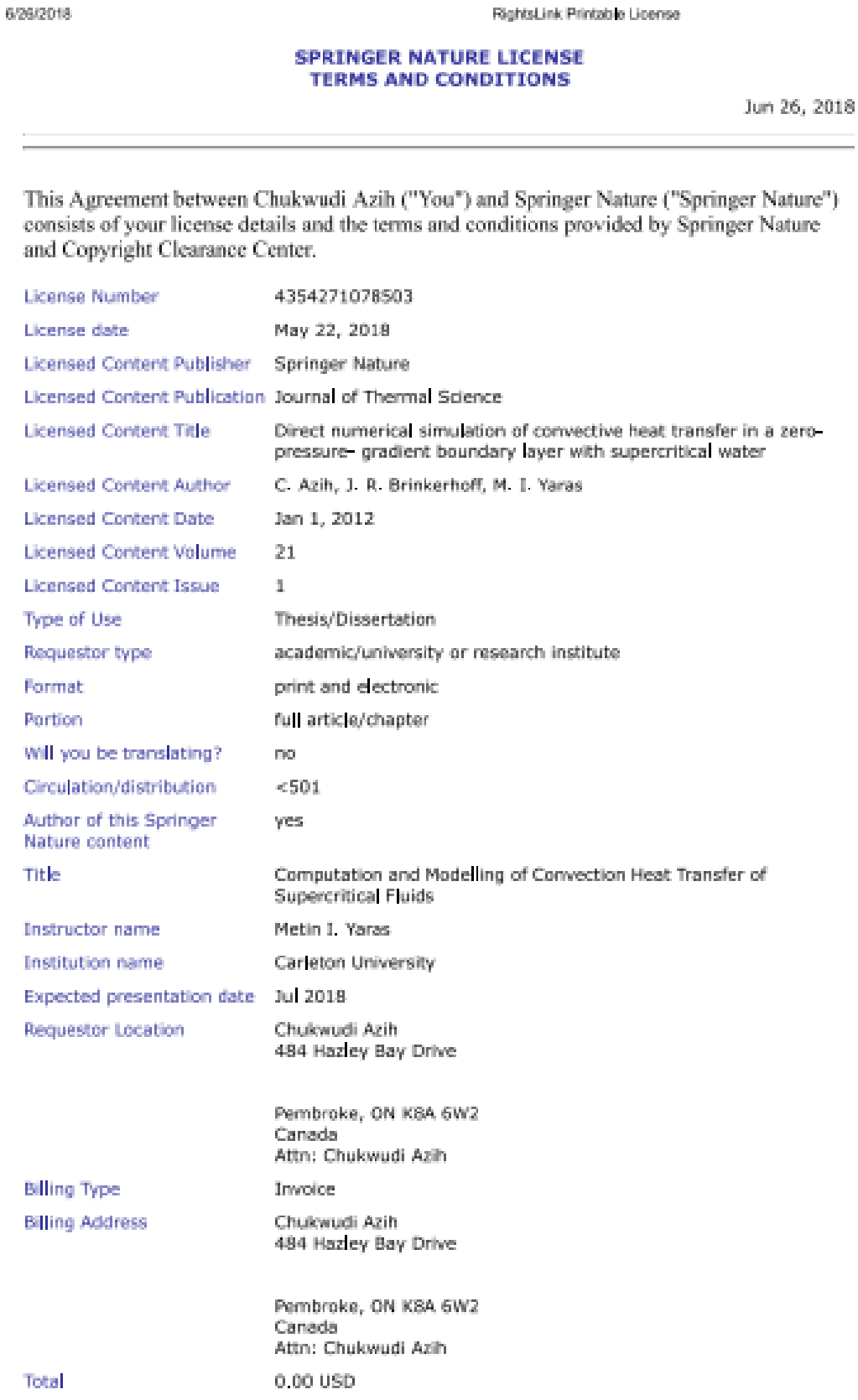


Permissions for: Azih, C. \& Yaras, M.I., 2017, 'Similarity criteria for modelling mixedconvection heat transfer in ducted flows of supercritical fluids', Journal of Heat Transfer, 122501, 139(12), 13 pages.

\title{
American Society of Mechanical Engineers ASME LICENSE
} TERMS AND CONDITIONS

Jun 26, 2018

\begin{abstract}
This is a License Agreement between Chukwudi Azih ("You") and American Society of Mechanical Engineers ASME ("American Society of Mechanical Engineers ASME") provided by Copyright Clearance Center $\left({ }^{\circ} \mathrm{OCC}\right)$. The license consists of your order details. the terms and conditions provided by American Society of Mechanical Engineers ASME, and the payment terms and conditions.
\end{abstract}

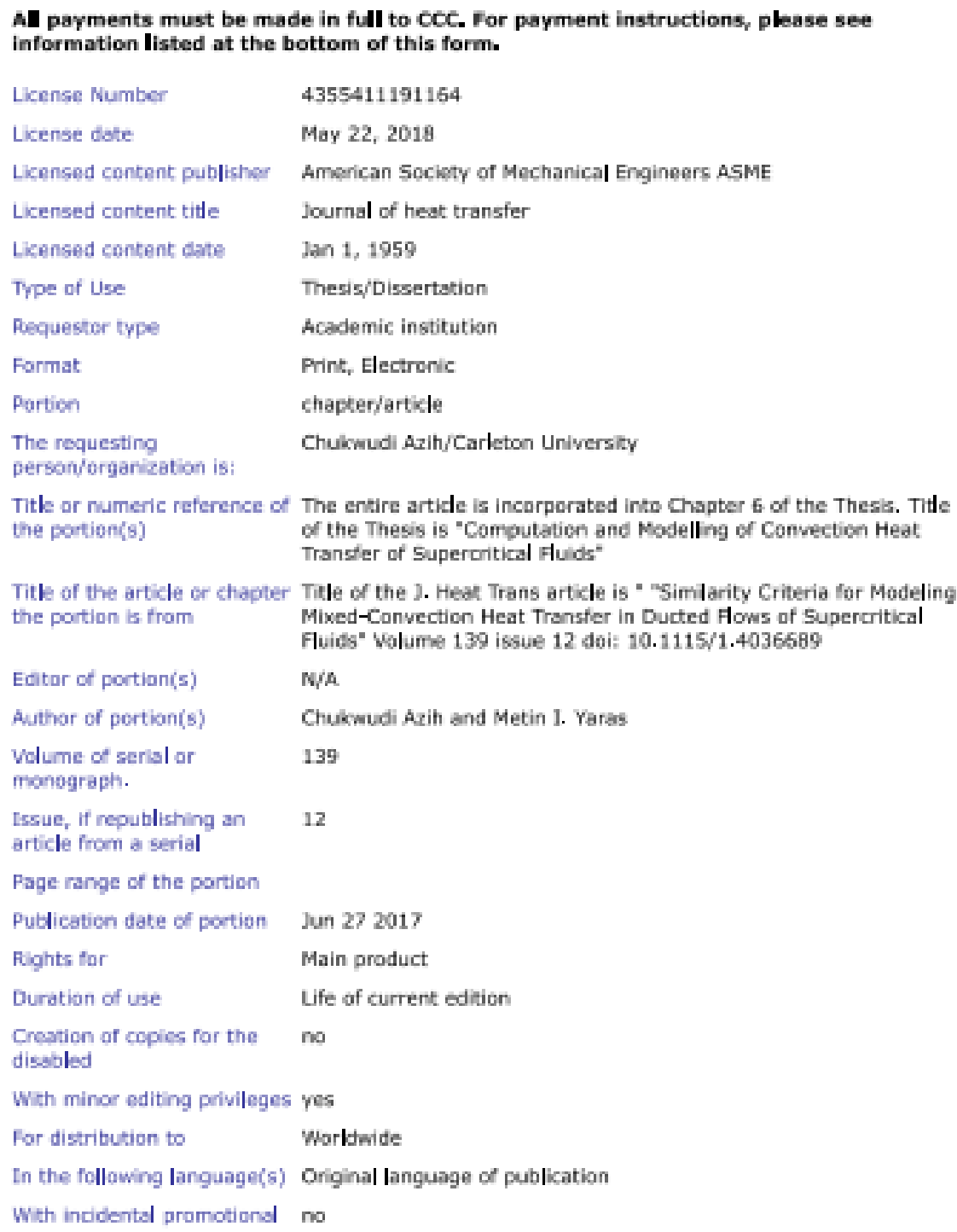




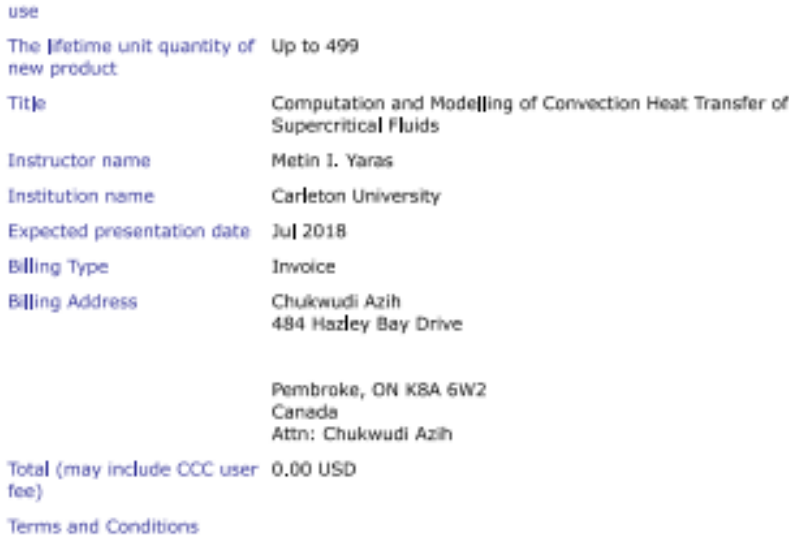

1. Deseription of Service; Defined Terms. This Republication License enables the User to obtain licenses for republication of one or more copyrighted works as described in detail on the relevant Order Confirmation (the "Work(s)"). Copyright Clearance Center, Inc. ("CCC") grants licenses through the Service on behalf of the rightsholder identified on the Onder Confimation (the "Rightsholder"). "Republication", as used herein, generally means the inclusion of a Work, in whole or in part, in a new work or works, also as described on the Order Confirmation. "User", as used herein, means the person or entity making such republication.

2. The terms set forth in the relevant Order Confirmation, and any terms set by the Rightsholder with respect to a particular Work, govern the terms of use of Works in connection with the Service. By using the Service, the person transacting for a republication license on behalf of the User represents and warrants that he'shefit (a) has been duly authorized by the User to accept, and hereby does acoept, all such terms and conditions on behalf of User, and (b) shall inform User of all such terms and conditions. In the event such person is a "freelancer" or other third party independent of User and $\mathrm{CCC}$, such party shall be deemed jointly a "User" for purposes of these terms and conditions. In any event, User shall be deemed to have accepted and agreed to all such terms and conditions if User repablishes the Work in any fashion.

3. Scope of License; Limitations and Obligations.

3.1 All Works and all rights therein, including copyright rights, remain the sole and exclusive property of the Rightsholder. The license created by the exchange of an Order Confirmation (and/or any inwoice) and payment by User of the full amount set forth on that document includes only those rights expressly set forth in the Order Confirmation and in these terms and conditions, and conveys no other rights in the Work(s) to User. All rights not expressly granted are hereby reserved.

3.2 General Payment Terms: You may pay by credit card or through an acoount with us payable at the end of the month. If you and we agree that you may establish a standing account with $\mathrm{CCC}$, then the following terms apply: Remit Payment to: Copyright Clearance 